Pontifícia Universidade Católica $_{\text {mato }}$

Raphael Chalhub Oliveira Spinelli Ribeiro

\title{
3D Visualization of Oil Displacement by a
}

Suspension of Microcapsules

Dissertação de Mestrado

Dissertation presented to the Programa de Pós-Graduação em Engenharia Mecânica of PUC-Rio in partial fulfillment of the requirements for the degree of Mestre em Engenharia Mecânica.

Advisor: Prof. Márcio da Silveira Carvalho 


\title{
Raphael Chalhub Oliveira Spinelli Ribeiro
}

\section{D Visualization of Oil Displacement by a Suspension of Microcapsules}

\begin{abstract}
Dissertation presented to the Programa de Pós-Graduação em Engenharia Mecânica of PUC-Rio in partial fulfillment of the requirements for the degree of Mestre em Engenharia Mecânica. Approved by the Examination Committee.
\end{abstract}

Prof. Márcio da Silveira Carvalho

Advisor

Departamento de Engenharia Mecânica - PUC-Rio

Prof. Marcos Vitor Barbosa Machado

Petróleo Brasileiro S.A. - Petrobras

Prof. Luís Fernando Alzuguir Azevedo

Departamento de Engenharia Mecânica - PUC-Rio 
All rights reserved.

\section{Raphael Chalhub Oliveira Spinelli Ribeiro}

Bachelor in mechanical engineering by Pontifícia Universidade

Católica do Rio de Janeiro (2016)

Bibliographic data

Chalhub Ribeiro, Raphael

3D Visualization of Oil Displacement by a Suspension of Microcapsules / Raphael Chalhub Oliveira Spinelli Ribeiro; advisor: Márcio da Silveira Carvalho. - Rio de janeiro: PUCRio, Departamento de Engenharia Mecânica, 2019.

v., 150 f: il. color. ; $30 \mathrm{~cm}$

Dissertação (mestrado) - Pontifícia Universidade Católica do Rio de Janeiro, Departamento de Engenharia Mecânica.

Inclui bibliografia

1. Engenharia Mecânica - Teses. 2. Microscopia confocal:. 3. Recuperação de óleo:. 4. Microcápsulas:. 5. Microfluídica. I. da Silveira Carvalho, Márcio. II. Pontifícia Universidade Católica do Rio de Janeiro. Departamento de Engenharia Mecânica. III. Título.

CDD: 620.1 
To my friends and family, for their love and support. 


\section{Acknowledgments}

First, I would like to demonstrate my gratitude for my parents, Tânia and Geraldo, and thank them for being present in my whole life as good friends. I have learned a lot from them, it was not hard since they are excellent people and very good teachers. I would like to thank also Josefa for being a helpful person, caring about me and my family since I was a playful kid. I appreciate their support that made my experience of Master's studies less stressful every day.

To my advisor, Professor Marcio Carvalho, for the opportunity to work in his research group, the experience of participating in his classes and for the patience on discussing and analyzing the achievements obtained on each step of this study.

To Professor Sidnei Paciornik for his attention during our meetings in which we long discussed image processing methods and for the great IPDI lessons that were of extreme importance to develop this work.

To Débora Nascimento and José Vimieiro Junior for the dedication and expertise obtained working on the confocal microscopy technique towards oil recovery visualization.

To my friends João Lucas and João Pedro Mello for all trips to Araras when it was necessary to relax from the studies and sail lessons and for the time we spent on bars, playing cards and chatting about life.

To Perola Barbosa, Isabel Oberg, Julia Besserman and Laura Ribeiro for the friendship since Escola Parque. For the pizzas and the good time we had almost every week. To Barbara Furst and João Felippe for, in addition to the almost weekly meetings, for their friendship and game nights.

To Gabriel Caju, who has been my friend longer than anyone else, for the sail partnership started when we had only 9 years old in which we grew together. The championships and sailing trips were very important in building the person I am today. Thanks also for the time and patience dedicated to when we developed the sailing school.

To Yuri Martins for the time dedicated teaching me to sail a Snipe, class of boat that gave me a new perspective on what is the sailing sport. Besides sailing, I would like to thank him also for cycling and triathlon motivation.

To Luis Paulo, a great sailor and sailing coach with whom I agreed to pass hours together training to improve our sailing abilities. The time on each car trip pulling our boat for championships was very important, as well as the achievements we got. 
To all colleagues I shared classes through graduation and masters, but especially to José Eduardo for being a good friend, sharing difficulties and being a great partner working on class' assignments.

To all my friends from the LMMP group, in particular, Mariano, Paula, Marisa, Thiago, Sérgio, Bruna, Frederico, Ranena, Paulo, Danmer, Tálita, Débora, Jorge, José, Monique, Lisbeth, Gabriel, Daniele and Marcelo for the good times and knowledge we shared. To Felicle, Leonardo and Bianca for all the help they gave me on the laboratory and the company when preparing the experiment.

To Asafe Bittencourt from the Laboratory of Metallography and Heat Treatment (LMTT) for letting me use the furnace from their facility and being always helpful.

To the National Council of Scientific and Technological Development $(\mathrm{CNPq})$ for the scholarship on my studies that made this work and all results possible and also for helping the development of researches in many different areas.

Finally, this work was also the result of all previous relations I had before entering the masters that contributed directly or indirectly to my personal development and I am grateful to everyone. 


\section{Abstract}

Chalhub Ribeiro, Raphael; da Silveira Carvalho, Márcio (Advisor). 3D Visualization of Oil Displacement by a Suspension of Microcapsules. Rio de Janeiro, 2019. 150p. Dissertação de Mestrado - Departamento de Engenharia Mecânica, Pontifícia Universidade Católica do Rio de Janeiro.

Thanks to decay of new discoveries of oil and gas reserves in the past decades, oil companies have a growing interest in the increase of oil recovery efficiency. Commonly, after primary and secondary recovery phases, a large amount of oil remains inside the reservoir, as it becomes unprofitable to continue the extraction. Thus, the number of studies focused on enhanced oil recovery is growing, aiming to obtain a better recovery fraction. The focus of this work is to study the fundamentals of oil displacement in porous media using a confocal laser scanning microscope, which enables 3D visualization with a good resolution. The analysis was on oil displacement that results from the use of a suspension of gellan gum microcapsules in water injected after water injection. These microcapsules, moving along with the water, blocked some of the preferential paths and forced the water to displaces parcels of the trapped oil. The result achieved was a collection of 3D images from artificial porous media, in which it was possible to distinguish the distribution of phases (microcapsules, oil, and aqueous phases) inside the porous media, before and after the microcapsules injection. These images showed that indeed the gellan gum microcapsules blocked preferential water paths and that, after the blockage, some oil ganglia were displaced from their original positions. This thesis applies modern techniques of microscopy to investigate the concept behind enhanced oil recovery using microcapsules.

\section{Keywords}

Confocal microscopy: Oil recovery: Microcapsules: Microfluidics. 


\section{Resumo}

Chalhub Ribeiro, Raphael; da Silveira Carvalho, Márcio. Visualização 3D do Deslocamento de Óleo por uma Suspensão de Microcápsulas. Rio de Janeiro, 2019. 150p. Dissertação de Mestrado - Departamento de Engenharia Mecânica, Pontifícia Universidade Católica do Rio de Janeiro.

Devido à diminuição do número de descobertas de novas reservas de óleo e gás nas últimas décadas, as companhias de petróleo têm demonstrado um interesse cada vez maior em melhorar a eficiência dos processos de recuperação de óleo. Geralmente, após as fases de recuperação primário e secundário, uma grande quantidade de óleo permanece dentro do reservatório, pois a extração se torna não rentável. Assim, cresce o número de estudos voltados para a recuperação avançada de petróleo, com o objetivo de obter uma melhor fração de recuperação. O foco deste trabalho é estudar os fundamentos do deslocamento de óleo em meios porosos usando um microscópio confocal de varredura a laser, que possibilita visualizações $3 \mathrm{D}$ com boa resolução. A análise foi no deslocamento de óleo resultante da injeção de uma suspensão de capsulas de goma gelana em água após a injeção de água. Estas capsulas, movendo com a água, bloqueiam alguns dos caminhos preferenciais e forçam a água a deslocar uma parte do óleo preso. O resultado alcançado foi uma coleção de imagens 3D de meios porosos artificiais, nas quais foi possível distinguir a distribuição das fases (microcápsulas, fase aquosa e oleosa) dentro dos meios porosos, antes e após a injeção das microcápsulas. Essas imagens mostraram que as microcápsulas de goma gelana bloqueiam os caminhos preferenciais da água e que, após o bloqueio, alguns gânglios de óleo foram deslocados de suas posições originais. Esta tese aplica técnicas modernas de microscopia para examinar o conceito por trás da recuperação avançada de óleo usando microcápsulas.

\section{Palavras-chave}

Microscopia confocal: Recuperação de óleo: Microcápsulas: Microfluídica. 


\section{Table of contents}

1 Introduction $\quad 23$

1.1 Motivation 23

1.2 Dissertation goals 25

1.3 Scope of work 26

2 Theoretical Framework and Literature Review 27

2.1 Flow in porous media 27

2.2 Oil recovery 33

2.2.1 Primary and secondary oil recovery methods 34

2.2.2 Enhanced oil recovery 41

2.3 Microcapsules as a recovery method 49

2.4 Confocal laser scanning microscopy $\quad 57$

2.4.1 CLSM technique applied to porous media flow 60

2.5 Image processing 66

3 Experimental Procedure $\quad 69$

3.1 General description of the experiments 69

$\begin{array}{ll}3.1 .1 & \text { Device fabrication }\end{array}$

$\begin{array}{lll}3.1 .2 & \text { Device holder } & 76\end{array}$

$\begin{array}{ll}3.1 .3 \text { Fluid choice } & 77\end{array}$

$\begin{array}{lll}\text { 3.1.3.1 Refractive index } & 79\end{array}$

$\begin{array}{lll}\text { 3.1.3.2 Fluorescent pigments } & 81\end{array}$

3.1.4 Microcapsules $\quad 85$

$\begin{array}{ll}3.2 \text { Procedure } & 87\end{array}$

3.2.1 Device saturation with oil $\quad 88$

$\begin{array}{ll}3.2 .2 & \text { Injections procedure }\end{array}$

$\begin{array}{ll}\text { 3.2.3 Microscope settings } & 91\end{array}$

$\begin{array}{ll}\text { 3.3 Data processing } & 96\end{array}$

$\begin{array}{lll}\text { 3.3.1 } & \text { Pre-processing } & 97\end{array}$

$\begin{array}{lll}\text { 3.3.2 Segmentation } & 98\end{array}$

$\begin{array}{lll}\text { 3.3.3 Post-processing } & 99\end{array}$

$\begin{array}{ll}\text { 3.3.4 Data acquisition for volume quantification } & 99\end{array}$

$4 \quad$ Results and discussions $\quad 101$

4.1 Visual qualitative results with homogeneous porous media 101

4.1.1 Preliminary results using devices " $d$ " and "i" 101

4.1.2 Results using devices "I" and "II" 111

$\begin{array}{lll}\text { 4.1.2.1 } & \text { First experiment } & 113\end{array}$

$\begin{array}{ll}\text { 4.1.2.2 Second experiment } & 114\end{array}$

$\begin{array}{ll}\text { 4.1.2.3 Third experiment } & 117\end{array}$

4.1.2.4 Fourth experiment 118

4.1.2.5 Fifth experiment 119

4.2 Visual qualitative results with stratified porous media 126

$\begin{array}{ll}\text { 4.2.1 Sixth experiment } & 127\end{array}$ 
$\begin{array}{lll}\text { 4.2.2 Seventh experiment } & 130\end{array}$

$\begin{array}{ll}4.3 & 132\end{array}$

$\begin{array}{lll}4.4 & \text { Dynamic visualization } & 136\end{array}$

$\begin{array}{lll}\text { 4.5 Capillary devices with thinner walls } & 138\end{array}$

5 Conclusions $\quad 140$

5.1 Remarks on the experimental setup 140

5.1.1 Remarks on the technique for visualization with the CLSM 140

5.1.2 Remarks on the experimental setup for microcapsules injection in porous media 141

5.2 Remarks on the results obtained 141

$\begin{array}{lll}5.3 & \text { Future works } & 143\end{array}$

5.4 Final remarks 144

$\begin{array}{lr}\text { Bibliography } & 145\end{array}$

$\begin{array}{ll}\text { Bibliography } & 145\end{array}$

$\begin{array}{ll}\text { A Published paper } & 150\end{array}$ 


\section{List of figures}

Figure 1.1 Emusions blocking the preferential path. Adapted from Engelke [1].

Figure 2.1 Diagram of a flow through a porous media.

Figure 2.2 Illustration of the forces between molecules in the interface of two different materials.

Figure 2.3 Illustration of wetting and non-wetting fluid configuration. 31

Figure 2.4 Illustration of the capillary effect on water wetting the cylindrical glass tube.

Figure 2.5 Non wetting fluid flow through a porous channel. Image by Lenormand [2].

Figure 2.6 Typical relative permeability curve.

Figure 2.7 Image adapted from Chuoke et al. [3]. Two examples of water displacing oil and forming the viscous fingering pattern. (a) $\mu_{o}=9.45 \mathrm{cP}, K=96 D, \mu_{w}=1 \mathrm{cP}, U=0.00932 \mathrm{~cm} / \mathrm{s}$ and $\sigma=42$ dyne $/ \mathrm{cm}$; (b) $\mu_{o}=66 \mathrm{cP}, k=65 \mathrm{D}, \mu_{w}=0.936 \mathrm{cP}$, $U=0.0106 \mathrm{~cm} / \mathrm{s}$ and $\sigma=48$ dyne $/ \mathrm{cm}$.

Figure 2.8 Adapted from Kantzas et al. [4]. Illustration of viscous fingering effect on the injection of water in a porous medium. The preferential path is formed and, even in the area that the water sweep the oil, some ganglia is left by the capillary pressure. 38

Figure 2.9 Normalized residual oil saturation measured using 3D confocal micrographs. Image from Datta et al. [5].

Figure 2.10 Illustration by Payatakes [6]. Oil ganglion behavior at (a) no flow; (b) aqueous phase flow from left to right, pressure gradient does not reach critical value; (c) the pressure gradient is higher than the critical value, ganglion was mobilized.

Figure 2.11 Images from Hinkley's [7] movie films. Movement of a ganglion at a porous medium, composed by glass beads, and different moments of the fission process.

Figure 2.12 Diagram from Olajire [8] of some EOR processes.

Figure 2.13 Result from McAuliffe [9], the difference on fluid permeability after the injection of emulsion with two different droplet sizes.

Figure 2.14 From McAuliffe [9], a schematic diagram of the experimental set up to test cores of different permeabilities mounted in parallel.

Figure 2.15 From Cobos [10]. Evolution of the inlet pressure at $\mathrm{Q}=$ $0.04 \mathrm{ml} / \mathrm{h}$ of a larger emulsion and images of the flow near the capillary throat at the instants highlighted in the plot.

Figure 2.16 Adapted from McAuliffe [9]. Map of the Midway-Sunset field and well location.

Figure 2.17 From McAuliffe [11]. On top, the fluid production from the waterflood area. At the bottom, the fluid production on the emulsion treated area. 
Figure 2.18 Double step emulsion producing. In blue is represented the aqueous phase, in yellow and orange are represented the inner and outer oily phases, respectively.

Figure 2.19 Illustration from Nascimento et al. [12]. Sketch of the microdevice for the capsule production.

Figure 2.20 Result of the capsules elasticity from Nascimento et al. [12]. Plot of apparent modulus (maximum stress to maximum strain ratio) as function of shell thickness. Each color represent a different polymer to cross-link ratio.

Figure 2.21 Adapted from Vimieiro [13]. Result of the gradual increase in water injection observed on one 2D slice of the porous core. Green in the first image and white in the others represents the aqueous phase. From left to right images show first the whole section of the core prior to image processing. Then, a fixed section of the core is shown: saturated with the aqueous phase; saturated with the oil phase; and after the aqueous phase injection at $21.355 \mu \mathrm{l} / \mathrm{h}, 106.755 \mu \mathrm{l} / \mathrm{h}, 533.775 \mu \mathrm{l} / \mathrm{h}, 2.67 \mathrm{ml} / \mathrm{h}$ and $13.35 \mathrm{ml} / \mathrm{h}$, respectively. Fluid inlet is at the bottom side of the core.

Figure 2.22 Subtraction operation done by Vimieiro [13] to discover the amount of remaining oil in each image. "I" is the section saturated with water, "II" is the section after running the desired flow rate and "III" is the subtraction "I"-"II". In "I" and "II" water is presented on white and the oil and the porous core in black. In "III", oil is in white and water and the porous core are presented in black.

Figure 2.23 Oil residual saturation for different devices as a function of the Capillary Number, from Vimieiro [13].

Figure 2.24 Adapted from Michelon et al. [14]. (a) shows a perspective view from a model of the glass capillary device; (b) shows a diagram of the region of droplet formation. $Q_{i}, Q_{m}$ and $Q_{o}$ are the flow rates of inner, middle and continuous phases, respectively; (c) shows a picture of capsules being formed; and (d) informs the geometric parameters of the devices used. $d_{i}$ and $d_{c}$ are the inner diameter of the injection and collection capillary tubes, respectively, and $l$ denotes the separation distance between them.

Figure 2.25 Image from Semwogerere and Weeks [15]. Images of cells of spirogyra generated by (A) normal microscope and (B) confocal microscope .

Figure 2.26 Different focal planes of a pollen grain visualized on the confocal microscope are shown numbered on the left side and a $3 \mathrm{D}$ reconstruction many thin slices is presented on the right side of the figure. Adapted from Claxton et al. [16]. 
Figure 2.27 Illustration of the concept of the pinhole tool from Borlinghaus [17]. The laser (in green) excites the sample not only in the focal plane, but also above and below. Light emitted from above and below the focal plane will not pass the pinhole. Only light that originates from the focal plane will reach the detector.

Figure 2.28 Illustration of fluorescence emission from Semwogerere and Weeks [15]. Molecules absorbs photon energy up to a higher energy state, loses a little of that energy to other molecules and then emit the rest emitting light of a lower energy.

Figure 2.29 Images obtained by Hovis and Heuer [18]. (a) Conventional bright field image of a martensitic stainless steel sample. (b) An image of the same region taken using CLSM technique.

Figure 2.30 Experiment performed by Krummel et al. [19]. Pictures on the left show the porous medium saturated with aqueous (wetting) phase and an oil ganglion. In the following pictures, showing the time evolution of the drainage, numerical image processing was performed so to show only the invading phase. The drainage efficiency clearly depends on $\mathrm{Ca}$.

Figure 2.31 Imbibition experiment performed by Krummel et al. [19]. Sequences show the aqueous (wetting) phase displacing oil along the time. Imbibition efficiency highly depends on $\mathrm{Ca}$.

Figure 2.32 3D images showing ganglia left in the porous device after imbibition at different values for $C a$. Image from Krummel et al. [19].

Figure 2.33 Schematic view of one of the stratified devices, showing a clear division between the coarse and fine strata. Image from Datta and Weitz [20].

Figure 2.34 Drainage by oil in a stratified porous medium for increasing values of $C a$. Image adapted from Datta and Weitz [20].

Figure 2.35 Image from Datta et al. [5], showing the porous device (a); the imbibition front invading from left to right (b); and one slice of a region in the porous medium in different times along the imbibition process.

Figure 2.36 Classical diagram of image processing.

Figure 3.1 Simplified diagram of the experiment.

Figure 3.2 Schematic illustration of the porous device.

Figure 3.3 Microscope image of a porous media with glass beads of $425 \mu \mathrm{m}$ diameter (left) and a stratified (two-layered) porous media with 250 and $425 \mu \mathrm{m}$ diameter glass beads (right).

Figure 3.4 Model of the firebrick used to hold the porous device vertically on the furnace.

Figure 3.5 Device cooling before the second sintering process for the stratified porous device.

Figure 3.6 Needles used in the laboratory for syringe-tube connections and their parts removed to manufacture the porous media device connection. 
Figure 3.7 3D view from the device holder and its measures.

Figure 3.8 Difference between previous porous devices used by Vimieiro [13] (a) from the one developed on this work (b). $\quad 76$

$\begin{array}{lll}\text { Figure 3.9 Device holder placed on the microscope stage. } & 77\end{array}$

Figure 3.10 Adaptation on positioning the porous device in the holder. 77

Figure 3.11 Oil and aqueous phase distinguished on the miscibility test. Aqueous phase is dyed light blue and the oil phase is dyed in yellow.

Figure 3.12 Method used by Vimieiro [13] to match the solids and fluids refractive indices.

Figure 3.13 Diagram of how the method of Yue et al. [21] can determine the refractive index of the microsphere (a). Microsphere image obtained by phase contrast microscope (b). Images of a polystyrene microsphere immerserd in water (c), ethanol (d), and a 1:1 mixture of glycerol and water (e).

Figure 3.14 Measurement of the refractive index of glass beads by the method proposed by Yue et al. [21].

Figure 3.15 Fluorescence leak detection kit, from Spectroline, used to perform excitation/emission tests the distinction on the oil phase and microcapsules.

Figure 3.16 Emission spectra from different fluorescent pigments after $488 \mathrm{~nm}, 552 \mathrm{~nm}$ and $638 \mathrm{~nm}$ wavelengths laser excitation.

Figure 3.17 Spectra and range of detection for each fluorescent pigment chosen. Separated and combined visualization of the oil phase (yellow) and the microcapsules (purple).

Figure 3.18 Microcapsules fabrication setup with three syringe pumps, an inverted microscope, a high-speed camera and a computer.

Figure 3.19 Diagram explaining the procedure for oil saturation of the porous device. The line filled with oil phase is shown in yellow and the line with aqueous phase is shown in blue.

Figure 3.20 Diagram of the startup of aqueous phase injection in the porous device. Oil is being recovered from the device. The line filled with oil phase is shown in yellow and the line with aqueous phase is shown in blue.

Figure 3.21 Diagram of aqueous phase injection in the porous device after steady state was established. Only aqueous phase is being recovered from the device. The line filled with oil phase is shown in yellow and the line with aqueous phase is shown in blue.

Figure 3.22 Diagram of aqueous phase with capsules injection on the porous medium. Additional oil phase was recovered from the device. The line filled with oil phase is shown in yellow and the line with aqueous phase is shown in blue.

Figure 3.23 Image formation procedure for the confocal laser scanning microscopy technique. The aqueous phase is shown in blue, the oil phase in yellow and the microcapsules in purple.

Figure 3.24 Software settings for the image acquisition. 
Figure 3.25 Selection of the range of acquisition for each detector (PMT and HyD) in the first sequential step. The oil phase was acquired using the PMT detector and the aqueous phase using the $\mathrm{HyD}$ detector.

Figure 3.26 Example of images from the aqueous phase detector channel, oil phase detector channel and microcapsules detector channel. The aqueous phase detected in the first image has lower intensity in the region occupied by the oil phase and microcapsules. The oil phase detects the fluorescence from the oil phase and from the microcapsules. The last image is from the microcapsules channel, in which only the microcapsules are detected.

Figure 3.27 Linear z-Compensation setting window for the first sequential step.

Figure 3.28 Macro file developed in ImageJ.

Figure 3.29 Residual oil inside the device after the injection of the aqueous phase. Comparison from the Otsu and Yen method for images with low pixel intensities and small amount of information. 98

Figure 3.30 ImageJ window to apply the configurations for the open operation.

Figure 4.1 3D perspective view of the middle region, as shown in the sketch, of the device "d" saturated with aqueous phase. Reconstruction formed by the stack of 210 images.

Figure 4.2 Example of 2D image used in the stack to recreate the $3 \mathrm{D}$ visualization shown in Fig. 4.1. The dark circles are the glass spheres that don't emit fluorescence and the aqueous phase is represented in blue.

Figure 4.3 3D perspective view of the device " $\mathrm{d}$ " after the oil phase saturation. The oil phase is shown in yellow and the aqueous phase in blue.

Figure 4.4 3D perspective view of the device "d" after the oil phase saturation showing (after software manipulation) only the remaining aqueous phase left inside the porous medium, called irreducible aqueous phase.

Figure 4.5 Comparison of the oil saturation at different flow rates. The upper image shows the result of a single slice of the 3D image at $100 \mu l / h$ and the bottom image shows the same plane after the increase of the oil phase flow rate to $500 \mu l / h$.

Figure 4.6 Detailed view of a single plane of the device "d" after the oil saturation. It is possible to observe the irreducible aqueous wetting phase in blue and the oil, non wetting phase, in yellow. The visualization area of the image is $581.25 \times 582.25 \mu \mathrm{m}$.

Figure 4.7 3D perspective view at the middle region of device "d" after the aqueous phase injection at $250 \mu l / h$. The aqueous phase displaced a large part of the oil phase. 
Figure $4.83 \mathrm{D}$ perspective view at the middle region of device "d" after large microcapsules injection at $250 \mu l / h$. The microcapsules tested in this experiment were not effective to increase the displacement of oil phase. In fact, it was not possible to observe any microcapsules in this region of the device.

Figure 4.9 Comparison between 2D images at the same z-position into the device "d" before and after the large microcapsules injection. It is possible to notice that the oil ganglia that appear in this plane didn't moved after the microcapsules injection.

Figure 4.10 Detailed view of a single tile, $581.25 \times 582.25 \mu \mathrm{m}, 2 \mathrm{D}$ image positioned at the entrance of the porous medium. The aqueous phase is represented in blue and the microcapsules are shown in purple. The aqueous phase flow rate was not enough to force the microcapsules inside the porous medium.

Figure 4.11 Images of the same plane after gradual increases of aqueous phase flow rate injection.

Figure 4.12 3D perspective view of the middle region of the device "i" saturated with aqueous phase. Reconstruction formed by the stack of 212 images.

Figure 4.13 3D perspective view of the middle region of the device "i" saturated with oil phase. Reconstruction formed by the stack of 212 images.

Figure 4.14 Detailed view of the device "i" saturated with oil phase displaying only the aqueous phase.

Figure $4.153 \mathrm{D}$ perspective view of the middle region of the device "i" after the aqueous phase injection. Reconstruction formed by the stack of 212 images.

Figure 4.16 3D perspective view of the middle region of the device "i" after the microcapsules injection. Reconstruction formed by the stack of 212 images.

Figure 4.17 3D image of device "i", formed by the stack of 212 images, viewed from bellow.

Figure 4.18 3D image of device "i", formed by the stack of 212 images, viewed from bellow without the display of the aqueous phase.

Figure 4.19 Oil leaving the porous device in the exit tube.

Figure 4.20 Bottom view from the middle section of the porous medium, as shown in the sketch. In the left is the image obtained after the injection of aqueous phase and in the right is the image obtained after the injection of aqueous phase with the microcapsules. The aqueous phase was hidden to facilitate the analysis.

Figure 4.21 Sketch showing the region where image were acquired after the second experiment.

Figure 4.22 Images of the region investigated with and without showing the aqueous phase. To mark the boundary of the porous medium, a red line was drawn in the image hiding the aqueous phase. 
Figure 4.23 Bottom view from side "A" of device "II" in the second experiment. In the left is the image obtained after the injection of aqueous phase and in the right is the image obtained after the injection of aqueous phase with the microcapsules. The aqueous phase was hidden to facilitate the analysis. A red line was drawn to mark the boundary of the porous medium.

Figure 4.24 Perspective view before and after the microcapsules injection in the second experiment. Visualization from side "A" with the aqueous phase hidden.

Figure 4.25 Detail of a oil ganglion that was not mobilized after the microcapsule blocking some pores near its position.

Figure 4.26 Bottom view from side "B" of device "II" in the second experiment. In the left is the image obtained after the injection of aqueous phase and in the right is the image obtained after the injection of aqueous phase with the microcapsules. The aqueous phase was hidden to facilitate the analysis. A red line was drawn to mark the boundary of the porous medium.

Figure 4.27 Bottom view from side "A" of device "I" in the third experiment. In the left is the image obtained after the injection of aqueous phase and in the right is the image obtained after the injection of aqueous phase with the microcapsules. The aqueous phase was hidden to facilitate the analysis. A red line was drawn to mark the boundary of the porous medium.

Figure 4.28 Bottom view from side "A" of device "I" in the fourth experiment. In the left is the image obtained after the injection of aqueous phase and in the right is the image obtained after the injection of aqueous phase with the microcapsules. The aqueous phase was hidden to facilitate the analysis. A red line was drawn to mark the boundary of the porous medium.

Figure 4.29 Bottom view from side "A" of device "I" in the fifth experiment. In the left is the image obtained after the injection of aqueous phase and in the right is the image obtained after the injection of aqueous phase with the microcapsules. The aqueous phase was hidden to facilitate the analysis. A red line was drawn to mark the boundary of the porous medium.

Figure 4.30 3D perspective view built by stacking 228 2D slices captured from side "A" before and after the microcapsules injection. The aqueous phase was hidden to facilitate the analysis. Notice the microcapsules located further downstream in the porous medium.

Figure 4.31 Bottom view from side "B" of device "I" in the fifth experiment. In the left is the image obtained after the injection of aqueous phase and in the right is the image obtained after the injection of aqueous phase with the microcapsules. The aqueous phase was hidden to facilitate the analysis. A red line was drawn to mark the boundary of the porous medium. 
Figure 4.32 3D perspective view built by stacking 228 2D slices captured from side "B" before and after the microcapsules injection. The aqueous phase was hidden to facilitate the analysis. Notice the presence of only one large ganglion at the inlet of the device before the porous medium.

Figure 4.33 Observation of the full depth of the porous medium by combining the 3D images acquired from opposite sides.

Figure 4.34 View from side "B" of a single 2D slice from device "I", at stack position 17, before and after the microcapsules injection for comparison.

Figure 4.35 Detailed view from a single plane at the entrance of the device "I". The upper image is the plane before the microcapsules injection and the bottom image is the result after the microcapsules injection.

Figure 4.36 Image acquired without the fluorescence detector showing the plane of acquisition, according to the sketch, in which both coarse and fine glass spheres are observed.

Figure 4.37 View from side "A" of the transversal plane of the device "VI" in the sixth experiment, before and after microcapsules injection. On the left of the image are the coarse spheres region and on the right are the fine spheres. The aqueous phase was hidden to facilitate the analysis. A red line was drawn to mark the boundary of the porous medium.

Figure 4.38 View from side "B" of the transversal plane of the device "VI" in the sixth experiment, before and after microcapsules injection. On the right of the image are the coarse spheres region and on the left are the fine spheres. The aqueous phase was hidden to facilitate the analysis. A red line was drawn to mark the boundary of the porous medium.

Figure 4.39 View from side of the coarse spheres of the device "V" in the seventh experiment, as shown in the sketch. In the left is the image obtained after the injection of aqueous phase and in the right is the image obtained after the injection of aqueous phase with the microcapsules. The aqueous phase was hidden to facilitate the analysis. A red line was drawn to mark the boundary of the porous medium.

Figure $4.403 \mathrm{D}$ perspective view built by stacking $2282 \mathrm{D}$ slices captured from the side of the coarse spheres before and after the microcapsules injection. The aqueous phase was hidden to facilitate the analysis.

Figure 4.41 View from side of the fine spheres of the device "V" in the seventh experiment, as shown in the sketch. In the left is the image obtained after the injection of aqueous phase and in the right is the image obtained after the injection of aqueous phase with the microcapsules. The aqueous phase was hidden to facilitate the analysis. A red line was drawn to mark the boundary of the porous medium. 
Figure 4.42 Perspective view of device "d" before and after the microcapsules injection.

Figure 4.43 Comparison graph between the $S_{\text {or }}$ values obtained using the Otsu automatic segmentation method (blue) and the Yen method (orange) for images from each step of the experiment with device "d".

Figure 4.44 Microscope settings for the dynamic visualization of capsules blocking pores. Aqueous phase is represented by blue and the microcapsules by yellow.

Figure 4.45 Different moments of the dynamic visualization.

Figure 4.46 Comparison between images obtained after aqueous phase saturation using devices with two different wall thickness. 139 


\section{List of tables}

Table 2.1 Table obtained by Sahimi [22]. Porosity range of different types of porous media.

Table 2.2 Injection of emulsion through cores of different permeabilities mounted in parallel. By McAuliffe [9].

Table 3.1 Porosity range of the devices developed in this work and the ones presented in the literature.

Table 3.2 Permeability from the devices developed in this work compared from the devices used by Carman [23] and Data [20]

Table 3.3 List of microcapsules produced using the Device \#2 and the fluorescent pigment mixed in the inner phase of the capsules. 86

Table 4.1 Quantification of the oil phase, aqueous phase and the residual oil saturation $\left(S_{\text {or }}\right)$ after each phase saturation and after the aqueous phase injection and aqueous phase with capsule suspension injection. Experiment performed using device "d". 


\section{List of Abreviations and Symbols}

IOR - Improved oil recovery

EOR - Enhanced oil recovery

CLSM - Confocal laser scanning microscopy

PV - Porous volume

$\mathrm{O} / \mathrm{W} / \mathrm{O}$ - Oil in water in oil double emulsion

$\mathrm{W} / \mathrm{O} / \mathrm{W}$ - Water in oil in water double emulsion

PIV - Particle image velocimetry

PDF - Probability density function

PMT - Photomultiplier tubes detector

HyD - Hybrid detector

$\phi$ - Porosity, percent $V_{t}$

$\phi_{i}$ - Porosity calculated by image acquired, percent $V_{i}$

$\rho_{w}$ - Water density, $\frac{\mathrm{kg}}{\mathrm{m}}$

$\sigma$ - Interfacial or surface tension, $\frac{J}{m^{2}}$

$\theta$ - Contact angle, degrees

$\mu_{o}$ - Oil viscosity, mPa.s

$\mu_{w}$ - Water viscosity, mPa.s

$k_{w}$ - Effective permeability to water, $\mathrm{mD}$

$V_{t}$ - Total device volume, $\mu \mathrm{l}$

$V_{p}$ - Porous volume, $\mu \mathrm{l}$

$V_{i}$ - Acquired image volume, $\mu \mathrm{l}$

$P_{b}$ - Pressure on the beginning of experiment, atm

$V_{b}$ - Volume on the beginning of experiment, 1

$P_{e}$ - Pressure on the end of experiment, atm

$V_{e}$ - Volume on the end of experiment, 1

$S_{w i}$ - Initial water saturation, percent $V_{i}$

$S_{w}$ - Water saturation, percent $V_{i}$

$S_{o}$ - Oil saturation, percent $V_{i}$

$S_{\text {or }}$ - Residual oil saturation, percent $V_{i}$

$C a$ - Capillary number

$Q_{i}$ - Flow rate of the inner phase, $\mu \mathrm{l} / h$

$Q_{m}$ - Flow rate of the middle phase, $\mu \mathrm{l} / h$

$Q_{o}$ - Flow rate of the continuous phase, $\mu \mathrm{l} / h$

$d_{i}$ - Inner diameter of the injection capillary, $\mu \mathrm{m}$

$d_{c}$ - Inner diameter of the collection capillary, $\mu \mathrm{m}$

$d_{s}$ - Diameter of the sintered glass spheres, $\mu \mathrm{m}$ 
"It's not what you look at that matters, it's what you see."

Henry David Thoreau . 


\section{Introduction}

\section{1}

\section{Motivation}

In spite of the diversification of energy sources witnessed in the last decades (solar power, biofuels, wave energy, geothermal power, wind energy, and others), fossil fuels probably will stand as a major energy source for a long while [24]. Thus, advances in making oil and gas production more efficient will continue to promote a major impact on the global economy for many years.

As these natural resources are finite, to keep their production, it is important to focus the technological advances on new fields discoveries and on improvements in their production efficiency.

In the early life of an oil field, its production may occur using the natural energy of the reservoir; this is called primary recovery. However, the reservoir energy can rapidly decrease, reducing the recovery efficiency. Thus, primary recovery is mostly used in gas fields or in field evaluation campaigns. So, to maintain the oil reservoirs in good production conditions, the most common method is the injection of water into the reservoir, called secondary recovery. Water injection keeps the reservoir pressurized, pushing the oil toward production wells. However, even increasing oil production, water injection can create paths in the porous reservoir through which the water will move preferentially. Thus, a large amount of oil can remain trapped in the reservoir that could still be produced using enhanced oil recovery (EOR), or tertiary, methods.

Aiming the recovery of immobile oil after water injection, a promising method is the emulsion injection. The emulsion penetrates the porous media and eventually blocks the water preferential paths, forcing the water to displace the oil that was trapped in different regions. Figure 1.1 (adapted from [1]) presents a diagram of two pores being blocked by a group of emulsion droplets, forcing the water to move through the path represented by red arrows. 


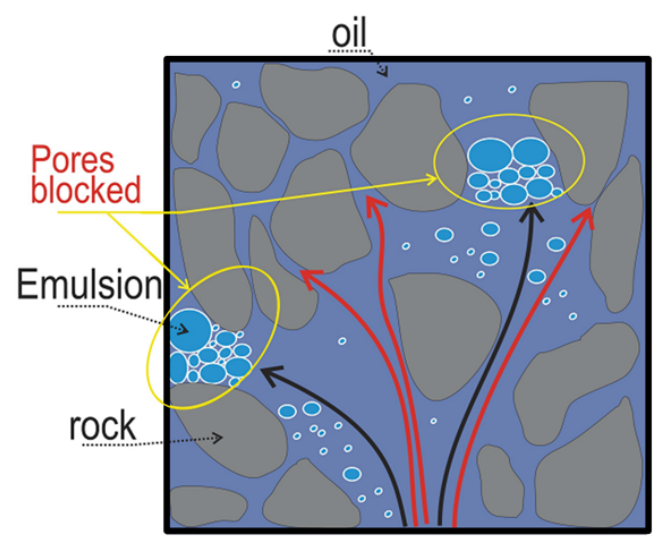

Figure 1.1: Emusions blocking the preferential path. Adapted from Engelke [1].

The injection of microcapsules also works by blocking pores of the water preferential path, increasing the pressure inside the porous media and diverting water, thus displacing the trapped oil. The main difference between emulsion and suspension of microcapsules for EOR is that when working with microcapsules, it is possible to control the mechanical properties of the blocking object. Basically, one can vary the mechanical properties on combining a fixed diameter with a specified shell thickness and shell material properties.

Emulsion injection has already been tested in a field [9], but so far there seems to be no application of microcapsules suspension technique for oil recovery. So, before performing a field test of microcapsules injection, it is important to comprehend how, on a micro scale, the capsules behave on a porous media. A common method to study interaction on micro scale is using two dimension (2D) microfluidic porous devices, similar to the one used by Gabriel Cruz [25] to analyze emulsion efficiency on oil recovery.

However, for a better understanding of how the microcapsules work it would be interesting to verify how they behave in a three dimensional (3D) porous device since $2 \mathrm{D}$ porous devices do not show the complex pore connectivity of a porous rock.

So far, the major part of the works published on 3D experiments on oil recovery uses tomography techniques to visualize phase arrangements inside a porous media [26] [27] [28]. However, using these techniques it is not possible to obtain a clear phase distinction and to perform a dynamical analysis of the fluids' motion. Thus, in this work, confocal laser scanning microscopy (CLSM) was used to analyze the effects and the behavior of the microcapsules inside the porous media.

The first work reporting the application of CLSM for multiphase flow through a porous media was from Datta [20], which performed different experiments aiming to observe and quantify two phase displacement phenomena. 
The author managed to measure fluid velocities and volumes occupied by each phase. The work from Vimieiro Júnior [13] followed Datta's procedures, introducing a third phase, that consisted of microcapsules with a polymeric (PDMS) shell composition, hoping to investigate a possible oil recovery enhancement. A small recovery increase was obtained, but the results seem to be far from expected, probably because the PDMS capsules had a stiffness too high to penetrate the porous media.

In this work, following Vimieiro's experiments, an improved CLSM methodology was used to investigate the injection of softer microcapsules in a $3 \mathrm{D}$ porous device as an EOR method.

\section{2}

\section{Dissertation goals}

The main objective of this work was, using visualization by CLSM, to perform experiments with the injection of a new softer microcapsules (compared to Vimieiro's) to promote enhanced oil recovery.

Additionally, a series of new features was implemented in order to achieve a step forward in the use of CLSM to visualize and quantify three phase flows on $3 \mathrm{D}$ porous devices:

- Using more fluorescent pigments (allowing the visualization of two separated phases and the capsules simultaneously);

- implementing new software tools to extend the depth of visualization into the device;

- building new porous devices (square tubes filled with an artificial porous media) with thinner walls for better visualization and more precise experiments;

- building devices with different porous properties (homogeneous porous media and two-layered porous media);

- building a new porous device holder, that made possible to rotate the porous media device and visualize it from different sides. 


\section{3 \\ Scope of work}

This work is structured in four chapters, besides this introduction. The theoretical frameworks are presented in Chapter 2, where some aspects of enhanced oil recovery, microcapsules production, confocal microscopy and image processing are reviewed. Chapter 3 describes how the experiment was carried on. This chapter was divided into three parts: experiment design; experimental procedure; and data processing routines. The results and their analysis are presented in Chapter 4. Finally, Chapter 5 is reserved for the conclusions and suggestions for future works. 


\section{2}

\section{Theoretical Framework and Literature Review}

This chapter presents the theoretical principles and a review of previous work done in the main topics that serve as a base to this thesis. It is divided into five parts: Flow in porous media; Enhanced oil recovery; Capsules as a recovery method; Confocal microscopy and Image processing.

\section{1}

\section{Flow in porous media}

A porous medium is a solid structure with some small empty spaces. These empty spaces are called pores, which can be connected among themselves or isolated. When one or more fluids are moving through a porous media, the description of the flow phenomena needs a special treatment.

The physics of flow happens in the microscale channels formed by the pores. However, it is almost impossible to use the microphysics flow equations to describe the macroscopic behavior of fluid motion through the whole porous medium. Then, what is normally done, is to change the scale of analysis, averaging the microscopic flow behavior to a larger scale. To deal with this problem, the Darcy's Law, Eq. 2-1, is the most common scaling method. It relates the fluid flow rate in a porous medium as shown in 2.1 to a parameter called permeability, $K$.

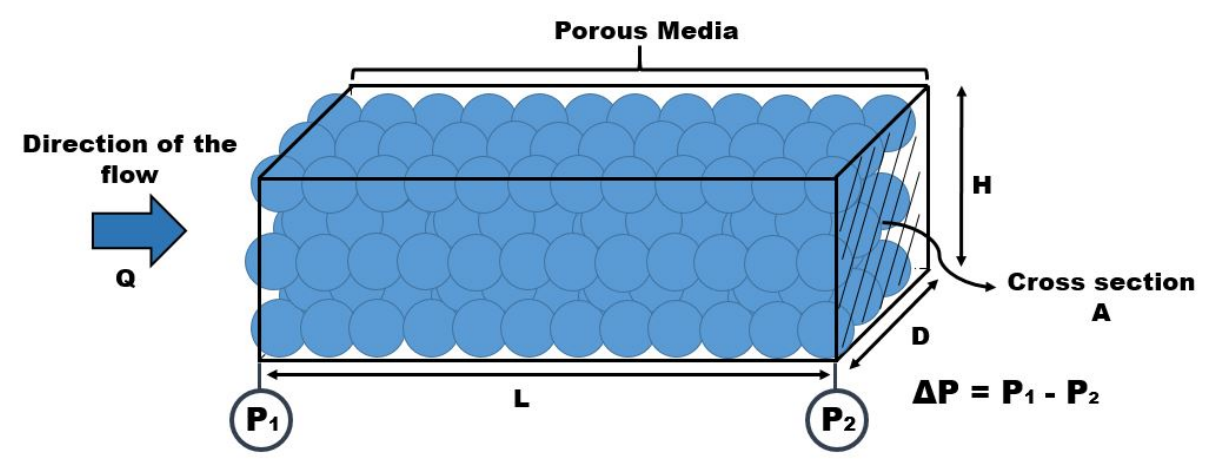

Figure 2.1: Diagram of a flow through a porous media.

$$
\frac{Q}{A}=\frac{K}{\mu} \frac{\Delta P}{L}
$$


where $A$ is the cross section of the porous medium and $\frac{Q}{A}$ is the Darcy's velocity. Important to notice that the Darcy's velocity is not the velocity of the fluid through the porous channel. Despite its dimension, it is not a true velocity, but rather a volume averaged velocity, computed as the ratio of the fluid volume flowing per unit of transversal area, including the solid and void spaces. In Eq. 2-1, $\frac{\Delta P}{L}$ is the pressure gradient through the porous medium and $\mu$ is the fluid's viscosity.

The permeability measures the conductivity of a fluid through a porous medium. It depends on properties of the porous medium such as porosity, specific surface area and pores structure, that will be defined next.

The most intuitive parameter to define a porous medium is the porosity. Porosity, $\phi$, is the ratio of the volume occupied by the empty pores, $V_{p}$, and total volume of the sample, $V_{t}$, as shown in Eq. 2-2.

$\phi=\frac{V_{p}}{V_{t}}$

Notice that the solid volume, $V_{s}$, and the porous volume compose the total volume.

$V_{t}=V_{s}+V_{p}$

There are different techniques to measure the porosity of a porous medium, as can be seen in [29]. The one used in this work was the imbibition method, where the weight of the porous medium is measured when it was dry and compared with the weight after the medium is filled with a liquid, in this case, water. Then, using the difference of weight $(\Delta m)$ of water, the porous volume can be calculated according to Eq. 2-4.

$V_{p}=\frac{\Delta m}{\rho_{w}}$

where $\rho_{w}$ is the water density.

Another porosity measurement method that was also used in this thesis is by image processing. After a 3D microscopic image of the porous medium was obtained and processed, it was possible to distinguish the solid structure from the pores and pore throats, by the use of proper fluorescent pigments. Then, by counting voxels, it is possible to calculate the volumes and then calculate porosity by eq. 2-2. A similar method is commonly used to determine the porosity from core samples using a computed tomography scan [30] [31] [32] 
[33] [34].

Table 2.1, obtained by Sahimi [22], present the ranges of porosity normally found on different types of porous media.

Table 2.1: Table obtained by Sahimi [22]. Porosity range of different types of porous media.

\begin{tabular}{cc}
\hline Porous medium & $\phi(\%)$ \\
\hline Black slate powder & $57-66$ \\
Silica powder & $37-49$ \\
Random packing of spheres & $\mathbf{3 6}-\mathbf{4 3}$ \\
Sand & $37-50$ \\
Sangstone & $8-38$ \\
Limestone (dolomite) & $4-10$ \\
Coal & $2-12$ \\
Concrete & $2-7$ \\
\hline
\end{tabular}

The specific surface area, $S a_{s}$, is also an important parameter that has a strong effect on the permeability analysis. It is possible to conclude, by comparing two porous media with same porosity but different superficial area, $S a$, that the one with higher surface area will have a lower permeability. The specific surface area is described by Eq. 2-5.

$S a_{s}=\frac{S a}{V_{t}}$

where $S A$ is the total surface area of the porous medium.

Other important parameters that affect flow through a porous medium are the interfacial tension, wettability and capillary pressure. These concepts will be specially relevant when more than one fluid are flowing through the porous medium (multiphase flow).

At the interface between two materials, molecules are exposed to a different environment than those inside the materials. In the bulk of a material, the cohesive forces between its molecules balance themselves. However, near the interface, there is a resultant force due to the magnitude of the forces between the molecules of the two materials, different than those found between the molecules of each material. This phenomenon, illustrated in Fig. 2.2, gives rise to the interface configuration supported by a binding energy. It is intuitive to realize that the shape of this equilibrium interface will be spherical, so to minimize the surface energy. The interfacial tension, $\sigma$, is a property that 
quantifies this binding energy that forms a surface between two immiscible fluids.

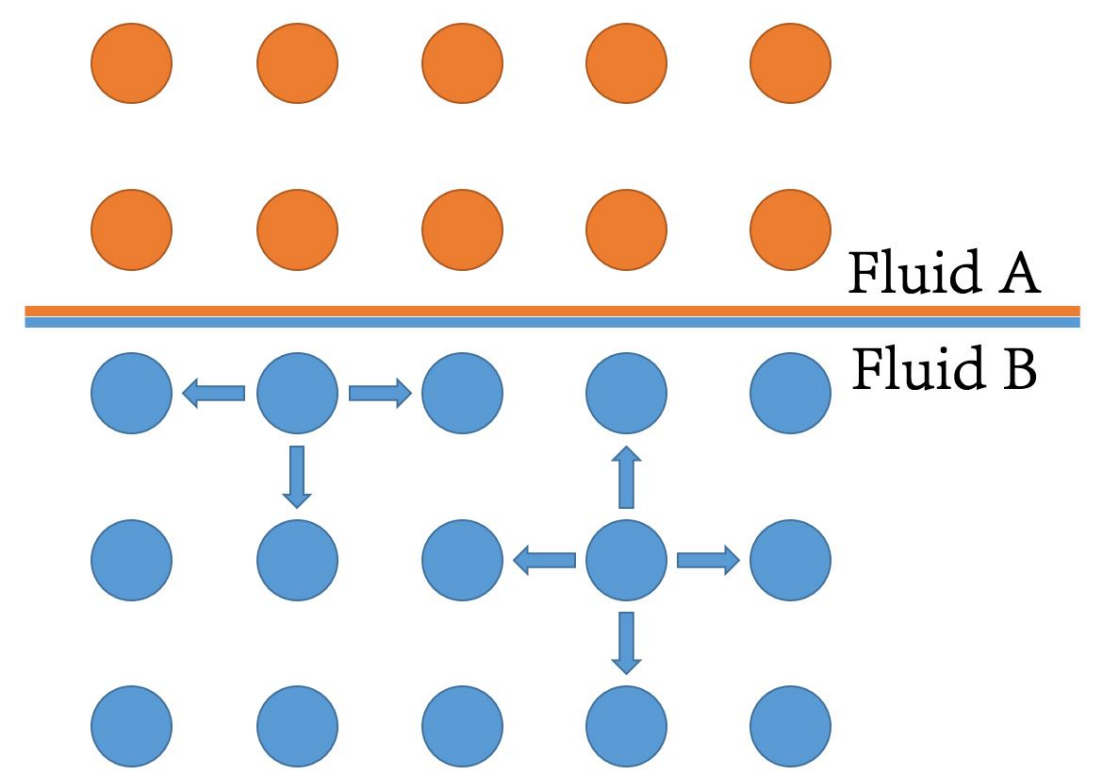

Figure 2.2: Illustration of the forces between molecules in the interface of two different materials.

In a formal way, the interfacial tension measures the surface energy density. So, if an amount of work, $\delta W$, is needed to increase the area of an interface by an amount, $\delta A$, it can be calculated as:

$\delta W=\sigma \delta A$

In general, the surface energy density associated with a solid or liquid interface against vacuum or gas is positive, since it is needed an amount of extra energy to increase its surface. Thus, for a given energy, the interface will look for the minimal area. This explains the fact that small bubbles and drops are spherical, as long as surface forces are much larger than gravity.

An interesting behavior occurs when two fluids and a solid are present together. According to the nature of the cohesive forces between them, a given solid will be wetted by one of the fluids. This is illustrated in Fig. 2.3, where two liquids, A and B, in contact with air (or another given fluid), touch a solid surface. Fluid A wets the solid surface, since it has more affinity with the solid than air; and this can be expressed by the angle formed by the three fluid contact. On contrary, Fluid B does not wet the solid surface, which rather has air as a preferred contact fluid. The angle formed between these fluids is named contact angle, $\theta$, and indicates the behavior of a two fluid-solid interface. 


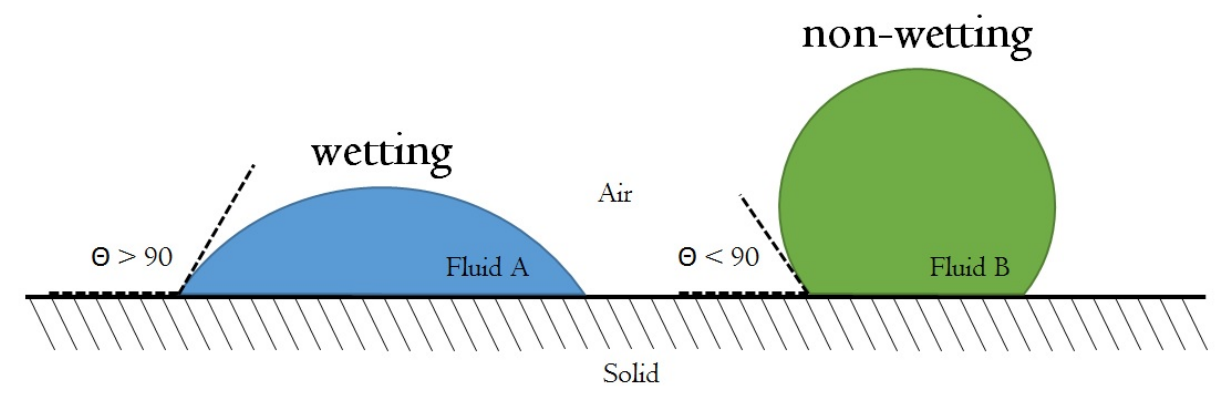

Figure 2.3: Illustration of wetting and non-wetting fluid configuration.

In fact, by solving a force balance at the interface between the two liquids and the solid, it is possible to calculate the contact angle as a function of the interfacial tensions between the solid and each fluid and the interfacial tension between the fluids. This relation is known as the Young Equation:

$$
\cos \theta=\frac{\sigma_{s-A}-\sigma_{s-a i r}}{\sigma_{A-a i r}}
$$

It is even possible that this force balance can not be obeyed, as in the case when $\sigma_{s-A}>\sigma_{A-a i r}+\sigma_{s-a i r}$, in such situation the value for $\cos \theta$ would be larger than 1, which is nonsense. In this case, physically, Fluid A completely wets the solid surface and simply spreads as a thin film of molecular thickness across the solid surface.

As a consequence of the wettability, a given fluid that wets a solid surface has the ability to penetrate into narrow channels of this solid, like those present in porous media. This phenomenon is clearly observed in an air-water interface in the presence of a small cylindrical glass tube, and it is known as the capillary effect. It takes place because the interfacial tension of glass in contact with air is larger than the interfacial tension of glass in contact with water. Thus, as shown in Fig. 2.4, inside the tube the water reaches height, $h$, pushed by this difference of interfacial tensions. 


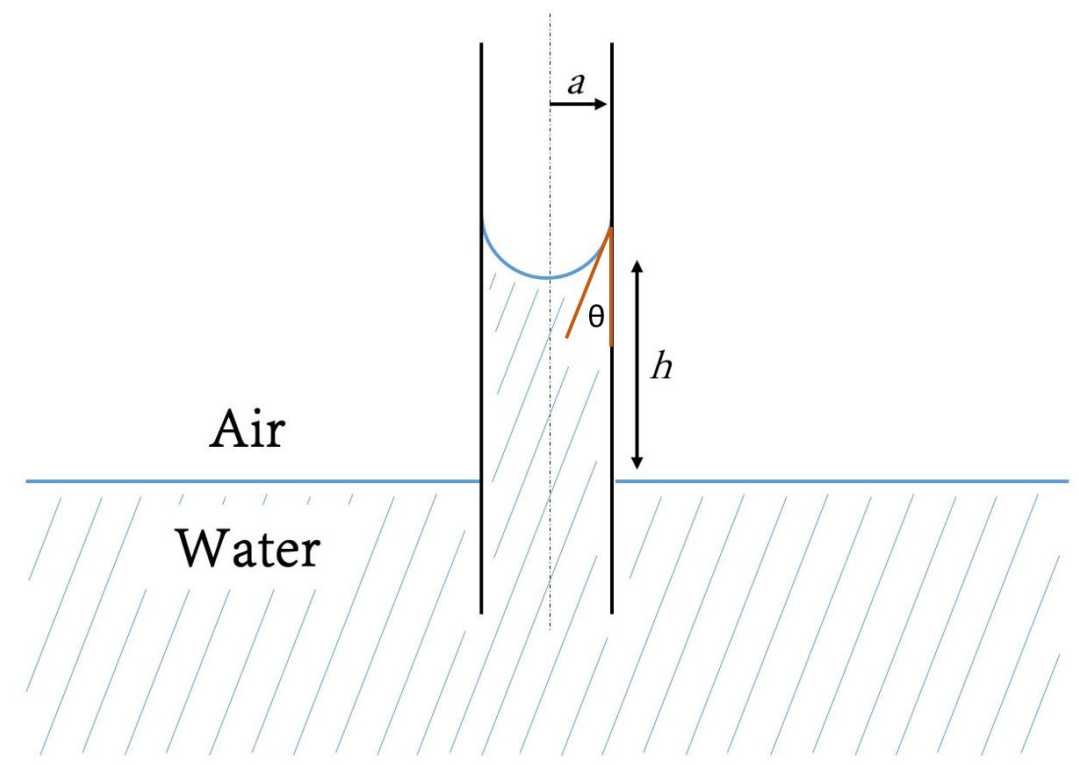

Figure 2.4: Illustration of the capillary effect on water wetting the cylindrical glass tube.

By a force balance, if $a$ is the radius of the tube, it is possible to calculate the capillary rise, $h$ :

$\left(\sigma_{s-a i r}-\sigma_{s-w a t e r}\right) 2 \pi a \approx \rho_{w} g \pi a^{2} h$

Or, by applying the Eq. 2-7:

$h=\frac{2 \sigma_{\text {air-water }}}{\rho_{w} g a} \cos \theta$

When a fluid displaces another inside a porous medium (as in oil recovery by water injection), the wettability of the solid surface is a very important parameter to forecast the amount of fluid that can be displaced. Obviously, a displacing fluid that wets the surface favors the invasion of small pores. Thus, whenever the flow happens in small microchannels, like those found in oil reservoirs, wettability plays a key role in the recovery, as illustrated by Fig. 2.5 from Lenormand [2]. The distribution of the fluids in the pore space is governed by the contact angle of the system, given the role of capillary effects. 


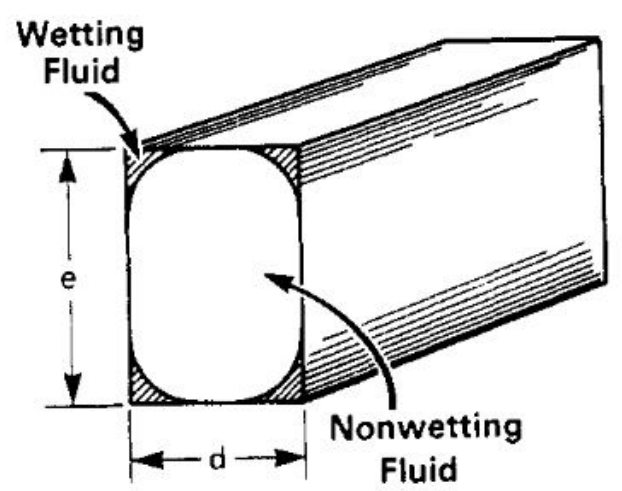

Figure 2.5: Non wetting fluid flow through a porous channel. Image by Lenormand [2].

Of special interest in this work is the multiphase oil-water flow through porous channels. These porous channels are small enough so that capillary forces may be compared to viscous forces of the flow, when one of the phases displaces the other with a velocity $v$. If $\sigma_{\text {oil-water }}$ is the surface tension between oil and water and $\mu$ is the viscosity of the displacing phase, the Capillary Number, $C a$, can be defined as:

$$
C a=\frac{v \mu}{\sigma_{\text {oil-water }}} .
$$

Using Darcy's velocity, it is possible to rearrange the Eq. 2-10:

$$
C a=\frac{K}{\sigma_{\text {oil-water }}} \frac{\Delta P}{L} .
$$

However, in this work the first formulation of $\mathrm{Ca}$ was used as it was not possible to measure the pressure drop in the experiments.

Flow in porous media happens in many natural and industrial processes and studying it can help to solve problems in different fields, such as underground water and oil recovery, drug delivery in tissues, food industry, textiles, foams and many others.

This work will investigate a technique applied to improve oil recovery.

\section{2}

\section{Oil recovery}

Improvements on oil recovery efficiency is a major area of study in Petroleum Engineering, aiming to maximize the exploitation of this natural resource. It is common to classify oil recovery processes in three phases: 
primary, secondary and tertiary; sometimes one of these phases can be skipped in industrial applications.

An important parameter to discuss the efficiency of oil recovery is the Recovery Factor, $R F$, defined as the ratio of the volume of oil recovered from a porous medium by the total volume of oil present in this porous medium:

$R F=\frac{\text { Volume of oil recovered }}{\text { Volume of oil in the porous medium }}$

\subsection{1}

\section{Primary and secondary oil recovery methods}

The primary recovery process makes use of the reservoir's own energy. It is usually applied to fields that have a higher concentrations of natural gas, or in brief campaigns for field evaluation. Alargoni [35] describes five drive mechanisms for the primary recovery: solution gas drive; gas cap drive; water drive; gravity drainage; mixed drive. However, oil reservoirs can rapidly loose their energy when kept only in primary recovery, resulting in fast production decline and low $R F$. Usually, since it contributes to a rapid depletion of oil reservoirs, primary recovery is skipped and the exploitation starts by secondary recovery.

Secondary oil recovery is based on the idea of maintaining, or even increasing, the reservoir pressure by injecting water or gas into the reservoir through wells. This is done by keeping the total fluid volume inside the porous media: as oil is removed, water or gas is injected. At the same time, the water or gas injected at the proper reservoir point help to displace the oil toward the production well, sweeping the reservoir.

Secondary oil recovery needs a good knowledge of the reservoir properties and their spatial distribution, as well as of the configuration of the fluids inside it, in order to position the optimal locations for production and injection wells.

In any case, secondary recovery cannot completely sweep all the oil in the reservoir. Phenomena previously described, such as wettability and capillarity, will play an important role on the phases configurations after the sweep. Also, hydrodynamic instabilities, related to the viscosity differences and displacement speed will effect the amount of oil that can be recovered. So, unfortunately, as described by Sahimi [22] "fifty percent or more of the original oil-in-place is left behind in a typical oil reservoir after the primary and secondary recovery processes end." To further discuss these aspects, is convenient to define the saturation of a fluid $i$ in a porous medium, $S_{i}$, as the volumetric fraction occupied by this fluid in the porous medium: 
$S_{i}=\frac{V_{i}}{V_{p}}$

Notice that if only two fluids, $i$ and $j$, occupy a porous medium:

$S_{i}+S_{j}=1$

When two-phases flows in a porous medium, they compete between themselves for preferences in flowing channels. Thus, the single phase based concept of permeability, $K$, has to be corrected so to reflect this competition. Then comes the concept of relative permeability: according to the saturation of a phase, its relative permeability will multiply the right hand side of Eq. 2-1, that will become:

$\frac{Q_{i}}{A}=k_{r i} \frac{K}{\mu_{i}} \frac{\Delta P}{L}$

Equation 2-15 is the expression for the Darcy's Law when two fluids flow in a porous medium. It allows to calculate the flow rate of fluid $i, Q_{i}$, as a function of its relative permeability, $k_{r i}$, and its viscosity, $\mu_{i}$.

The relative permeability is an experimentally determined function for two fluids in porous medium, and it is a function of the saturation of the fluids. Figure 2.6 shows a typical behavior of the relative permeabilities of a oil-water system in a porous medium.

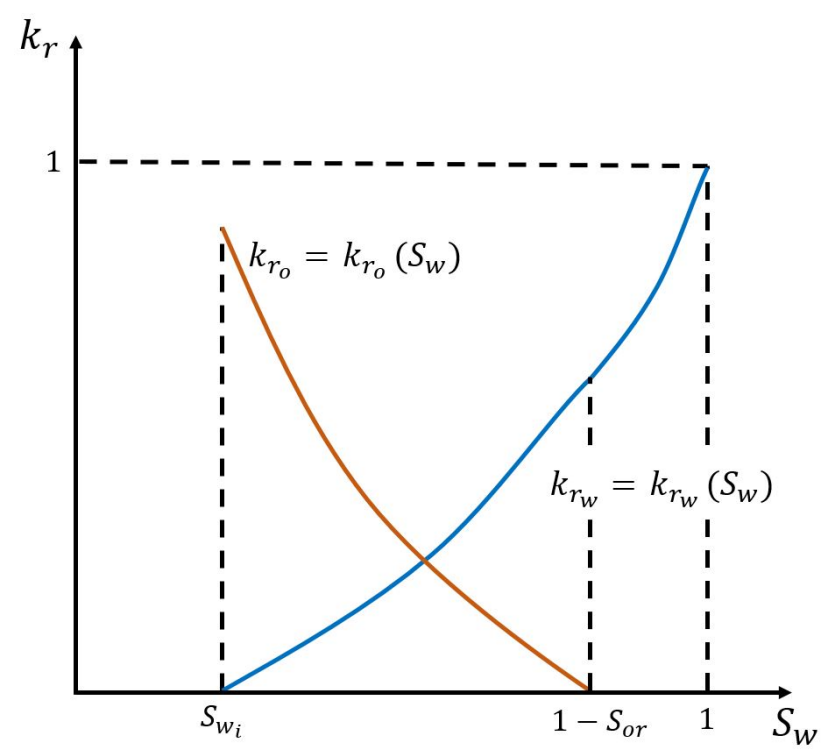

Figure 2.6: Typical relative permeability curve. 
For a given saturation, one can determine $k_{r o}$ and $k_{r w}$. However, capillary effects impose limits to relative permeabilities. The curves show that its not possible to have water flow through the porous media when $S_{w}$ is below the irreducible water saturation, $S_{w i}$. On the other side, defining $S_{o r}$ as the residual oil saturation, no oil flow will be observed if the water saturation is above $\left(1-S_{\text {or }}\right)$.

Another reason of the relatively low efficiency of the secondary recovery is a flow instability that occurs when two fluids of different viscosities move through a porous media, the viscous fingering instability. It is associated to the displacement of a fluid by another with smaller viscosity and may happen in other geometries than porous media, such as Hele-Shaw cell. It is possible to observe viscous fingering both with miscible or immiscible fluids. An extensive review of the fingering phenomenon was presented by Homsy [36].

Homsy's review work describes the physics of the flow instability, that is observed on secondary and tertiary oil recovery. The viscous fingering effect is due to the displacement of a higher viscosity fluid, $\mu_{2}$, by a lower viscosity fluid, $\mu_{1}$. The viscosity ratio, $M$, is defined by Eq. 2-16. The larger viscosity ratio, more intense fingering will be. Also, there is a critical velocity above which fingering will start, so fingering may not happen at very slow injection rates.

$M=\frac{\mu_{2}}{\mu_{1}}$

The work done by Chuoke et al. [3] shows an experimental result of the oil displacement by water injection in transparent powder pack models. Two models were used, with dimensions of $5 \times 30 \times 60 \mathrm{~cm}$ and $2 \times 9 \times 18 \mathrm{~cm}$. The pack was made of Pyrex glass powder and initially saturated completely with oil. Two of his experimental results are presented on Fig. 2.7, and illustrate the fingering phenomenon. As water injection starts in the porous media, a planar interface starts the displacement. However, this planar front is unstable when the less viscous fluid pushes the other and eventually a small finger breaks the planar front. Since the viscosity in the area around the small finger will be smaller than in the rest of the front, a preferential path is established, increasing continuously the length of the finger.

An interesting aspect is that a new planar front is established in the lateral side of a finger. Eventually, in a smaller scale, a new finger may break through this lateral side, running sort of parallel to the first front. Thus, fingering may be viewed as a fractal phenomenon that creates a network of preferential paths of the less viscous fluid, leaving behind a large amount of 
the more viscous fluid.

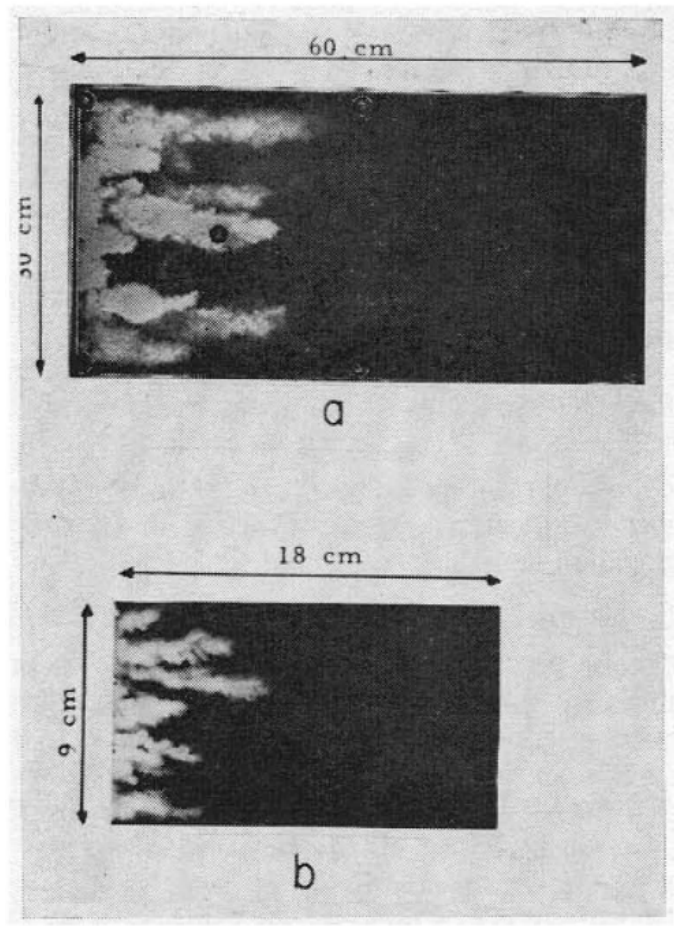

Figure 2.7: Image adapted from Chuoke et al. [3]. Two examples of water displacing oil and forming the viscous fingering pattern. (a) $\mu_{o}=9.45 \mathrm{cP}$, $K=96 D, \mu_{w}=1 \mathrm{cP}, U=0.00932 \mathrm{~cm} / \mathrm{s}$ and $\sigma=42$ dyne $/ \mathrm{cm} ;$ (b) $\mu_{o}=66$ $c P, k=65 D, \mu_{w}=0.936 c P, U=0.0106 \mathrm{~cm} / \mathrm{s}$ and $\sigma=48$ dyne $/ \mathrm{cm}$.

A study by Lenormand's [2] focus on the different configurations observed when injecting a fluid into a porous medium saturated with another, immiscible with the one injected. It focus both on drainage (when the fluid injected does not wet the porous medium) and on imbibition (when the fluid injected wets the porous medium). The results show experiments with fluids with different viscosities and at different flow rates. So, its results considers both capillary and viscous effects.

Starting by describing the physical mechanisms that happen at the pore level, Lenormand designed experiments to identify patterns where stable and unstable displacement happens, both in imbibition as in drainage, and correlated this patterns with $C a$ and $M$.

Lenormand showed that fingering may happen caused also only by capillary effects, in cases where viscous forces are negligible. This occurs due to the percolation effects through meniscus, typically at small values of $C a$. For situations where viscous forces are relevant, the flow may assume a stable configuration when the viscosity of the injected fluid is higher than the viscosity of the displaced fluid. 
So, considering the capillary and viscous forces that are present in two phase flow in a porous medium, one can understand that secondary oil recovery will not be able to fully displace the oil from the rock. This confirms the words of Sahimi [22], mentioned earlier in this section, saying that more than half of the total amount of the oil will remain in the reservoir after secondary recovery.

In practice, these effects will lead to the creation of preferential paths for water inside the porous rock, leaving behind a large amount of oil as sketched in Fig. 2.8, adapted from Kantzas et al. [4].

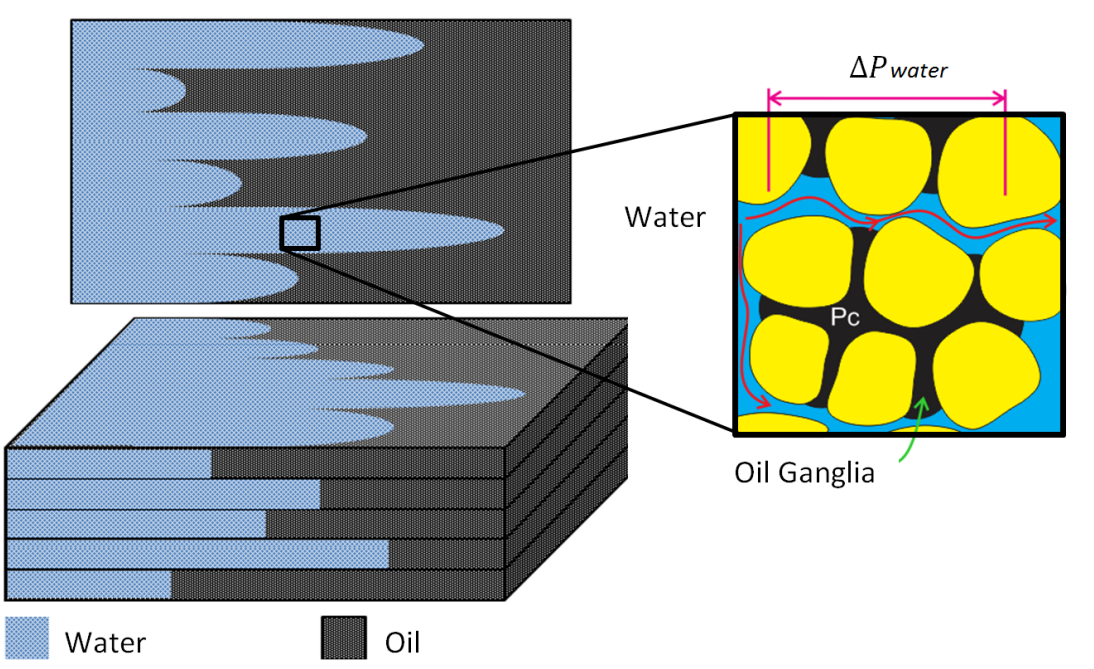

Figure 2.8: Adapted from Kantzas et al. [4]. Illustration of viscous fingering effect on the injection of water in a porous medium. The preferential path is formed and, even in the area that the water sweep the oil, some ganglia is left by the capillary pressure.

Even in the regions swept by the water phase, a certain volume of oil remains trapped by capillary forces. In a 1982 review, Payatakes [6] discusses the dynamics of oil ganglia during immiscible displacement in water-wet porous media. The work describes how a ganglion, initially at rest, can be deformed and eventually mobilized by the application of a pressure due to the flow of a displacing fluid. It also correlates the startup of the ganglion motion (mobilization) and ganglion break up with values of the Capillary Number.

The relation of oil mobilization and, consequently, the decrease on residual oil saturation by the increase of the Capillary Number were studied by Datta et al. [5] using a visualization technique similar to the one used in this work. Figure 2.9, obtained by Datta et al. [5], shows that decrease of the normalized $S_{\text {or }}$ curve. Datta et al.'s work will be further discussed in this chapter. 


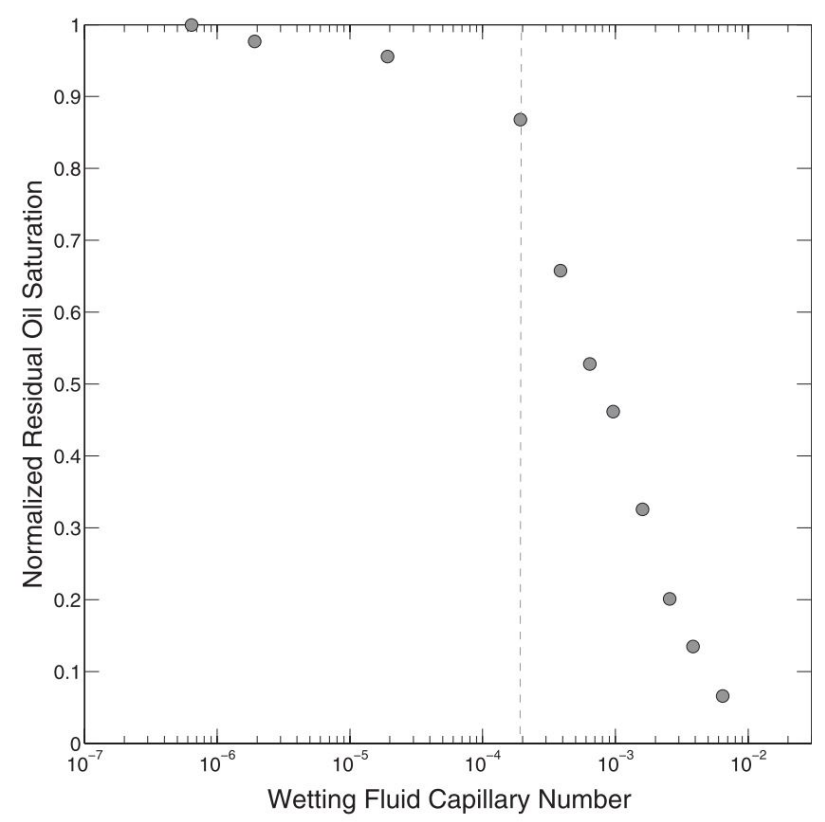

Figure 2.9: Normalized residual oil saturation measured using 3D confocal micrographs. Image from Datta et al. [5].

Some qualitative patterns of ganglion mobilization are presented by Payatakes. Figure 2.10, from Payatakes [6], shows the process of the mobilization of a ganglion initially at rest. There is a critical value for the pressure gradient (that can be correlated with $\mathrm{Ca}$ ) below what the ganglion will only show small deformations on its boundaries Fig. 2.10b. After reaching the critical value, the ganglion starts moving further following the flow Fig. 2.10c. 

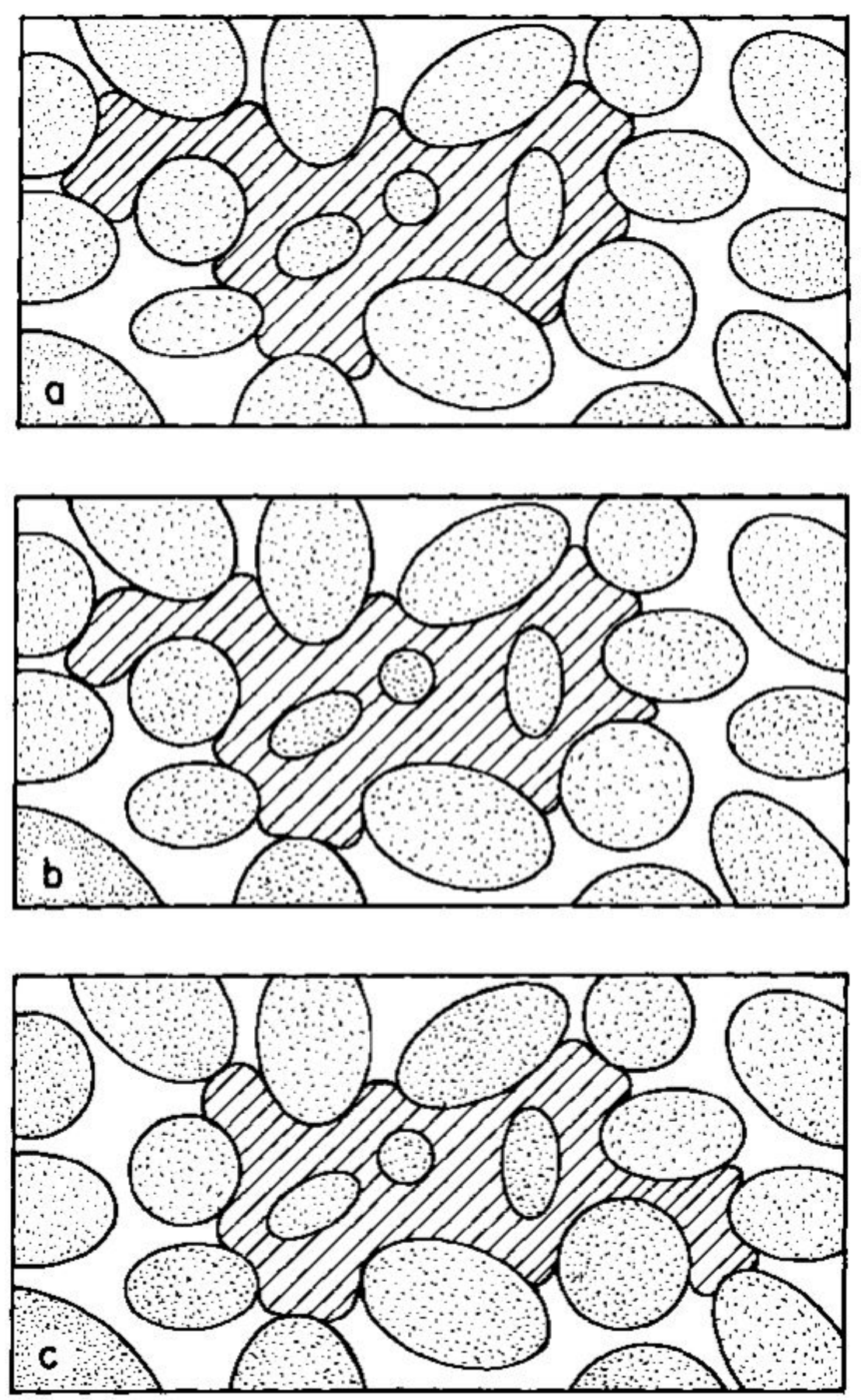

Figure 2.10: Illustration by Payatakes [6]. Oil ganglion behavior at (a) no flow; (b) aqueous phase flow from left to right, pressure gradient does not reach critical value; (c) the pressure gradient is higher than the critical value, ganglion was mobilized.

Payatakes also presents qualitative patterns of ganglion break up, or fission, usually into elongated ganglia, which were found by Hinkley [7] and showed on Fig. 2.11. 


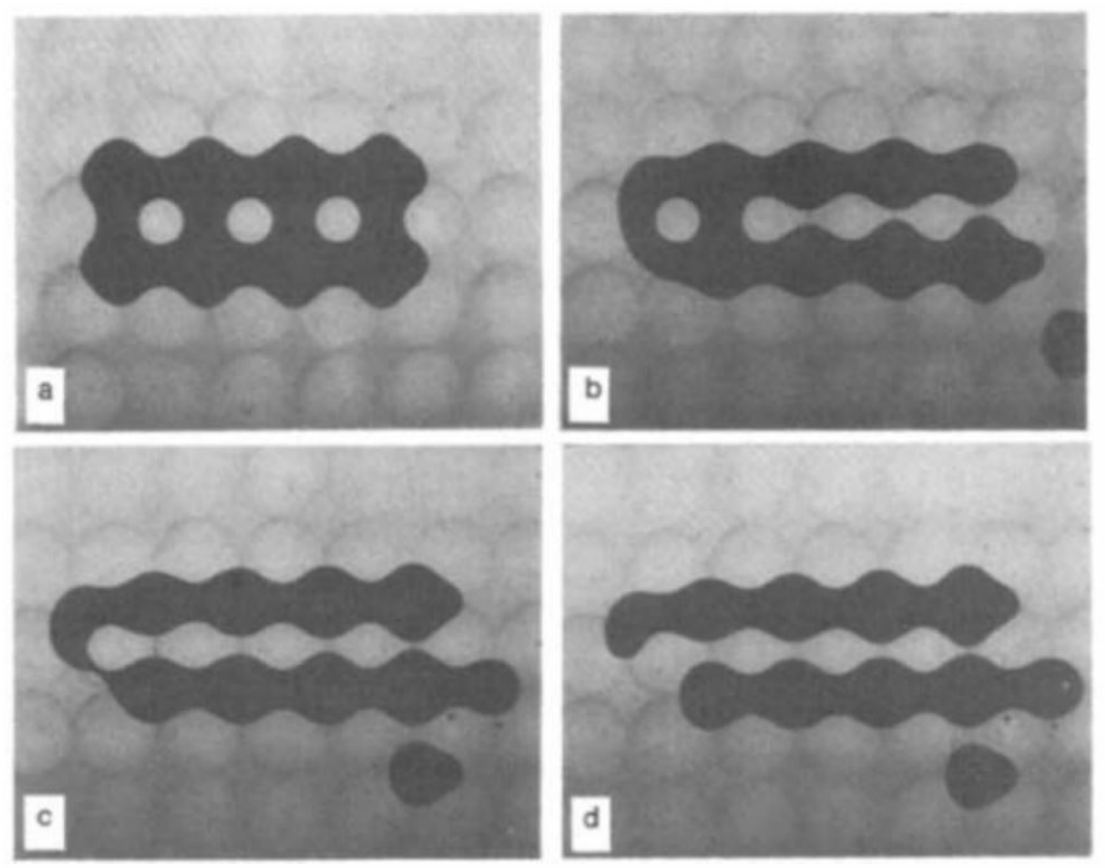

Figure 2.11: Images from Hinkley's [7] movie films. Movement of a ganglion at a porous medium, composed by glass beads, and different moments of the fission process.

\subsection{2}

\section{Enhanced oil recovery}

Thus, given the limitations of secondary recovery, to increase the efficiency of oil extraction, it is very important to study and develop tertiary recovery methods.

There are several types of enhanced oil recovery methods. An usual way to classify them is grouping according to the way they alter the flow condition. Olajire [8] suggest a classification in four types: thermal, gaseous, chemical or others, as show on the diagram Fig. 2.12.

Thermal methods work through increasing reservoir temperature and thus reducing oil viscosity or promoting steam distillation. Gaseous methods comprehends both miscible gas injection (and the gas acts as a solvent of the oil) and inmiscible gas injection (pretty much similar to water injection). Chemical methods aims to lower surface tension between oil and water. Other methods make use of mechanical and biological means to improve recovery. 


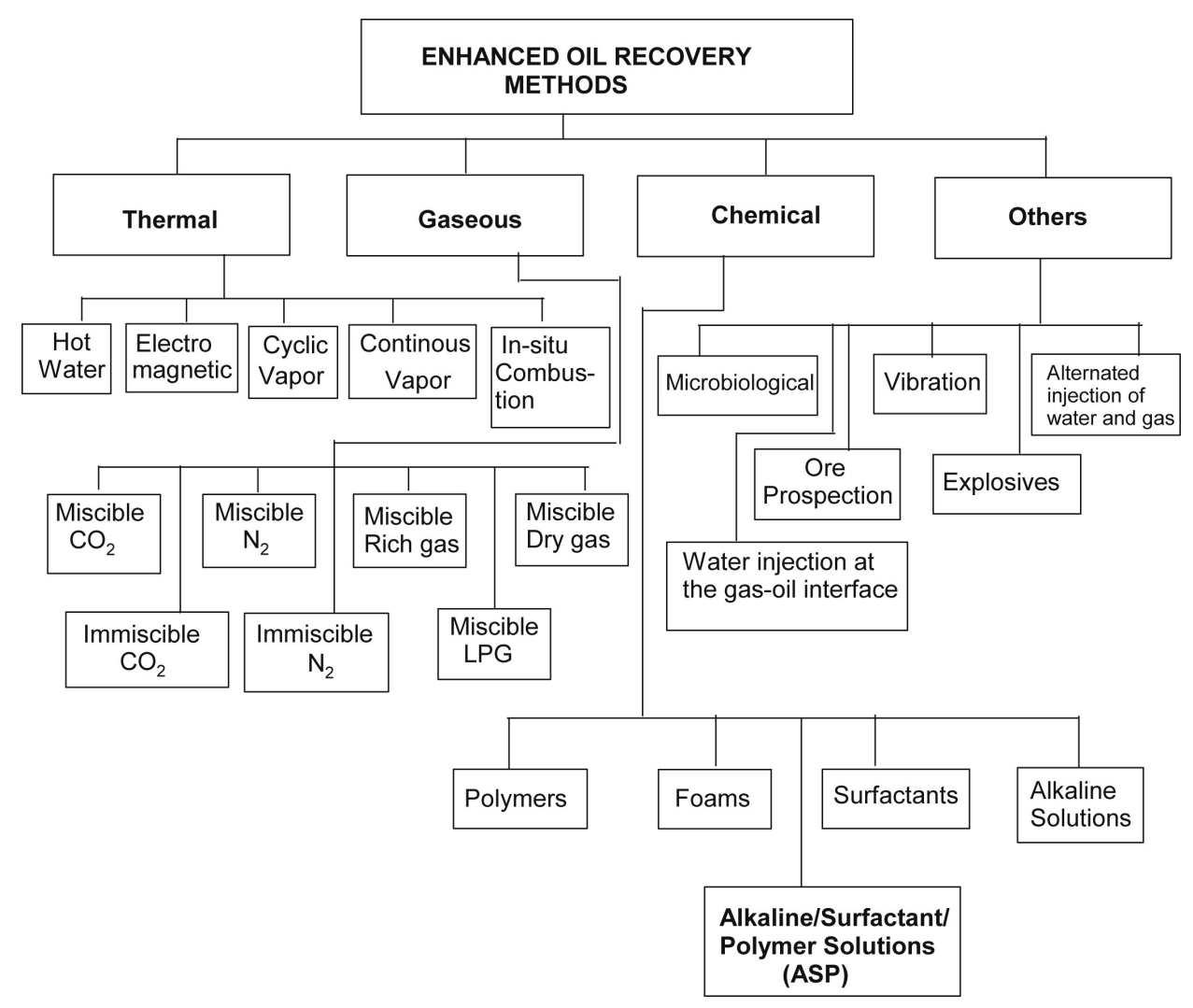

Figure 2.12: Diagram from Olajire [8] of some EOR processes.

Another way to group EOR is to divide on methods that act directly increasing the natural energy inside the reservoir and those that act by changing the interactions with the reservoir rock/oil system. The change of this second group could be a result of one or more of the following mechanisms: reduction of the interfacial tension between the displacing fluid and oil, increasing the capillary number, reducing capillary forces, increasing the drive water viscosity, providing mobility-control, creating oil swelling, reducing oil viscosity or changing the wettability of reservoir rock.

Given the scope of this study, a special focus will be placed on methods that work by interfering on the morphology of the oil-water flow in the porous medium, by injecting a large number of particles designed to block water preferential paths, increasing pressure gradient in front of oil ganglia mobilizing them. These methods, may be included in the others category in Fig. 2.12, and are strongly related to the role of emulsions injected into the reservoir.

As discussed by Thomas [37], it is argued that in all EOR methods emulsions are formed by the oil-water interactions. Sometimes emulsion formation could be assisted by thermal energy and rock materials or influenced by the chemicals present in the connate water and those in the injected fluid. It is also known that gas injection could provide the agitation needed to produce the emulsion drops. This emulsion production inside the porous media could 
have advantages or disadvantages on the oil recovery depending on the system behavior. Cases favorable to oil recovery occur when the emulsion droplets block high permeable paths, creating a pressure gradient that forces the fluids to follow different paths that could still contain some trapped oil, and by that increasing the oil recovery.

Considering this phenomenon, studies were performed to analyze the isolated effect of emulsion injection on oil recovery process. The work of McAuliffe [9] investigates the injection of emulsion in a core sample. After choosing two specific emulsion formulations based on their stability and drop size, he proceeded to inject these emulsions on a Boise core sample. First he injected an emulsion having medium droplet diameter of $1 \mu \mathrm{m}$. After injecting 10 porous volumes (PV) of emulsion into the core, the permeability was reduced from 1,600 to $900 \mathrm{mD}$. Then, the core was cleaned, saturated again with water and a larger drop, with average droplet diameter of $12 \mu \mathrm{m}$, emulsion was injected. After $9 \mathrm{PV}$ injected, the permeability was reduced to $35 \mathrm{mD}$. This permeability reduction can be seen as a good indicator that some pore channels were blocked by emulsions. Figure 2.13 shows the result of this experiment. The higher curve is the result from the injection of the first emulsion and the lower one is the permeability of the larger drop test.

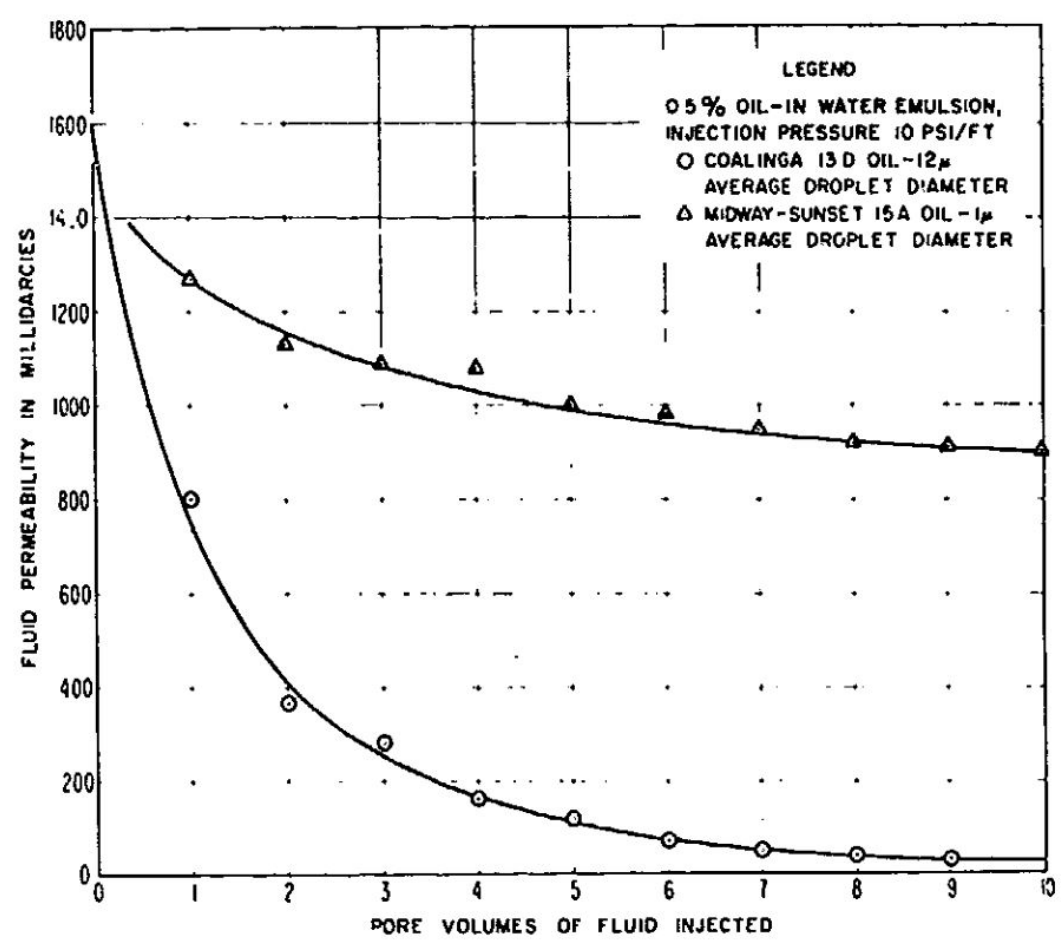

Figure 2.13: Result from McAuliffe [9], the difference on fluid permeability after the injection of emulsion with two different droplet sizes.

The work also tested a simultaneous emulsion injection through cores 
of different permeabilities mounted in a parallel set up. Figure 2.14 shows a diagram of the apparatus used on this part of the work.

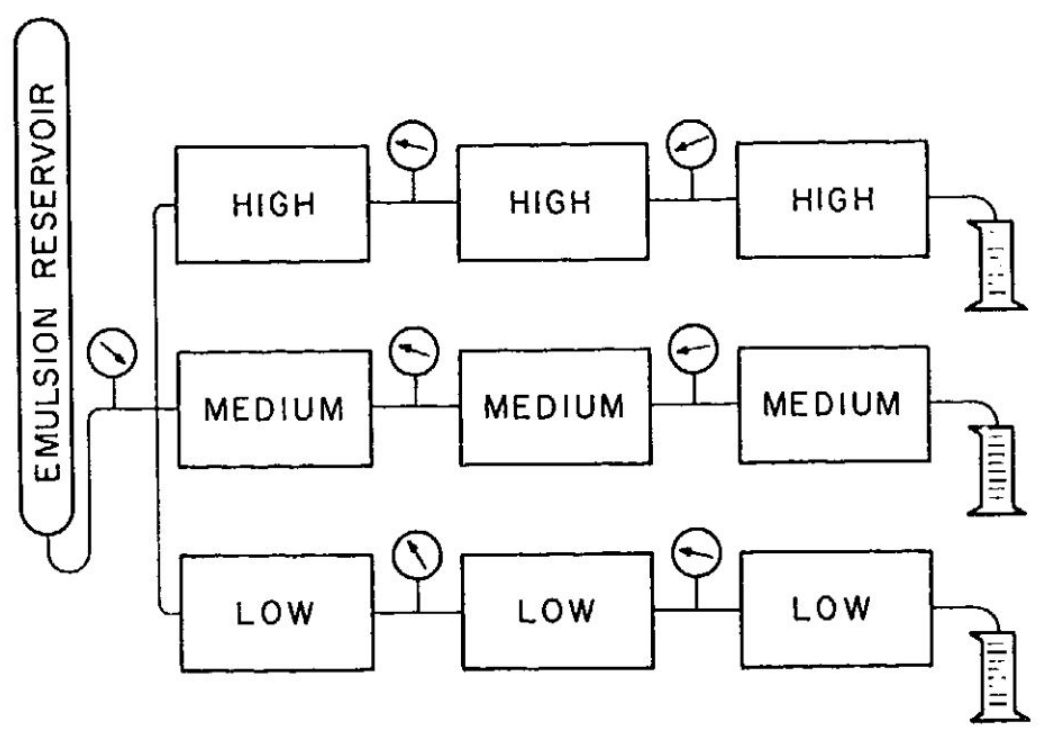

Figure 2.14: From McAuliffe [9], a schematic diagram of the experimental set up to test cores of different permeabilities mounted in parallel.

The result of this simultaneous emulsion injection was very interesting. The idea was that the oil-in-water emulsion would preferentially enter the more permeable cores, restrict flow in them, and thereby cause the fluids to flow into the others, less permeable, cores. By that, the sweep efficiency would be increased. In fact, the experiment showed results similar to the expectation.

Emulsion with small droplet sizes appeared in the outlet from all three sequence of cores. However, only the high permeability core outlet contained emulsion whose oil concentration was similar to the emulsion injected. Permeability of each core was measured, as presented on Table 2.2 , and it is possible to see that the high permeability core (Boise sandstone) presented the biggest reduction of permeability after the injection. By then, the three cores presented permeabilities very close to each other, as a result of the blockage by emulsion droplets. Thus, on the final part of the experiment, the flow was more evenly distributed between the cores. 
Table 2.2: Injection of emulsion through cores of different permeabilities mounted in parallel. By McAuliffe [9].

\begin{tabular}{|c|c|c|c|c|c|c|}
\hline \multirow{2}{*}{$\begin{array}{c}\text { Time } \\
\text { (minutes) }\end{array}$} & \multicolumn{3}{|c|}{$\begin{array}{l}\text { Core Fluid Permeability } \\
\text { (md) }\end{array}$} & \multicolumn{3}{|c|}{ Cumulative Pore Volumes } \\
\hline & High* & Medium** & Low** & High & Medium & Low \\
\hline 0 & 1,460 & 545 & 395 & 一 & - & - \\
\hline 1.02 & 670 & 240 & 245 & 0.26 & 0.15 & 0.13 \\
\hline 3.4 & 611 & 322 & 288 & 1.05 & 0.68 & 0.60 \\
\hline 8.6 & 312 & 211 & 212 & 2.10 & 1.63 & 1.45 \\
\hline 14.8 & 201 & 156 & 157 & 2.90 & 2.43 & 2.23 \\
\hline 27.4 & 136 & 61 & 64 & 5.50 & 3.87 & 3.72 \\
\hline 66 & 69 & 57 & 62 & 7.87 & 6.70 & 6.73 \\
\hline 101 & 57 & 42 & 51 & 9.84 & 8.75 & 9.29 \\
\hline 161 & 42 & 26 & 33 & 12.4 & 11.2 & 12.2 \\
\hline
\end{tabular}

Other experiments on flow of emulsion in porous media were developed by Thomas [37]. They focused on the characterization of in situ production of emulsion and on the analysis of oil recovery by the injection of emulsion. In one of the tests, crude oil was injected into a core initially saturated with water until establishing an initial oil saturation of $86.7 \%$. Then, $1.9 \mathrm{PV}$ of an emulsion was injected. Emulsion breakthrough happened at $0.88 \mathrm{PV}$ and at the end a recovery of $42 \%$ of the initial oil was obtained and the pressure drop increased from 55 to $280 \mathrm{kPa}$.

Cobos [10] proposed that to understand the physics of the phenomenon of enhanced oil recovery by blocking pores it was important to start from a simpler models before reproducing real field conditions. Thus, designed an experiment using a capillary tube with constriction and flowing through it different sizes of oil in water emulsion droplets (sizes comparable to constriction diameter). During the experiment, the pressured drop trough the capillary was measured. One of the results obtained by [10] is presented in 2.15 . 

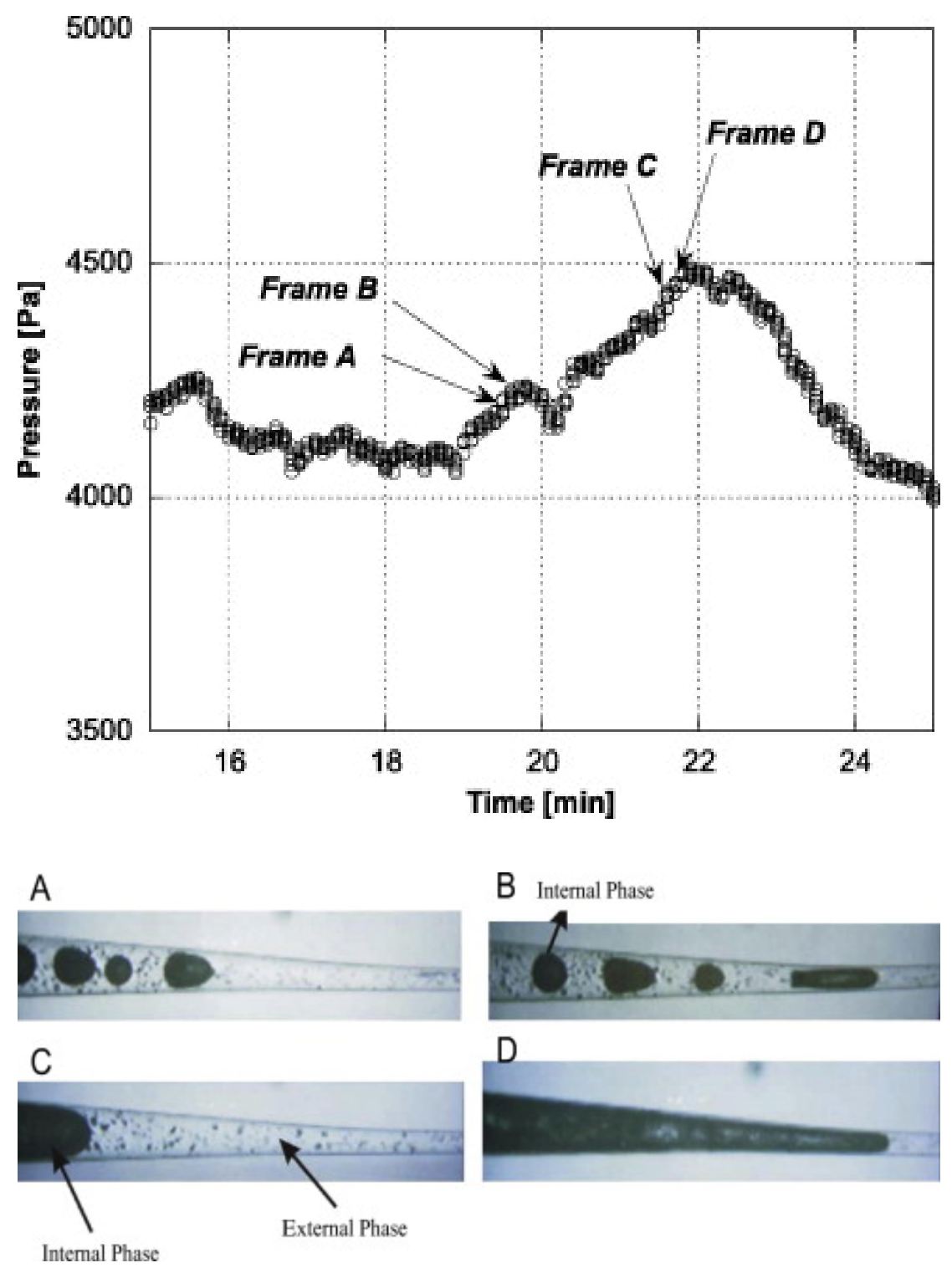

Figure 2.15: From Cobos [10]. Evolution of the inlet pressure at $\mathrm{Q}=0.04 \mathrm{ml} / \mathrm{h}$ of a larger emulsion and images of the flow near the capillary throat at the instants highlighted in the plot.

Figure 2.15 show that the smaller droplets had less effect on the increase of the pressure drop comparing to the passage of the larger emulsion droplets. The deformation of the small droplets due to the constriction, from Frame A to Frame B, increased the pressure drop. After that, the passage of a larger emulsion droplet, Frame $\mathrm{C}$ and D, is the reason for the larger increase of the pressure drop.

Another work done by McAuliffe [11] brings real field scale results for the injection of oil-in-water emulsion into the Midway-Sunset field, in California. The field was initially produced by waterflood since 1962. Starting in late 1966, oil in water emulsion was injected through three wells in a section of the field, as part of an effort to compare its efficiency with secondary recovery applied 
in a contiguous section, as showed in Fig. 2.16.

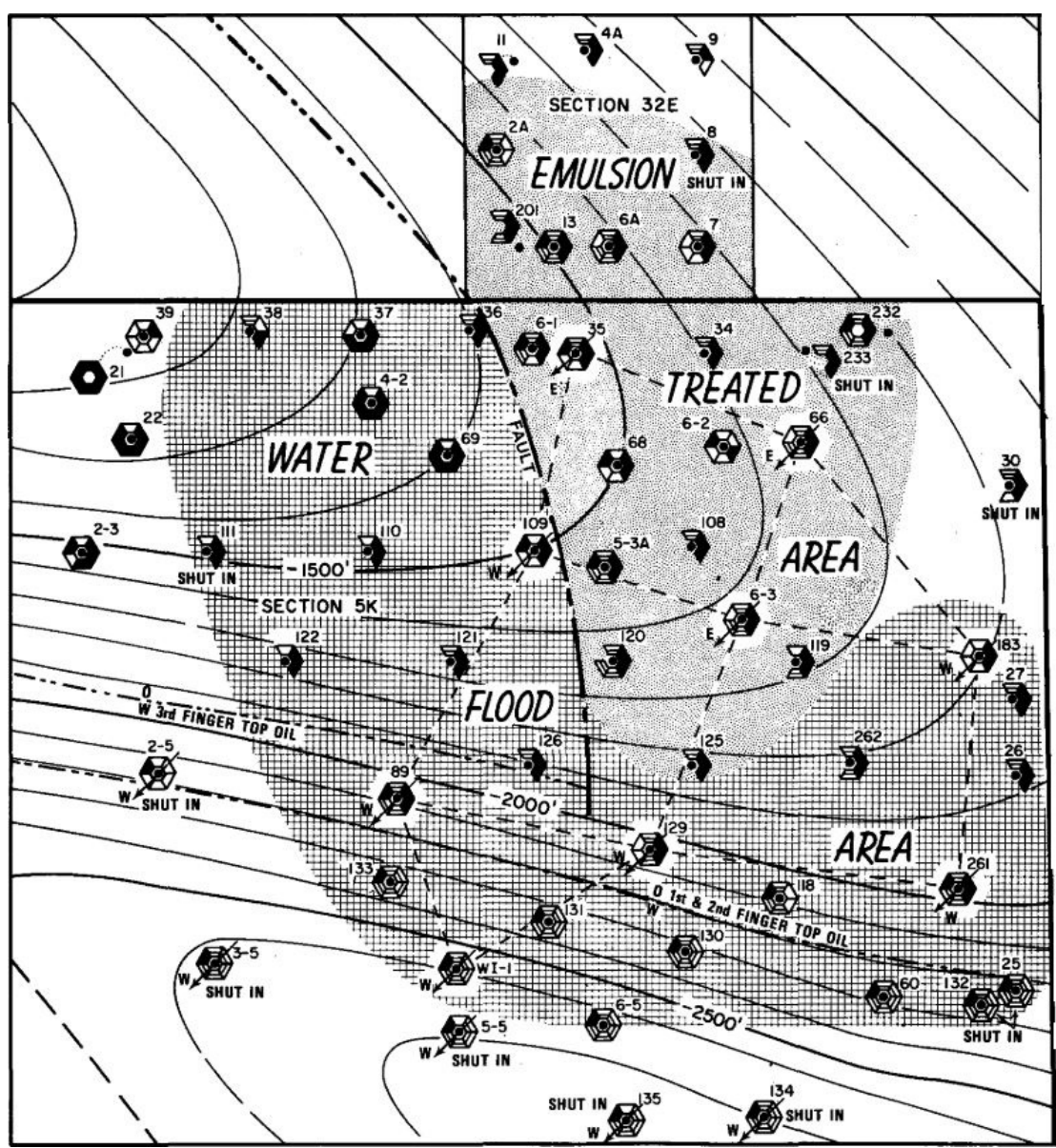

Figure 2.16: Adapted from McAuliffe [9]. Map of the Midway-Sunset field and well location.

The emulsion injected was mixed with water so that it comprised $14 \%$ of the total volume injected. After of 4 years, $3 \%$ PV (0.4\% PV of oil) were injected into the area believed to be affected by the emulsion. Figure 2.17 shows the results for the total fluid production, water-oil ratio, and oil production, from both the waterflood area and the emulsion swept area. It can be seen that both areas behaves in a similar way until the beginning of the emulsion treatment. After that, the waterflood area kept the decline rate of the oil production and increasing significantly the water-oil ratio. In contrast, the emulsion treated section presented an increase on oil production, keeping water-oil ratio on the levels prior to the treatment. 

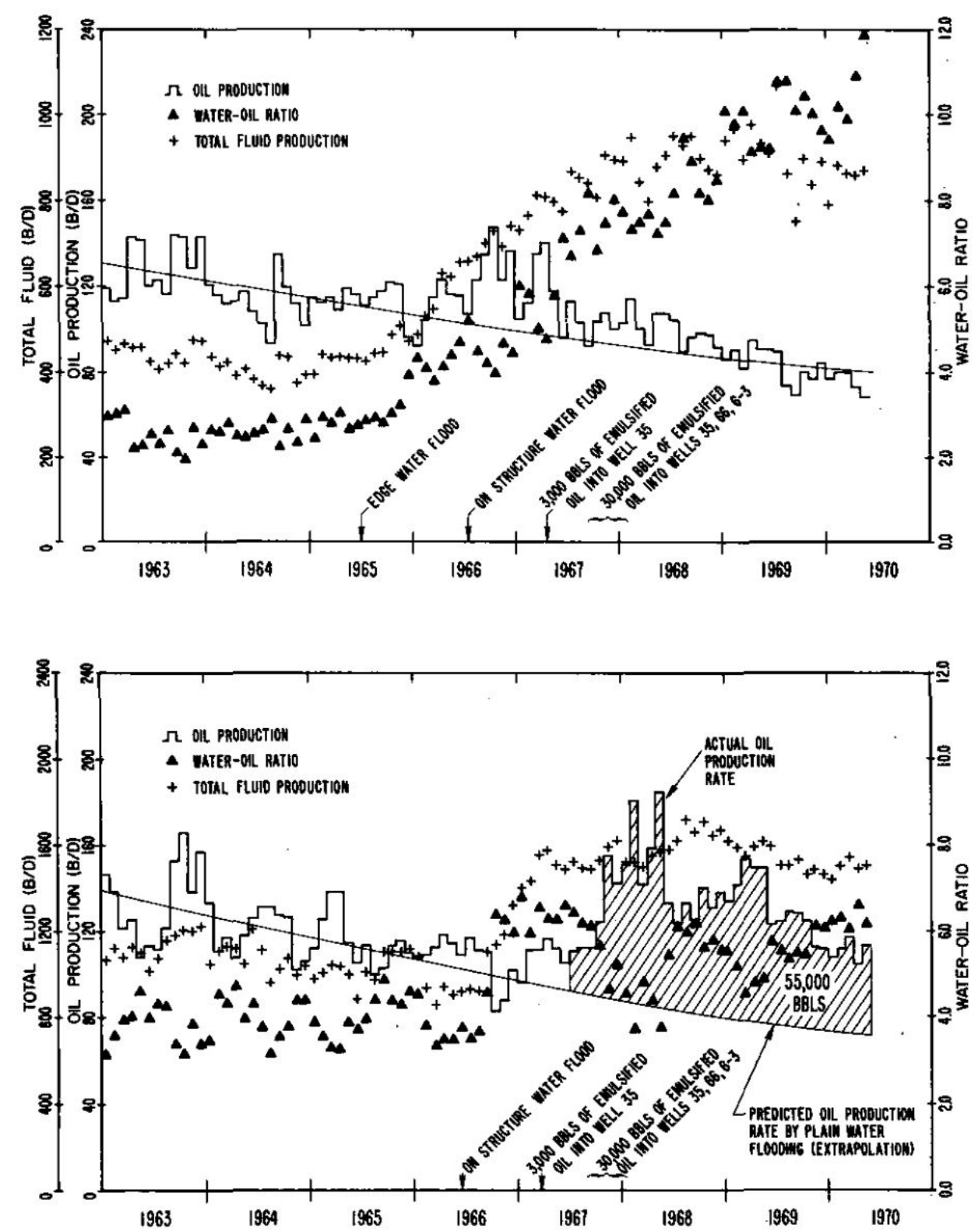

Figure 2.17: From McAuliffe [11]. On top, the fluid production from the waterflood area. At the bottom, the fluid production on the emulsion treated area.

McAuliffe concluded that the emulsion injection was operationally feasible and that its injection into the reservoir kept, and even increased the oil production, probably by controlling fingering and improving volumetric sweep efficiency. Apart from the increase in oil production and the control of wateroil ratio, these conclusions were supported also by the fact that there was an increase of the salinity of the produced water after emulsion injection (showing that the produced water was composed by less injected water than before). This was also suggested by radioactive tracer analysis.

Despite the advances made by these studies on emulsion injection as an 
EOR method, a known setback of this technique is the difficulty to obtain controlled mechanical properties (homogeneous diameters and stiffness) and homogeneous interfacial characteristics on the emulsion droplets. When these controlled mechanical properties are missed, worse is the performance of oil recovery. A possible way to address this problem is to use as blocking particles a special designed multiple-layered emulsion droplets, small prefabricated capsules, that can be precisely produced. This technique, known as microcapsules injection, will be discussed next.

\section{3}

\section{Microcapsules as a recovery method}

Multiple-layered emulsions are known for a long time in physicalchemistry and have many industrial applications. Such as on the release of drugs or nutrients in tissues [38], on encapsulation of fragrances [39] and even on the storage of carbon dioxide [40].

The use of capsules injection as an enhanced oil recovery method is an innovative technique that was inspired on the blocking properties of emulsion droplets formed or injected into the reservoir. Inside the porous medium the capsules behave in a similar way of the emulsion droplets blocking the water preferential path, increasing the pressure gradient and displacing oil left behind the by the waterflood. These capsules are also named microcapsules as their dimensions are in a micro scale, for example, in this work we tested a range of capsules with diameter between 100 to $200 \mu \mathrm{m}$.

For oil recovery, the most common approach is to work with microcapsules that are produced from double emulsions templates. The middle phase is solidified becoming a membrane or shell that confines the inner phase and maintains the capsule properties, even after changes on the outer phase.

One method to create double emulsion is by two steps emulsification. A simple emulsion is formed by shear stressing two immiscible fluids. Then this emulsion is mixed again (at a lower shear rate so not to break the droplets) with a third phase, immiscible with the continuous phase of the simple emulsion. Figure 2.18 present a diagram of how this method works. Although this method has the advantage of producing droplets in a large scale, this procedure is associated with a large diameter distribution and non uniform shell thickness of the double emulsion. This technique is commonly used on cosmetic and food industry, where the lack of control on diameter and shell thickness is not a critical matter. 


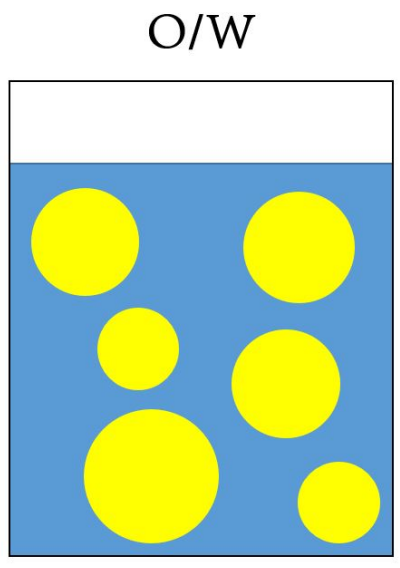

High shear

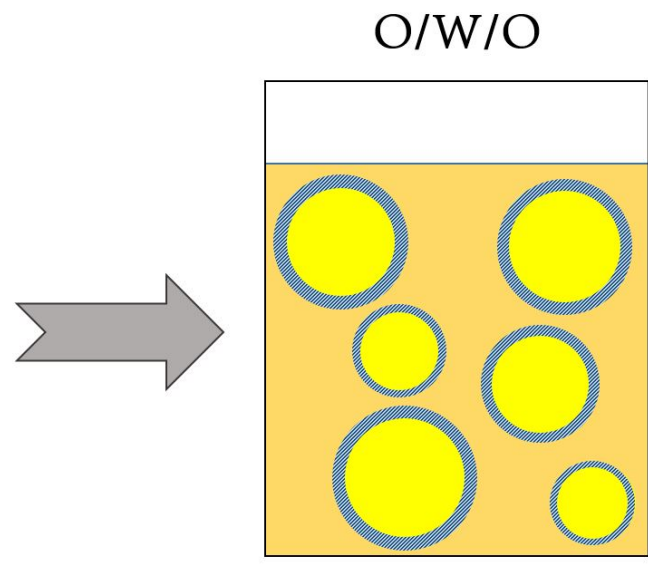

Low shear

Figure 2.18: Double step emulsion producing. In blue is represented the aqueous phase, in yellow and orange are represented the inner and outer oily phases, respectively.

Thus, to test the effect of capsules injection in a porous medium, another method of microcapsules production was needed. An usual method is to produce the capsules using microfluidic devices, that allows a precise formation of the droplet with high reproducibility. This method gives a better control of their mechanical properties: diameter, shell thickness and composition. The capsule stiffness is a function of these parameters.

Nascimento et al. [12], using a microfluidic method, tested microcapsules stabilized by polydimethylsiloxane (PDMS), investigating the effect of membrane thickness and polymer to cross-link ratio on the mechanical properties. The design of the device used is presented in Fig. 2.19.

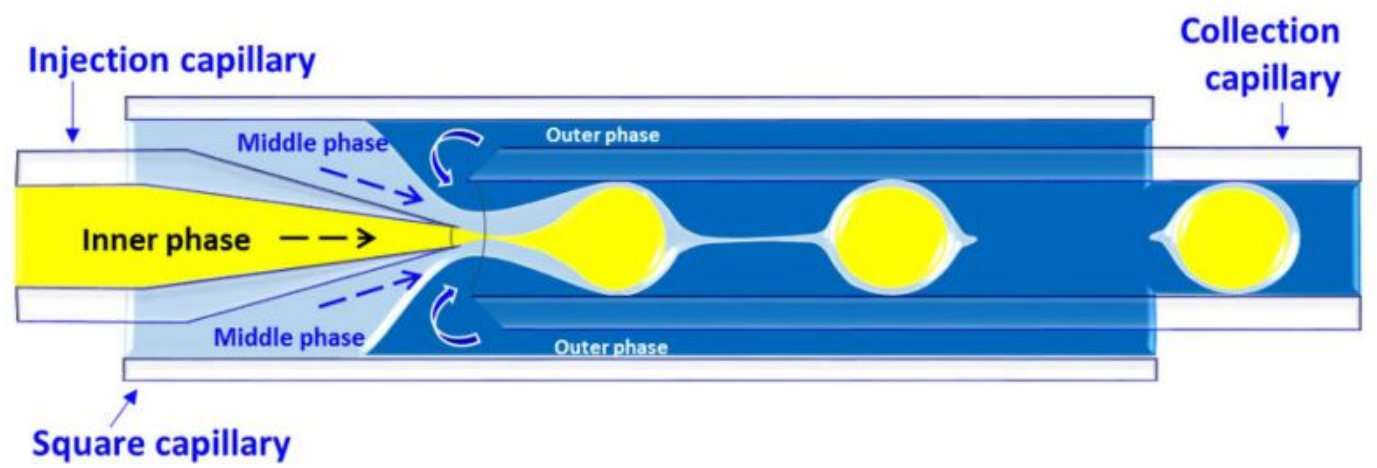

Figure 2.19: Illustration from Nascimento et al. [12]. Sketch of the microdevice for the capsule production.

Once the capsules were produced, Nascimento et al. tested the capsule apparent elastic modulus resulting from different shell thickness and composition in a rheometer, their results are shown in Fig. 2.20. Also in this work, 
the authors investigated experimentally the flow of microcapsules suspension through a constriction, producing images with a high velocity camera and measuring the pressure drop through the channel.

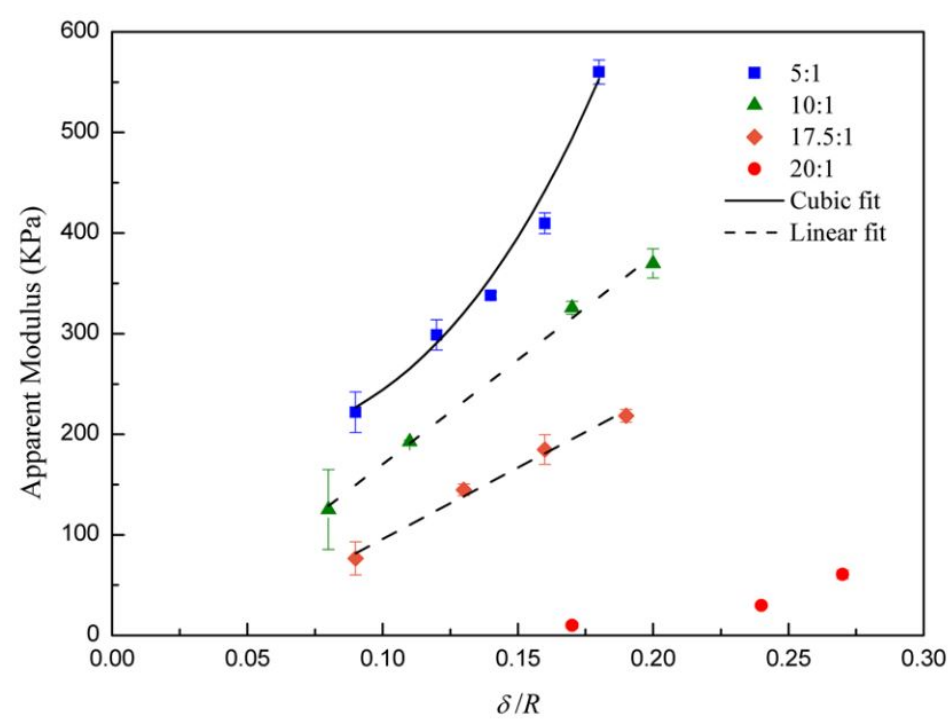

Figure 2.20: Result of the capsules elasticity from Nascimento et al. [12]. Plot of apparent modulus (maximum stress to maximum strain ratio) as function of shell thickness. Each color represent a different polymer to cross-link ratio.

Vimieiro [13] describes the application of the $\mathrm{W} / \mathrm{O} / \mathrm{W}$ microcapsules produced by Nascimento et al. as an alternative to emulsion injection on porous media for EOR. Capsules with specific polymer to cross-link ratio and diameter were injected at a fixed flow rate on a 3D porous device. The confocal microscopy technique was used to visualize oil recovery by aqueous phase injection and the microcapsules behavior. A visualization procedure was developed following the recommendations of Datta [20], whose work will be further discussed on the next section, which is dedicated to confocal microscopy.

The first part of the results obtained by Vimieiro [13] measured the amount of residual oil in the porous media after gradual increases on the water flow rate, in a water injection experiment. For each flow rate, the injection was kept until steady flow regime was reached and only then the 3D images were acquired. The result is an accordance to Payatakes [6]: the volume of residual oil decreases and the number of ganglia increases. This could be explained by the break up of large ganglia into smaller ones, as explained by Hinkley and is shown in Fig. 2.11.

Figure 2.21 shows the result of a typical experiment where the flow rate was increased on steps. Fluid inlet is on the bottom side of the sample. The image on the left, colored in green, is one of the 2D image slices that are used 
to reconstruct the 3D image. It shows the porous core saturated with water. The visualization on Vimieiro's work was based on the analysis of the aqueous phase. Thus, after segmentation, a binary image is formed where everything presented in white is the aqueous phase and the dark pixels are either oil or the glass beads that compose the porous medium. In Fig. 2.21, the images on the right of the green colored one show the status of a section of the core at different instants. From left to right these binary images show the section: saturated with the aqueous phase; saturated with the oil phase; after aqueous phase injection at $21 \mu \mathrm{l} / \mathrm{h}, 106 \mu \mathrm{l} / \mathrm{h}, 533 \mu \mathrm{l} / \mathrm{h}, 2.67 \mathrm{ml} / \mathrm{h}$ and $13.35 \mathrm{ml} / \mathrm{h}$, respectively. These flow rates correspond to values of $C a$ of $10^{-6}, 5 \times 10^{-6}$, $25 \times 10^{-6}, 125 \times 10^{-6}$ and $625 \times 10^{-6}$, respectively.

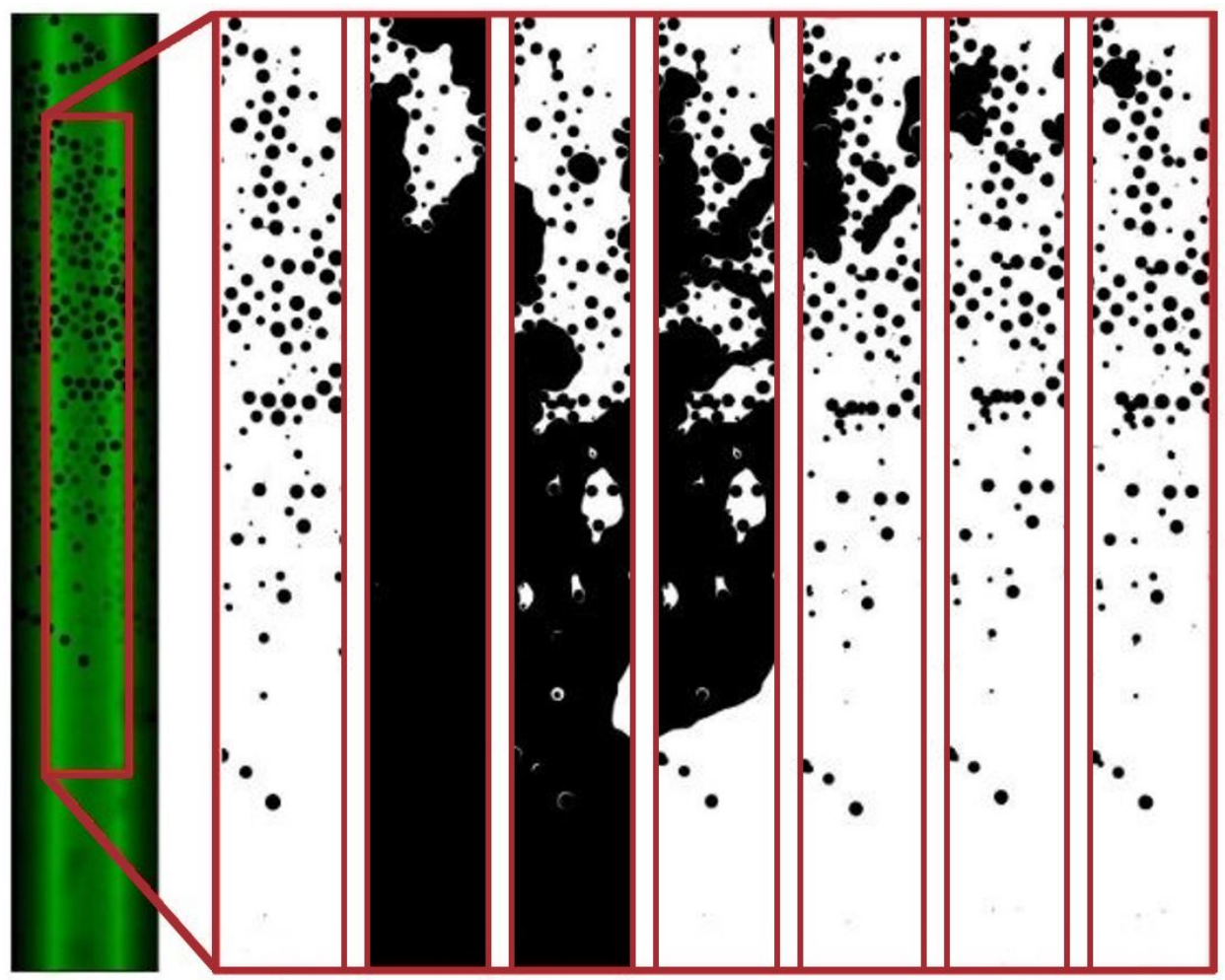

Figure 2.21: Adapted from Vimieiro [13]. Result of the gradual increase in water injection observed on one 2D slice of the porous core. Green in the first image and white in the others represents the aqueous phase. From left to right images show first the whole section of the core prior to image processing. Then, a fixed section of the core is shown: saturated with the aqueous phase; saturated with the oil phase; and after the aqueous phase injection at $21.355 \mu l / h, 106.755$ $\mu l / h, 533.775 \mu l / h, 2.67 \mathrm{ml} / \mathrm{h}$ and $13.35 \mathrm{ml} / \mathrm{h}$, respectively. Fluid inlet is at the bottom side of the core.

Vimieiro works with single phase segmentation, and the method is not able to distinguish the oil from the solid matrix. Thus, it is necessary to perform an image operation of subtraction between the image of a section saturated with the aqueous phase and the image obtained after each flow rate to discover 
the residual amount of oil. Figure 2.22 shows an example of the subtraction operation done, where "I" is the image of the section saturated with water, "II" is the image of the section after running water injection at a given flow rate and "III" is the subtraction "I"-"II". Notice that when this image subtraction is performed, the resulting image ("III") shows in white only the remaining oil and the water and glass spheres appear in black.
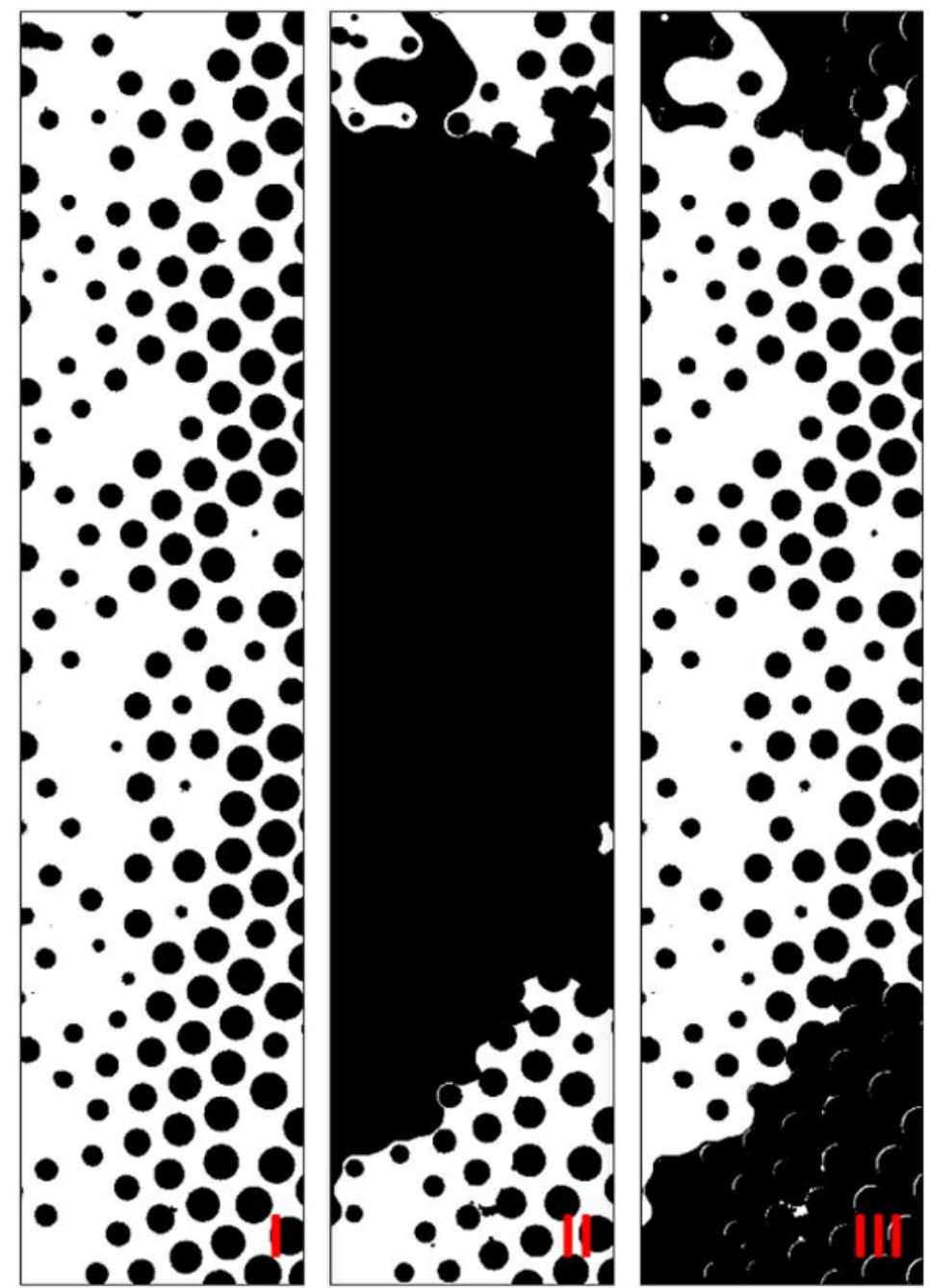

Figure 2.22: Subtraction operation done by Vimieiro [13] to discover the amount of remaining oil in each image. "I" is the section saturated with water, "II" is the section after running the desired flow rate and "III" is the subtraction "I"-"II". In "I" and "II" water is presented on white and the oil and the porous core in black. In "III", oil is in white and water and the porous core are presented in black.

The same experiment presented in Fig. 2.21 was repeated for other porous devices. These devices were built with the same materials and were supposed to be a replica of the first one. After image processing, it was possible to quantify the amount of residual oil in each case and compare them. The results of these experiments are presented in Fig. 2.23, showing the residual oil saturation for 
the different devices as a function of the Capillary Number. A considerable variation of the results was observed.

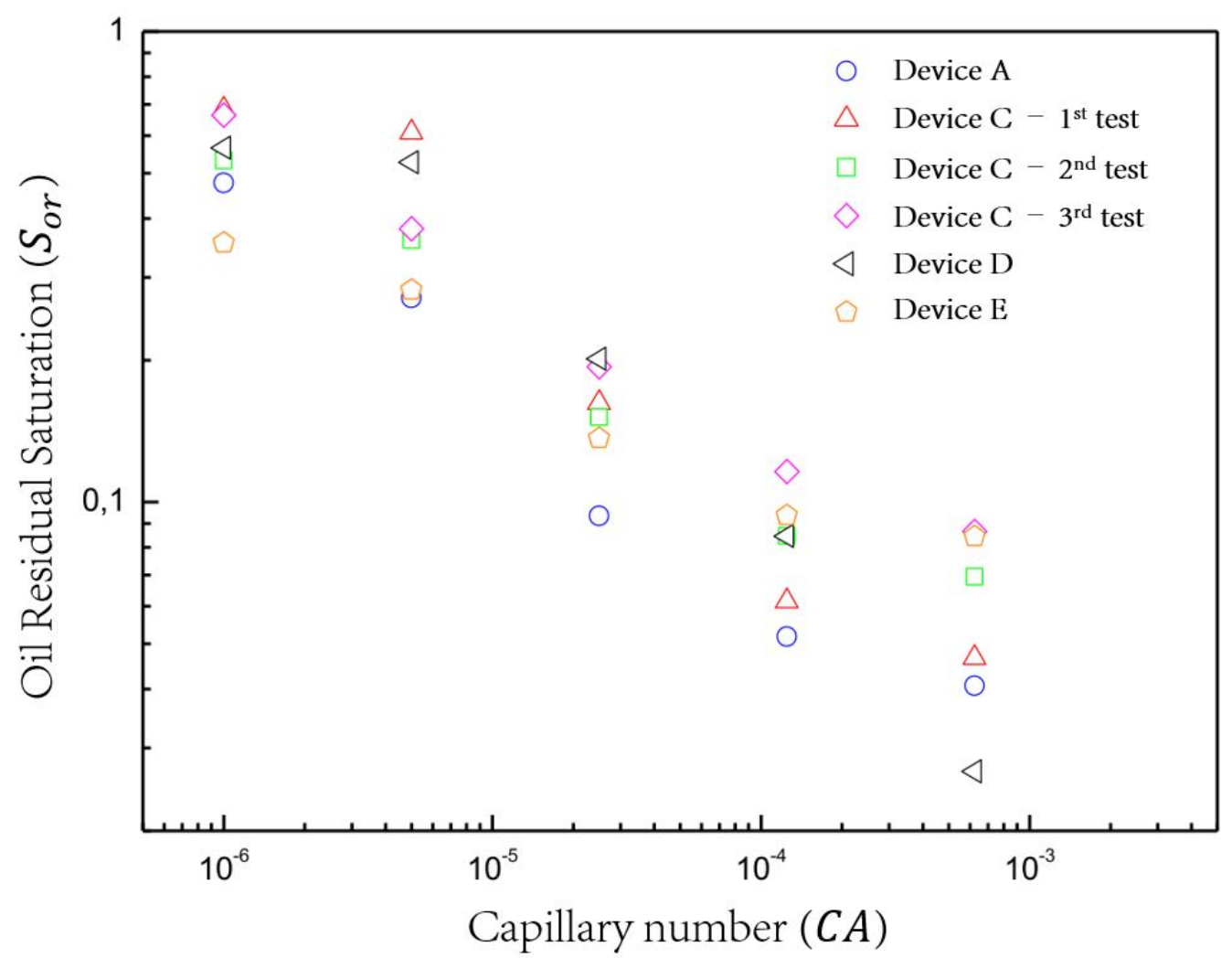

Figure 2.23: Oil residual saturation for different devices as a function of the Capillary Number, from Vimieiro [13].

After the results with the aqueous phase injection, Vimieiro calculated the porous channel average size using two methods, so to design the size of the PDMS microcapsules to be injected. The average diameter of $200 \mu \mathrm{m}$ was chosen for a predicted porous channel average size of $100 \mu \mathrm{m}$.

The first three experiments with microcapsules injection resulted in a small increase in the oil recovery efficiency, an average of $4 \%$. Additionally, an interesting qualitative result came from the image analysis, showing ganglia mobilization and break up. On the other hand, Vimieiro result's show that the microcapsules did not penetrate very far in the porous medium. So, a second set of experiments was performed, this time using microcapsules with the same diameter but with higher cross-link ratio, making capsules with lower stiffness. The result of the second set of experiments were quite similar to the first one and the capsules did not penetrate much further into the device.

In the conclusions, Vimieiro claims that microcapsules injection seems to improve oil recovery. However, the observed behavior of the capsules not penetrating deep into the porous medium indicates that further work was 
needed to explore the physics of oil recovery by injection of a suspension of microcapsules.

Trying to reach more precise and adequate values for capsules diameter and stiffness, different methods for fabricating the microcapsules were proposed. In order to discover a way to produce a softer type of microcapsule, Michelon, Leopércio and Carvalho [14] performed a survey on variations of Nascimento et al. device's, testing different capillary shapes and dimensions, fluids, materials and wettability conditions.

Their work was of major importance to the development of this thesis, where microcapsules produced according to the method described in their paper were used. These were $\mathrm{O} / \mathrm{W} / \mathrm{O}$ double emulsion droplets with hydrogelbased shells, fabricated with glass-capillary microfluidic devices. The 3D microfluidic devices were designed with a specific geometry and using proper flow rates, so as to stabilize the capsules formation.

Figure 2.24 shows that the devices were built using two treated capillaries inserted into a small square tube. It was important that the two capillaries were perfectly aligned. Michelon et al.'s work describes two types (designs, as they call) of microfluidic devices, each one for the production of capsules with specific properties. The parameters that differ them are the inner diameter of the capillaries, $d_{i}$ and $d_{c}$, and the distance between them, $l$. Apart from the geometry, the mechanical properties will depend on each phase flow rate, named as $Q_{i}, Q_{m}$ and $Q_{o}$, the flow rate of the inner, middle and continuous phases, respectively. 


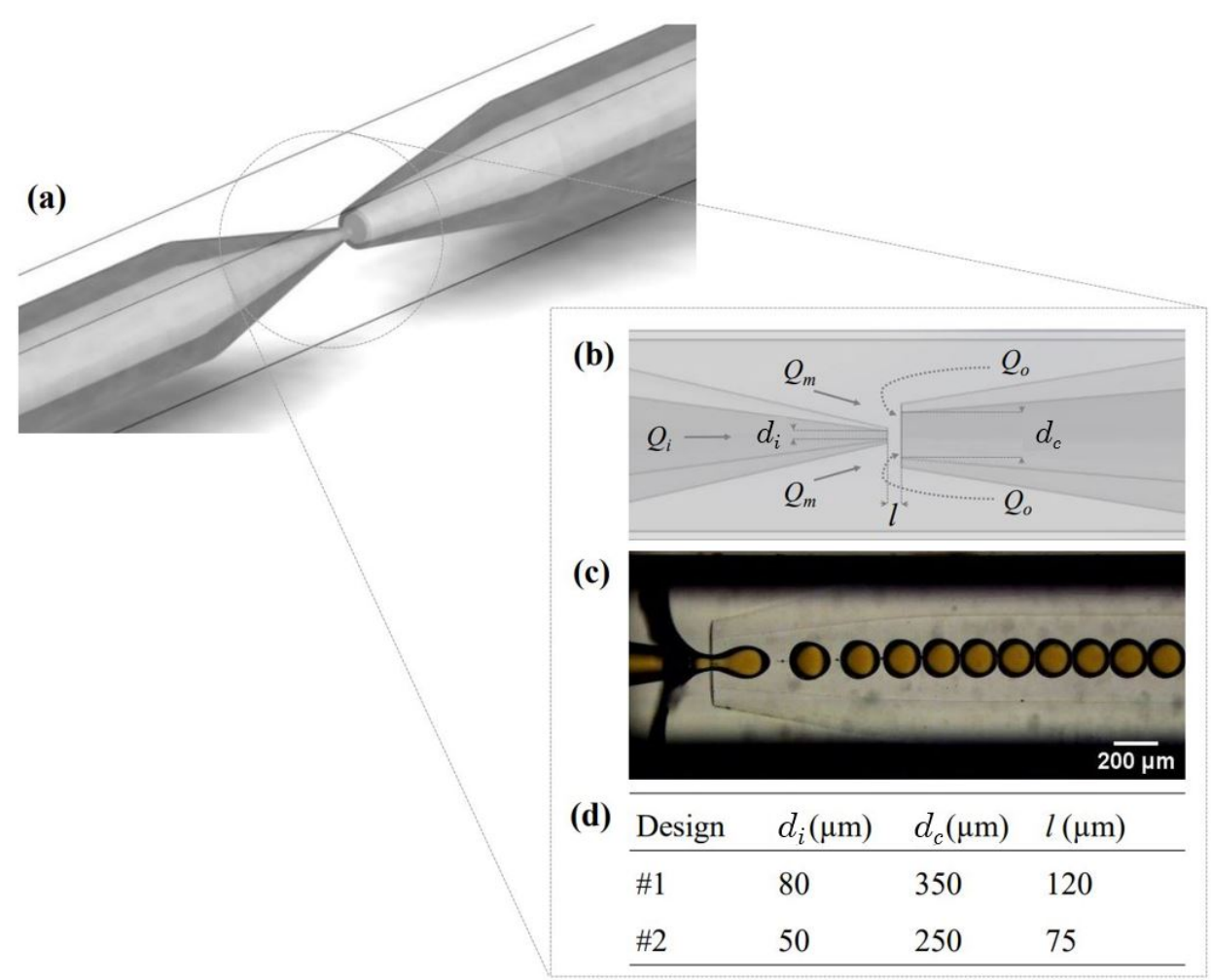

Figure 2.24: Adapted from Michelon et al. [14]. (a) shows a perspective view from a model of the glass capillary device; (b) shows a diagram of the region of droplet formation. $Q_{i}, Q_{m}$ and $Q_{o}$ are the flow rates of inner, middle and continuous phases, respectively; (c) shows a picture of capsules being formed; and (d) informs the geometric parameters of the devices used. $d_{i}$ and $d_{c}$ are the inner diameter of the injection and collection capillary tubes, respectively, and $l$ denotes the separation distance between them.

The inner phase used by Michelon et al. was a sunflower oil with an orange food-grade dye. The middle phase composition was a mixture of 0.5 wt.\% lowacyl gellan gum and 2 wt.\% polyoxyethylene sorbitan monolaurate, Tween ${ }^{\circledR}$ 20 , in ultrapure water. The continuous phase was a sunflower oil dispersion containing 1 wt.\% calcium acetate and 5 wt.\% polyglycerol-polyricinoleate. Each phase density and viscosity was measured as well as the interfacial tensions between the middle and the inner phase and the middle and the continuous phase. The microcapsules were collected in glass vials filled with hexane. After that, a small amount of acetate buffer was put inside the vial in order to separate the microcapsules from the hexane and the continuous phase for later applications.

Following the microcapsules production procedure described by Michelon et al. [14], several capsule mechanical properties were obtained for use in this thesis experiments. Eventually, it was decided to use here microcapsules with $104 \mu \mathrm{m}$ of diameter and $5 \mu \mathrm{m}$ of shell thickness. This point will be explained in details in Experimental Procedure chapter. 


\section{4}

\section{Confocal laser scanning microscopy}

This section covers the confocal laser scanning microscopy (CLSM) technique physics, describes some of its usual applications and reviews the first work using this technique on microfluidic experiments. A review on this technique can be found in the work of Semwogerere and Weeks [15] and in Hepler and Gunning [41].

Usual microscopes are not really convenient for studying phenomena happening in 3D structures because the optics tools used consist of a superposition of in-focus and out-of-focus regions. The point of the confocal microscopy technique is to obtain sharp images that would appear blurred when using normal microscopes. Figure 2.25, from [15], shows the difference between images from a normal microscope and from a confocal microscope.

A

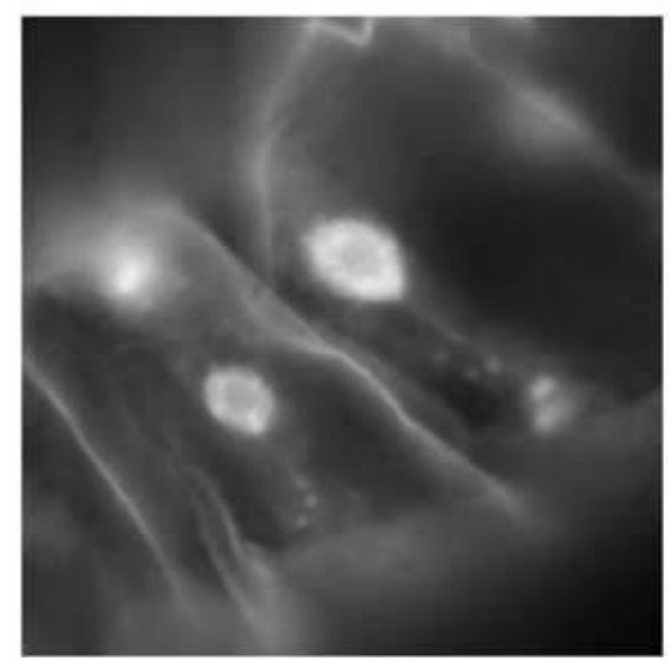

B

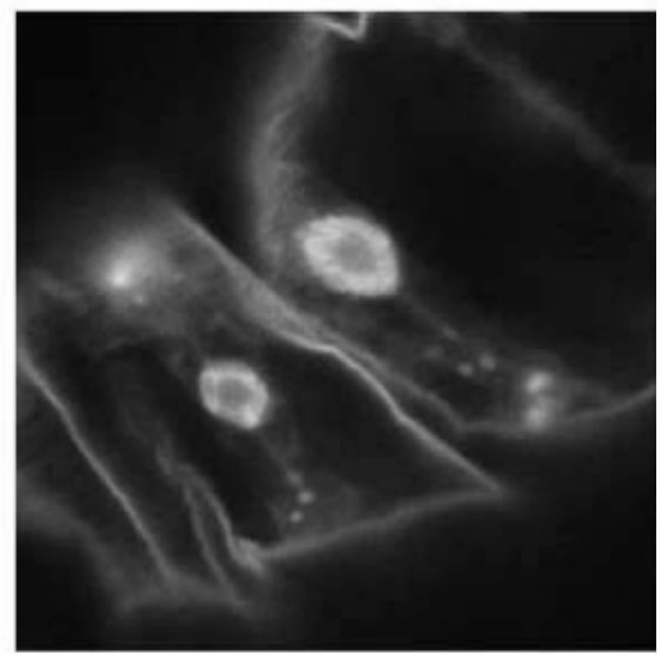

Figure 2.25: Image from Semwogerere and Weeks [15]. Images of cells of spirogyra generated by (A) normal microscope and (B) confocal microscope .

Furthermore, by obtaining many clear 2D images, varying the focal plane, it is possible to reconstruct a 3D specimen by stacking these thin image slices. This can be seen in Fig. 2.26, adapted from Claxton et al. [16], where a pollen grain is shown in different planes and in its 3D reconstruction. 

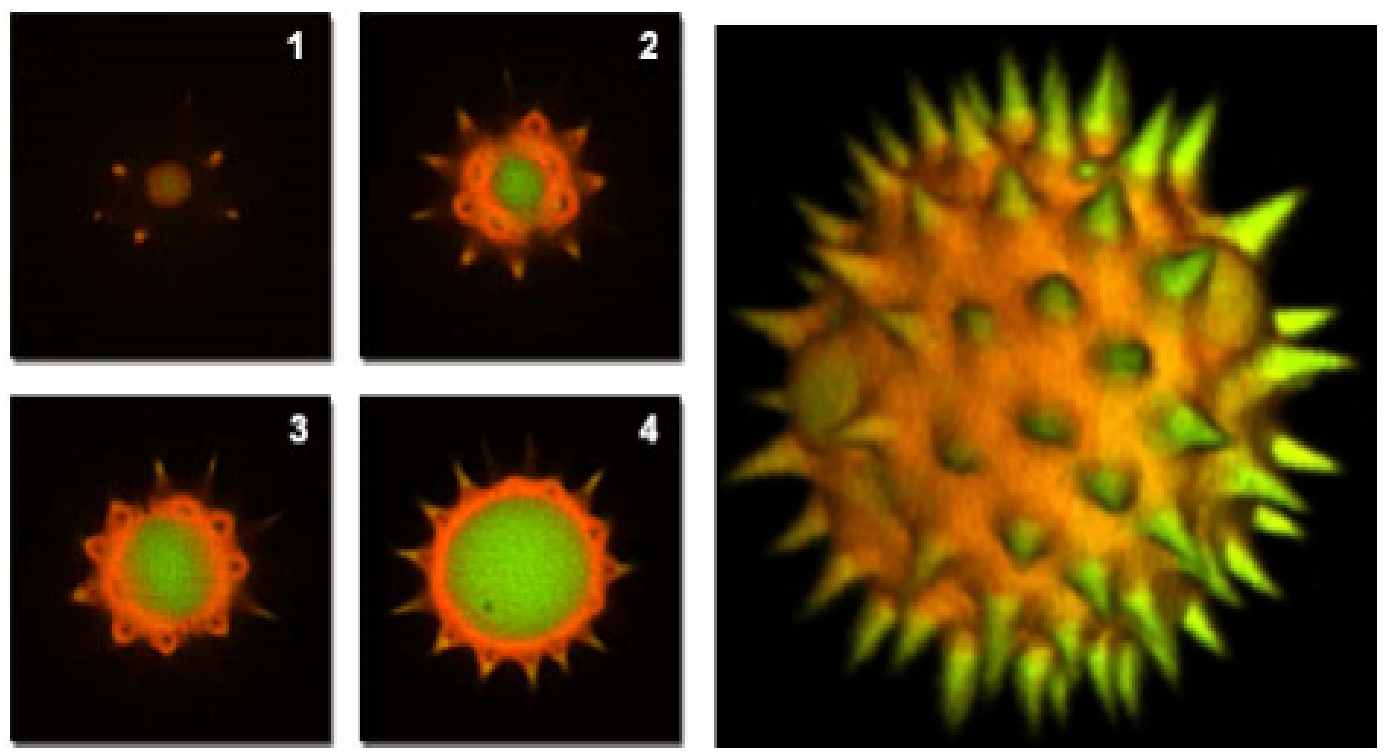

Figure 2.26: Different focal planes of a pollen grain visualized on the confocal microscope are shown numbered on the left side and a 3D reconstruction many thin slices is presented on the right side of the figure. Adapted from Claxton et al. [16].

The desired 2D image is the result of optical tools that exclude most out of focus light from the image formed by the microscope. The optical tool that allows the focused-only image capture is the pinhole, illustrated in Fig. 2.27 by Borlinghaus [17].
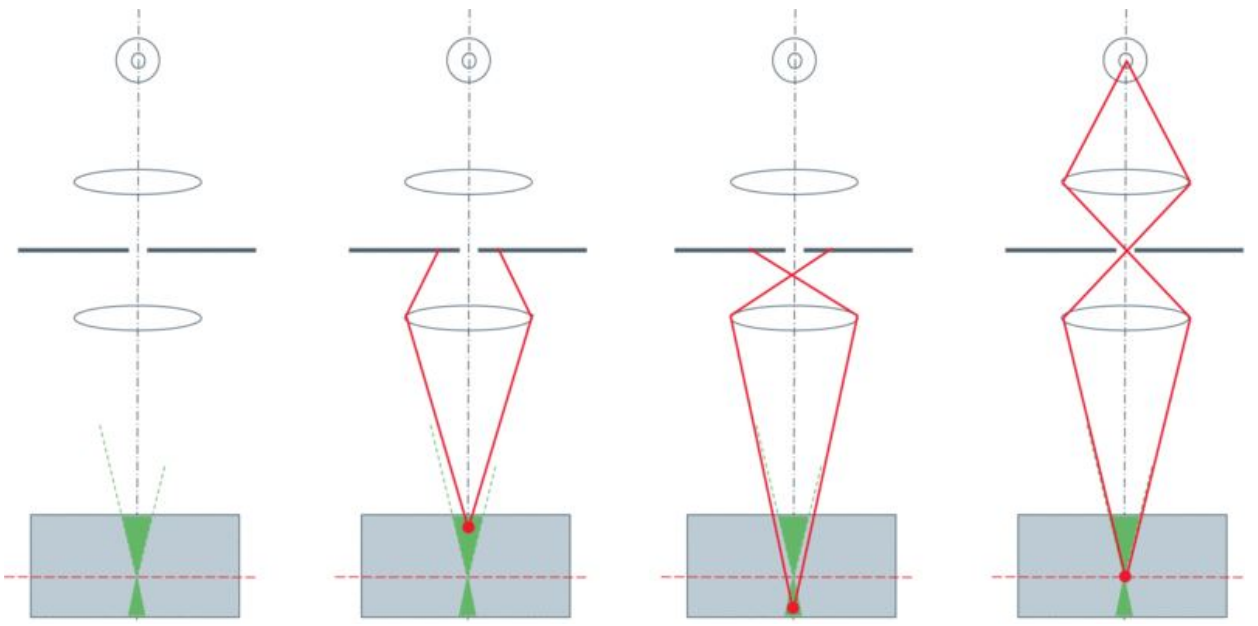

Figure 2.27: Illustration of the concept of the pinhole tool from Borlinghaus [17]. The laser (in green) excites the sample not only in the focal plane, but also above and below. Light emitted from above and below the focal plane will not pass the pinhole. Only light that originates from the focal plane will reach the detector.

But, in order to capture images in planes deep into the object, it is important to have the whole sample transparent and with the same refractive 
index. However, this two optical conditions alone would produce an image in the microscope without any phase distinction. Thus, to visualize the different phases, each one has to emit light at a separate wavelength, this is achieved by using natural or induced (by pigmentation) fluorescence.

The physics of fluorescence is explained in Fig. 2.28, from Semwogerere and Weeks [15]. The molecules, at normal conditions, stay at their lower energy state (ground state). Once these molecules are excited by a laser and absorb a photon of light, they increase their energy and jump to a higher excited state. After reaching that state, the molecule quickly dissipates a part of the energy through collisions with surrounding molecules, causing the electron to drop to a lower energy level. The molecules at this lower excitation state will have then a light emission at a longer wavelength.

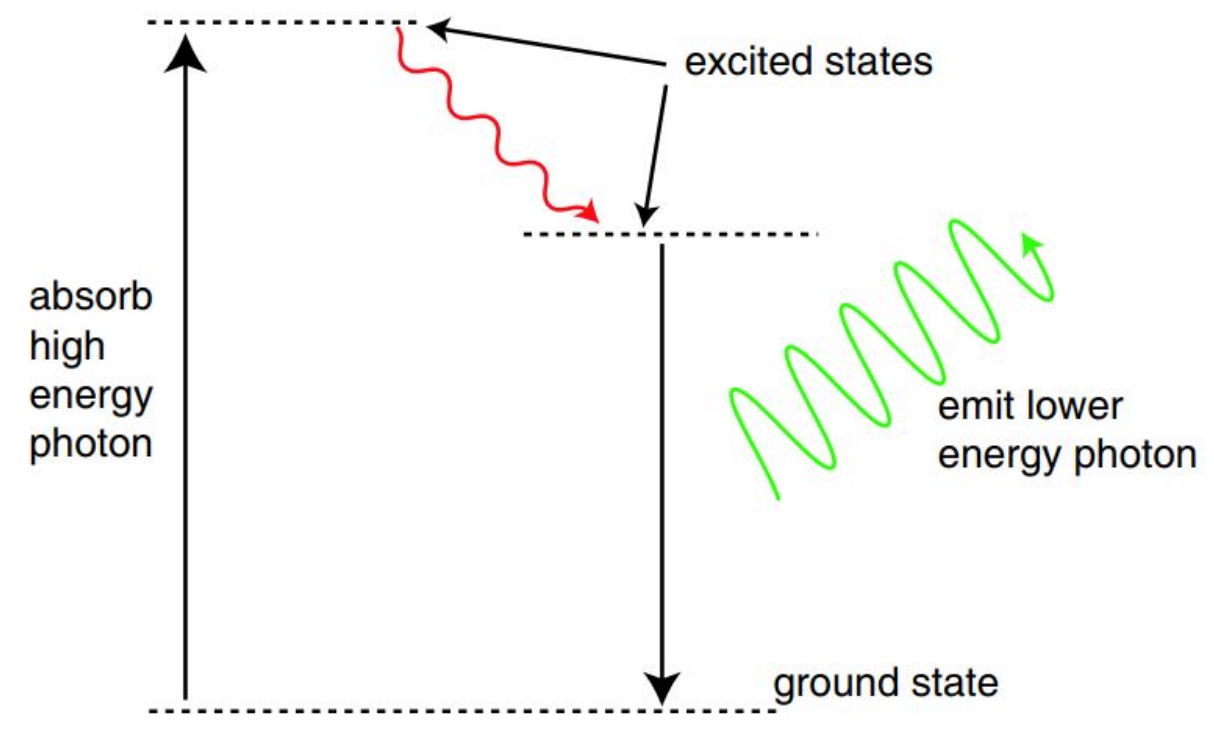

Figure 2.28: Illustration of fluorescence emission from Semwogerere and Weeks [15]. Molecules absorbs photon energy up to a higher energy state, loses a little of that energy to other molecules and then emit the rest emitting light of a lower energy.

Using this fluorescent technique on a sample with different fluorophores allows to visualize simultaneously multiple phases, each one emitting light at a different energy. Thus, by exciting the sample with different laser frequencies and capturing the response with the proper frequency detector, each phase of the specimen can be distinguished at the same time.

Also the confocal microscope uses laser scanning technique, that is meant to concentrate the laser light around the focal plane. With this, one can get better illumination of the neighborhood of the sample that is being captured by the pinhole. To do this, it makes use of rotational mirrors to direct the 
laser beans on the sample, promoting a precise and punctual illumination and excitation. This enhances the quality of resulting images.

CLSM is widely applied in Biological Sciences. Hepler and Gunning [41] provide a review of applications on imaging of plant cells. Pawley [42] provides a more detailed report on applications of CLSM to all areas of Biology. Another field where confocal microscopy has been applied lately is Material Sciences, [18]. Figure 2.29 shows a comparison done by Hovis and Heuer where the confocal image is noticeably sharper, even with almost the same resolution.
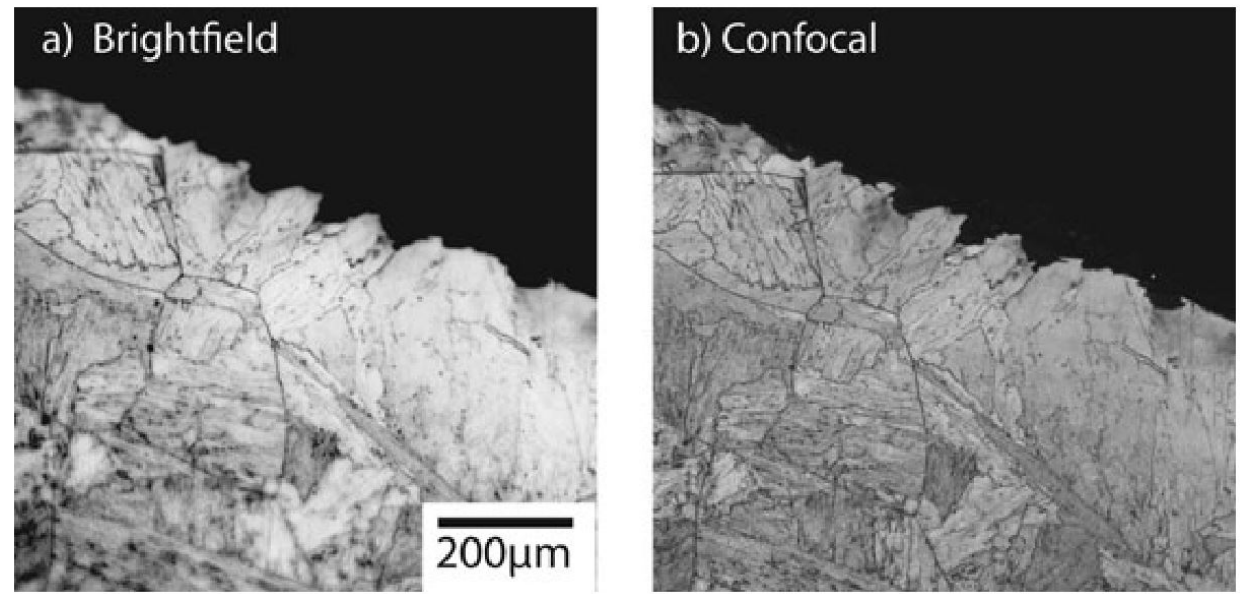

Figure 2.29: Images obtained by Hovis and Heuer [18]. (a) Conventional bright field image of a martensitic stainless steel sample. (b) An image of the same region taken using CLSM technique.

\subsection{1}

\section{CLSM technique applied to porous media flow}

It seems that the pioneer application of CLSM to microfluidics is found in a series of publications produced in Harvard. In the first work by Krummel et al. [19], it is described the fabrication of a porous medium in which a experimental procedure of 3D visualization with confocal microscope was performed. They calculated the porosity by visualizing different regions of the porous medium. After that, their work focused on the drainage, displacement of wetting fluid (aqueous phase) by a non-wetting fluid (oil phase), and on the imbibition, displacement of the non-wetting fluid by the wetting fluid.

Krummel et al. varied the Capillary Number for both the drainage and the imbibition experiment. The device was composed with glass beads with average radius of $75 \mu \mathrm{m}$ and its cross section had $3 \mathrm{~mm}$ width. However, images were obtained only a single optical slice of $11 \mu \mathrm{m}$ thickness.

Results for the drainage experiment are shown in Fig. 2.30. For both Ca shown in Fig. 2.30, the leftmost picture shows the initial state of the 
porous medium saturated with wetting fluid and a oil ganglion. In the other images, numerical subtraction was performed so to show the evolution of the oil occupying the porous medium. It can be seen that the phenomenon depends on $C a$.
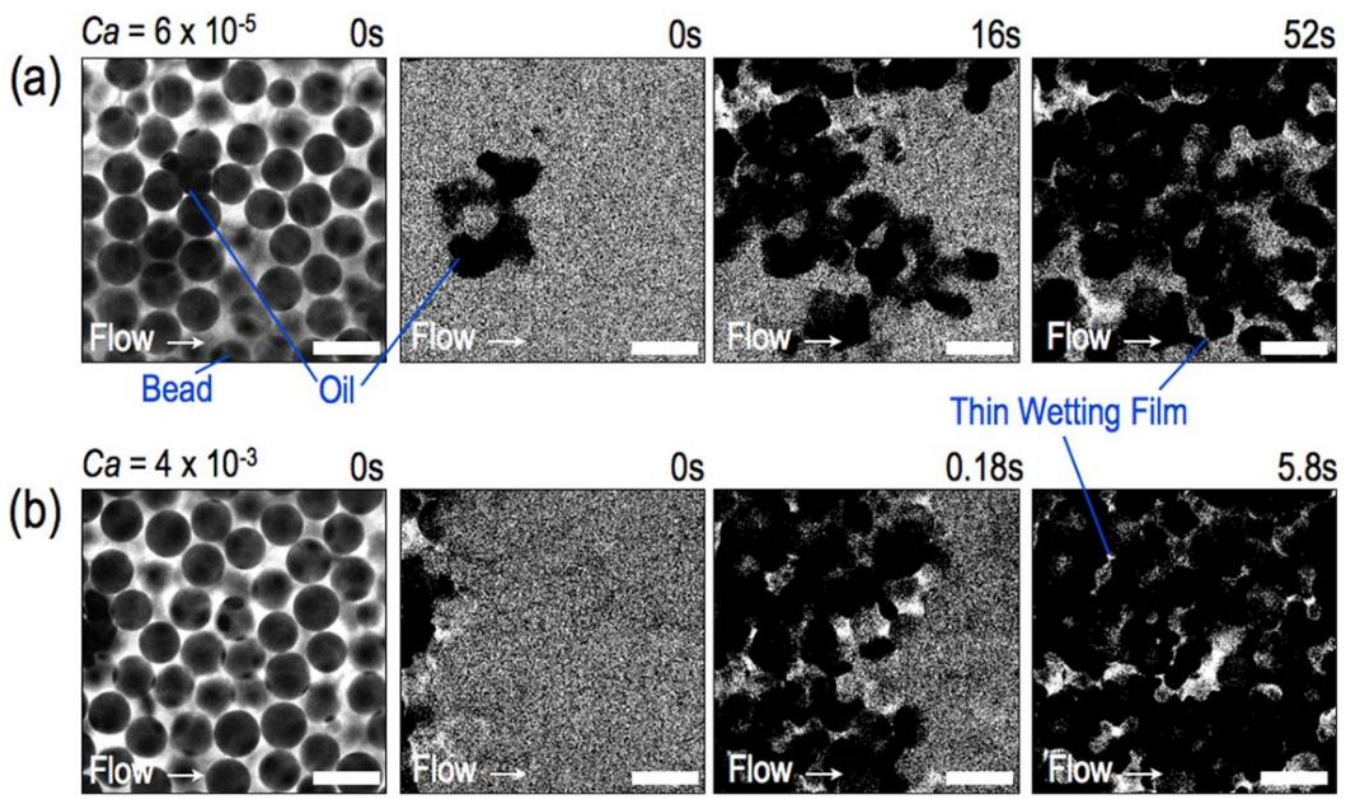

Os

Thin Wetting Film

Figure 2.30: Experiment performed by Krummel et al. [19]. Pictures on the left show the porous medium saturated with aqueous (wetting) phase and an oil ganglion. In the following pictures, showing the time evolution of the drainage, numerical image processing was performed so to show only the invading phase. The drainage efficiency clearly depends on $C a$.

A similar experiment was performed for imbibition, and the results are presented on Fig. 2.31. This time, the image subtraction was not performed and one can see the evolution of the imbibition of the aqueous phase. It seems that the imbibition has a stronger dependency on $C a$ than drainage. 


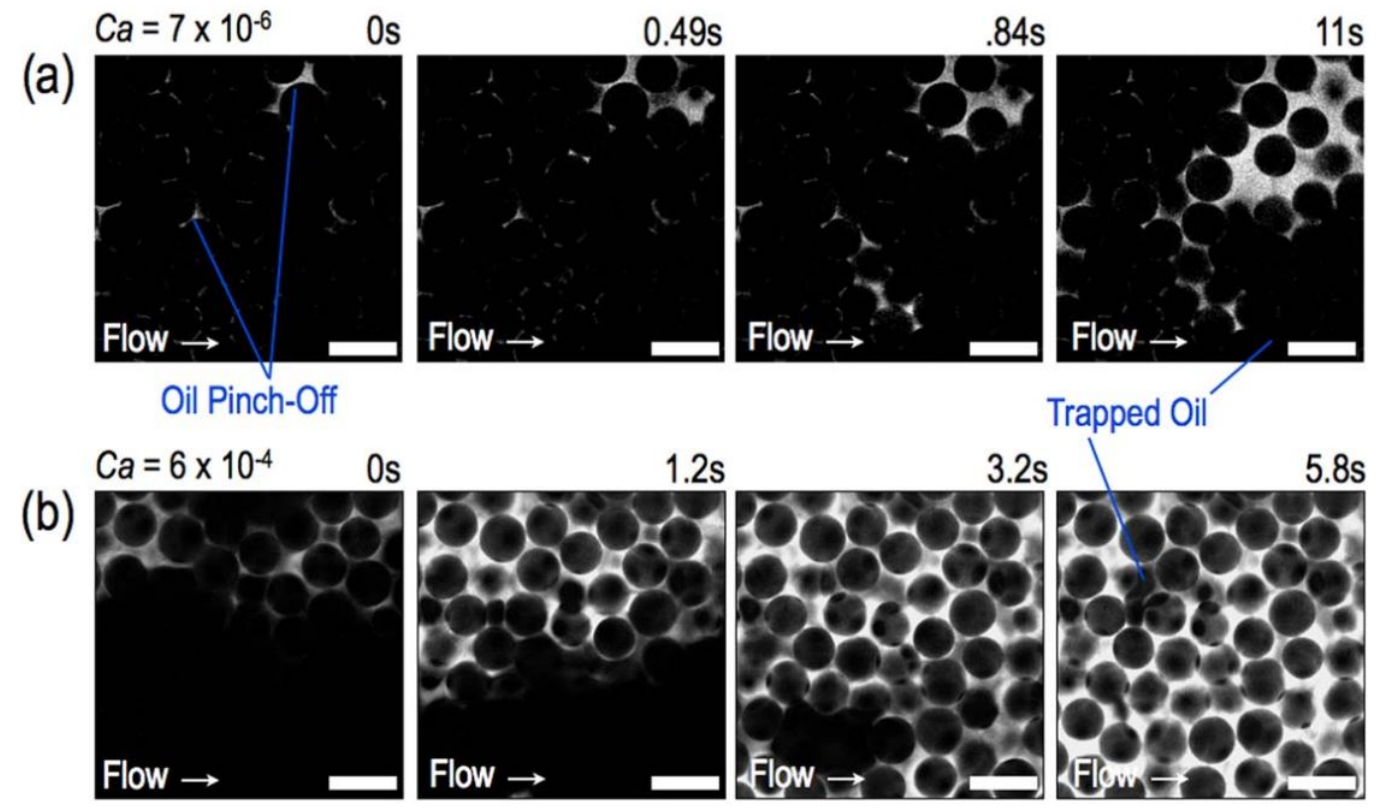

Figure 2.31: Imbibition experiment performed by Krummel et al. [19]. Sequences show the aqueous (wetting) phase displacing oil along the time. Imbibition efficiency highly depends on $C a$.

Krummel et al. also produced 3D pictures (by stacking thin slices) of the oil ganglia that remain trapped after imbibition, shown in Fig. 2.32. For the smaller value of $C a$, ganglia are ramified and span many pores spaces. Higher values of $\mathrm{Ca}$ produces smaller and less ramified ganglia. Based on the images, by quantifying voxels, the authors measure the residual oil saturation for different $C a$.

(a)

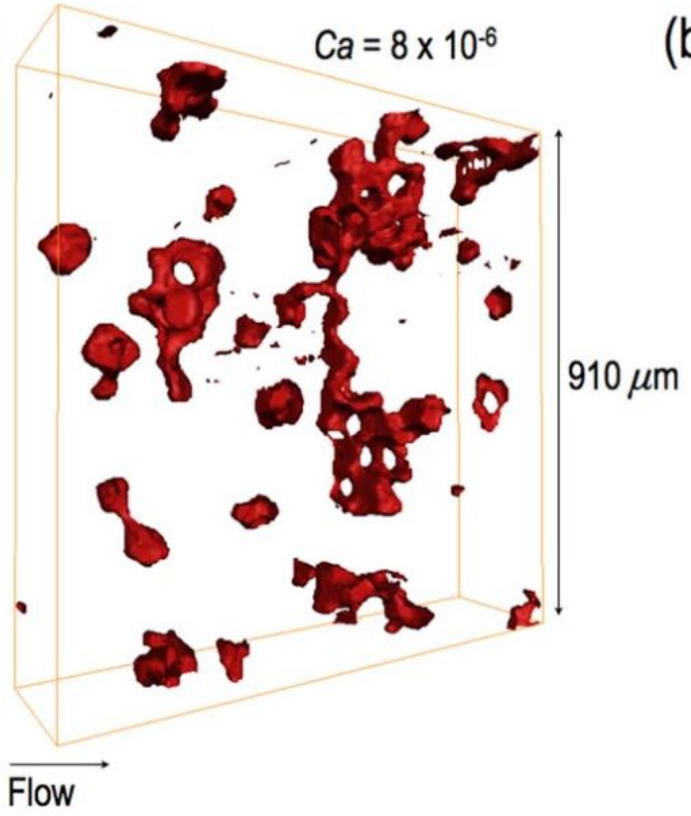

(b)

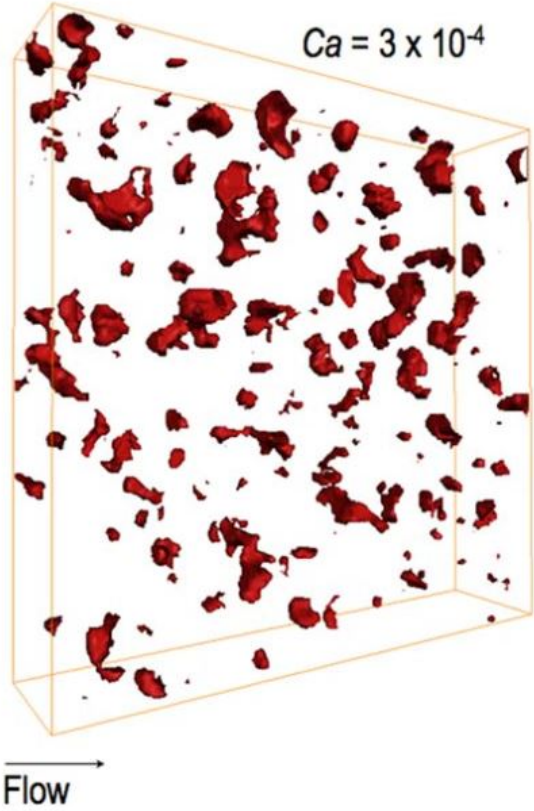

Figure 2.32: 3D images showing ganglia left in the porous device after imbibition at different values for $C a$. Image from Krummel et al. [19]. 
Datta and Weitz [20] build upon Krummel et al. methodology and present a detailed analysis of the drainage process in stratified porous media. The devices were built using spheres of different diameters so that the porous media was composed by coarse, fine and, in some cases, intermediate strata. Figure 2.33 shows a schematic view of the device and the separation of the glass beads inside the porous medium.

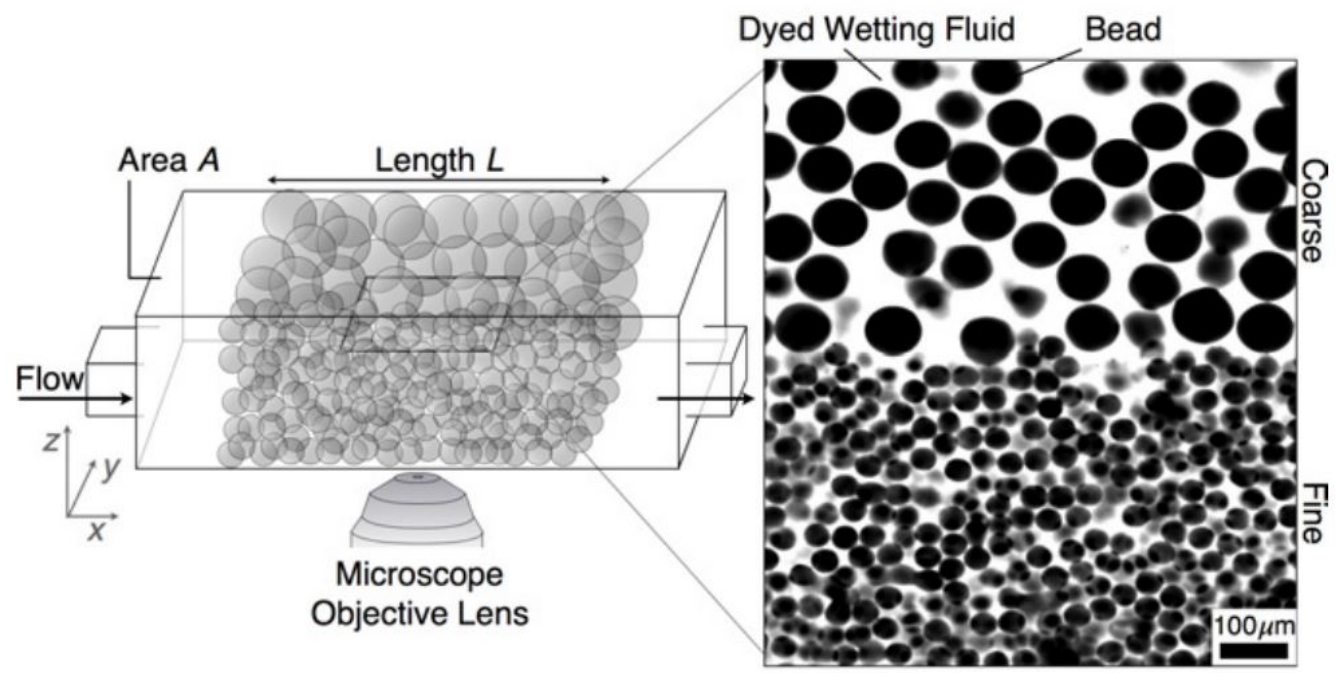

Figure 2.33: Schematic view of one of the stratified devices, showing a clear division between the coarse and fine strata. Image from Datta and Weitz [20].

Figure 2.34 shows a typical result of drainage by the oil phase for increasing values of $C a$. As one can expect, drainage at low flow rates is concentrated in the coarse strata. By increasing $C a$, the oil phase gradually invades the fine strata, according to the flow direction (from left to right). 


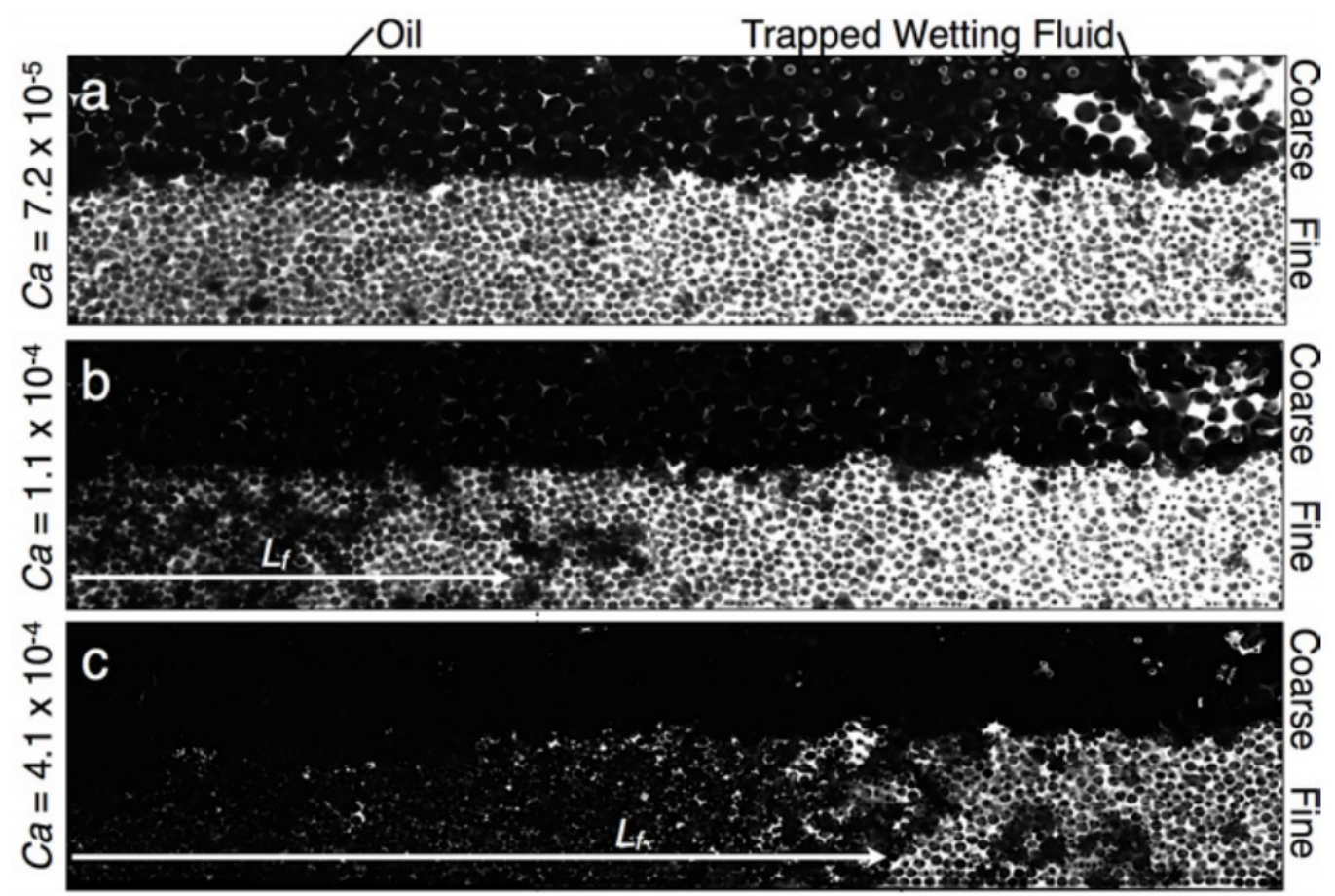

Figure 2.34: Drainage by oil in a stratified porous medium for increasing values of $\mathrm{Ca}$. Image adapted from Datta and Weitz [20].

Later, Datta et al. [5] using a porous device with uniform glass beads (38 $\mu \mathrm{m}$ diameter) presented results using CLSM visualization of (1) a complete drainage with oil (non-wetting) up to the connate aqueous (wetting) phase saturation; followed by (2) oil displacement in an imbibition experiment. Figure 2.35(a) shows the schematic of the porous device. Figure 2.35(b) shows the imbibition front invading the porous medium from left to right. The clear spots are the aqueous phase, and one can see connate water to the right of the picture. A fixed region of one slice of the porous device is shown in Fig. 2.35(c)-(d) for different times during the imbibition. 


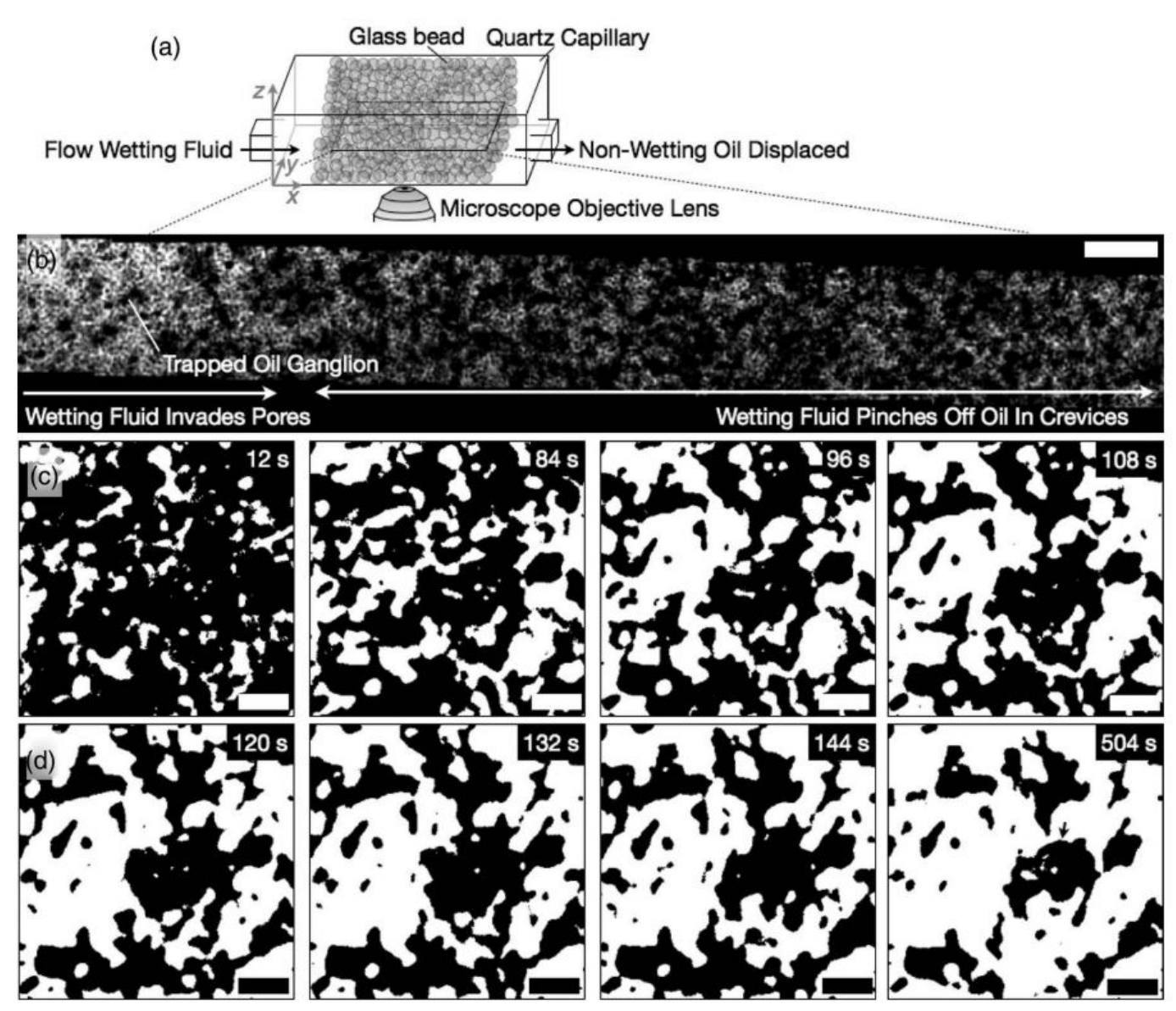

Figure 2.35: Image from Datta et al. [5], showing the porous device (a); the imbibition front invading from left to right (b); and one slice of a region in the porous medium in different times along the imbibition process.

Compared to previous works of their research group, Datta et al. [5] were able to advance regarding the quantification of the phenomenon based on visual information. They manage to measure the decrease of the residual oil saturation for increasing values of $\mathrm{Ca}$. They also characterized the distribution of ganglia lengths along the flow direction as a function of $C a$, quantifying the phenomenon observed in Fig. 2.32; and measured the maximum ganglion length as a function of $C a$. Finally, they were able to calculate the relative permeability curve for the wetting fluid as a function of its saturation combining visual information and pressure transducers measurements.

Other work from Datta et al. [43] combined dynamic CLSM visualization with PIV (Particle Image Velocimetry) techniques to characterize the velocity field in the porous channels, in both along and transverse components to the imposed flow direction. They found that the magnitude of the velocity in the transverse direction showed an exponential Probability Density Function (PDF). This fact implies that fluid flow in pore space is not completely random, otherwise the PDF would be a Gaussian. This is a surprising result, because 
considering the complexity of the porous spaces and the heterogeneity imposed by the presence of two fluids, flow through it is not completely random.

Datta, Dupin and Weitz [44] designed an experiment to investigate ganglia break up during two phase flow through a uniform porous medium composed by glass beads, using CLSM. To do so, they inject simultaneously both the oil (non-wetting) phase and the aqueous (wetting) phase, at independent controlled flow rates. Then, by fixing the visualization at some oil ganglia they were able to reproduce and characterize the break up (fission) behavior observed by Hinkley showed in Fig. 2.11.

Vimieiro [13] used a technique similar to Weitz's group to investigate, using CLSM, the effect of injecting microcapsules in a porous medium after water injection. This work was already reviewed in the previous section.

The last part of the Theoretical Frameworks is dedicated to review the techniques and tools used in this work for image processing.

\section{5}

\section{Image processing}

This section aims to describe the tools used to prepare the CLSM images to their quantitative study.

Figure 2.36 presents a diagram that shows the canonical procedures used to extract quantitative information using image analysis. To grant good results, it is important that each step is designed to ensure obtaining its desired output, that serves as the input to the next step. 


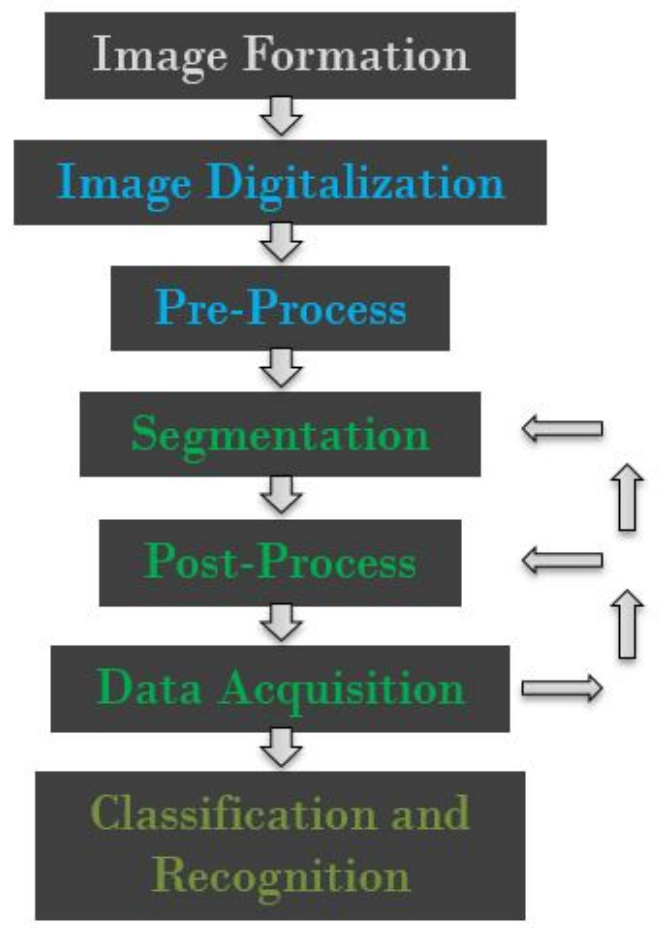

Figure 2.36: Classical diagram of image processing.

The image formation is the preparation needed to make possible a good image acquisition on the right area of visualization in the experiment. It comprises a good optical set up (illumination, lenses, cameras or photodetectors and the like) to acquire the image from the sample object. Then, the image digitalization occurs inside the camera, performing a transformation of a real image on a digitized, made of pixels filled with information. These cameras could, for example, generate 8-bits (gray scale) images or 24-bits (RGB colored) images. It is important to mention that, in this work, after this step, each original acquisition generates three distinct images, one for each phase present in the porous medium (oil, water and capsules). This is achieved by the use of different fluorescent pigments applied to each phase. Each pigment will be captured by a different photodetector.

Once the image is formed and digitized, it may be necessary a preprocessing step. That means to perform operations to correct the pixels intensity histogram, increasing the brightness and/or enhancing contrast, to apply filters to reduce the noise and to perform geometrical operations to improve the visualization on the focus of the work.

The segmentation is the critical step, where each particle will be distinguished from their neighbors and from the background, generating a binary image. There are a lot of methods to perform segmentation and each one will perform better in determined conditions. Illumination and pixel intensity 
defects usually interfere severely in this process. In this work, automatic segmentation methods were used, since the number of images to process is very high, making it impossible to perform segmentation for each plane image. The post-processing aims to correct mistakes that happened in the segmentation phase and to perform morphological operations, that further reduce noise in the binarized images. After that, the data acquisition allows the measurement of the desired parameters of the acquired image. It is important to notice, as shown in Fig. 2.36, that to get better results, segmentation, post-processing and data acquisition are usually applied in interactive loops. Also, in this work these three processes are designed to capture information exclusively for a given phase inside the porous medium.

Finally, the classification and recognition is the step that distinguishes each one of the phases that are in an image. In this work, this part of the image processing was not performed, since the classification was automatically performed in the previous steps.

All these image processing steps in this work were performed using two different programs, ImageJ and Dragonfly. Most steps were done using ImageJ, where, in order of application they were: filter application, segmentation (or binarization) and morphological operations. The macro developed to conduct these operations in ImageJ will be presented in the Experimental Procedure chapter.Dragonfly software was used only for data acquisition, because its features that make it easy to quantify volumes and to use advanced analysis tools.

These routines for image processing are described in details by Gonzalez and Woods [45], specially the morphological operations and the image segmentation methods. 


\section{Experimental Procedure}

This chapter focus on explaining the experiment design, experimental procedures and data processing protocols.

\section{1}

\section{General description of the experiments}

In this work experiments were performed to visualize two phase flow in porous media devices, composed of sintered glass beads, including the injection of water and a suspension of soft microcapsules to displace oil.

Figure 3.1 shows a simplified diagram of the experiment.

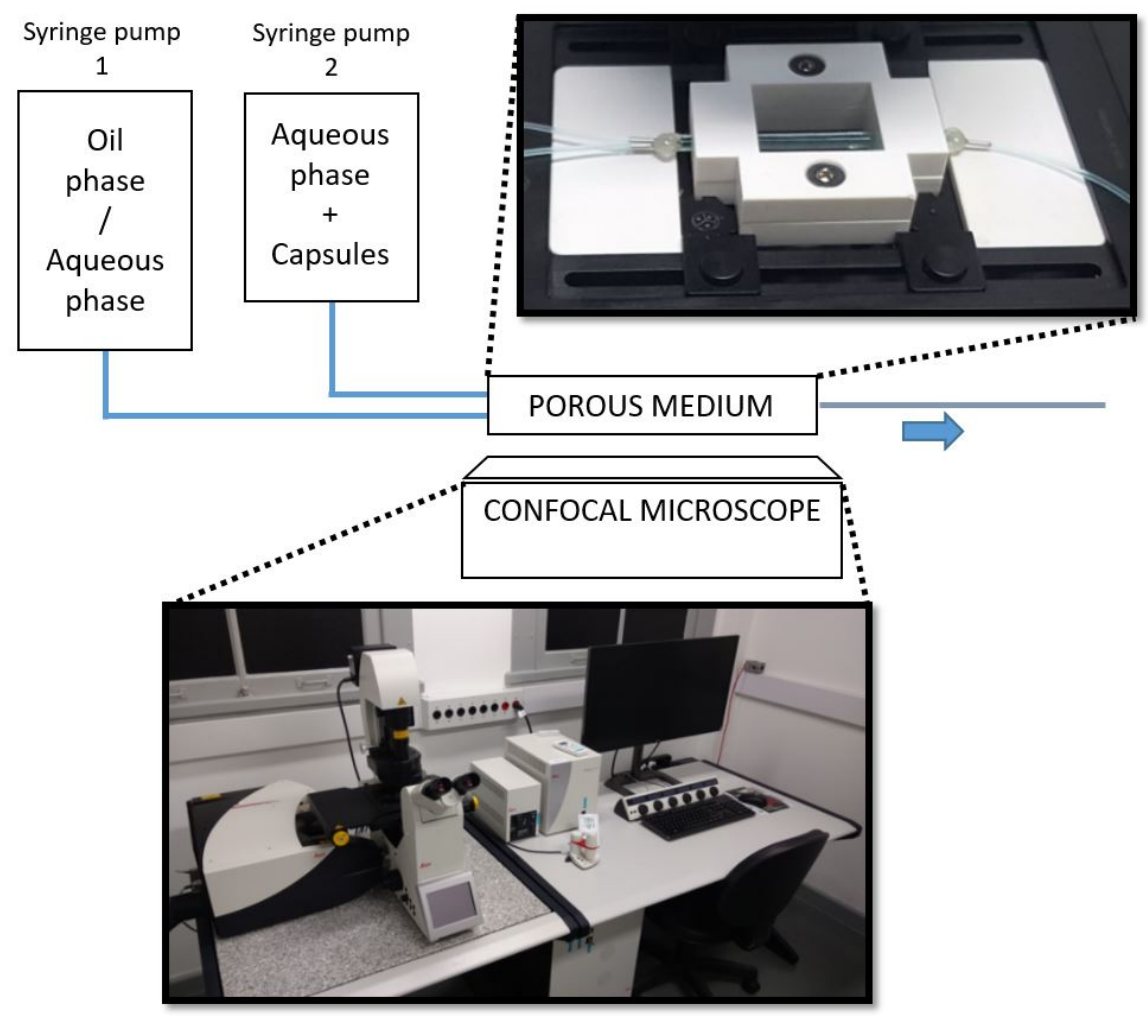

Figure 3.1: Simplified diagram of the experiment.

Two syringe pumps (Pump 11 Elite Programmable from Harvard Apparatus) injected the fluids into the porous media device. One of the pumps was used to inject both oil or aqueous phase; the other one injected a suspension of microcapsules. 
For the visualization of the experiment it was used a Leica TCS SP8 confocal laser scanning microscopy. The whole set up was located at the Laboratory of Microhydrodynamics and Flow in Porous Media (LMMP) at PUC-Rio.

These components, the fluids and the capsules used are further described in this chapter.

\subsection{1}

\section{Device fabrication}

The techniques used for the fabrication of the devices used in this work were inspired and adapted from the devices developed by Datta [20] and Vimieiro Junior [13]. The porous device model conceived in this work allowed the device to rotate around its longitudinal axes, making possible to visualize the porous medium from all sides. Also, the inlet of the devices was changed to be performed through a couple of needles. The needles allowed to reuse the device even if the injection tubes were damaged. Figure 3.2 shows a schematic illustration of the device. Other improvements were implemented in the porous devices and they will be described along this section.

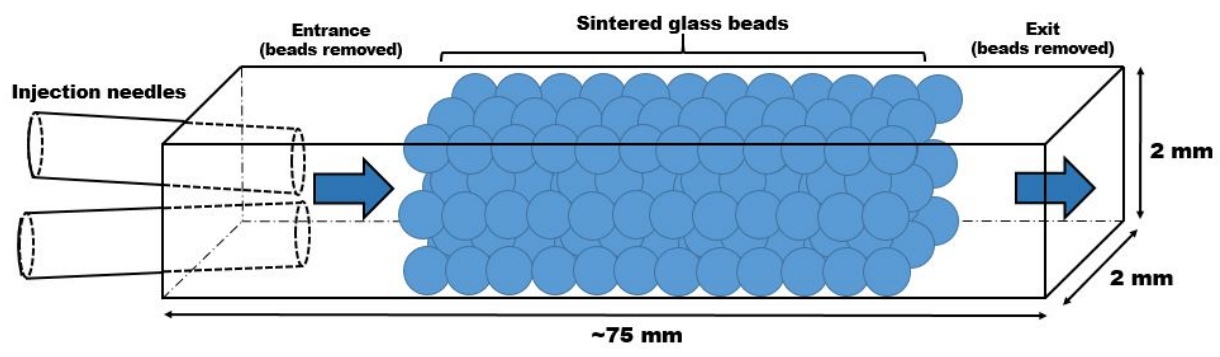

Figure 3.2: Schematic illustration of the porous device.

The porous media devices were produced by sintering borosilicate glass microspheres, fabricated by Mo-Sci, inside a quartz capillary with a square cross section with $2 \mathrm{~mm}$ side. This type of porous media device was chosen because it allowed visualization in depth, despite the fact that its properties differ in some aspects from rock cores.

The first step to produce the devices was, using a tungsten carbide scriber, to cut the square tubes and to identify them. The mean size of the square tube was $75 \mathrm{~mm}$ long.

Two types of devices were fabricated in this work. One was composed only by spheres of $425 \mu \mathrm{m}$ diameter; the other, called stratified (or twolayered), was composed by the combination of two layers, one with spheres of $425 \mu \mathrm{m}$ diameter, the other with spheres of $250 \mu \mathrm{m}$ diameter. Images 
obtained by microscope of the two types of porous media used on this work are presented in Fig. 3.3. Around fifteen devices homogeneous and stratified were built to perform the experiments in this work. Only six of those were used for the experiments reported in chapter 4 , four homogeneous and two stratified devices.
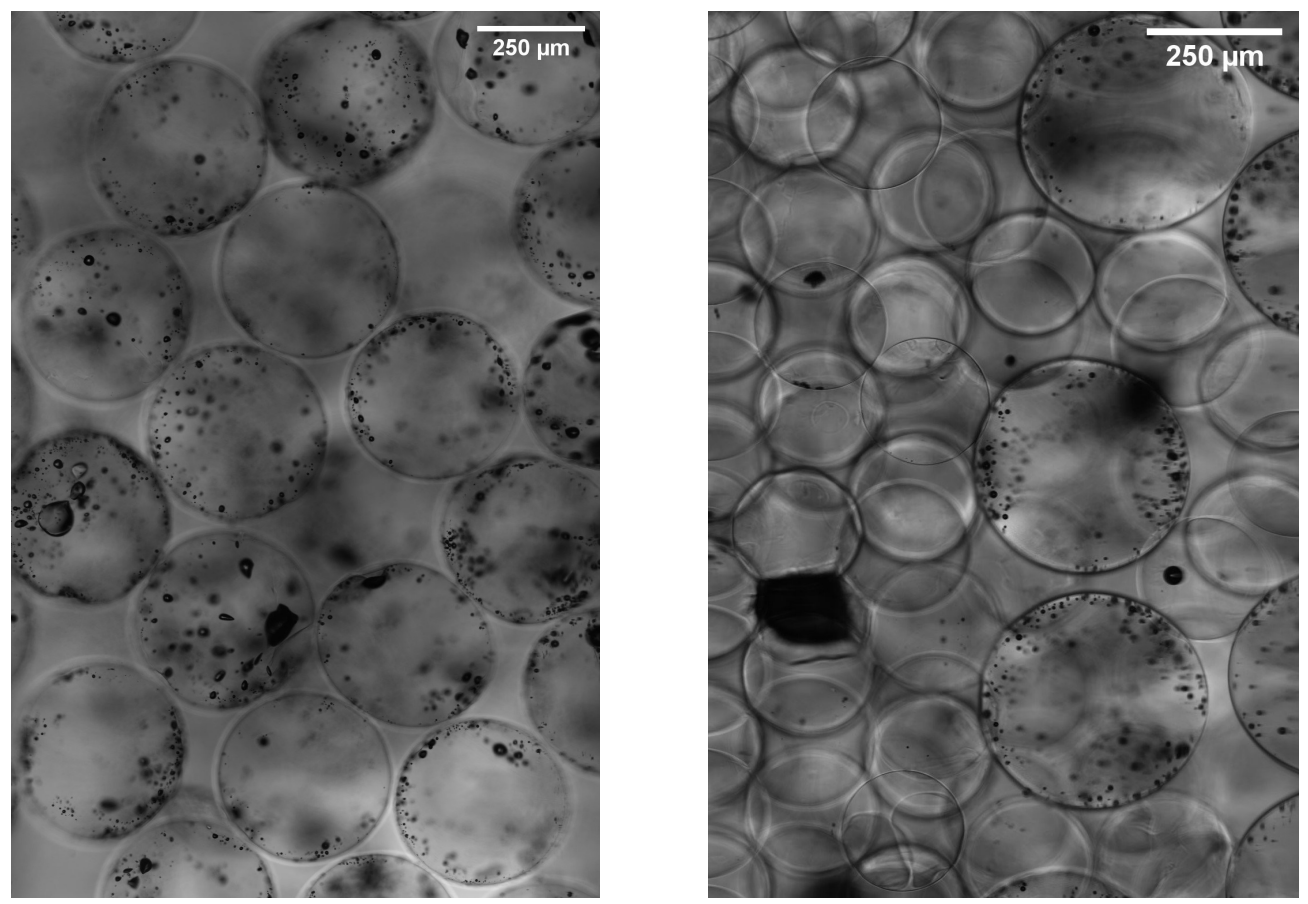

Figure 3.3: Microscope image of a porous media with glass beads of $425 \mu \mathrm{m}$ diameter (left) and a stratified (two-layered) porous media with 250 and $425 \mu \mathrm{m}$ diameter glass beads (right).

We chose the larger $(425 \mu \mathrm{m})$ beads considering the difficulties faced by Vimieiro [13] to make the capsules penetrate deep into a porous medium made of smaller spheres $(214 \mu \mathrm{m})$. The stratified porous media was developed to observe capsule injection and its effects in a non homogeneous medium.

In the fabrication of the single diameter glass beads porous devices, the square tubes were placed inside holes of a specially designed firebrick and then the tubes were filled with the glass beads. To achieve the temperature needed for sintering, a furnace from the Laboratório de Metalografia e Tratamentos Térmicos (LMTT) of PUC-Rio was used. The brick was placed in the furnace at $885^{\circ} \mathrm{C}$ for 8 minutes. A drawing of the brick is shown in Fig. 3.4. 


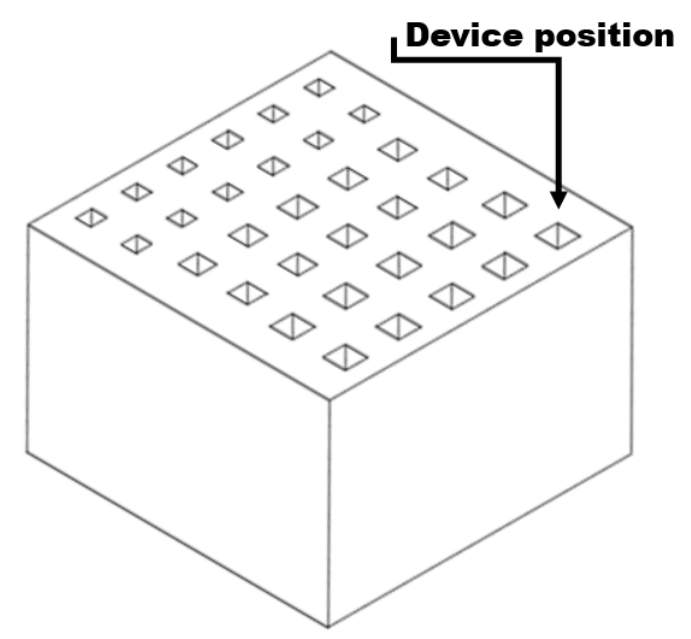

Figure 3.4: Model of the firebrick used to hold the porous device vertically on the furnace.

For the stratified porous devices, it was not possible to use the firebrick. If the square tube with two sized spheres was placed vertically in the brick, the porous media would be sintered with mixed microspheres sizes along the device, not in a two layered manner, as desired. Thus, the fabrication of a stratified porous device was done in two steps. First, the square tube was half filled with one size of glass beads. Then, the set was carefully placed on a horizontal smooth ceramic holder, which was resting on a brick, and everything was slowly moved into the furnace. If the capillary was laid to rest above an irregular surface, such as the brick, its walls would suffer small deformations, according to the resting surface holes. After staying 8 minutes inside the furnace, the set was removed and, when manageable again, after cooling, it was filled with the other size of microspheres and placed on the furnace one more time for 8 minutes. The set up with capillaries, ceramic holder and brick is shown, when cooling, in Fig. 3.5. 


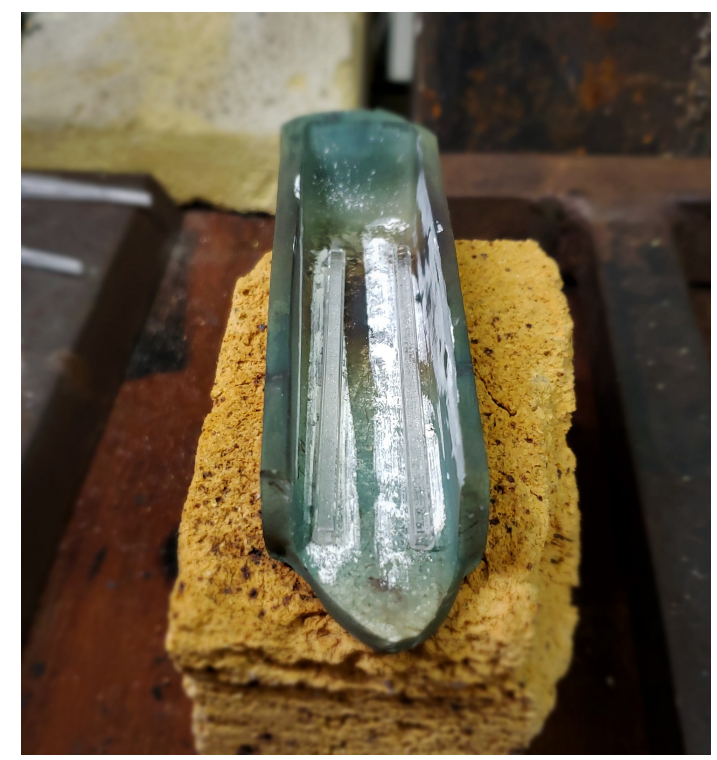

Figure 3.5: Device cooling before the second sintering process for the stratified porous device.

Another difference from the Vimieiro's and Datta's devices was that, after the sintering process, glass beads were removed from regions at the entrance of the device, as can be seen in Fig. 3.2. This was done both to create an space to the injection needles and to have a distribution chamber region before the porous medium. The same was done at the exit of the device, so to have a space to the exit needle (not shown in Fig. 3.2).

The porosity of the devices were, then, measured. The technique was first to weight the porous device filled with air and, then, completely saturated with water. Thus, knowing the water density, was possible to calculate the porosity. It is important to notice that, to guarantee that the porous medium was free from air bubbles, it was saturated by capillary imbibition. Table 3.1 shows the porosity range for the devices developed in this work and some values for similar porous media models. Both the single sized spheres devices and the stratified ones had similar values of porosity.

Table 3.1: Porosity range of the devices developed in this work and the ones presented in the literature.

\begin{tabular}{cc}
\hline & Porosity (\%) \\
\hline This work & $39-42$ \\
Sahimi [22] & $36-43$ \\
Carman [23] & $35-40$ \\
Datta [20] & $\approx 41$ \\
\hline
\end{tabular}

Following the porosity calculation, needles were fixed at the entrance and 
exit of the device, allowing to connect directly the tubes from the injection syringes. They were carefully cut from a commercial syringe needle using a pair of pliers. After selecting the ones that were not bent or had the cross section obstructed, the needles were placed in the capillary square tube and epoxy glue was applied. Figure 3.6 shows the needle used for the connection. Using this procedure it was possible to produce reusable and precise devices.
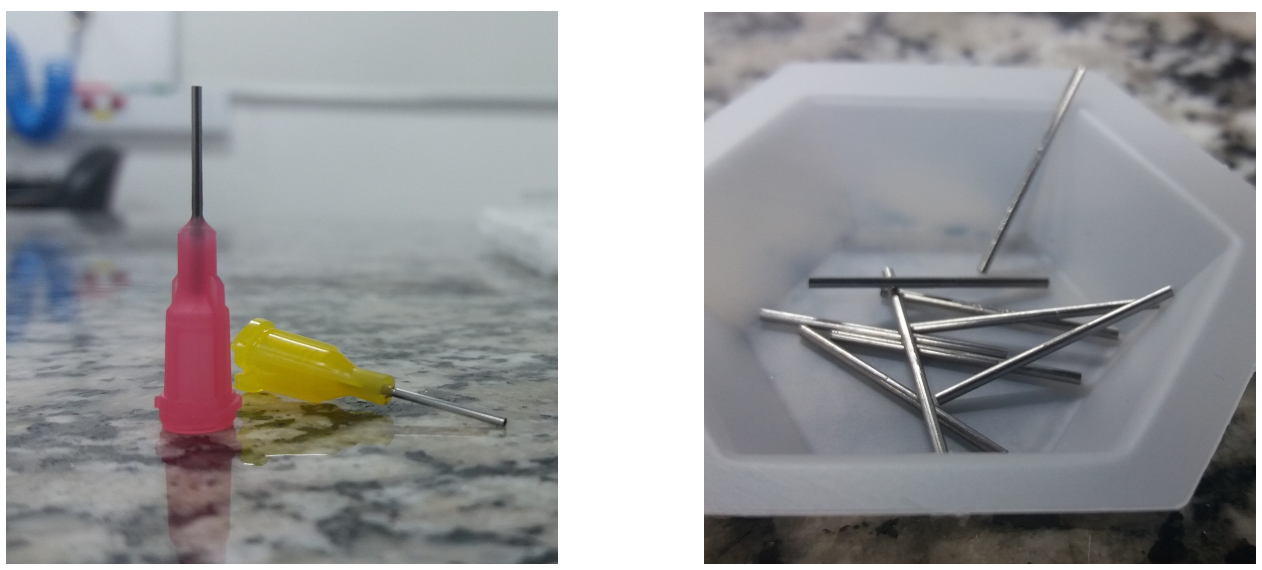

Figure 3.6: Needles used in the laboratory for syringe-tube connections and their parts removed to manufacture the porous media device connection.

In addition to the porosity, another property of the porous media important for flow analysis is the permeability. Thus, an experiment of water injection at different flow rates on each porous device was performed. A Fluigent system, composed by a Flow EZ 2000mbar and a Flow Unit M, was used in order to control and measure the pressure and the flow rate, respectively. As the objective of this test was only to measure the permeability, no visualization was acquired at the confocal microscope.

In the measurement of the present experiments, the pressure drop for calculating permeabilities also includes the part of the injection capillary, the inlet and outlet needles and the region of the device before the porous medium. Thus, first was performed a measurement of pressure drops without the porous medium. Then, for calculating the permeabilities, the measured pressure drops with the porous medium were subtracted from the pressure drop of the system without the device.

The range of permeability measured in this work is presented in Table 3.2, along with Datta's [20] and Vimieiro's [13] devices permeability. Also, an estimation using Kozeny-Carman relation, Eq. 3-1 was calculated to assure agreement:

$k=\frac{1}{C_{k}} \frac{\phi^{3} d_{s}^{2}}{(1-\phi)^{2}}$, 
where $d_{s}$ is the diameter of the sintered glass spheres and $C_{k}$ is a constant that, according to the literature, may vary a lot (from 45 to 180, [20] [46]). In this work a value of 180 for $C_{k}$ was used.

Table 3.2: Permeability from the devices developed in this work compared from the devices used by Carman [23] and Data [20]

\begin{tabular}{|c|c|c|}
\hline & \multicolumn{2}{|c|}{$\begin{array}{c}\text { Permeability } \\
\text { (Darcy) }\end{array}$} \\
\hline & Measured & Kozeny \\
\hline This thesis $(425 \mu \mathrm{m})$ & $190-230$ & $\approx 180$ \\
\hline This thesis (250 and $425 \mu \mathrm{m}$ ) & $180-190$ & $\approx 120$ \\
\hline Datta $[20](38 \mu \mathrm{m})$ & 1.69 & - \\
\hline $\operatorname{Datta}[20](150 \mu \mathrm{m})$ & 76 & - \\
\hline Vimieiro [13] ( $214 \mu \mathrm{m})$ & 12.6 & - \\
\hline
\end{tabular}

As expected, devices with larger glass beads presented higher values of permeability. In other words, the fluid flows easier inside devices with larger glass spheres.

Finally, after the device fabrication and characterization were completed, a holder was designed to support it in the microscope. Therefore, the porous device and microscope stage were measured and different models of holders were designed. Figure 3.7 shows the preliminary chosen design and its dimensions. The holder was meant to make possible multiple acquisitions without losing the scanning position and it allowed to axially rotate the device in the microscope. Thus, images from both sides of the porous device could be obtained.

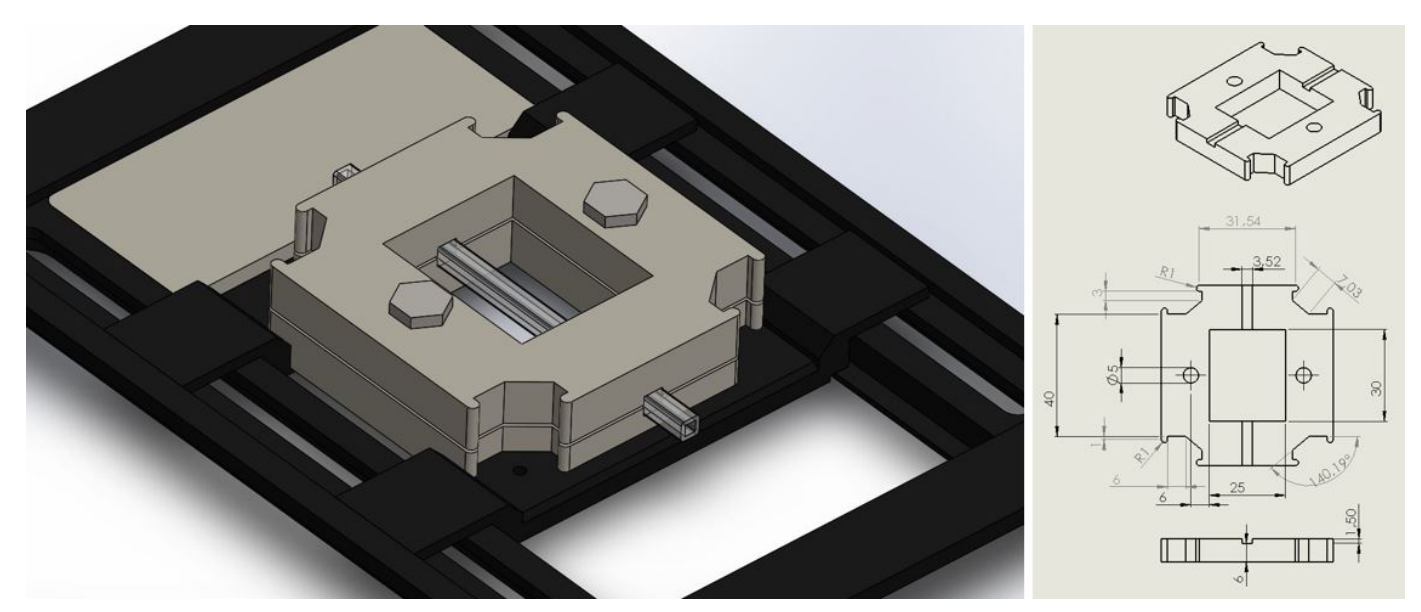

Figure 3.7: 3D view from the device holder and its measures.

Briefly, the differences between the devices developed in this work and the one used by Vimieiro [13] were: the use of a designed firebrick for the sintering 
process; the sintering of two layered porous media; the use of needles to make the tube connections; and the substitution of the glass slide by a designed holder to position the device in the microscope. Some of these changes are clear in Fig. 3.8.
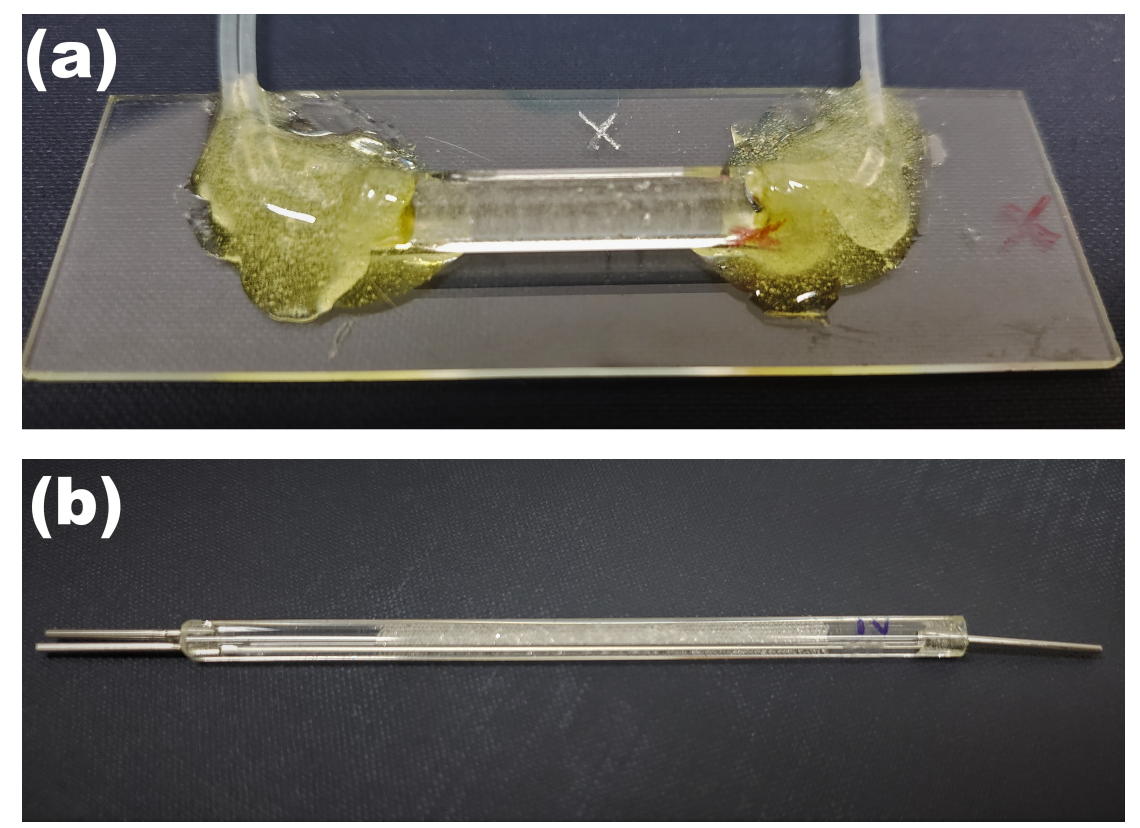

Figure 3.8: Difference between previous porous devices used by Vimieiro [13] (a) from the one developed on this work (b).

Observing the differences between the porous devices, shown in Fig. 3.8, it is also possible to notice that in the device developed in this work uses a much smaller amount of glue to guarantee the sealing.

\subsection{2}

\section{Device holder}

As mentioned before, a device holder was developed, allowing to flip the porous device in the microscope and visualize it from different sides. The holder was designed to have a precise fabrication and to fit tightly on the microscope stage, eliminating undesirable movements.

An image of the device placed on the microscope stage is presented in Fig. 3.9. 


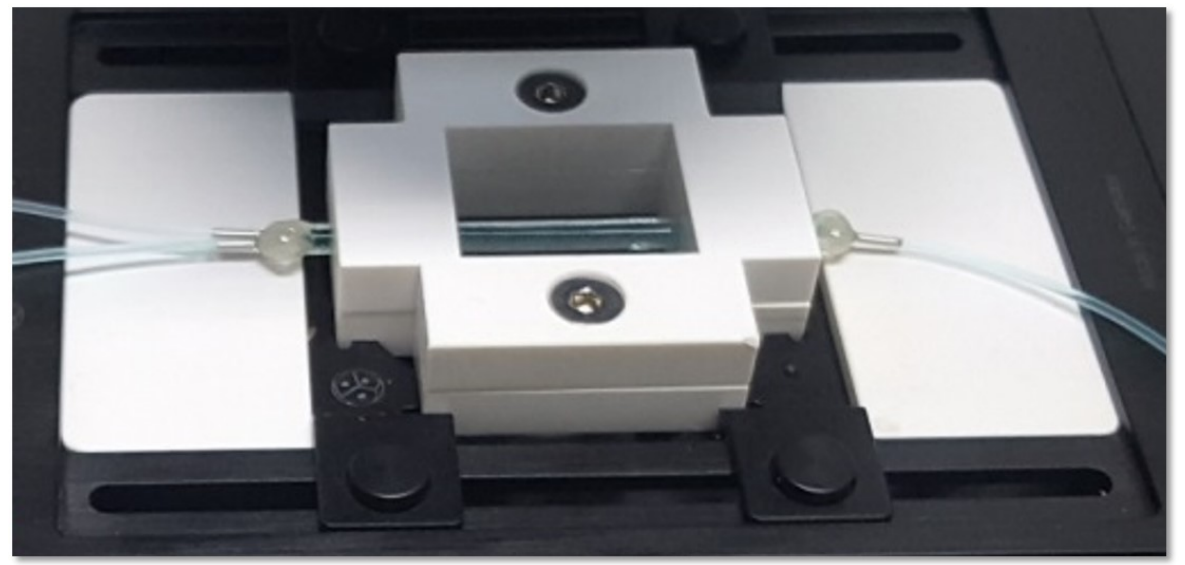

Figure 3.9: Device holder placed on the microscope stage.

As the experiments were carried on, it was found that an important region for analysis was the entrance of the device. Unfortunately, as presented in Fig. 3.9, the device holder blocks the visualization of this region. Then, to remediate this problem, the device was moved so that the holder covered only its exit. In the future, another holder will be built so that this problem will not occur. Figure 3.10 show how the device was positioned in the holder in the experiments.

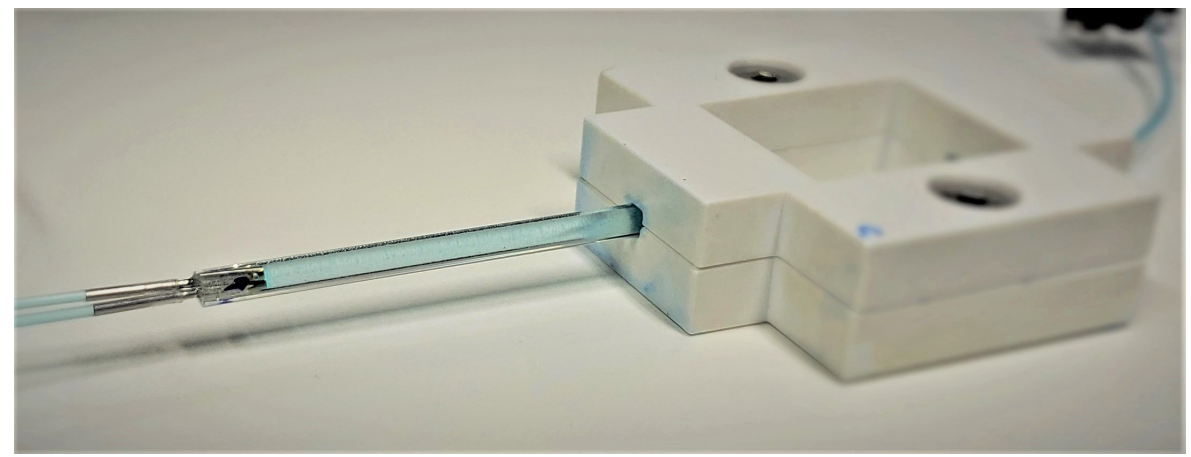

Figure 3.10: Adaptation on positioning the porous device in the holder.

By using this device holder positioning adaptation, it was not possible to use the stage spacers (shown in Fig. 3.9), so every test was performed placing the holder at the corner of the microscope stage.

\subsection{3}

\section{Fluid choice}

On preliminary experiments, the same oil and aqueous phases composition used by Vimieiro [13] were used. The aqueous phase was a solution of dimethyl sulfoxide (DMSO), sucrose and purified water, the oil phase was a mixture of an immersion oil and a lubrication oil. With these, Vimieiro achieved 
the same refractive index and the desired result on the miscibility test. The viscosity of each phase was $\mu_{w}=34[\mathrm{mPa} . \mathrm{s}]$ for the aqueous phase and $\mu_{o}=106$ [mPa.s] for the oil phase, both at $24^{\circ} \mathrm{C}$. Between these phases the interfacial tension was $\gamma=22.2[\mathrm{mN} / \mathrm{m}]$.

In order to force the microcapsules inside the device, it was found that the injection flow rate had a strong effect on how deep the capsules would penetrate into the porous medium. However, to maintain a high residual oil saturation after an increase in the flow rate, a variation of the fluids used by Vimieiro was explored. To reach that goal, a higher viscosity ratio was desired. On the aqueous phase, it was found that the composition had already a lot of DMSO, which was used to increase the refractive index and lower the viscosity. Solutions with higher concentration of DMSO affected the capsule durability, so the aqueous phase composition was not changed. Therefore, other oils available in the laboratory were checked in a search for one with high refractive index and viscosity. It was found that the Castor Oil, fabricated by Mundo dos Óleos, had almost the same refractive index of the glass beads and a much higher viscosity than the previous oil phase solution, $\mu_{o}=755[\mathrm{mPa.s}]$, at $24^{\circ} \mathrm{C}$. With the Castor Oil, the viscosity ratio increased from $\approx 3$ to almost 23. The interfacial tension between the aqueous phase and the Castor Oil, at $24^{\circ} \mathrm{C}$, was $\gamma=21.8[\mathrm{mN} / \mathrm{m}]$.

Then, a miscibility test was done and the Castor Oil and the aqueous phase are not miscible. The result of the miscibility test is shown in Fig. 3.11, it is possible to observe the phase separation after the solution was mixed. 


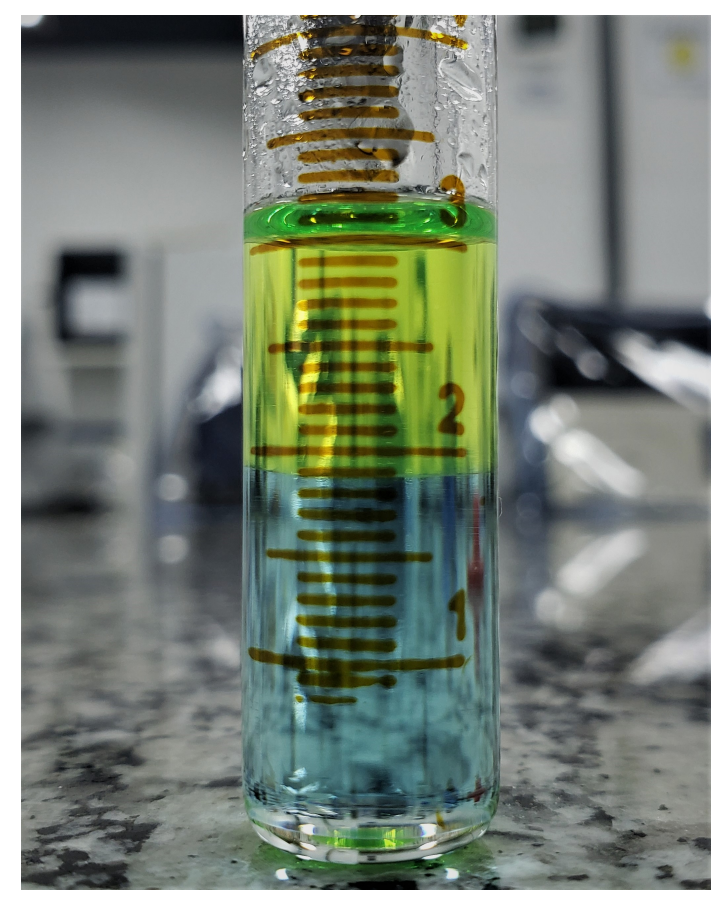

Figure 3.11: Oil and aqueous phase distinguished on the miscibility test. Aqueous phase is dyed light blue and the oil phase is dyed in yellow.

So, the aqueous phase composition used by Vimieiro [13] was kept the same: Dimethyl Sulfoxide (54\%), Sucrose (32\%) and Milli Q Water (14\%). For the oil phase, Castor Oil was used.

\subsubsection{1 \\ Refractive index}

As explained earlier in the Theoretical Framework chapter, an important parameter to the fluid choice is the refractive index. To ensure a good depth of visualization using the CLSM technique is mandatory that all the objects of analysis have the same refractive index. In other words, the refractive index of the glass beads and both fluids had to match.

In Vimieiro's work, the methodology used to measure the glass beads refractive index was to test the glass beads immersion in fluids with known refractive index, as shown in Fig. 3.12. A transparent vial was placed on a writen paper and then filled with the glass spheres. After that, fluids with different refractive index were dipped in the vial and the one which granted the clearer visualization of the paper was the one that matched best the refractive index of the beads. 

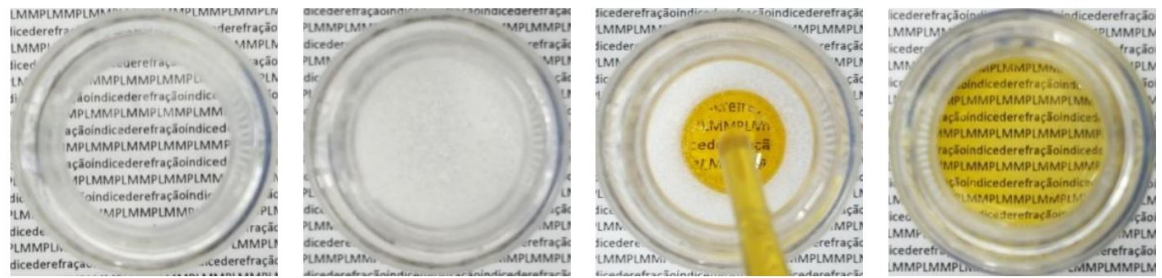

Figure 3.12: Method used by Vimieiro [13] to match the solids and fluids refractive indices.

Another possible method to measure refractive indices was the one described by Yue, Wang and Huang [21]. This procedure was useful because it allowed to obtain the refractive index of the microspheres in a faster way than the other method mentioned. First, the refractive index from an immersed fluid was measured with an Abbe refractometer. Then, a small amount of glass spheres were placed inside a vial filled with this fluid. This setup was placed in an optical microscope and after some images were acquired, using the formulas described in Yue et al., it was possible to calculate the spheres' refractive index. The idea is to correlate the refractive indices with the radii observed in a microscope, as shown in Fig. 3.13. This method is very flexible, one only needs to know one of the refraction indices (fluid or spheres) to calculate the other. Figure 3.14 shows an image obtained in this work for the measurement of the refractive index following the Yue et al. method.
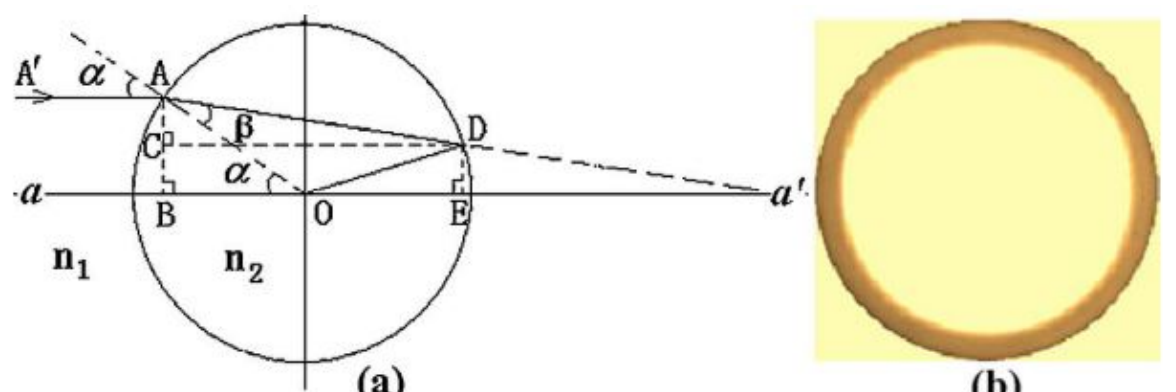

(b)
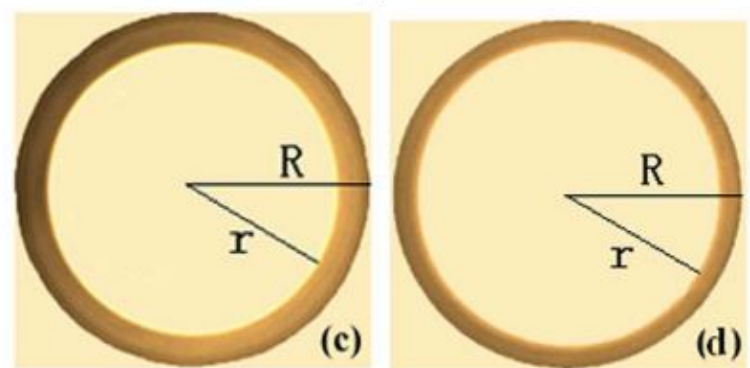

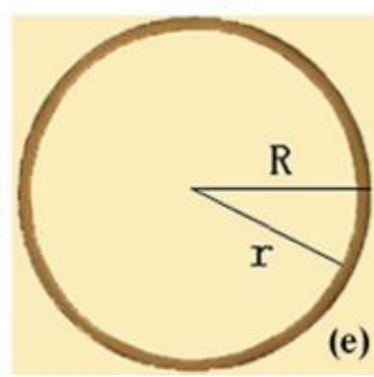

Figure 3.13: Diagram of how the method of Yue et al. [21] can determine the refractive index of the microsphere (a). Microsphere image obtained by phase contrast microscope (b). Images of a polystyrene microsphere immerserd in water (c), ethanol (d), and a 1:1 mixture of glycerol and water (e). 


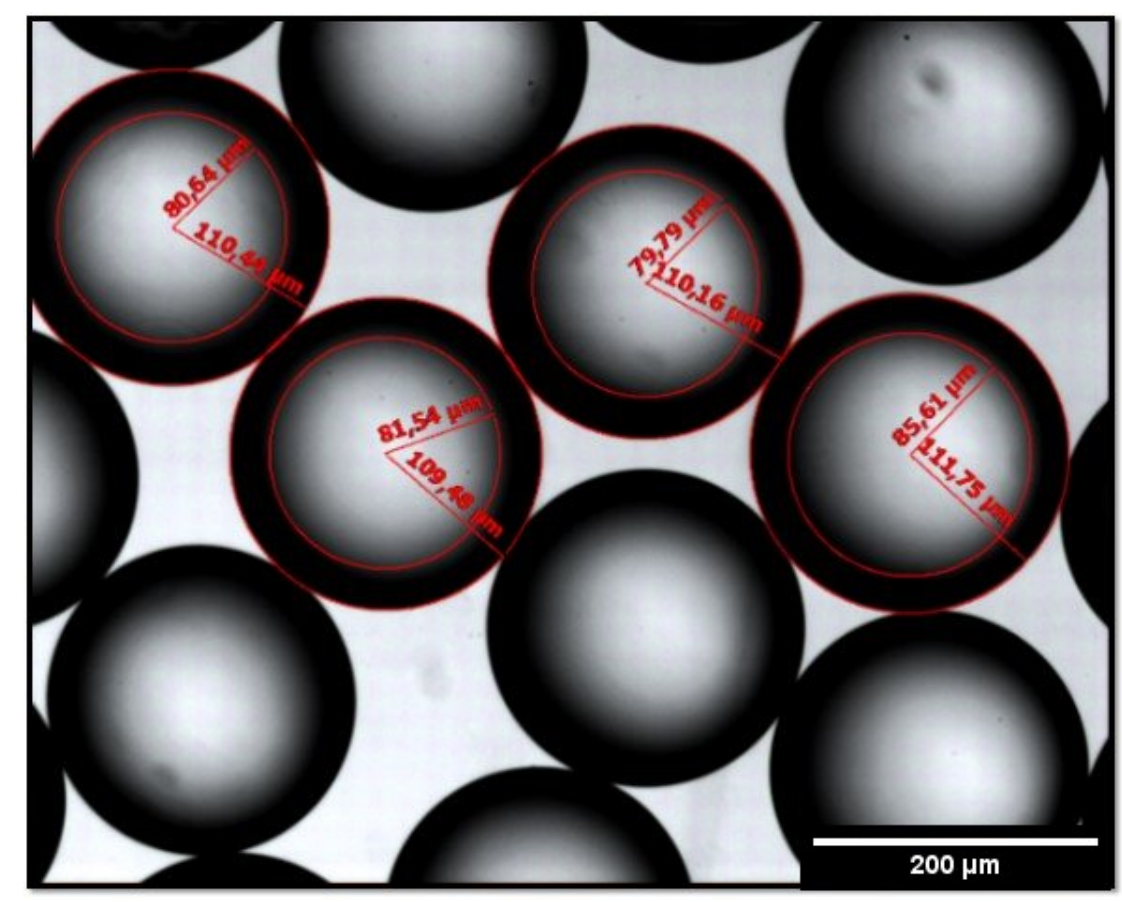

Figure 3.14: Measurement of the refractive index of glass beads by the method proposed by Yue et al. [21].

In this work, first it was applied the methodology proposed by Yue et al., since it achieves a faster/coarse value for the refractive index. After this preliminary screening, the method used by Vimieiro [13] was used to fine tune the results.

At the end, it was found that the glass beads used on this work had a refractive index similar to those used by Vimieiro [13], $\eta=1.478$. This was expected, since both were produced by the same company and their main difference, besides their sizes, was that the $425 \mu \mathrm{m}$ beads had some small imperfections, seen as small black dots on Fig. 3.3. Both the aqueous and oil phases had the same refractive index.

\subsubsection{2}

\section{Fluorescent pigments}

The use of fluorescence is a must to visualize the different phases. After excited by lasers of given wavelengths, each phase (mixed with a suitable fluorescent pigment) emits light at distinguishable wavelength.

In the works by Datta [20] and Vimieiro [13], they used a fluorescent pigment diluted only on the aqueous phase. So, the quantification of the other phases was done by subtraction from the device fully saturated with the aqueous phase. In this work, as mentioned in the motivation, it was proposed to visualize simultaneously the two phases and the microcapsules. 
Therefore, three different fluoresce pigments were needed: one soluble in water, for the aqueous phase and two soluble in oil, one for the oil phase and the other for the innermost (oily) phase of the microcapsules. By that, it is possible to separate the emissions and direct them to different detectors.

The choice of the fluorescent pigments to be used in each phase and inside the capsules was done very carefully in order to minimize wavelength overlapping problem. Briefly, to assure that visual distinction of the different phases and capsules was possible (each with well defined and distinct emission wavelength peak), one, or preferably both, of these two conditions must be achieved in the selection of the fluorescent dyes:

- the emission spectrum of each pigment has a good separation from the others and low superposition when excited by the same laser wavelength; or

- for a given laser excitation wavelength, only one phase responds to it.

In fact, the second condition is more restrictive than the first one and, in the scope of this work, all selected pigments obeyed it.

For the aqueous phase detection some alternatives were already known, as Rhodamine-B and Methylene Blue.

The hardest part to obtain simultaneous visualization was to distinguish the oil trapped in the porous media from the oil that was inside the gellan gum capsules. Achieving that, it was possible to visualize the distribution of the microcapsules in the device after a two phase flow experiment.

To accomplish that, a survey was performed to find compatible fluorescent pigments soluble in oil. Usually, this type of product is extremely expensive and comes in minimal quantities. This happens because the market is focused on typical applications of fluorescent microscopy, such as to visualize small cells and organelles. However, a company, Spectroline, develops fluorescent pigments for industrial use, to detect leaks on circulating fluid systems. It is used, for example, to find leaks in fuel, lubricant, petroleum, water and water/glycol-based systems.

An oil leak detection kit was delivered by the company to test the pigments for the oil phase and the capsules, and later the aqueous phase pigment was chosen according to the oily pigments. This oil leak detection kit was composed by 7 small vials with samples of diluted fluorescent pigments, those vials are shown in Fig. 3.15. 


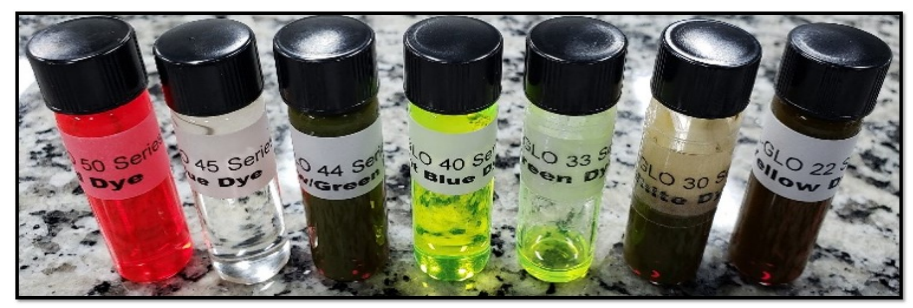

Figure 3.15: Fluorescence leak detection kit, from Spectroline, used to perform excitation/emission tests the distinction on the oil phase and microcapsules.

Each one of these solutions were tested with the lasers attached in the confocal microscope used in this work. Since the kit was not developed for simultaneous visualization, the pigments usually presented large emission spectra overlaps. This made harder the choice of the pigments, since these overlaps had to be avoided.

The results of this survey for oily pigments is shown in Fig. 3.16. There are the emission spectra of different fluorescent pigments for different excitation laser wavelengths, guiding to a solution that allowed the simultaneous visualization of the three phases. Again, the idea was to get emission peaks in different wavelengths for each phase. This can be achieved for the oil phase with OilGlo 33 (excited by $488 \mathrm{~nm}$ laser); the capsules with OilGlo 30 (excited by $552 \mathrm{~nm}$ laser) and the aqueous phase with methylene blue (excited by 638 $\mathrm{nm}$ laser). Important to notice that it was not possible to perform simultaneous acquisition for the three phases as the confocal microscope was equipped with only two photodetectors. This requires a sequential acquisition: first the aqueous phase and the oil phase were mapped in the images and later the microcapsules. 


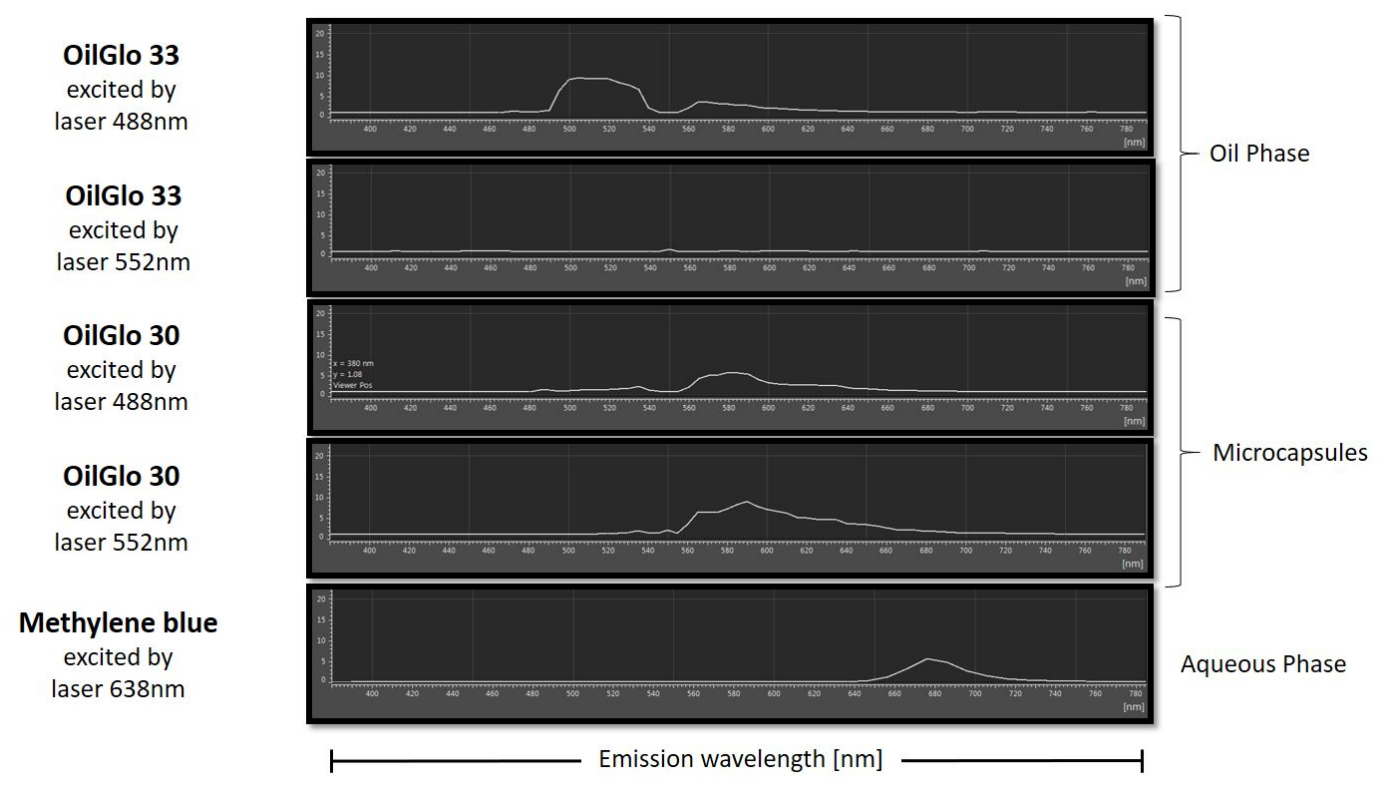

Figure 3.16: Emission spectra from different fluorescent pigments after 488nm, $552 \mathrm{~nm}$ and $638 \mathrm{~nm}$ wavelengths laser excitation.

Figure 3.17 shows in more details the range used for each detection and 3D perspective views for the oil phase and microcapsules distinguished.

OilGlo 33

Laser $488 \mathrm{~nm}$
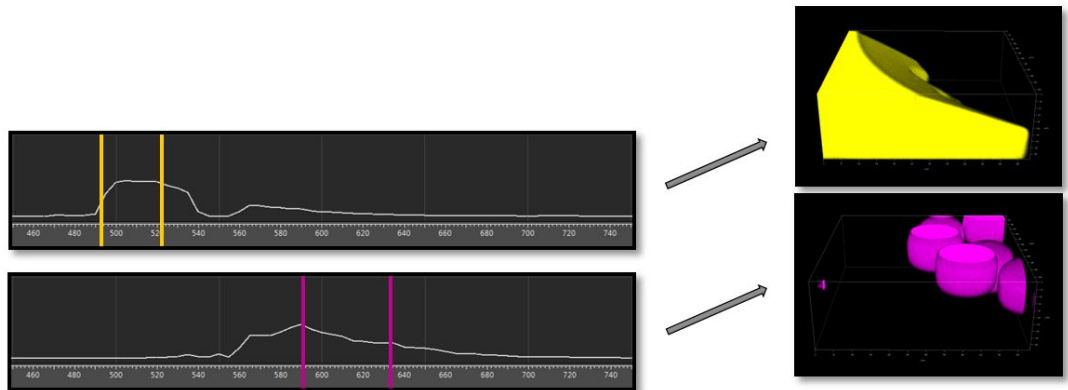

OilGlo 30

Laser $552 \mathrm{~nm}$

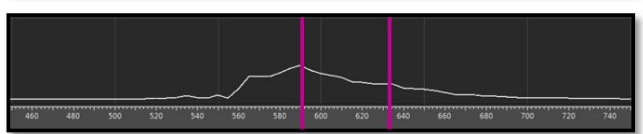

Methylene Blue

Laser 638nm
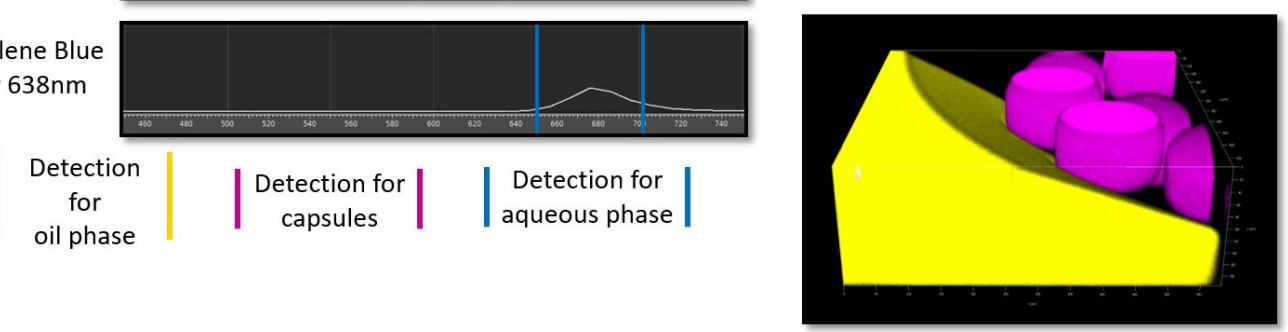

Figure 3.17: Spectra and range of detection for each fluorescent pigment chosen. Separated and combined visualization of the oil phase (yellow) and the microcapsules (purple).

Further in this chapter the detectors selection and the acquisition sequence will be explained in more details. 


\subsection{4 \\ Microcapsules}

The procedure for fabricating the microcapsules was developed by colleagues from LMMP. The set up is described in more details in Michelon et al. work [14] and was briefly summarized on the Theoretical Framework chapter. Michelon et al. developed a research of microcapsules production conditions for different dimensions.

Based on the technique for microcapsules formation, the first step was to fabricate proper microfluidic devices (like those shown in Fig. 2.24) with dimensions designed to reach the desirable shell thickness and diameter for use in this work.

For each device, a pair of glass capillary tubes with pointed ends was needed. They were produced using a micropipette puller (model P1000, Sutter Instrument Co.). The measurement of the tips' diameter was ensured by carefully grinding it. After that, the hydrophilic and hydrophobic treatment was done separably on each capillary. Then, a microscope was used to allign the capillaries inside the square capillary and guarantee the positioning after applying the glue (fabricated by Devcon Corp.). The last step on the microcapsule producing device fabrication was to cut and glue the needle connection where each phase passes.

Before producing the capsules, the device was not used for one day to ensure proper cure of the glue. Then, the setup with three pumps (model Pump 11, Harvard Apparatus) and the high speed camera (model Fastcam SA-3, Photron) were placed. The flow rate of each phase was established and, after some time when the stable regime started, the capsules were collected in vials, as described on the Theoretical Framework chapter. The setup with the syringe pumps, miscroscope, high-speed camera and the computer is shown in Fig. 3.18. 


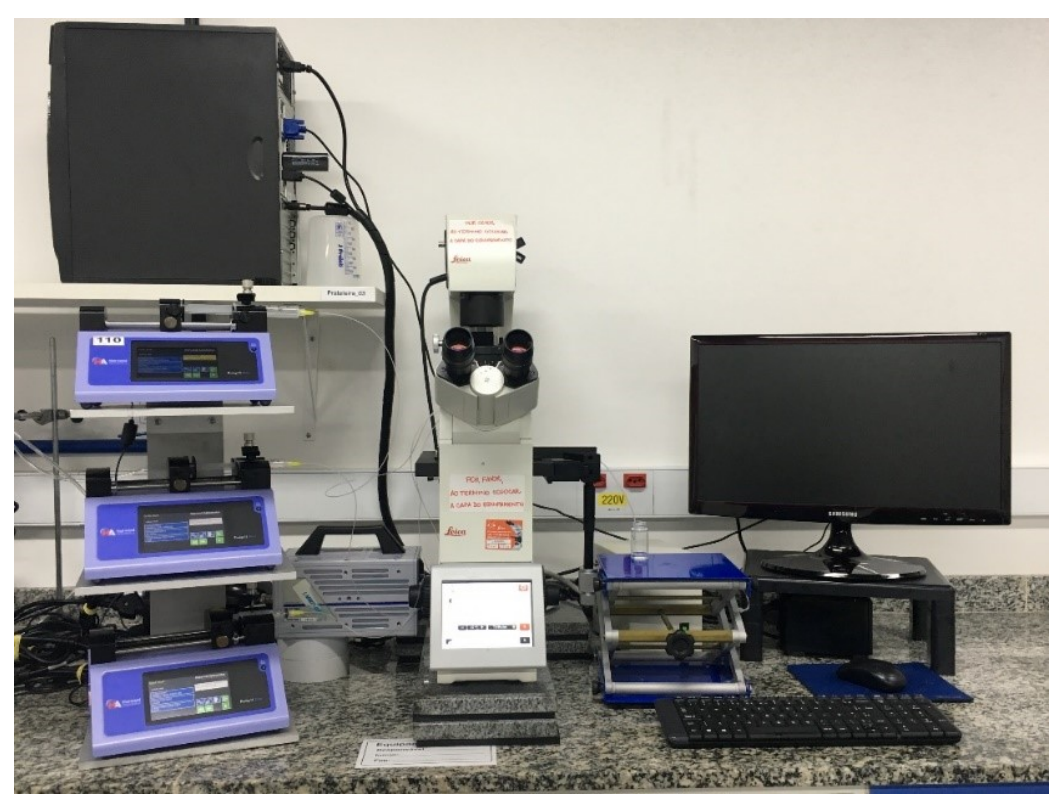

Figure 3.18: Microcapsules fabrication setup with three syringe pumps, an inverted microscope, a high-speed camera and a computer.

Using the device Design \#2 of Michelon et al. [14], a variety of capsules batches were produced. The list of microcapsules fabricated with this device and with the fluorescent pigment mixed in the inner phase is shown in Table 3.3. The Device \#2 parameters are, as shown in Fig. 2.24, the inner diameter of the inlet capillary and outlet capillary, $50 \mu \mathrm{m}$ and $250 \mu \mathrm{m}$, respectively, and a distance of $75 \mu \mathrm{m}$ between them.

Table 3.3: List of microcapsules produced using the Device \#2 and the fluorescent pigment mixed in the inner phase of the capsules.

\begin{tabular}{ccc}
\hline Code & Diameter $[\mu \mathbf{m}]$ & Thickness $[\mu \mathbf{m}]$ \\
\hline UU1 & 104 & 5 \\
OO3 & 108 & 4 \\
ZZ1 & 117 & 4 \\
OO2 & 122 & 5 \\
VV1 & 128 & 5 \\
XX1 & 129 & 5 \\
QQ2 & 130 & 8 \\
OO1 & 135 & 5 \\
PP2 & 140 & 12 \\
QQ4 & 142 & 11 \\
QQ1 & 145 & 10 \\
PP1 & 154 & 15 \\
LL5 & 202 & 7 \\
\hline
\end{tabular}


The capsules were stored in refrigerated vials with water, for conservation.

The choice of the microcapsules diameter was based on theoretical estimation of pore channel size. Dias et al. [47] proposed a formula, Eq. 32 , that correlates the average pore size with the diameter of the glass beads and the porosity, $\phi$, of the medium:

$d_{p}=2 d_{s} \frac{\phi}{3(1-\phi)}$

where $d_{p}$ is the average porous channel and $d_{s}$ is the diameter of the glass spheres.

Equation $3-2$ lead to an average pore size of $\approx 200 \mu \mathrm{m}$ for the $425 \mu \mathrm{m}$ glass beads pack and $\approx 120 \mu \mathrm{m}$ for the $250 \mu \mathrm{m}$ spheres.

Another method to estimate the porous channel size is calculating for each type of arrangement of packed spheres. The arrangements could be simple cubic, body centered cubic or face centered cubic. Those patterns are not precise models for the whole porous device, as they estimate porous volumes much different from the ones observed on the fabricated media. In any case, these estimations lead to minimal porous throats between 65 and $175 \mu \mathrm{m}$.

With both estimations at hand, in order to increase the chance of forcing the microcapsules further inside the porous medium, it was chosen to use the smaller and thinner microcapsule. So, in the experiments described in this work the microcapsules used were from batch UU1 (Table 3.3) with $104 \mu \mathrm{m}$ of diameter and $5 \mu \mathrm{m}$ of shell thickness. This batch was farmed using the flow rates $Q_{i}=100 \mu l / h, Q_{m}=50 \mu l / h$ and $Q_{o}=3500 \mu l / h$.

Just to verify, some tests were performed using other batches (such as LL5, with $202 \mu \mathrm{m}$ of diameter and $7 \mu \mathrm{m}$ of shell thickness, and QQ1, with 145 $\mu \mathrm{m}$ of diameter and $10 \mu \mathrm{m}$ of shell thickness), but they did not lead to good penetration of microcapsules. So, only experiments with UU1 microcapsules are reported here.

\section{2}

\section{Procedure}

In this section the experimental procedure is described. The experiment could be divided in three steps according to the fluids' injection into the porous device: saturation of the device with the oil; aqueous phase injection; and injections of the microcapsules suspension together with the aqueous phase.

The setup was already shown in Fig. 3.1. It was set that the entrance tube \#1 was used for the aqueous and oil phase injections and the entrance 
tube \#2 for the microcapsules suspension injection.

\subsection{1}

\section{Device saturation with oil}

Once the device was sintered and with the connections glued, microfluidic tubes were measured and connected to the device. One of the tubes was connected to the laboratory's $\mathrm{CO}_{2}$ line and, after 15 minutes of injection at $69 \mathrm{kPa}$ manometric, an aqueous phase syringe was connected to the exit tube of the device and its content was forced into the device. By this, the saturation of the device with aqueous phase was guaranteed without presence of air bubbles. A CLSM image of the device fully saturated with the aqueous phase was acquired at this point. The configuration of the Leica Acquisition Software (LAS X) for this acquisition will be described further in this chapter.

After this step, the microcapsules were positioned inside the tube connected to tube entrance \#2. This was done collecting capsules from the vial where they were stored using a plastic pipette and dropping them on a concavity slide, so to avoid contamination of those remaining in the vial. Then, the capsules on the slide were sucked up by the tube connected to the syringe filled with the aqueous phase. Once they were inside, the tube was connected to the device thru tube entrance \#2. Then, the flow from this tube was blocked using a binder clip to guarantee that the microcapsules would not enter the device before the right moment.

The device was then saturated with the oil phase through tube $\# 1$, initially at a steady flow rate of $1000 \mu \mathrm{l} / \mathrm{h}$. Then the flow rate was increased manually to guarantee an almost full saturation (up to the water irreducible saturation).

A diagram explaining this step is shown in Fig. 3.19. 


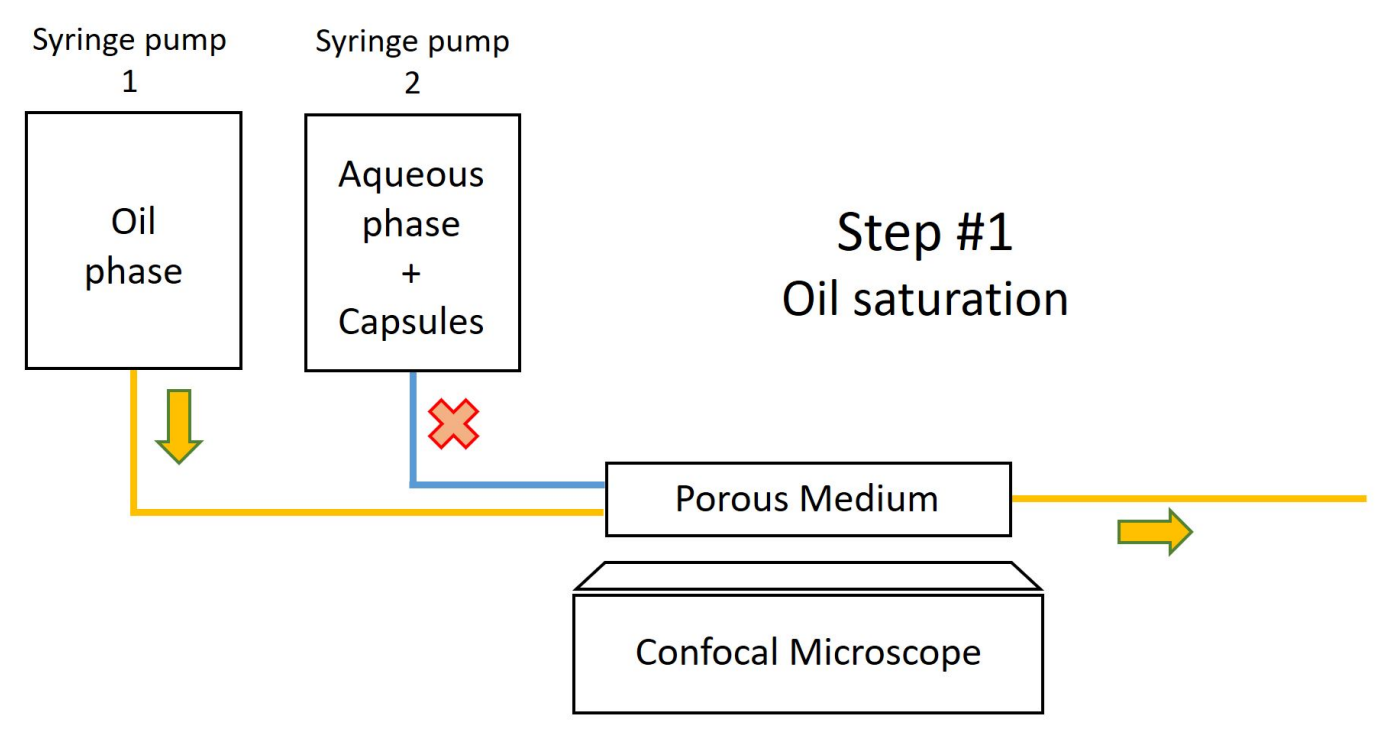

Figure 3.19: Diagram explaining the procedure for oil saturation of the porous device. The line filled with oil phase is shown in yellow and the line with aqueous phase is shown in blue.

After the device saturation an image was obtained to analyze the pore structure on the region under study.

\subsection{2 \\ Injections procedure}

Once the device was fully saturated with oil, it was ready to start the oil displacement tests. The first step was aqueous phase injection at $100 \mu l / h$, a flow rate low enough to warrant that no residual oil was left in the injection line \#1. Another reason for this flow rate was also to leave a large amount of oil in the porous device for the recovery tests. Ideally, at the end of this step, one can see that a preferential path was established by the aqueous phase toward the device exit. After 8 to 10 porous volumes of aqueous phase injected at 100 $\mu l / h$, a CLSM image was obtained and the flow rate increased to $1000 \mu l / h$. After the injection of 8 to 10 porous volumes at this higher flow rate, another image acquisition was performed. This was done because, if a high initial flow rate would sweep most of the oil, keeping the initial small flow rate would hinder the penetration of the capsules into the device in the next phase of the experiment. The amount of porous volumes injected in each sweep was chosen so to guarantee that steady state was achieved. This was verified by the fact that no more oil was seen being recovered from the porous device after each sweep.

Diagrams explaining the start of the aqueous phase injection and after the steady state was established are shown in Fig. 3.20 and Fig. 3.21, respectively. 


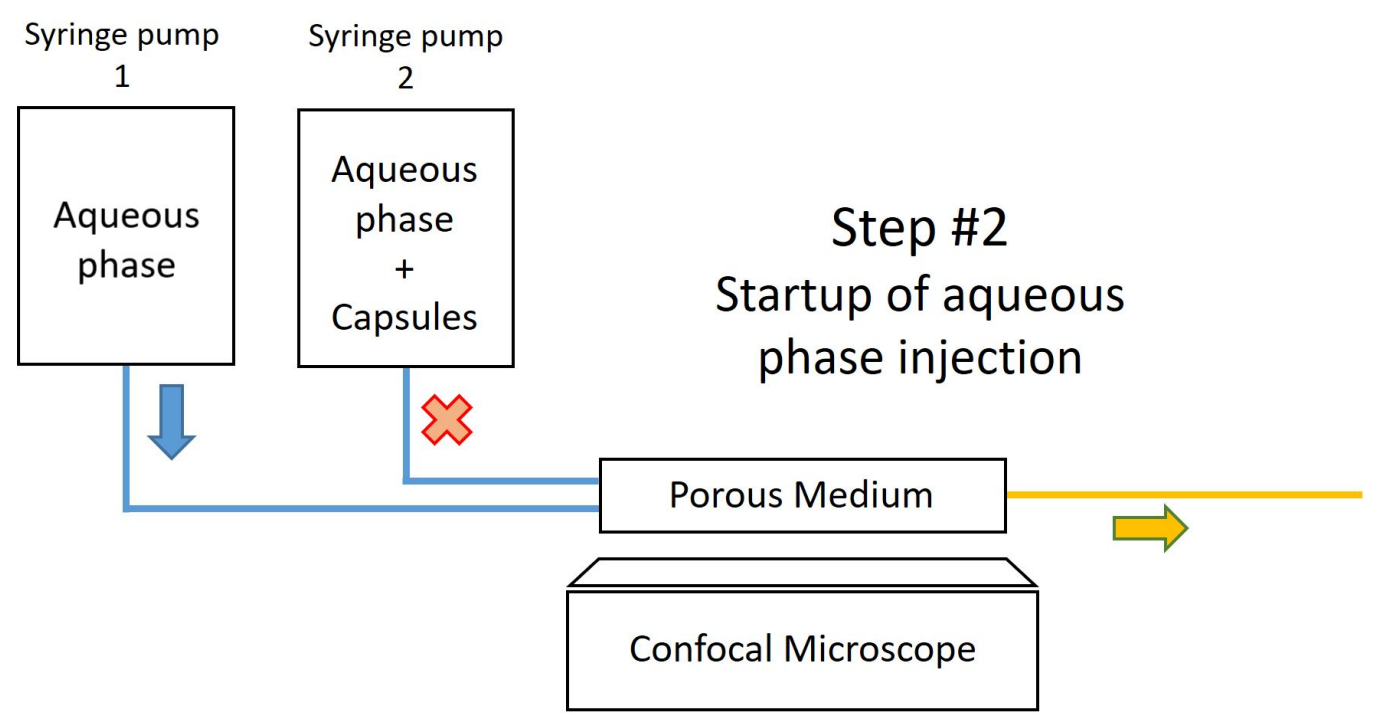

Figure 3.20: Diagram of the startup of aqueous phase injection in the porous device. Oil is being recovered from the device. The line filled with oil phase is shown in yellow and the line with aqueous phase is shown in blue.

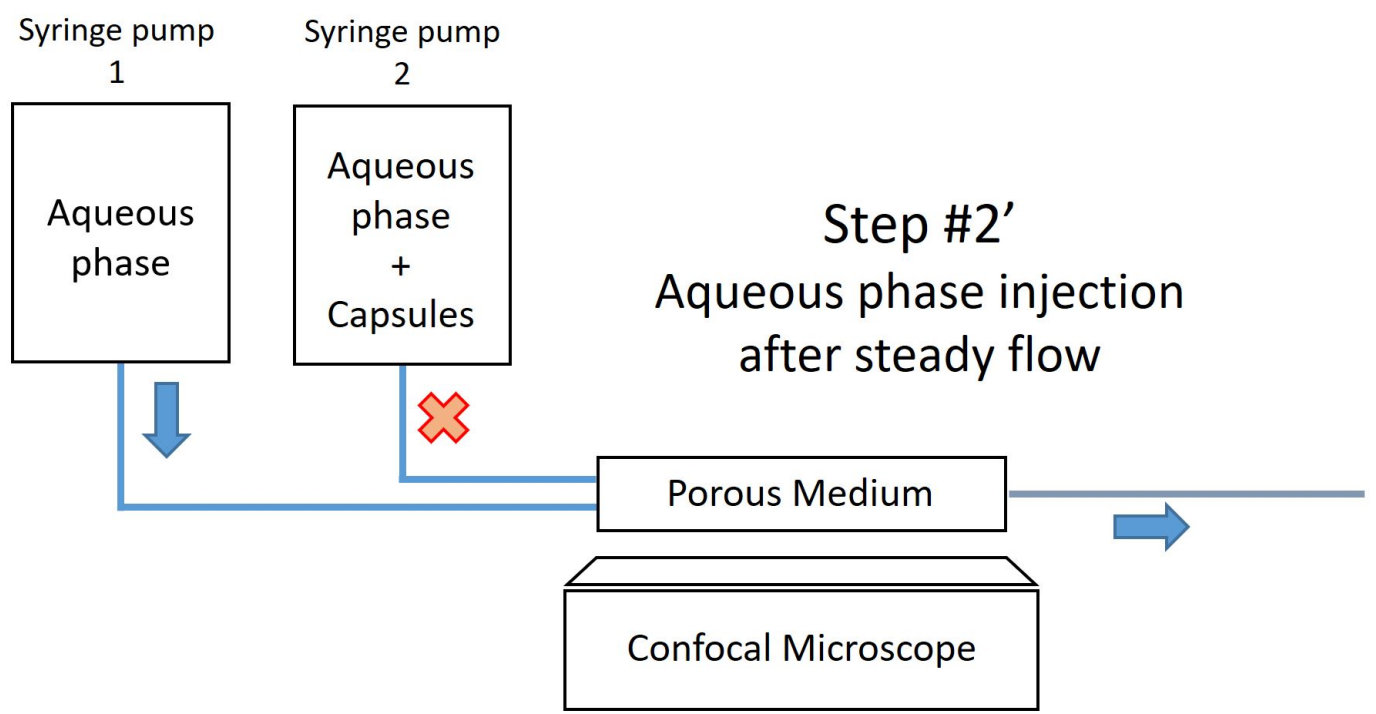

Figure 3.21: Diagram of aqueous phase injection in the porous device after steady state was established. Only aqueous phase is being recovered from the device. The line filled with oil phase is shown in yellow and the line with aqueous phase is shown in blue.

It is important to notice that all the injections were performed after removing the device holder from the microscope and placing it on vertical position, to ensure that the density differences were not influencing the experiment. After reaching steady state, the porous device was placed horizontally again for the image acquisition at the confocal microscope.

After the image of aqueous phase injection at $1000 \mu l / h$ was acquired, the injection of the microcapsules was initiated. A diagram of the experimental 
setup during this phase is shown in Fig. 3.22. The injection from the syringe pump \#1 was stopped, the binder clip that was closing the flow on the tube \#2 was moved to the tube \#1 and the syringe pump \#2 was started with a flow rate of $1000 \mu l / h$. It was possible to observe at this step a volume of oil being displaced from the device, as shown in Fig. 3.22.

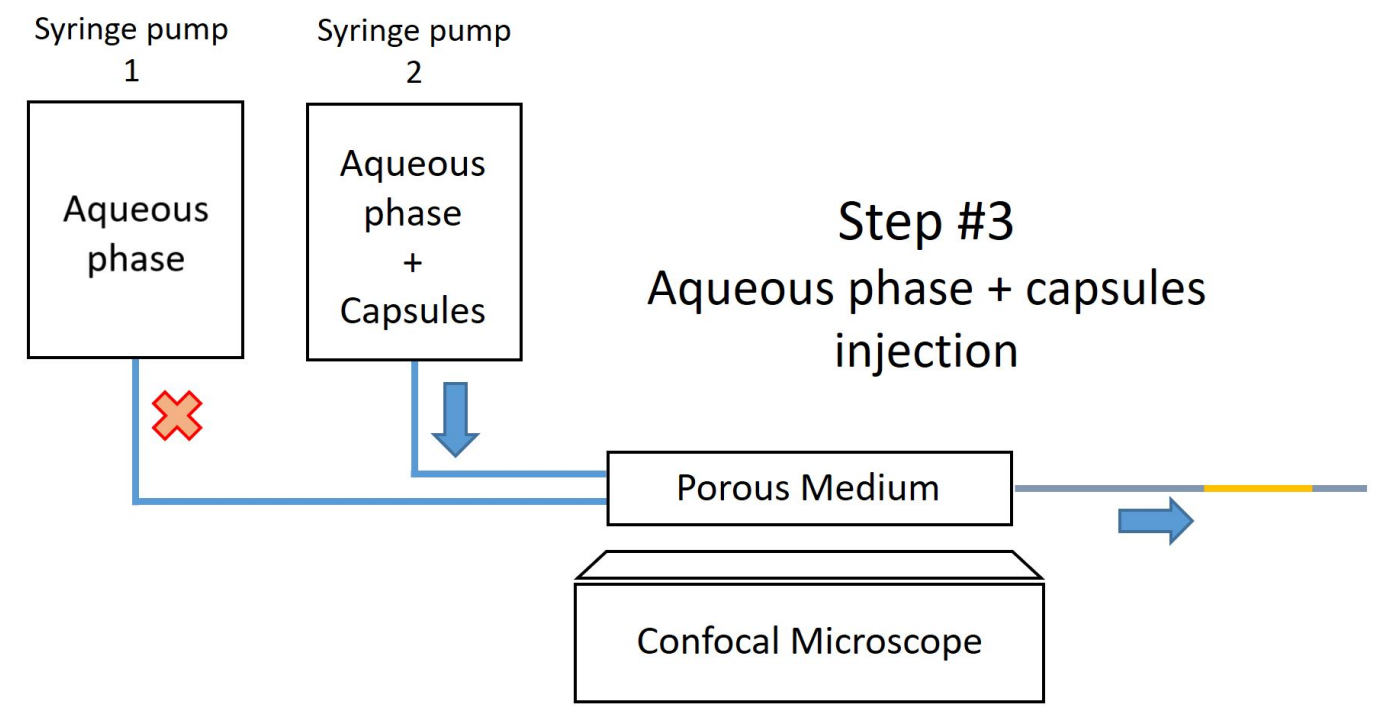

Figure 3.22: Diagram of aqueous phase with capsules injection on the porous medium. Additional oil phase was recovered from the device. The line filled with oil phase is shown in yellow and the line with aqueous phase is shown in blue.

As described in this section, it was possible to fairly reproduce some procedures commonly used by the industry in oil recovery application. For example, future works could use the technique described here to analyze in more details only the effects of water injection.

\subsection{3}

\section{Microscope settings}

In this section the microscopy procedures and the acquisition software setup will be explained. First, to start the acquisition, the microscope, the scanner power and the laser power were turned on, in this order. Then, after initializing the computer and the software LAS X, the use of resonant scanner option was activated.

This option, when activated, controls the way the microscope sweeps the focal plane by rotating mirrors at a specific frequency, thus, collecting pixels at a very high speed. The scanner used has the frequency of 12,000 $\mathrm{Hz}$ and, using also the bidirectional scanning, the acquisition speed was increased to 24,000 lines per second. So, a frame of the focal plane (named a step) with 
512 lines only needs about 20 milliseconds. The bidirectional scanning makes acquisition faster, but it requires a manual calibration to be properly used.

Once the software was opened, some tools and parameters common to all experiments were selected. The objective lens chosen was a PL FLUOTAR with magnification of $10 \times$ and an optical aperture of 0.30 . The lens used was dry, this means it works without an immersion fluid.

For the CLSM technique the image is formed in a sequence of acquisitions, as shown in Fig. 3.23. The full image is divided in tiles, and the tiles are composed by a collection of steps at different focal planes along the tile. Stacking the 2D images, one obtains the tiles, and merging (or stitching) these tiles it is possible to recover a $3 \mathrm{D}$ representative region of the sample.

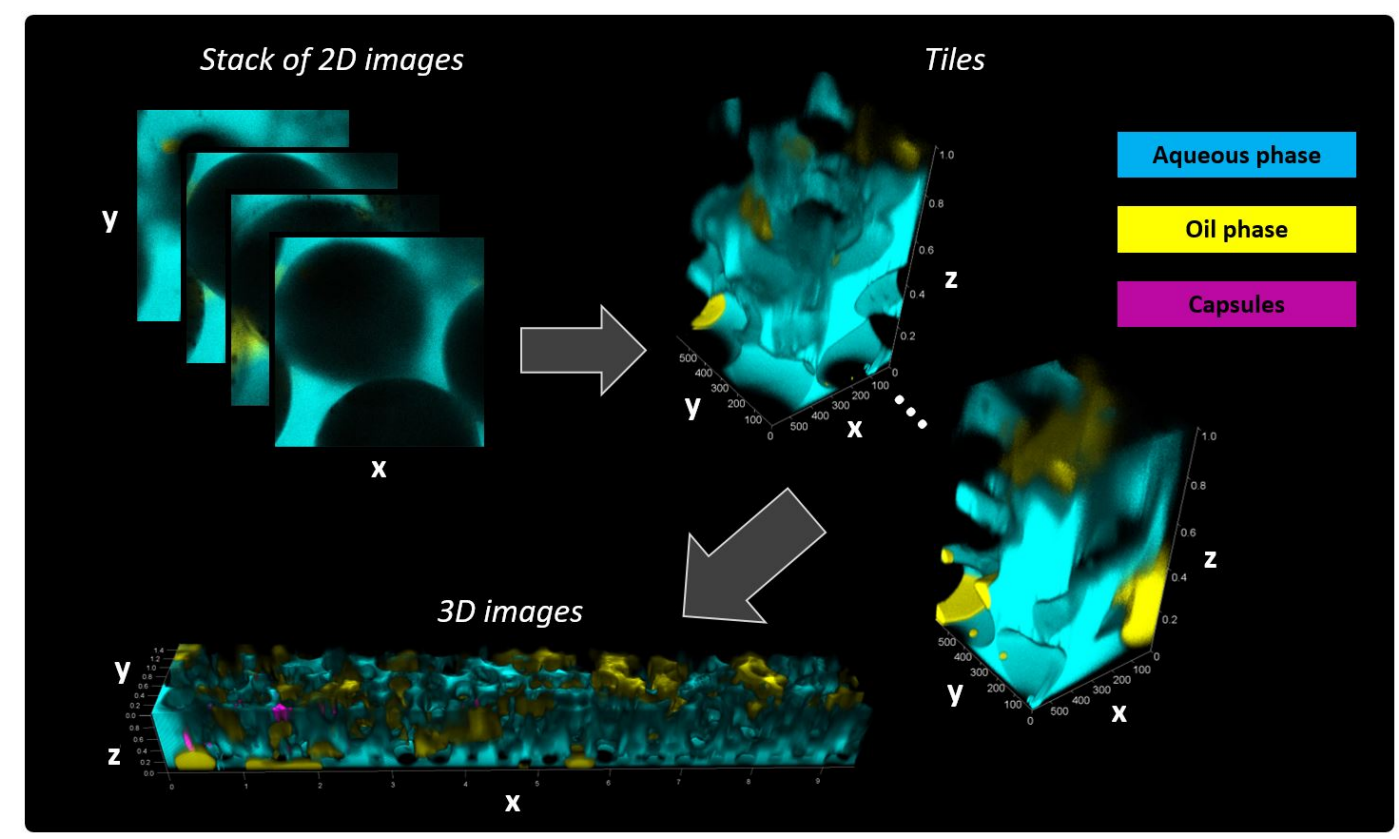

Figure 3.23: Image formation procedure for the confocal laser scanning microscopy technique. The aqueous phase is shown in blue, the oil phase in yellow and the microcapsules in purple.

In this work, the full image was composed by a total of 100 tiles, organized in a mesh of 25 tiles in $\mathrm{X}$ direction and 4 tiles in $\mathrm{Y}$ direction. The mesh position in relation to the porous device used in this work was to place it at the entrance of the porous medium, where the microcapsules injection effects would be easier to observe. The tiles were formed by a stack of 228 steps with a distance of $4.283 \mu \mathrm{m}$ between them, making the tile with a depth of $972.32 \mu \mathrm{m}$. Each step has $512 \times 512$ pixels, that with the objective lens used correspond to a square sized $581.25 \times 581.25 \mu \mathrm{m}$. This makes a pixel resolution of $1.136 \times 1.136 \mu \mathrm{m}$. Thus, the measures of a tile was $581.25 \times 581.25 \times 972.32 \mu \mathrm{m}$.

For faster acquisition, the zoom factor used was the minimum allowed for the system, a factor of 2 . In order to have a sharper image, it was decided 
to set the scanning so that each line in the $\mathrm{X}$ direction was acquired 6 times, and a mean value for each pixel was adopted. The line accumulation parameter used was 1, since the intensity of the image was good enough. Frame average and frame accumulation are similar controls for averaging and accumulating values for pixels, but they were not needed in this work. The pinhole diameter used was $70.72 \mu \mathrm{m}$.

A view of the microscope control panels with these settings is shown in Fig 3.24.
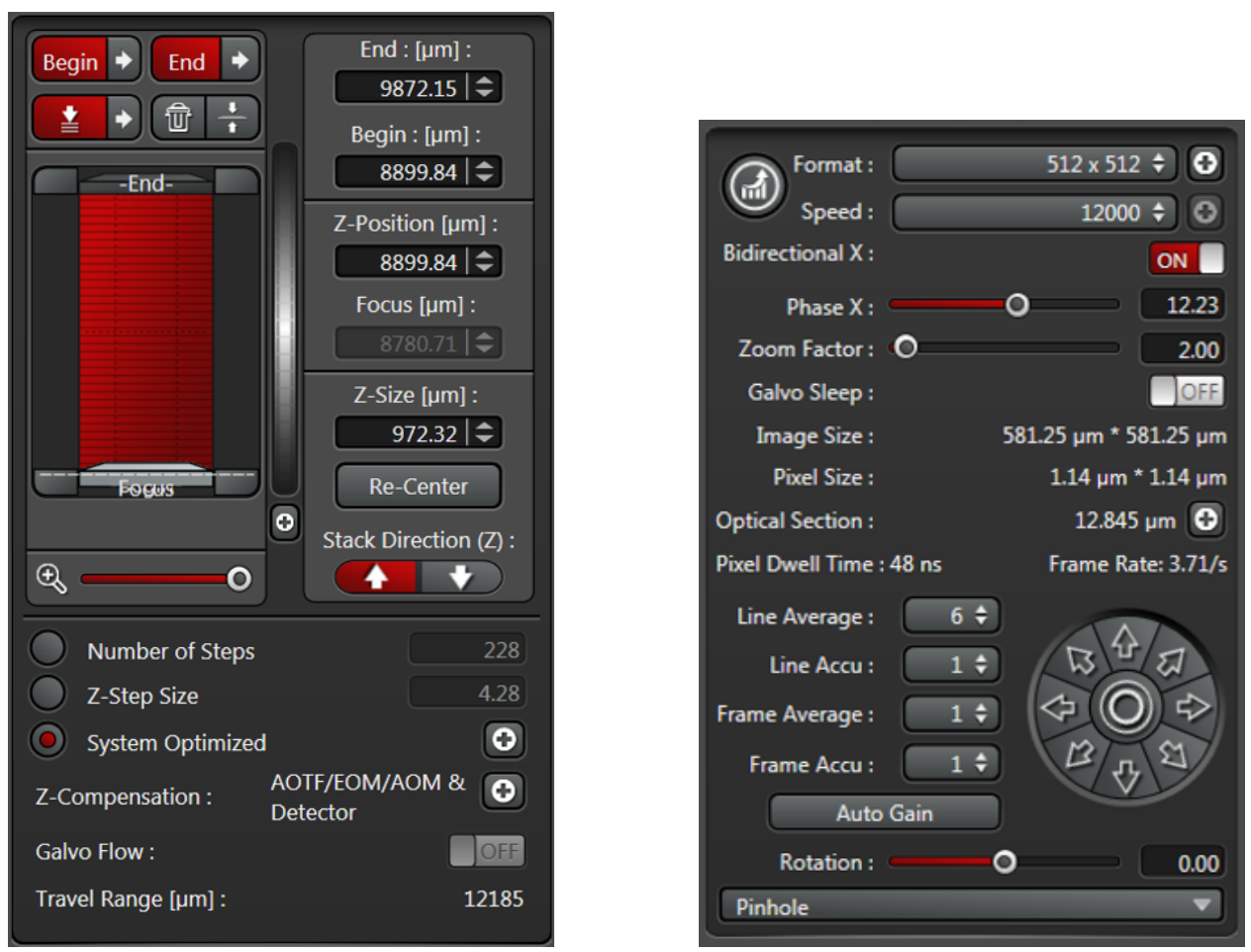

Figure 3.24: Software settings for the image acquisition.

Merging the tiles is a delicate operation. To perform it some overlap between adjacent tiles is necessary. Larger overlaps lead to a more precise reconstruction of the 3D image, however they may consume more time to obtain the same given region. After some preliminary tests, it was found that values for overlaps of $20 \%$ in the $\mathrm{X}$ direction and of $30 \%$ in Y direction would allow a good compromise between 3D image quality (without discontinuities) and acquisition time. The software allows also to set the speed and accuracy of the numerical stitching. In order to get better results, it was chosen to perform it with the lowest speed and a the finest accuracy.

As noticed in Fluorescent pigments section, 3.1.3.2, three lasers were used in this work: the $488 \mathrm{~nm}$ laser was used for the excitation of the oil phase, the $552 \mathrm{~nm}$ laser for the microcapsules and the $638 \mathrm{~nm}$ for the aqueous phase. Two sequential acquisitions were needed to visualize the three phases, this occurred 
because the number of phases was higher than the number of detectors (3 phases for 2 detectors). The type of sequential scan selected was to alternate the acquisition between stacks, that way was possible to select different laser intensities for each sequential step. The confocal microscope was equipped with one photomultiplier tube (PMT) detector and one hybrid detector (HyD). Both of these sensors convert photons to photoelectrons. For the first sequential step (acquisition of oil and aqueous phase) the range of detection used by each one of the detectors were $493 \mathrm{~nm}$ to $545 \mathrm{~nm}$ for the oil phase using the PMT detector and $660 \mathrm{~nm}$ to $700 \mathrm{~nm}$ for the aqueous phase using the HyD detector. The selection of the range for each detector is shown in Fig. 3.25. The second sequential step (in which the microcapsules were mapped) was set using only the PMT sensor in the range of 562 to $630 \mathrm{~nm}$.

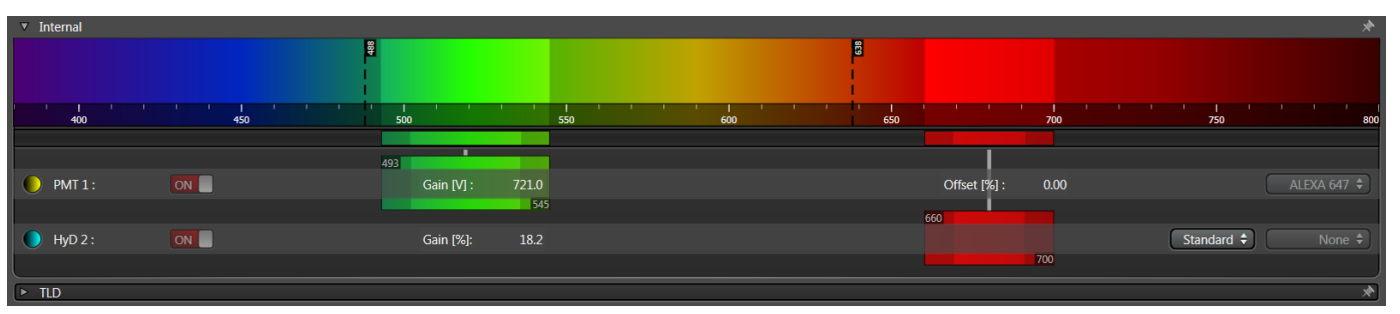

Figure 3.25: Selection of the range of acquisition for each detector (PMT and $\mathrm{HyD}$ ) in the first sequential step. The oil phase was acquired using the PMT detector and the aqueous phase using the HyD detector.

Unfortunately, using this sequential setup, the detector range used for the oil phase also mapped the microcapsules, as shown in Fig. 3.26.
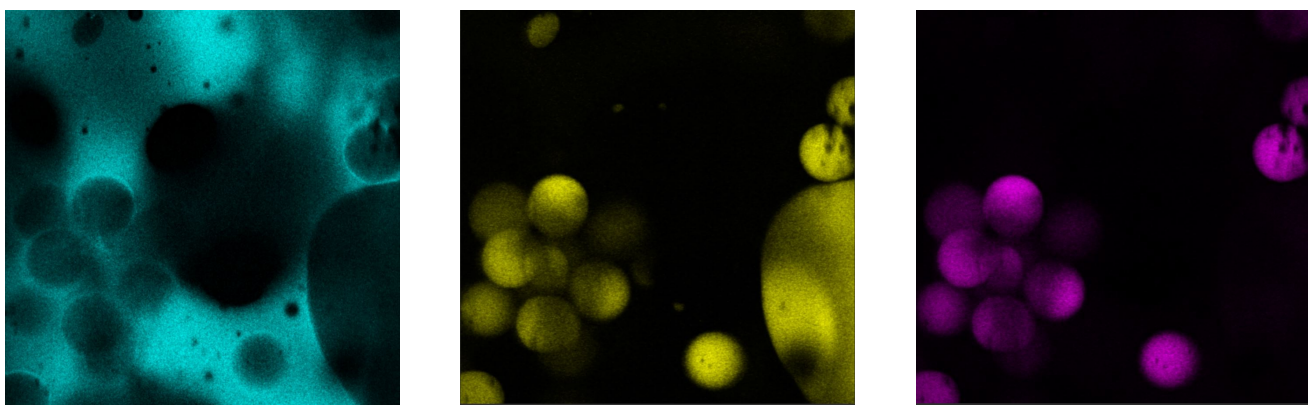

Figure 3.26: Example of images from the aqueous phase detector channel, oil phase detector channel and microcapsules detector channel. The aqueous phase detected in the first image has lower intensity in the region occupied by the oil phase and microcapsules. The oil phase detects the fluorescence from the oil phase and from the microcapsules. The last image is from the microcapsules channel, in which only the microcapsules are detected.

Probably the cause of the simultaneous oil phase and capsules detection at the PMT detector in the first sequence is due a small excitation of the fluorescent pigments inside the microcapsules by the $638 \mathrm{~nm}$ laser. The evidence 
is that in the previous test, comparing only the oil phase with the capsules without the aqueous phase visualization, the distinction was performed in a perfect way.

This problem was solved once the second acquisition sequence did not acquire the oil phase, so it allowed to subtract the data in order to quantify. For the qualitative visual comparison the subtraction was not needed, as each phase was simultaneously view, was possible to distinguish the colors from the capsules (yellow + purple) and the oil ganglia (yellow).

An important software tool implemented in this work was the zcompensation. It allowed setting a linear increase for the detector gain and for the laser intensity in deeper focal planes. By increasing the laser intensity, it was possible to excite focal planes that were not reached by the laser with lower intensity (ideal for the focal planes closer to the device wall). This tool was used by defining the laser intensities and the detectors gain for the first focal plane, z-position equals to 0 , and for the deepest focal plane, z-position equal to $972.32 \mu \mathrm{m}$. The software then interpolates the laser intensity for focal planes bound by these two. In every experiment the reference position was set on the first focal plane, positioned in between the device wall and the plane that passes through the glass beads center.

The microscope control panel setup for the first sequential acquisition is presented on Fig. 3.27. The $488 \mathrm{~nm}$ laser started at $30 \%$ and went up to $70 \%$, the $638 \mathrm{~nm}$ laser started at $4 \%$ and increased up to $40 \%$. The PMT gain increased from $585 \mathrm{~V}$ to $750 \mathrm{~V}$ and the $\mathrm{HyD}$ from $10 \%$ to $20 \%$. For the microcapsules acquisition (not shown in the figure), the z-compensation varied the $552 \mathrm{~nm}$ laser from $30 \%$ to $70 \%$ and the detector gain from $585 \mathrm{~V}$ to $750 \mathrm{~V}$. 


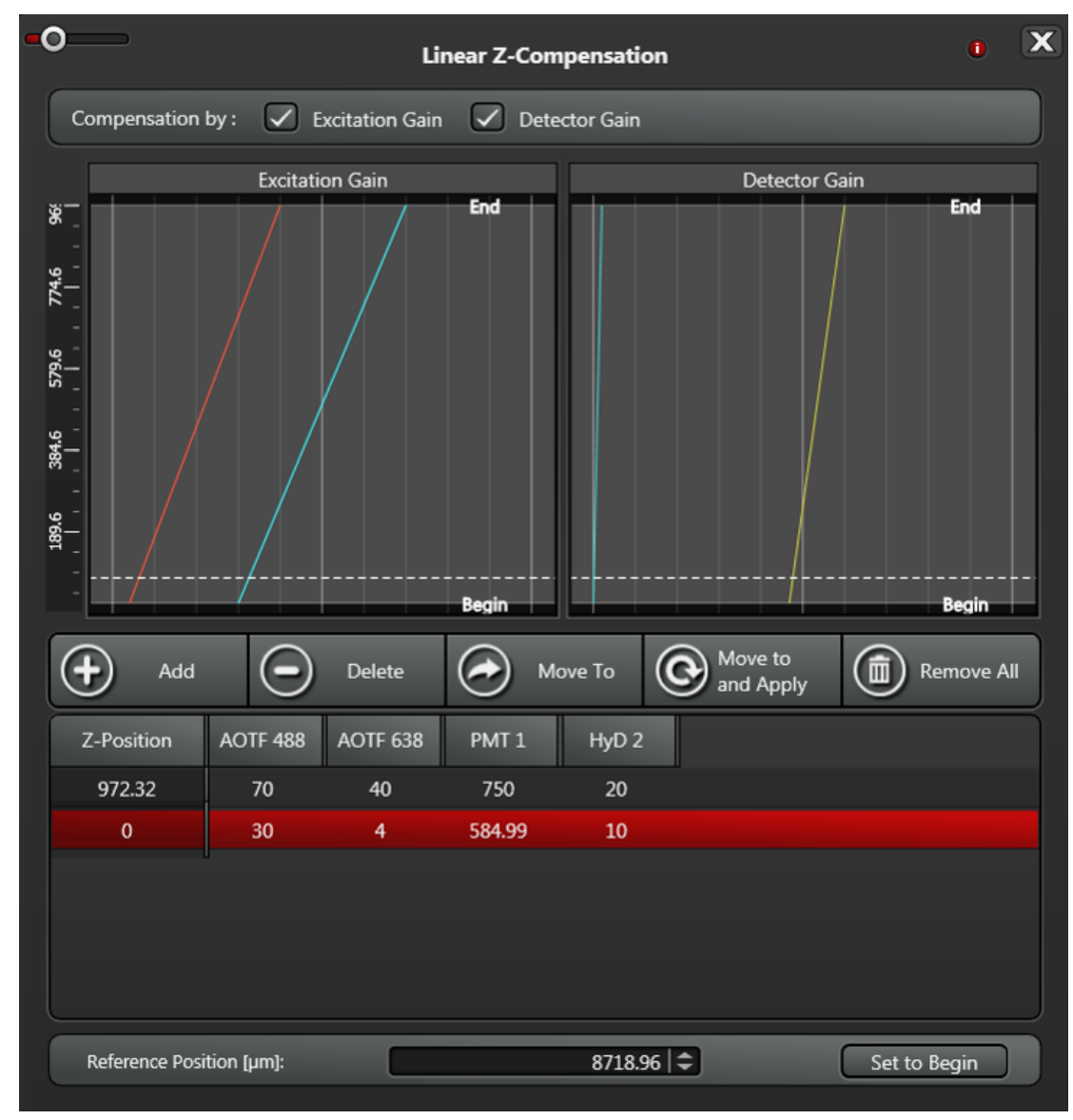

Figure 3.27: Linear z-Compensation setting window for the first sequential step.

The software time estimation for image acquisition in experiments with only one sequential step was of 1 hour, 25 minutes and 47 seconds. For the 3D images that needed two sequential steps, the estimation was 3 hours, 52 minutes and 24 seconds. Normally, the microscope took, on average, 20 minutes longer than this estimation to finish the merging process.

\section{3}

\section{Data processing}

The last part of the experimental procedure was to process the data acquired in order to perform image comparisons, quantitative analysis and reach conclusions from the experimental results.

Concerning the data processing, the first thing was to export the image files from the acquisition software. Once the file .lif (Leica Image File) with the desired image was opened on LAS X it was selected the option to export the file as .tif (Tagged Image File Format) whitout overlaying channels and saving in multi page. The option of lossless compression and to save RAW data 
was also checked. By that, it was possible to organize the different phases from each step of the experiment in labeled files.

After that, the image processing could be divided in four steps: preprocess, segmentation, post-process and data acquisition. The pre-process, segmentation and post-process steps were performed using the ImageJ software. The macro developed is shown in Fig. 3.28.

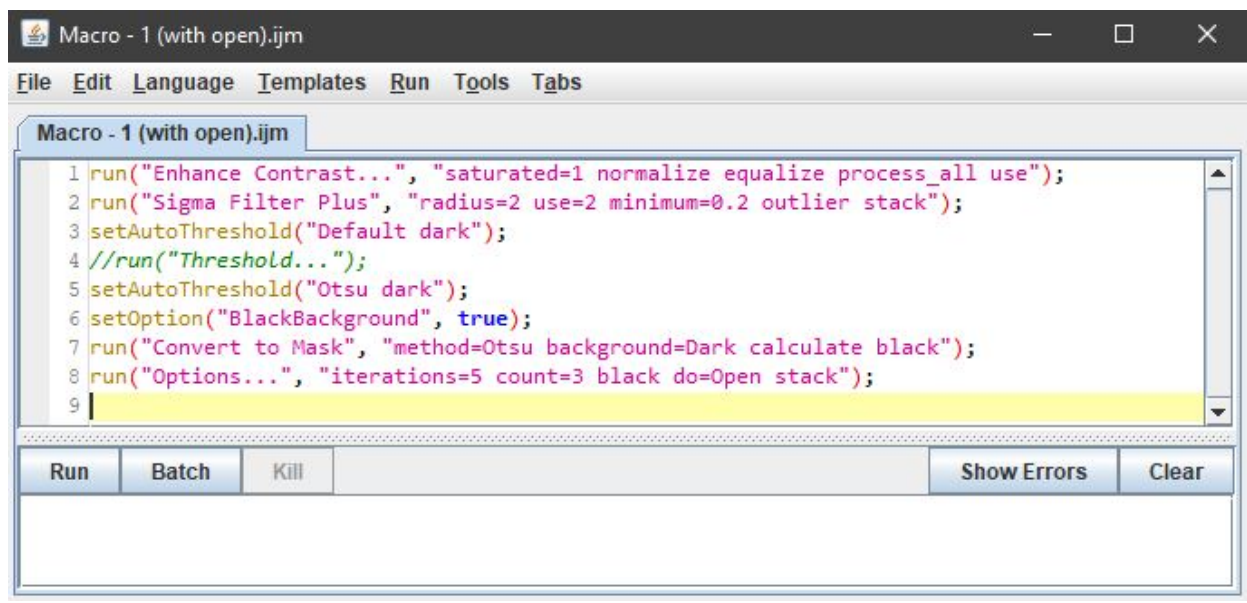

Figure 3.28: Macro file developed in ImageJ.

The data acquisition was performed using the Dragonfly software and will be described further in this section.

\subsection{1 \\ Pre-processing}

The pre-processing could be divided in two operations: the enhance contrast and the filtering. These operations are ordered in the line 1 and 2 of the Fig. 3.28.

The enhance contrast was performed to equalize the histogram of each 2D image. The saturated pixels determines the percentage of pixels in the image that are allowed to become saturated.

For the filtering, it was chosen the Sigma filter. The Sigma filter was the one that best fit for the images obtained by the experiments described in this work. Some test were performed using the Non-Local-Means filter instead, but it took much more time and the result was similar to the Sigma filter. A work done by Lee [48] compares the use of the Sigma filter with commonly used median filter for image smoothing. 


\subsection{2}

\section{Segmentation}

As the 3D images produced on this work were a stack of 228 2D images, it was important to use an automatic segmentation method, so it would take less time and would remove the operator influence on the threshold value selection. The segmentation part of the macro is managed by lines 3 to 7 in the macro shown in Fig. 3.28.

A survey on different automatic methods was performed and then the images were tested. The results showed that the Otsu method worked well for images that presented broader histogram, but for darker and with less information images (normally from deeper planes) it presented some imprecision. In those cases, the Yen method fitted better than the Otsu, as shown in Fig. 3.29. In Fig. 3.29 the original image has lower pixel intensities than average images, leading to a misselection of the oil in the region marked by the red circle. In this case the Yen method presented a better result.
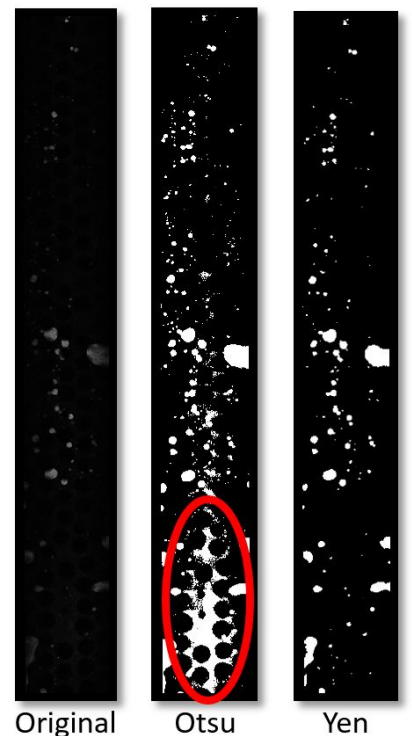

Figure 3.29: Residual oil inside the device after the injection of the aqueous phase. Comparison from the Otsu and Yen method for images with low pixel intensities and small amount of information.

Another way to solve the misselection problem was to add a subtraction between the phases after the post-process, instead of varying the automatic segmentation method. By doing so, a pixel that holds overlap information from different phases would be assigned to store only one of them. Using this methodology, the false remaining oil shown inside Fig. 3.29 red circle would be subtracted by the aqueous phase and as result the misselected region would be corrected. 


\subsection{3}

\section{Post-processing}

The last line of the macro used, Fig. 3.28, was responsible for the postprocessing of the image. The post-process means to apply some operations after the segmentation and, in this work, only the open operation was used. The open operation performs an erosion, followed by a dilation. This smooths objects and removes isolated pixels. The window where the parameters were set is shown in Fig. 3.30.

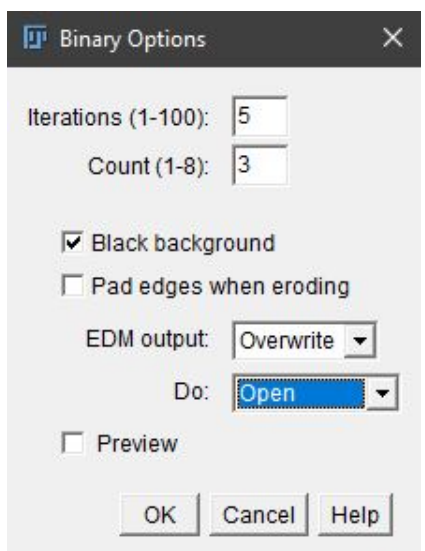

Figure 3.30: ImageJ window to apply the configurations for the open operation.

In the above figure, iterations specifies the number of times opening is performed, count specifies the number of adjacent background pixels necessary before a pixel is removed from the edge of an object during erosion and the number of adjacent foreground pixels necessary before a pixel is added to the edge of an object during dilation.

As described on segmentation section, in order to correct the misselections from auto segmentation methods, after the post-processing the images subtraction was performed. These image files were then saved and opened on the software for the data acquisition, Dragonfly.

\subsection{4}

\section{Data acquisition for volume quantification}

Differently than the other parts, this step of the data processing was done using the software developed by the ORS company called Dragonfly. The Dragonfly software presented a 3D visualization and 3D analysis more complete than the ImageJ. This software, however, is not open source, to use all the tools it disposes it was needed to fill a request for non-commercial licensing.

Once the software was started, the image files had to be loaded. Then, the pixel dimensions and the distance between the planes had to be input. As mentioned before, the voxel size was $1.136 \times 1.136 \times 4.283 \mu \mathrm{m}$. 
After the image loading, a manual segmentation was performed. The reason for this second segmentation process on the image was to make the software recognize it as a binary image. As the imported image was already separated on dark or white pixels, any value for the manual segmentation achieved the same result.

Finally, selecting the image correspondent of each experimental step and each phase channel, the software counted the number of white voxels and presented it as a percentage of the whole image. The software also allows to quantify partial volumes of the whole image, such as a ganglion volume. 


\section{4}

\section{Results and discussions}

This chapter reports the results of this work in two parts: those related to the homogeneous porous media and those related to the stratified porous media. At the end of the homogeneous porous media experiments, an oil recovery efficiency quantification and a preliminary experiment with dynamic visualization of the capsules moving inside the porous media are also presented.

\section{1}

\section{Visual qualitative results with homogeneous porous media}

The homogeneous porous device was the first type of porous medium tested in this work, since it was the easiest to fabricate. So, following the experimental procedure described in the previous chapter the images below were obtained. Important to remember that in all images the color pattern to represent each phase was blue for the aqueous phase, yellow for the oil phase and purple for the microcapsules.

The four homogeneous devices used to obtain these results were named "d", "i", "I" and "II". Many other devices were also fabricated but those were the ones that better suited the experiment. The devices were first identified by letters, but later it was decided to use roman numerals. This was done because of the difficulty to write using a glass marker pen on the small surface of the square capillary.

\subsection{1}

\section{Preliminary results using devices " $d$ " and "i"}

The first two experiments, using devices " $\mathrm{d}$ " and "i" helped to develop the experimental procedure described in the previous chapter. In order to streamline these tests, it was performed the visualization of only one side of the porous medium. The Figures 4.1 to 4.11 are from the device identified as "d" with porosity of $40.4 \%$ and permeability of 193 D.

In Fig. 4.1 it is shown a 3D perspective view of the middle region of the device " $\mathrm{d}$ " saturated with the aqueous phase. At this first step, only aqueous phase was inside the device. 

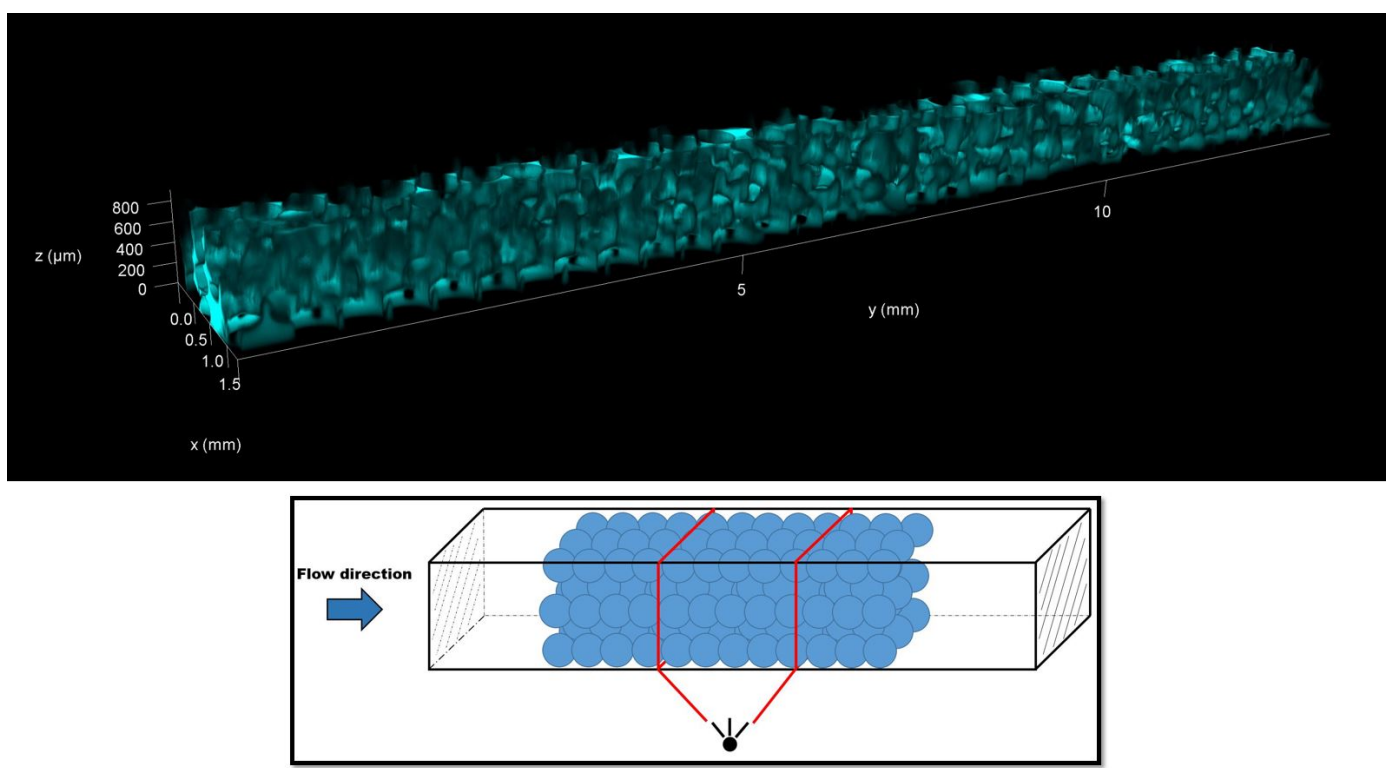

Figure 4.1: 3D perspective view of the middle region, as shown in the sketch, of the device "d" saturated with aqueous phase. Reconstruction formed by the stack of 210 images.

Figure 4.2 is a 2D image of a single focal plane that was used to form the $3 \mathrm{D}$ reconstruction shown in Fig. 4.1. In some cases, to visualize a specific region or the changes between each step of the experiment it is better to analyze the image using the single slices separately. In that way, the images of different depth will not block the visualization.

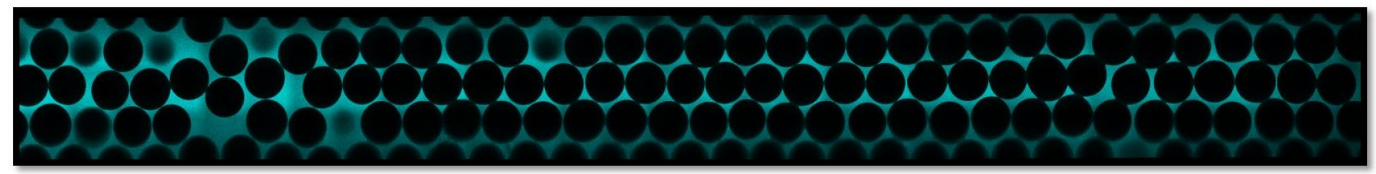

Figure 4.2: Example of 2D image used in the stack to recreate the 3D visualization shown in Fig. 4.1. The dark circles are the glass spheres that don't emit fluorescence and the aqueous phase is represented in blue.

After the saturation of the aqueous phase, the next step was to saturate the device with the oil phase. At this moment the oil phase was not yet changed to Castor Oil, as described in the previous chapter. The 3D perspective view of the device after the oil phase saturation is shown in Fig. 4.3. As the porous medium was previously filled with the wetting aqueous phase, it was expected that some irreducible aqueous phase (connate water) would be observed at this step. 


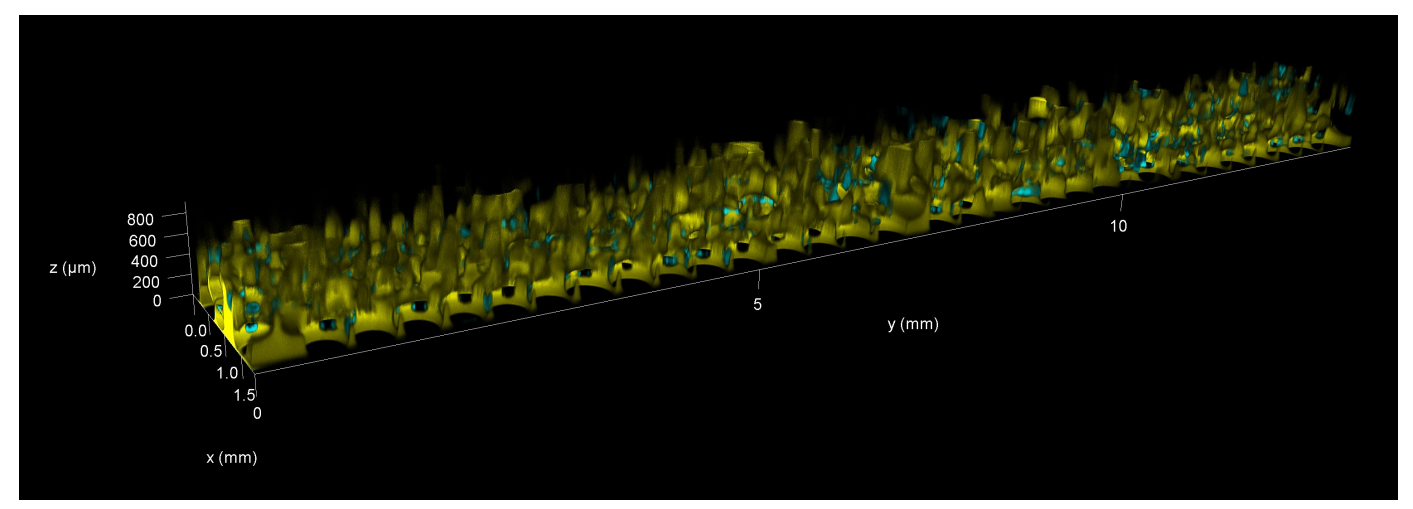

Figure 4.3: 3D perspective view of the device "d" after the oil phase saturation. The oil phase is shown in yellow and the aqueous phase in blue.

In order to make easier the visualization of the irreducible aqueous phase, it is possible to display only one of the phases using the LAS X software. Figure 4.4 shows the same image from Fig. 4.3 but without the oil phase. It is possible to visualize the pattern and the position inside the device of the remaining aqueous phase.

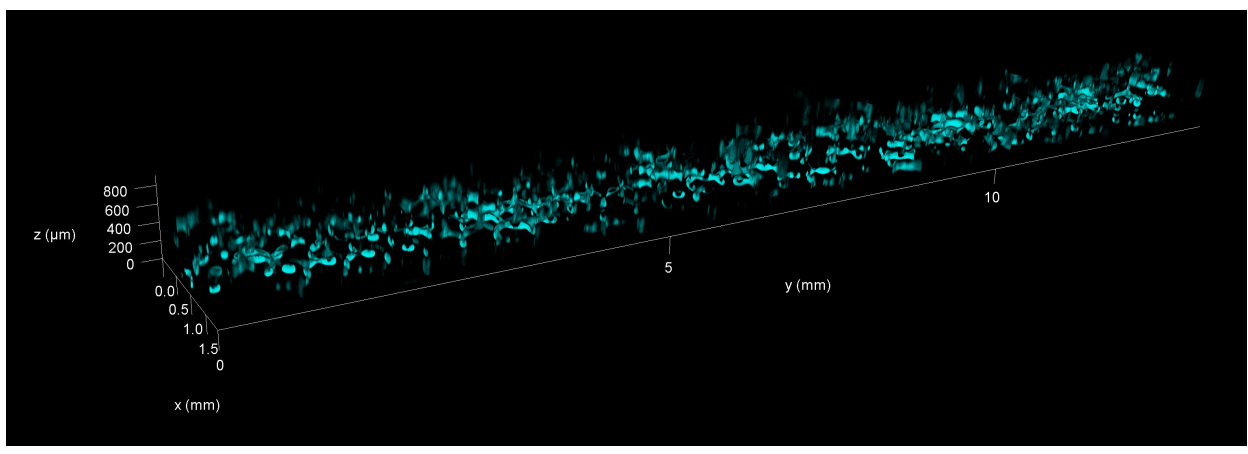

Figure 4.4: 3D perspective view of the device "d" after the oil phase saturation showing (after software manipulation) only the remaining aqueous phase left inside the porous medium, called irreducible aqueous phase.

As explained before, analyzing the 2D images, in some cases, could lead to the observation of interesting effects that would be much more difficult to visualize on the $3 \mathrm{D}$ perspective view. In Figure 4.5 is possible to compare the result after an increase of the oil injection flow rate, from $100 \mu l / h$ to $500 \mu \mathrm{l} / \mathrm{h}$. The red circles highlight regions where the porous medium was filled with aqueous phase and, after the increase of the oil injection, the oil phase displaced the aqueous phase in those regions. 


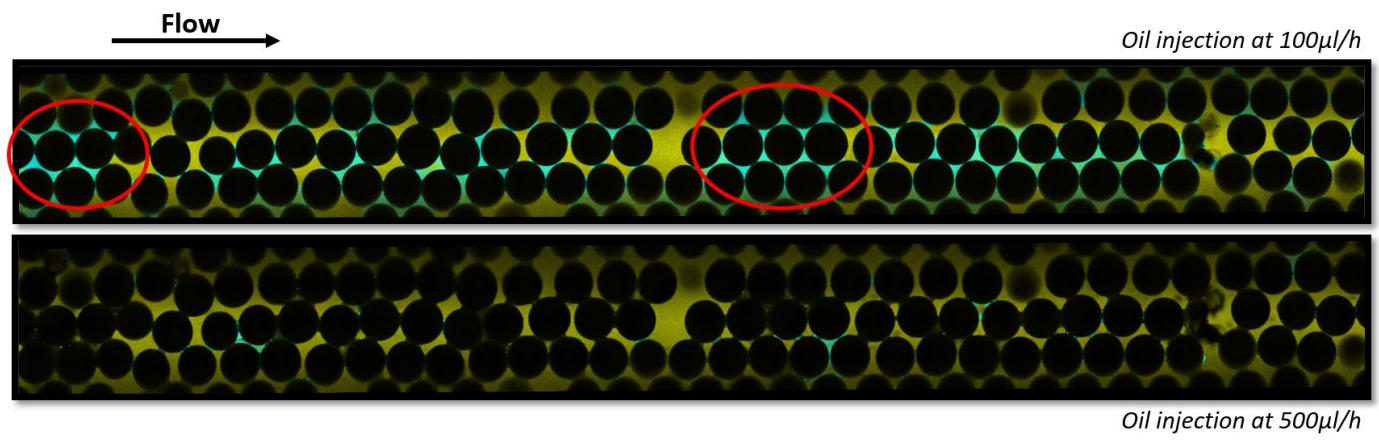

Figure 4.5: Comparison of the oil saturation at different flow rates. The upper image shows the result of a single slice of the 3D image at $100 \mu l / h$ and the bottom image shows the same plane after the increase of the oil phase flow rate to $500 \mu l / h$.

Figure 4.6 shows a view of a single tile, $581.25 \times 581.25 \mu \mathrm{m}$, in which is possible to observe in detail the irreducible aqueous phase wetting the glass spheres after the oil saturation. The picture clearly shows the effects of capillary forces.

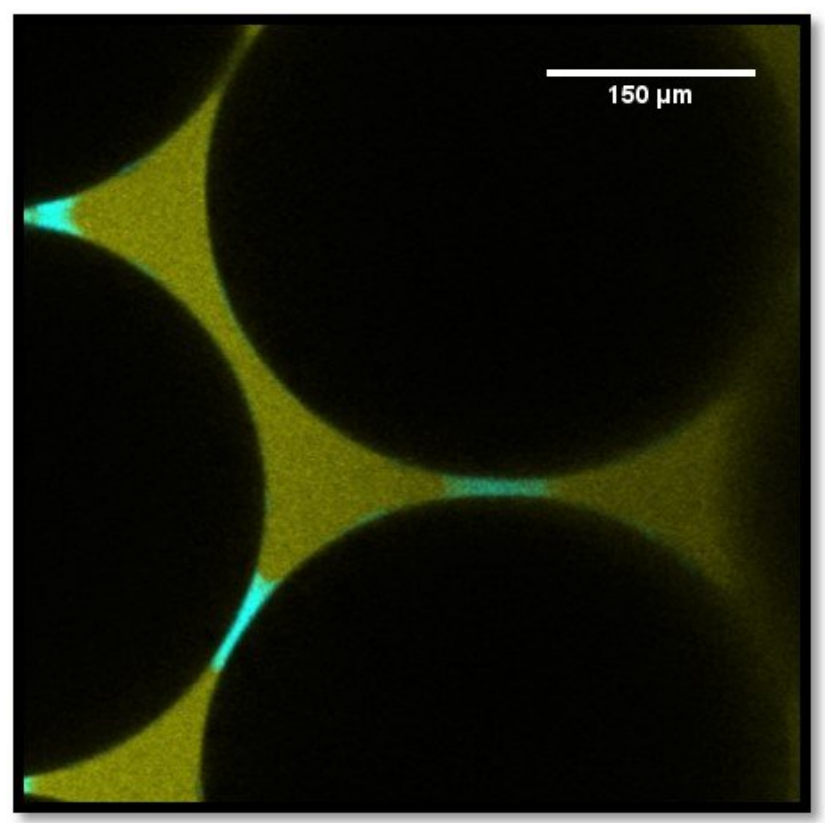

Figure 4.6: Detailed view of a single plane of the device "d" after the oil saturation. It is possible to observe the irreducible aqueous wetting phase in blue and the oil, non wetting phase, in yellow. The visualization area of the image is $581.25 \times 582.25 \mu \mathrm{m}$.

After the oil phase saturation, the aqueous phase injection was performed. In this first experiment it was used the injection flow rate of $250 \mu \mathrm{l} / \mathrm{h}$ $(\approx 5 \mathrm{ft} / \mathrm{day})$. It is possible to observe in Fig. 4.7 that the aqueous phase (blue) displaced a large part of the oil phase (yellow). 


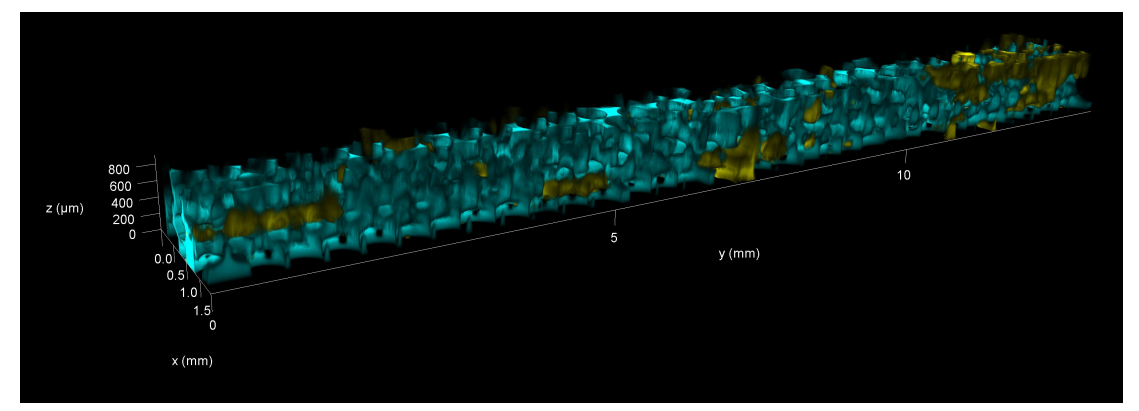

Figure 4.7: 3D perspective view at the middle region of device " $\mathrm{d}$ " after the aqueous phase injection at $250 \mu \mathrm{l} / \mathrm{h}$. The aqueous phase displaced a large part of the oil phase.

After that, for the first time in this work, the microcapsules were injected into the porous device. Initially it was injected a suspension of microcapsules larger than the average pore diameter, with $208 \mu \mathrm{m}$ diameter and $5 \mu \mathrm{m}$ shell thickness. The result, shown in Fig. 4.8, reveals that the microcapsules were not effective displacing the trapped oil. In fact, the microcapsules did not even penetrate into the porous medium. The 3D image obtained was almost identical to the image of the device after the aqueous phase injection.

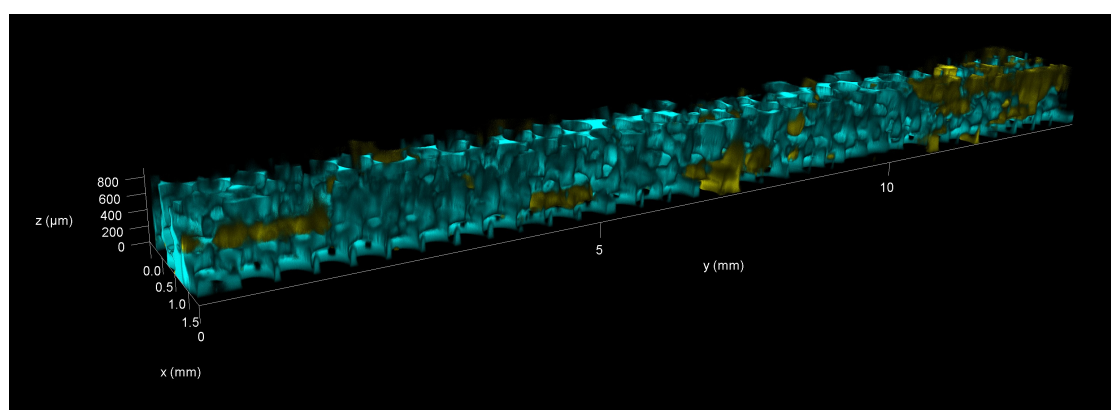

Figure 4.8: 3D perspective view at the middle region of device "d" after large microcapsules injection at $250 \mu \mathrm{l} / \mathrm{h}$. The microcapsules tested in this experiment were not effective to increase the displacement of oil phase. In fact, it was not possible to observe any microcapsules in this region of the device.

In this case, it was possible to conclude from the analysis of single plane images that the oil ganglia inside the porous medium suffered no influence of the microcapsules injection. A comparison of the same $2 \mathrm{D}$ slice, before and after the capsule injection, is shown in Fig. 4.9. This comparison was performed for multiple planes and all of them showed similar results. 


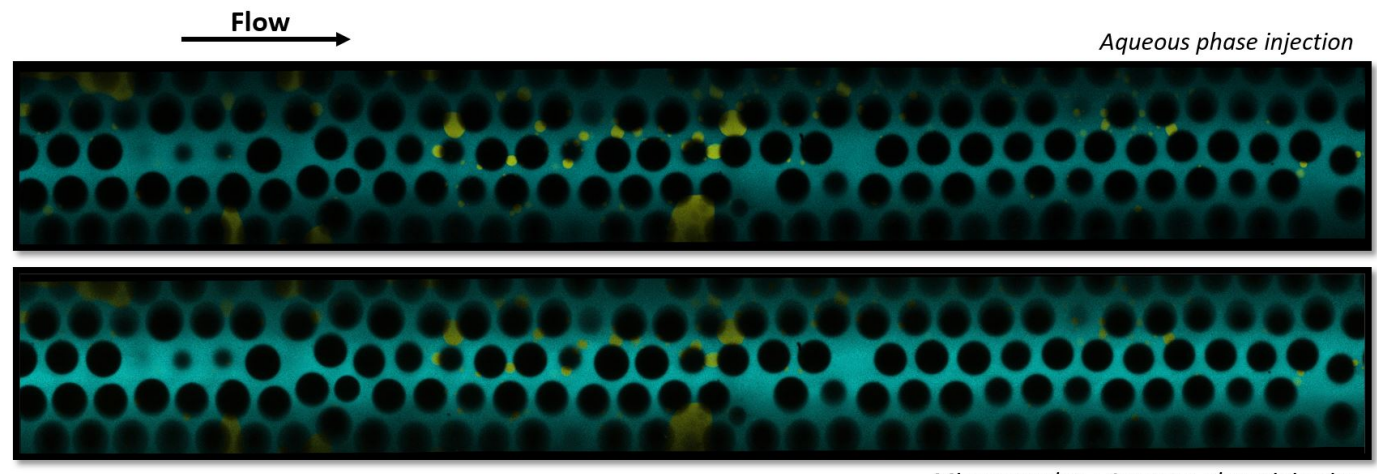

Microcapsules + Aqueous phase injection

Figure 4.9: Comparison between 2D images at the same z-position into the device " $\mathrm{d}$ " before and after the large microcapsules injection. It is possible to notice that the oil ganglia that appear in this plane didn't moved after the microcapsules injection.

It was possible to conclude that the microcapsules did not enter the porous space observing, in Fig. 4.10, an image of a single tile, 581.25 x 582.25 $\mu \mathrm{m}$, at the entrance of the porous medium. The $208 \mu \mathrm{m}$ diameter with $5 \mu \mathrm{m}$ shell thickness capsules got stuck at the entrance. This result was, in some aspects, similar to the results obtained in Vimieiro's work [13].

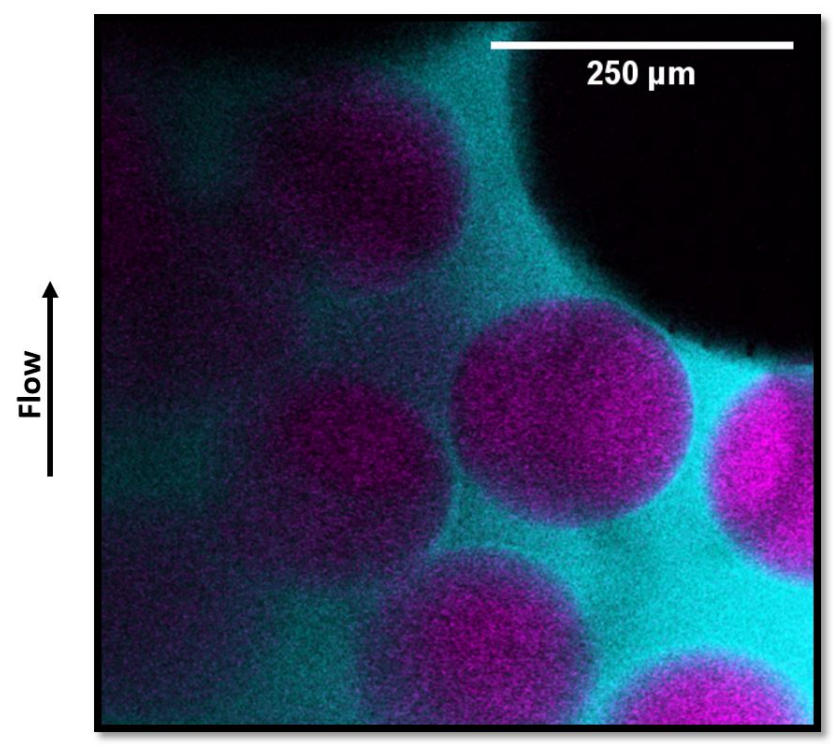

Figure 4.10: Detailed view of a single tile, $581.25 \times 582.25 \mu \mathrm{m}, 2 \mathrm{D}$ image positioned at the entrance of the porous medium. The aqueous phase is represented in blue and the microcapsules are shown in purple. The aqueous phase flow rate was not enough to force the microcapsules inside the porous medium.

After concluding the experiments, to make possible to reuse the devices, they were cleared of the capsules and of the oil phase using high flow rate injections of ultra pure water alternating with air and acetone. 
Once the device "d" was clean, a different type of experiment using it was performed. In order to test a gradual increase in the aqueous phase flow rate, the device was saturated with the oil phase, following the same steps of the previous experiment, and then the aqueous phase injection was applied with sequential increases. Figure 4.11 shows the visualization of the same single plane after different flow rates of the aqueous phase injection.

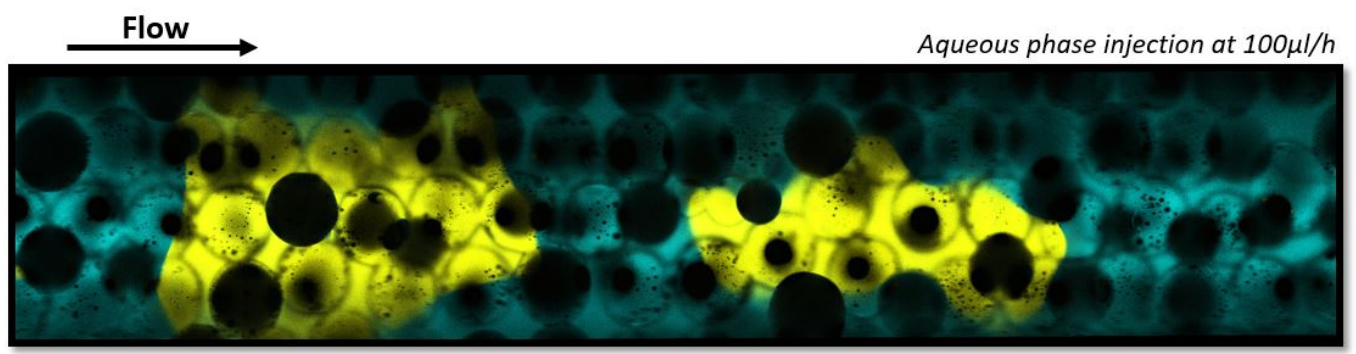

Aqueous phase injection at $1000 \mu \mathrm{l} / \mathrm{h}$

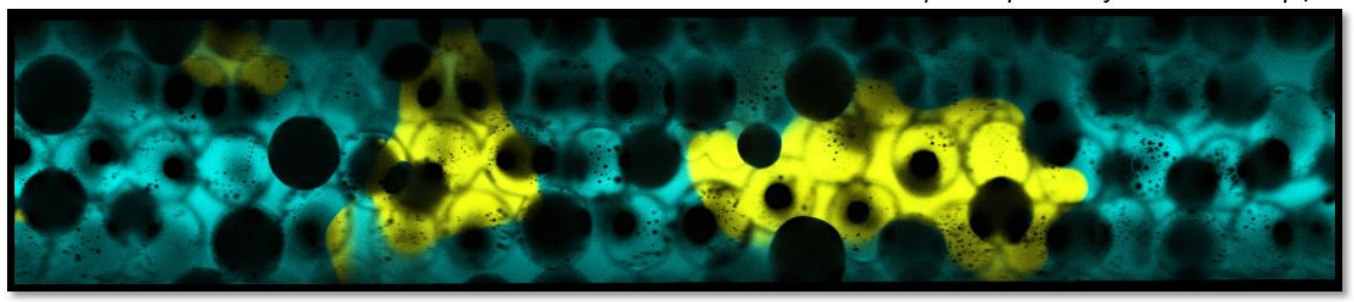

Aqueous phase injection at $2000 \mu \mathrm{l} / \mathrm{h}$

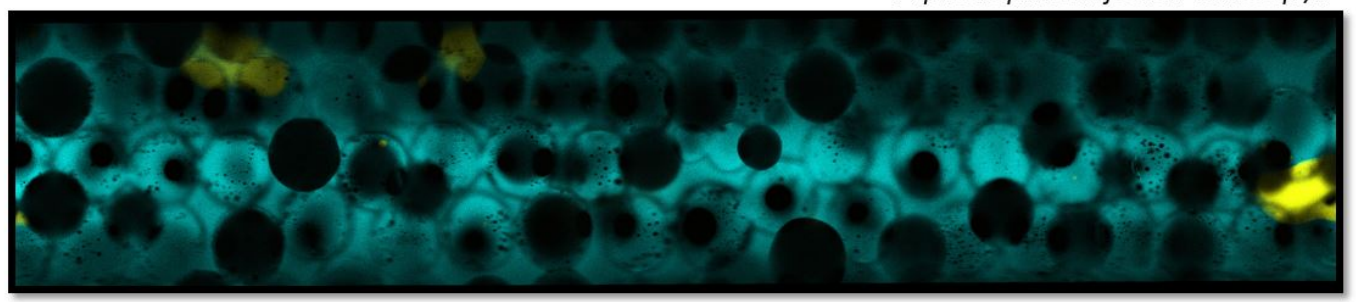

Figure 4.11: Images of the same plane after gradual increases of aqueous phase flow rate injection.

In Figure 4.11, it was possible to observe that after the $100 \mu l / h$ injection two large oil ganglia appeared in this visualized region. After the increase of the flow rate to $1000 \mu l / h$, the oil ganglion closed to the inlet broke in two smaller ganglia. After the increase of the flow rate to $2000 \mu l / h$ almost every residual oil ganglia were recovered.

The values of Capillary Number for the flow rates used to inject the capsules in most experiments were $C a \approx 10^{-4}$. The aqueous phase injection at $1000 \mu l / h$ was equal to $C a=1.07 \times 10^{-4}$.

The use of a lower flow rate to create an aqueous phase preferential path worked in the sense that it made possible to achieve some amount of residual oil after higher flow rates. If applied directly, the flow rate of $1000 \mu \mathrm{l} / \mathrm{h}$ would lead to a complete oil sweep. This step increase of flow rate was adopted into the experimental procedure, as described in the previous chapter. 
Other changes, besides the gradual increase of the aqueous phase flow rate, were the use of smaller capsules and the use of Castor Oil as oil phase. The smaller capsules would facilitate the entrance into the porous medium and the Castor Oil increased the viscosity ratio between fluids, contributing to higher amount of residual oil after aqueous phase injection.

Another preliminary experiment was performed using the device "i", which have the porosity of $41.0 \%$ and the permeability was 198 D. The values of porosity and permeability were similar from the device "d". At this experiments different microscope settings were also tested in order to improve the visualization and data analysis.

Figure 4.12 shows the region of the porous medium at initial state saturated with the aqueous phase.

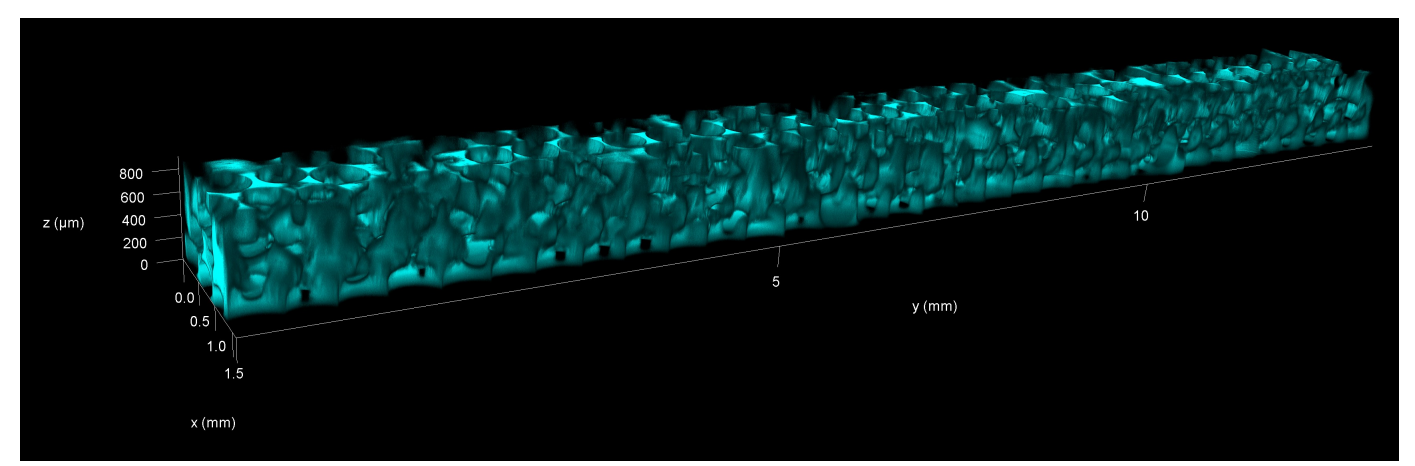

Figure 4.12: 3D perspective view of the middle region of the device "i" saturated with aqueous phase. Reconstruction formed by the stack of 212 images.

Comparing the Figs. 4.1 and 4.12, it is possible to notice that this experiment achieved clearer visualization for deep planes (top part of the porous device in Figs. 4.1 and 4.12). This was possible due the implementation of some tools not used in the previous experiment. The final microscope setup was described at the Microscope settings section, 3.2.3.

Following the aqueous phase saturation, the next experimental procedure step was the oil phase saturation, shown in Fig. 4.13. 


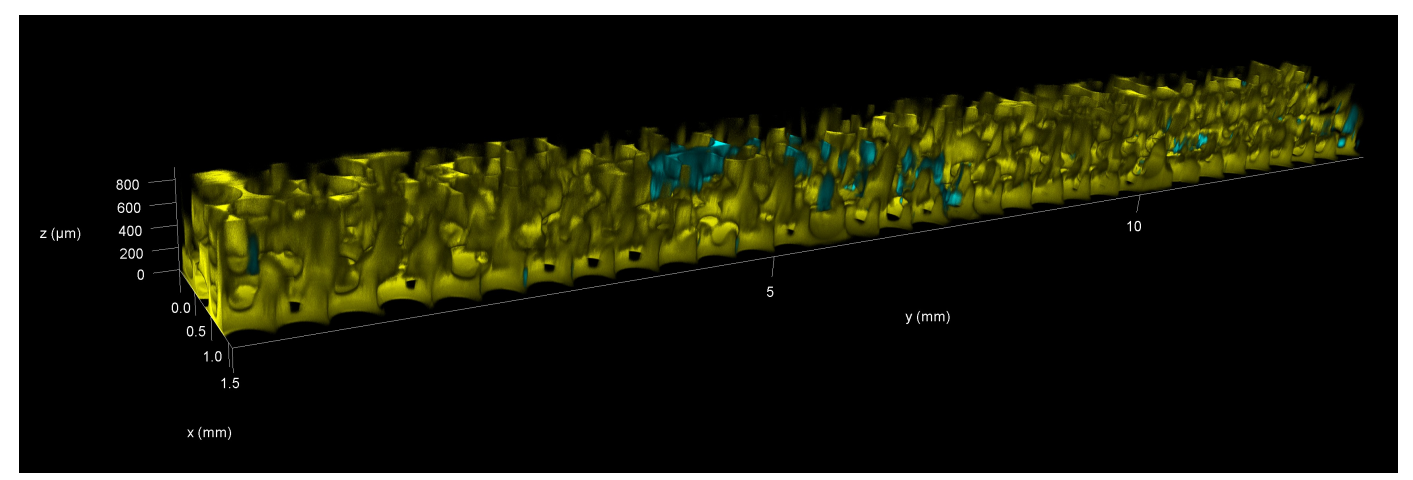

Figure 4.13: 3D perspective view of the middle region of the device "i" saturated with oil phase. Reconstruction formed by the stack of 212 images.

As observed in Fig. 4.13, the amount of irreducible aqueous phase in this experiment was higher than from the previous experiment. It was used a single oil phase flow rate injection that later showed to be insufficient to fully saturate the device with oil. Figure 4.14 shows a detailed view of the region with a large amount of aqueous phase. Despite that, the results achieved after the injection of the aqueous phase and the aqueous phase with the microcapsule suspension were still useful for the preliminary analysis.

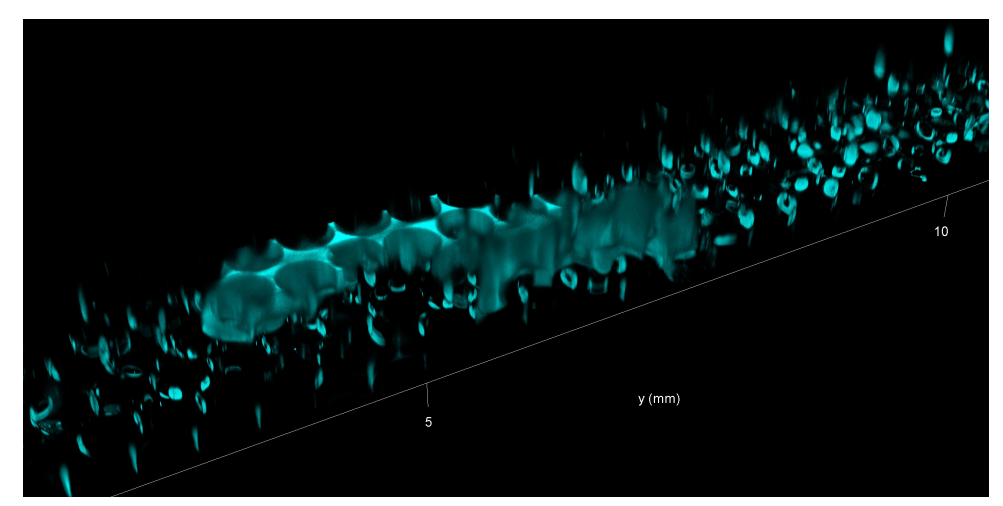

Figure 4.14: Detailed view of the device "i" saturated with oil phase displaying only the aqueous phase.

Then, the aqueous phase gradual injection was performed and the result after $1000 \mu \mathrm{l} / \mathrm{h}$, the final flow rate injection, is shown in Fig. 4.15. 


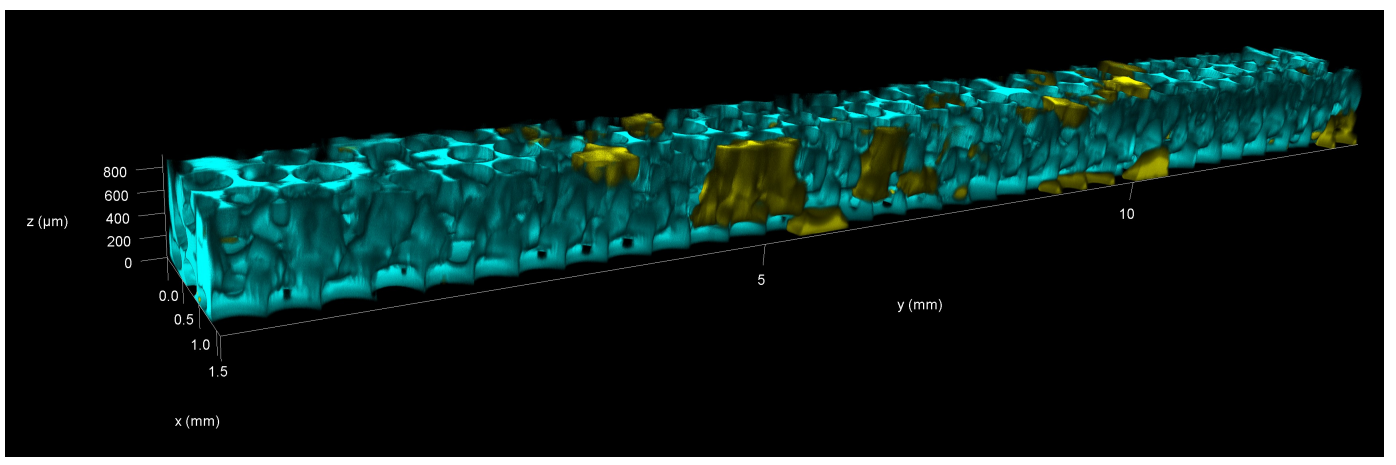

Figure 4.15: 3D perspective view of the middle region of the device " $\mathrm{i}$ " after the aqueous phase injection. Reconstruction formed by the stack of 212 images.

It is possible to see that the aqueous phase displaced a large part of the oil phase and left some oil ganglia trapped in this region of visualization.

So, at this point the microcapsules were injected and the perspective view shown in Fig. 4.16 was acquired.

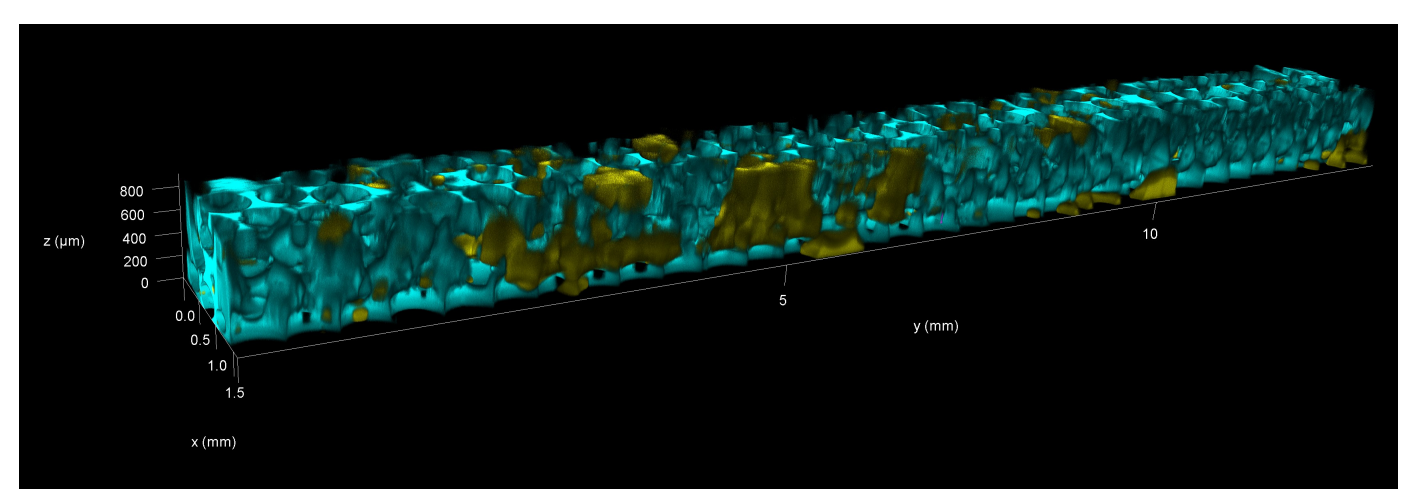

Figure 4.16: 3D perspective view of the middle region of the device "i" after the microcapsules injection. Reconstruction formed by the stack of 212 images.

Comparing the images obtained in this experiment, showed in Fig. 4.15 and 4.16, could lead to a conclusion that more oil phase was found in that same region of visualization after the capsules injection. To analyze that result the same 3D images were observed from the bottom face. The images are compared in Figs. 4.17 and 4.18, where the aqueous phase was hidden for deeper oil phase visualization. 

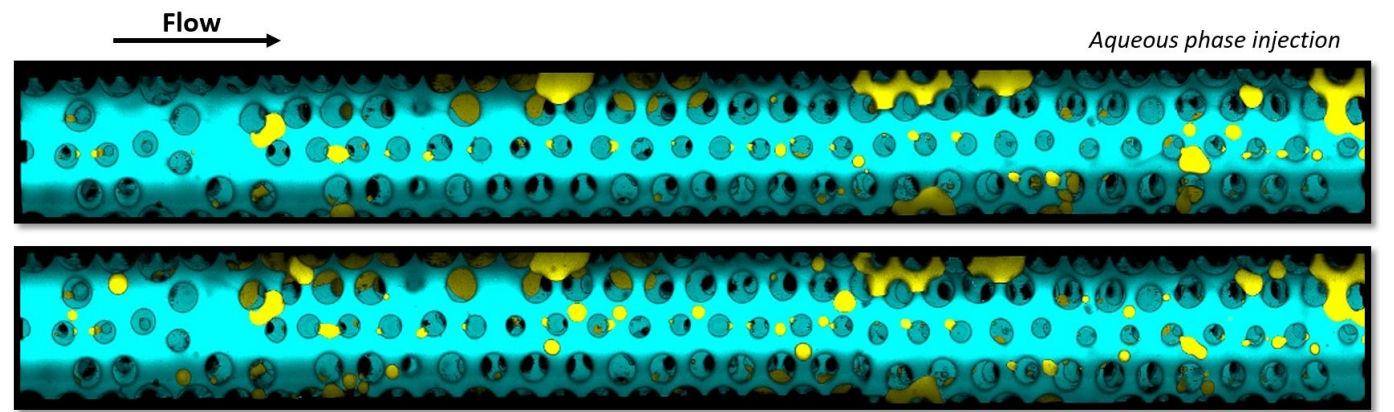

Microcapsules + Aqueous phase injection

Figure 4.17: 3D image of device "i", formed by the stack of 212 images, viewed from bellow.
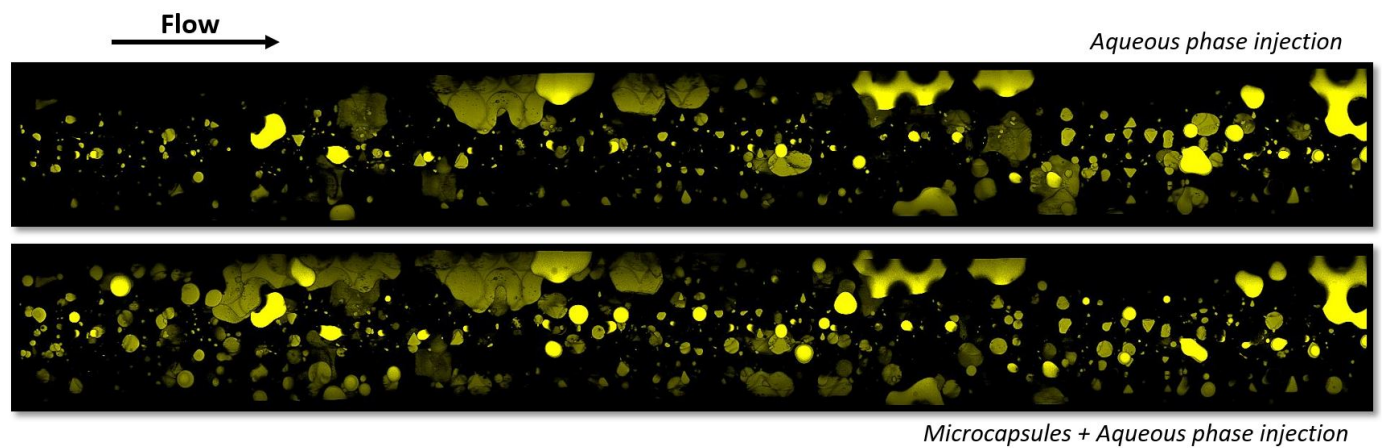

Figure 4.18: 3D image of device "i", formed by the stack of 212 images, viewed from bellow without the display of the aqueous phase.

Despite the fact that it was not possible to observe the microcapsules entering the device, in this experiment it was observed an increase of the oil phase after the capsules injection. The explanation to this phenomenon is that the oil phase that appeared in the region visualized was mobilized from the entrance of the porous medium.

\subsection{2}

\section{Results using devices "I" and "II"}

New devices were then produced and labeled in roman numerals after more knowledge in the experimental technique was developed during the preliminary experiments. In order to avoid repetitive observations and conclusions, from now on, only images that lead to important conclusions will be shown.

Device "I" had a porosity of $38.7 \%$ and a permeability of 232 D. Device "II", the porosity and the permeability were $42,0 \%$ and $215 \mathrm{D}$, respectively.

It is important to mention that all five experiments presented in this section were performed under the same experimental conditions, changing only the position for image acquisition (in the first experiment in the middle of the porous device, and then focusing on the inlet chamber and on the beginning 
of porous medium). Of course, their results are not exactly the same, since a complete reproducibility is impossible to be obtained due the nature of the porous media flow. However, each experiment highlights important details that help to build an experimental argument regarding the effects of microcapsules injection.

A very important result observed during most experiments, after the microcapsules injection, was the observation of oil leaving the porous device downstream. This oil mobilization is shown marked by the red circle in Fig. 4.19. This observation leads to the conclusion that part of the oil was mobilized by the microcapsules suspension after its injection and pushed out of the porous medium through the exit tube. It is important to remember that in all experiments, before injecting the microcapsules, the aqueous phase injection steady state was reached and, positively, no more oil would leave the device through the exit tube.

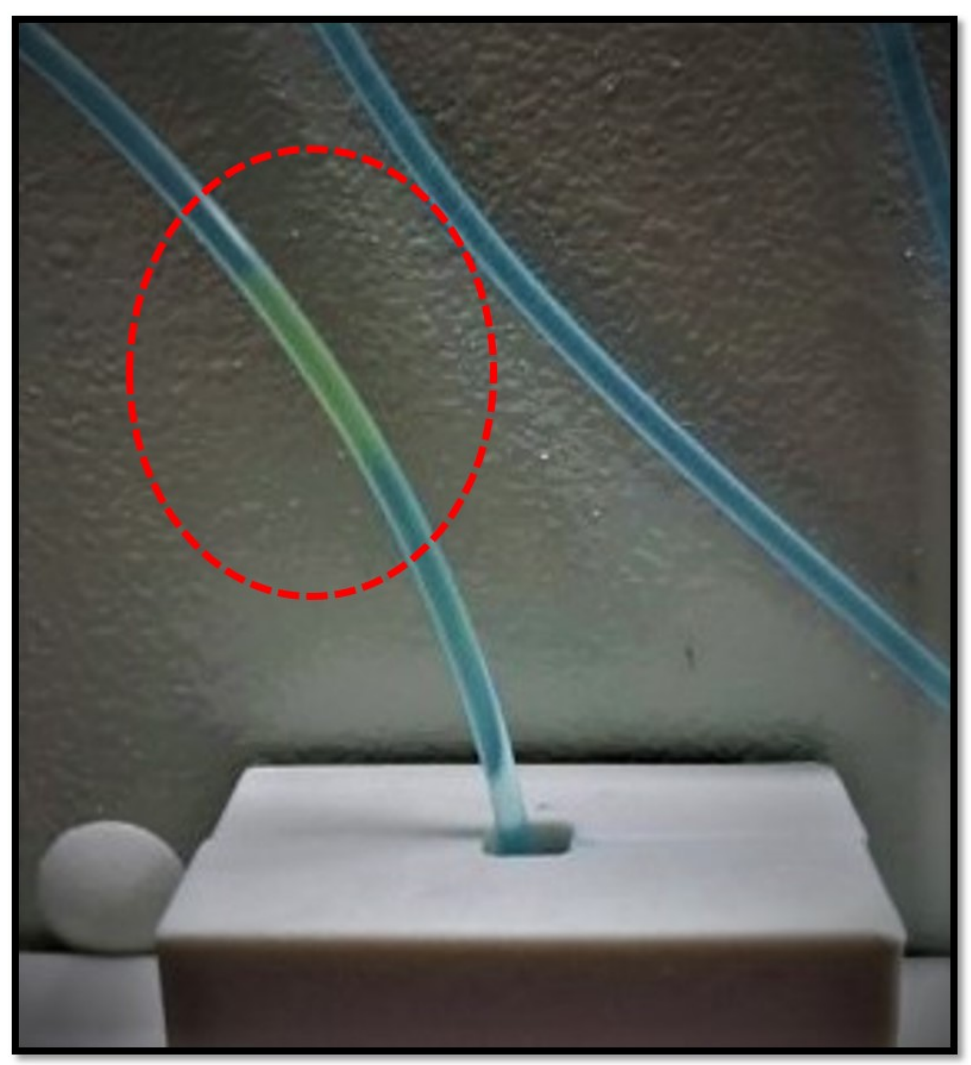

Figure 4.19: Oil leaving the porous device in the exit tube.

Clearly, it is possible to quantify, approximately, the amount of oil leaving the device using the separation of phases in the collection vial. However, the analysis of the oil recovery by that method was not performed as it differs from the scope of this work. 


\subsubsection{1}

\section{First experiment}

Using device "I" and following the experimental procedure chosen after the preliminary experiments and presented in the Procedure section, 3.2, the first experiment presented a similar result from the preliminary ones as showed in Fig. 4.20. The figure shows a view from the middle section of the device. The image acquisition for this experiment was done from one side only, without rotating the device.
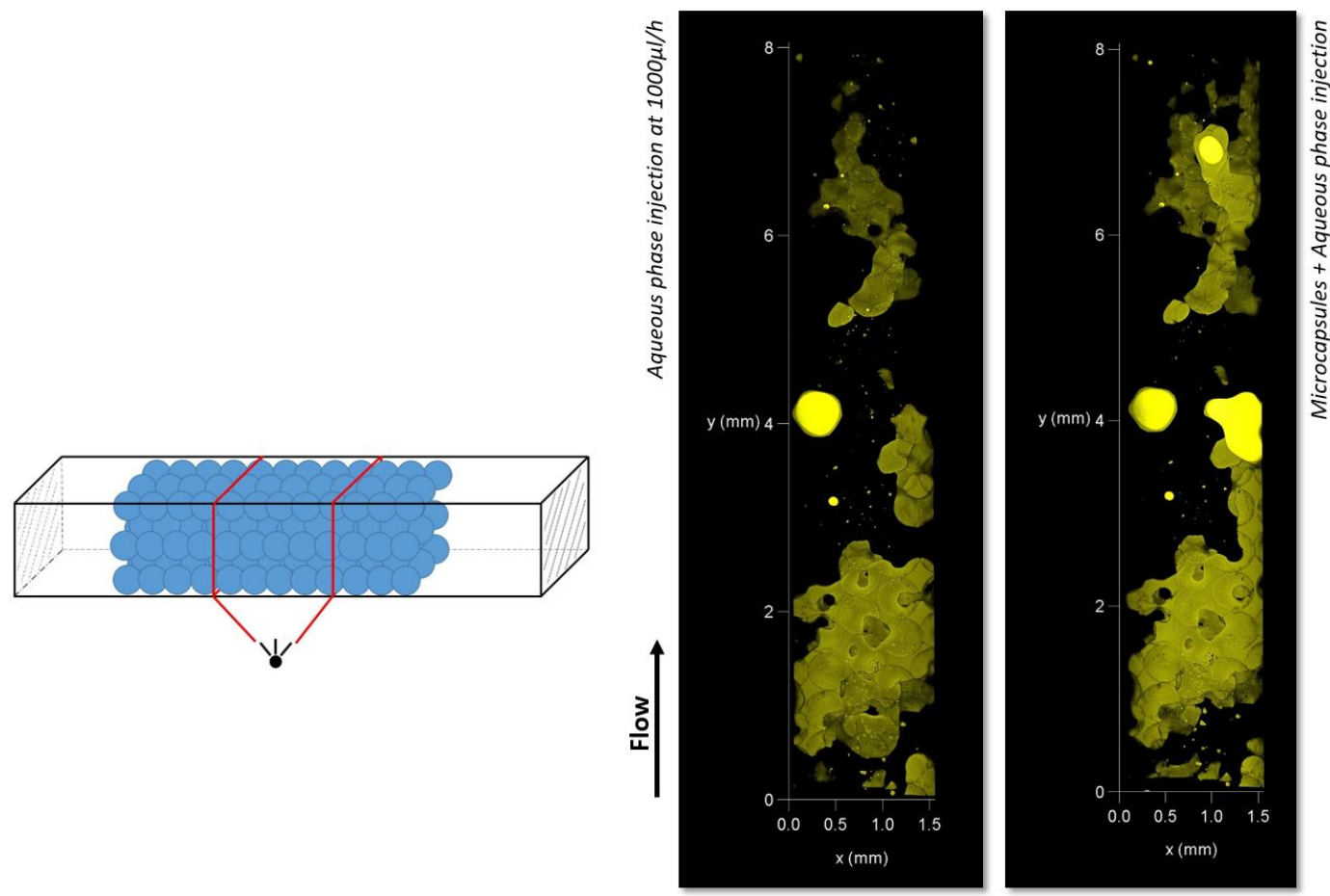

Figure 4.20: Bottom view from the middle section of the porous medium, as shown in the sketch. In the left is the image obtained after the injection of aqueous phase and in the right is the image obtained after the injection of aqueous phase with the microcapsules. The aqueous phase was hidden to facilitate the analysis.

In Figure 4.20, as in the preliminary experiment showed in Fig. 4.18, an increase of the oil phase after the capsules injection was observed, but the microcapsules did not appear in this region of porous medium. The explanation to this is that the additional oil that appeared in the visualization was mobilized from the entrance of the porous medium.

From this point on, the experiments were performed using the position adaptation at the device holder, and the visualized region at the beginning of the device. This was done aiming to better visualize the microcapsules and its effects on the flow. 


\subsubsection{2}

\section{Second experiment}

The second experiment was the one in which the microcapsules were clearly mapped for the first time inside the porous medium. It was performed using device "II".

In this experiment, and all others from now on, the region investigated includes part of the inlet chamber and the beginning of the porous medium, as shown in Fig. 4.21

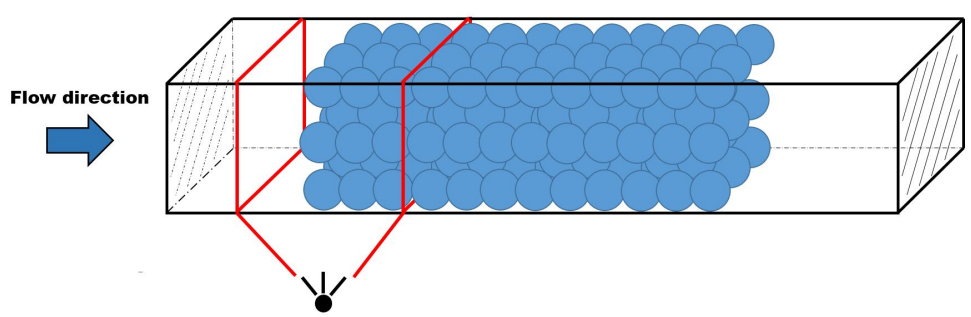

Figure 4.21: Sketch showing the region where image were acquired after the second experiment.

A clear distinction between the inlet chamber and the porous medium is quite obvious when the aqueous phase is also displayed in the image, as shown in Fig. 4.22. However, for better visualization of the oil and the microcapsules, most of the images from now on will numerically hide the aqueous phase. Whenever this is done, to make clear where the inlet chamber ends and the porous medium starts, a red line will be drawn marking the boundary of the porous medium.

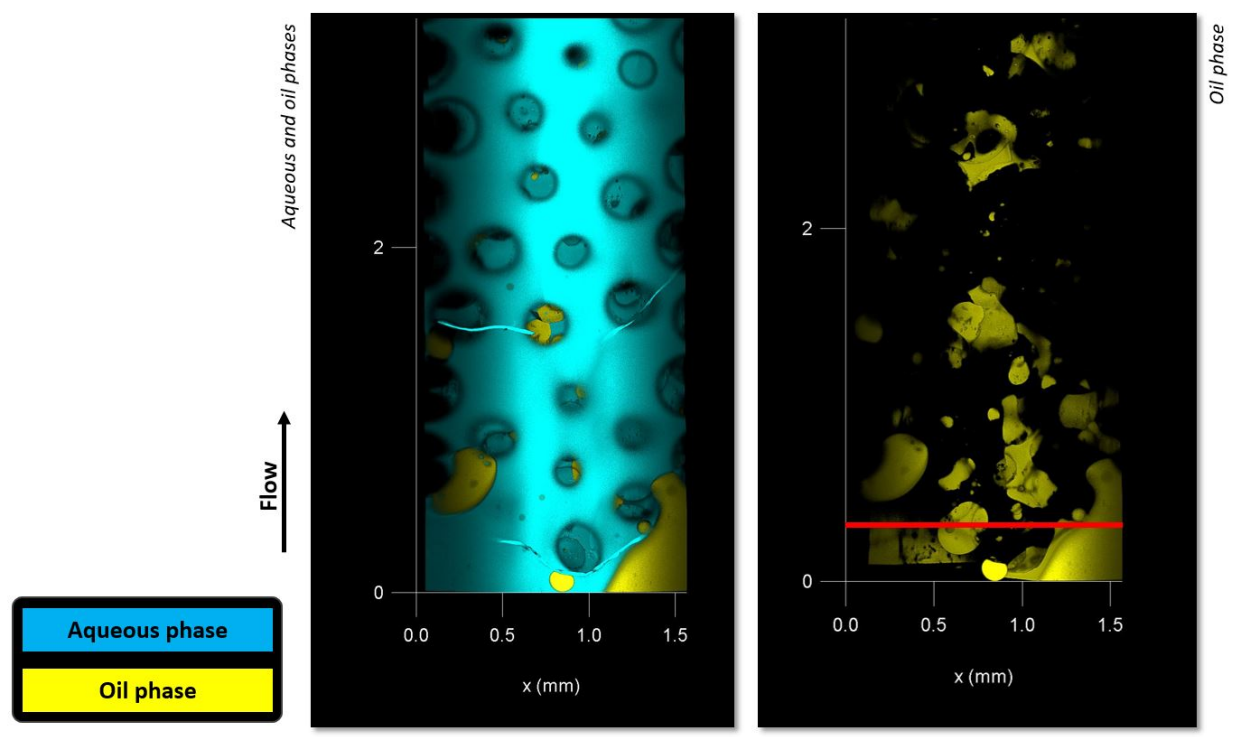

Figure 4.22: Images of the region investigated with and without showing the aqueous phase. To mark the boundary of the porous medium, a red line was drawn in the image hiding the aqueous phase. 
It was also the first experiment in which the visualization was performed from both sides of the porous device. This was obtained by axially rotating the device holder, as explained in Experimental Procedure chapter. So, from now on, whenever this technique was used, images obtained are labeled as from side "A" or from side "B". After each injection step an acquisition was performed from side "A" and then, the device holder was axially rotated to perform an acquisition from side "B".

Figure 4.23 shows the view from side "A" before and after the suspension of microcapsules was injected. Despite the microcapsules penetrated the entrance of the porous medium no much displacement was observed, probably because the microcapsules were concentrated in a region where no much oil was present.
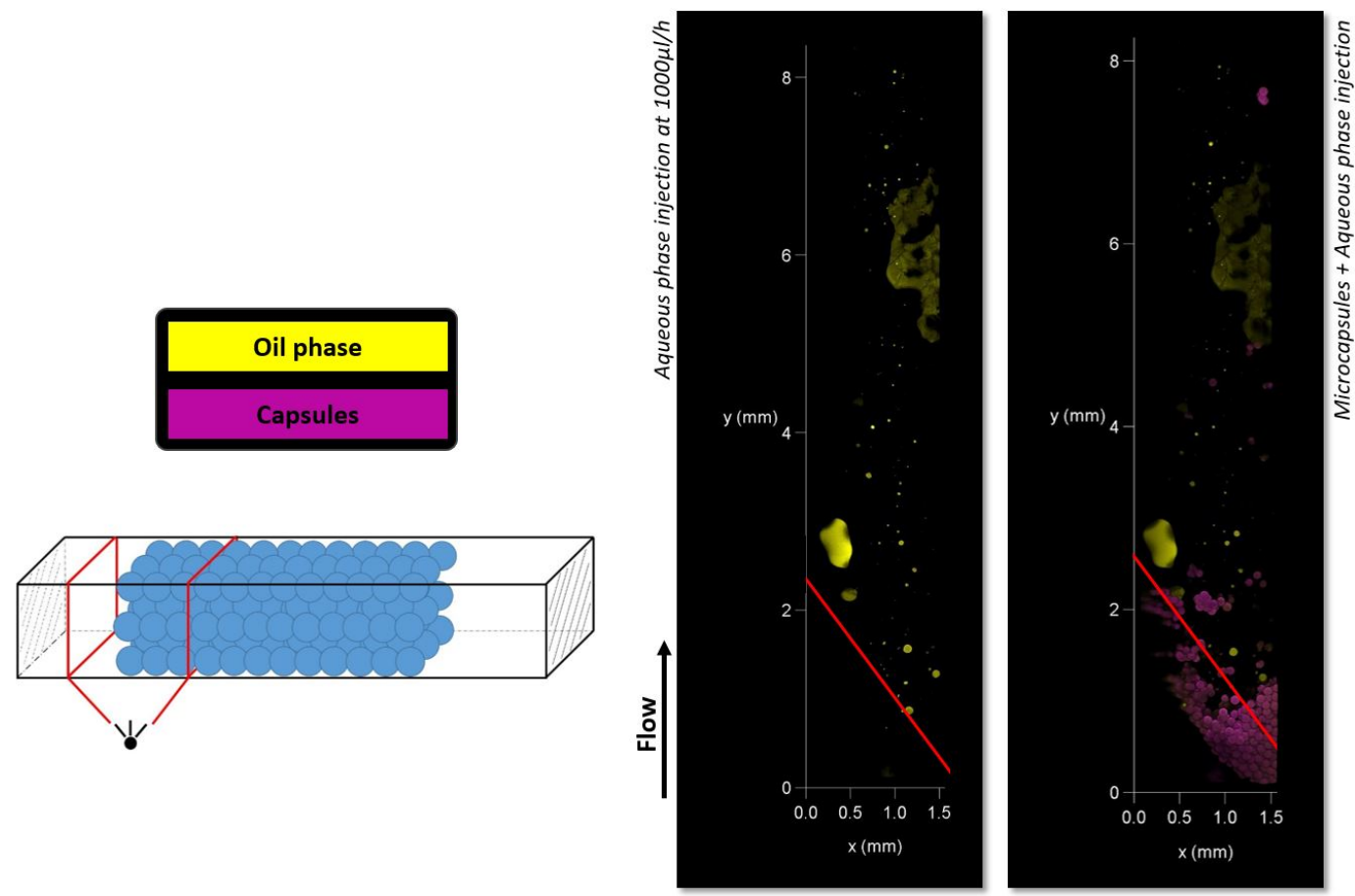

Figure 4.23: Bottom view from side "A" of device "II" in the second experiment. In the left is the image obtained after the injection of aqueous phase and in the right is the image obtained after the injection of aqueous phase with the microcapsules. The aqueous phase was hidden to facilitate the analysis. A red line was drawn to mark the boundary of the porous medium.

Figure 4.24 shows a perspective view from side "A" before and after the injection of microcapsules. 

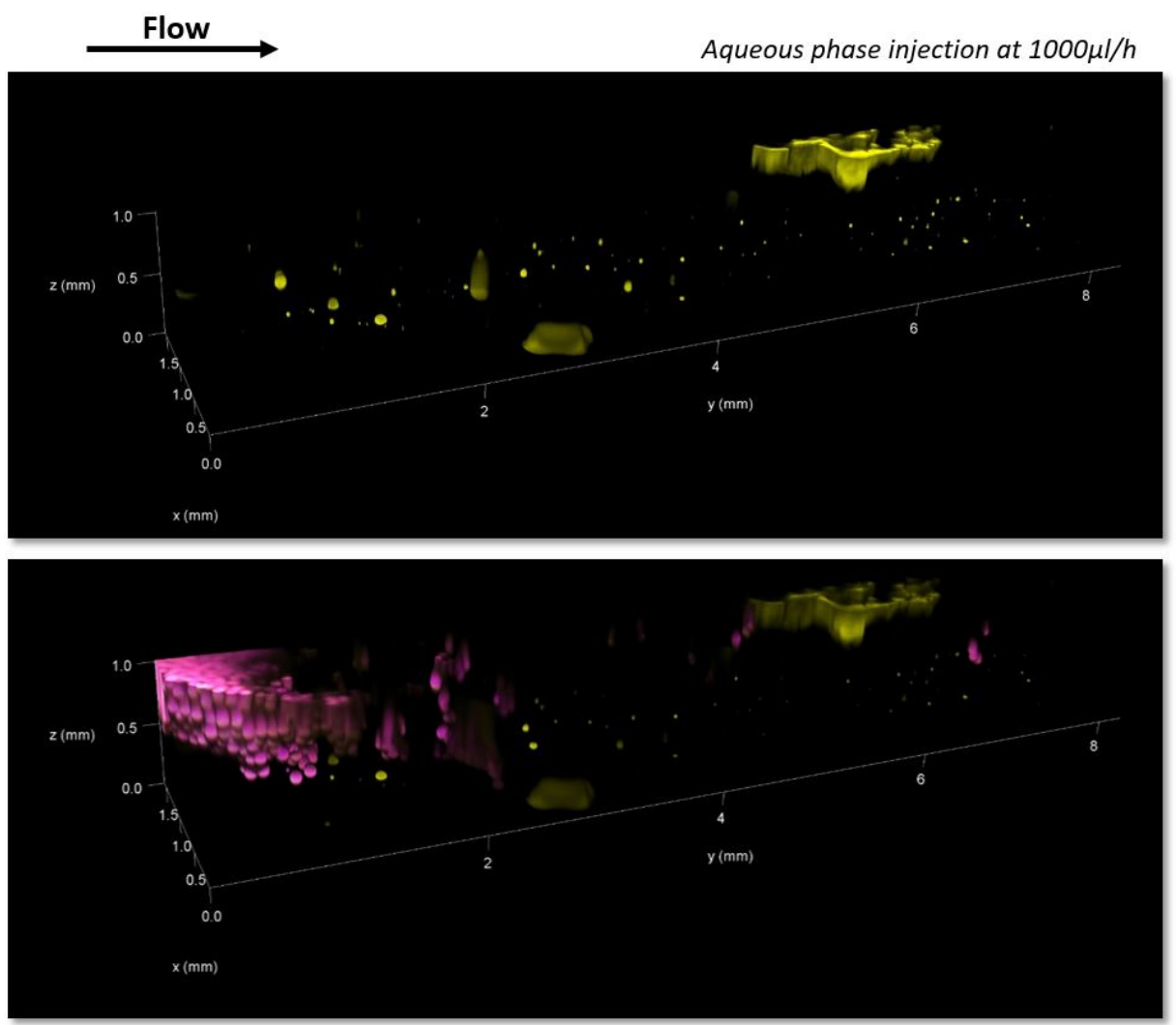

Microcapsules + Aqueous phase injection

Figure 4.24: Perspective view before and after the microcapsules injection in the second experiment. Visualization from side "A" with the aqueous phase hidden.

Figure 4.25 shows a detailed visualization of a region in Fig. 4.24 after the microcapsules injection. It can be seen that some microcapsules were able to penetrate further into the region near the ganglion. However, not many of them reached the region, and thus no effect was observed regarding mobilizing the ganglion.

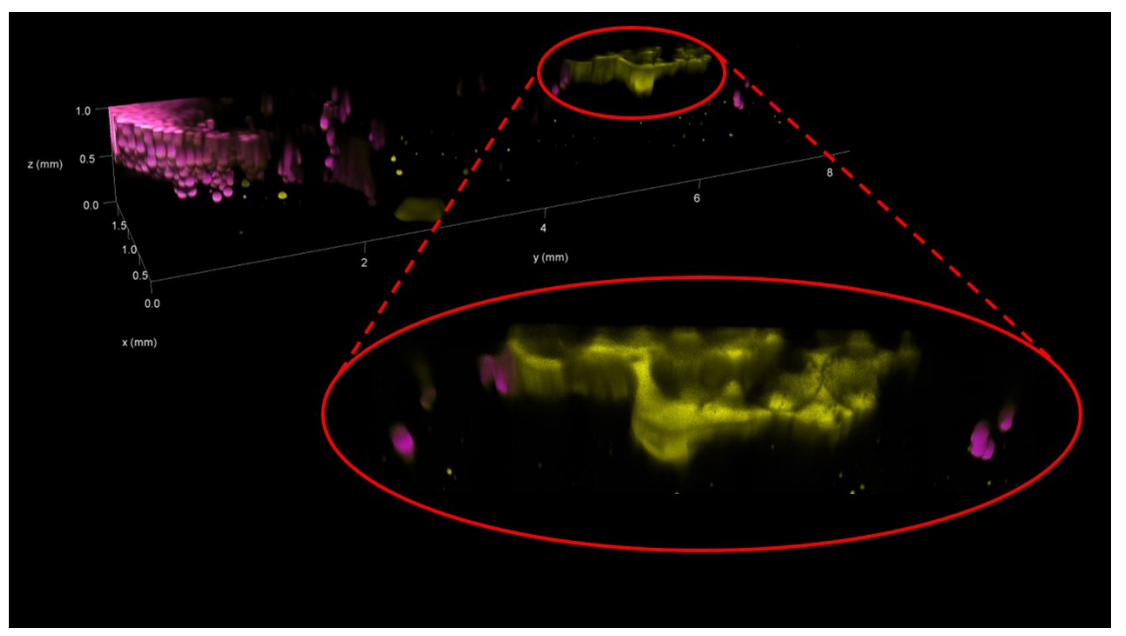

Figure 4.25: Detail of a oil ganglion that was not mobilized after the microcapsule blocking some pores near its position. 
The observation from the region surrounding the ganglion of Fig. 4.25 led to the conclusion that for mobilizing a ganglion, fully or partially, many more capsules were needed than those shown in the image.

The view from side "B", before and after the microcapsules injection, shown in Fig. 4.26 present a similar scenario to the observed from side "A".

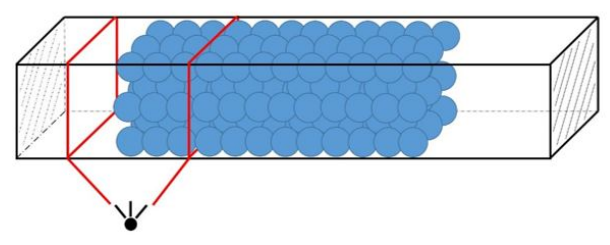

Oil phase

Capsules
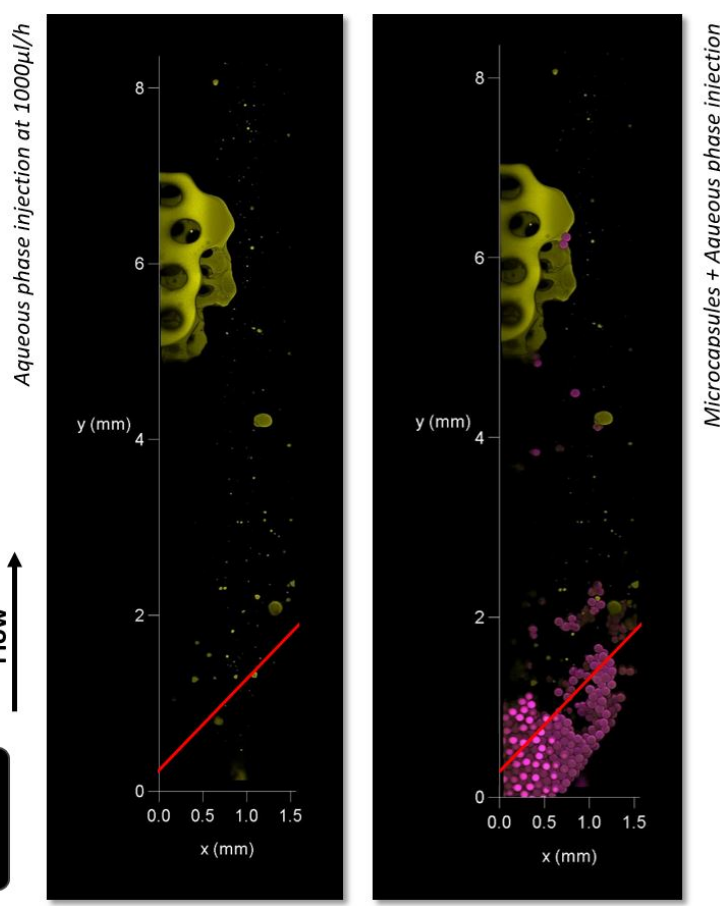

Figure 4.26: Bottom view from side "B" of device "II" in the second experiment. In the left is the image obtained after the injection of aqueous phase and in the right is the image obtained after the injection of aqueous phase with the microcapsules. The aqueous phase was hidden to facilitate the analysis. A red line was drawn to mark the boundary of the porous medium.

\subsubsection{3}

\section{Third experiment}

The third experiment was performed using device "I" again. In fact, from now on, all the experiments using homogeneous devices used device "I". In this experiment, more oil was left inside the entrance region than the second experiment. In this experiment only acquisitions from side "A" were performed.

Figure 4.27 shows the entrance region of the porous medium, before and after the microcapsules injection. It is even possible to observe that a large oil ganglion, circled by white, was initially concentrated at the inlet region, before the beginning of the porous medium. 

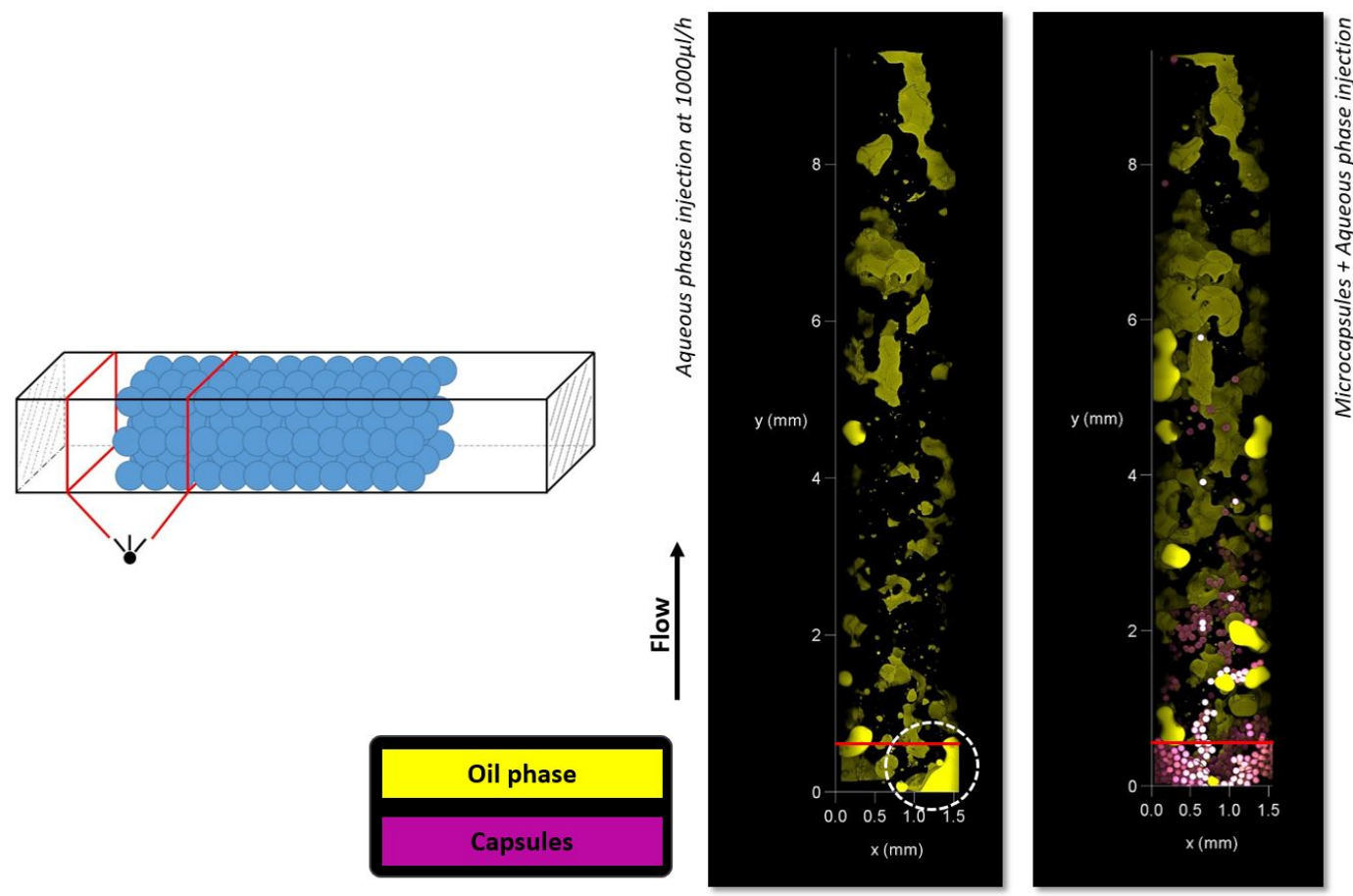

Figure 4.27: Bottom view from side "A" of device "I" in the third experiment. In the left is the image obtained after the injection of aqueous phase and in the right is the image obtained after the injection of aqueous phase with the microcapsules. The aqueous phase was hidden to facilitate the analysis. A red line was drawn to mark the boundary of the porous medium.

After the microcapsules were injected, it is possible to see that a good amount of oil was displaced. Analyzing Fig. 4.27, it is possible to see that a large number of microcapsules penetrated the device. Many oil ganglia were formed in different points of the porous medium after the injection. Some ganglia that kept their position changed their shapes, probably due the effects of the microcapsules blockage.

\subsubsection{4}

\section{Fourth experiment}

In the fourth experiment, also using device "I" and performing image acquisition from only side "A", led to results very similar to the third one.

Figure 4.28 shows a bottom view before and after the microcapsules injection. 

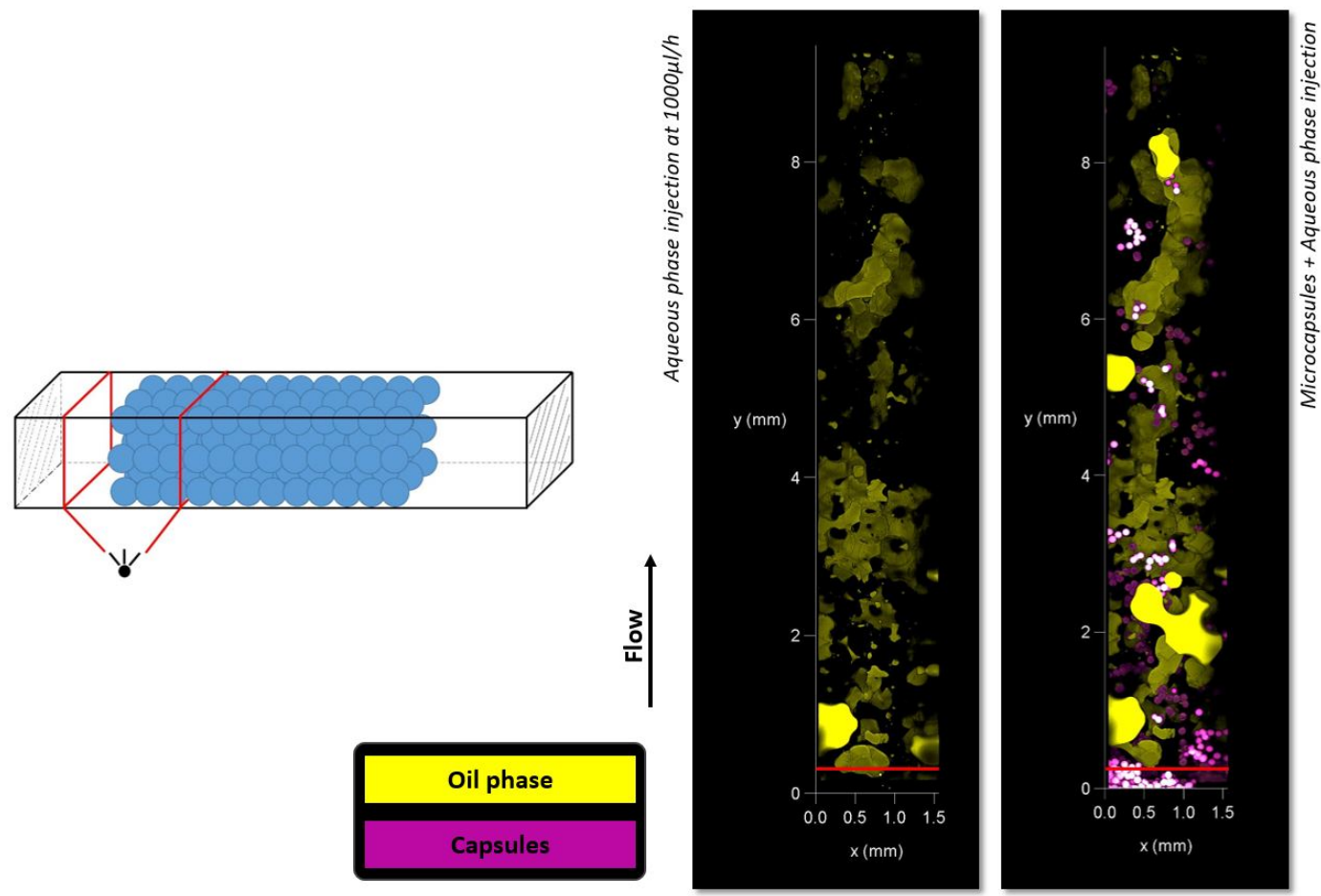

Figure 4.28: Bottom view from side "A" of device "I" in the fourth experiment. In the left is the image obtained after the injection of aqueous phase and in the right is the image obtained after the injection of aqueous phase with the microcapsules. The aqueous phase was hidden to facilitate the analysis. A red line was drawn to mark the boundary of the porous medium.

Figure 4.28 highlights another aspect observed. It appears again that more oil was present after the microcapsules injection than before. This could be explained by the displacement of large accumulation of oil at the inlet region of the device, not visualized in this experiment. In order to investigate this, a fifth experiment was performed, moving the visualization window further into the entrance of the device.

\subsubsection{5}

\section{Fifth experiment}

The fifth experiment, also with device "I" presents results with visualization from both sides of the device. It also explores the behavior of the flow in the inlet region prior to the porous medium.

Figure 4.29 shows the bottom view from side "A" before and after the microcapsules injection. Two large ganglia can be seen concentrated at the inlet before the porous medium region, one in the left side and one in the right side of the device. These ganglia were clearly moved by the microcapsules that concentrated between them. From the side "A" bottom view, it seems that the microcapsules did not penetrated much into the porous medium, but view 
from different perspectives will show that more capsules were able to penetrate much further. In any case, the image after the injection of microcapsules shows that a lot of oil was mobilized downstream the entrance region.
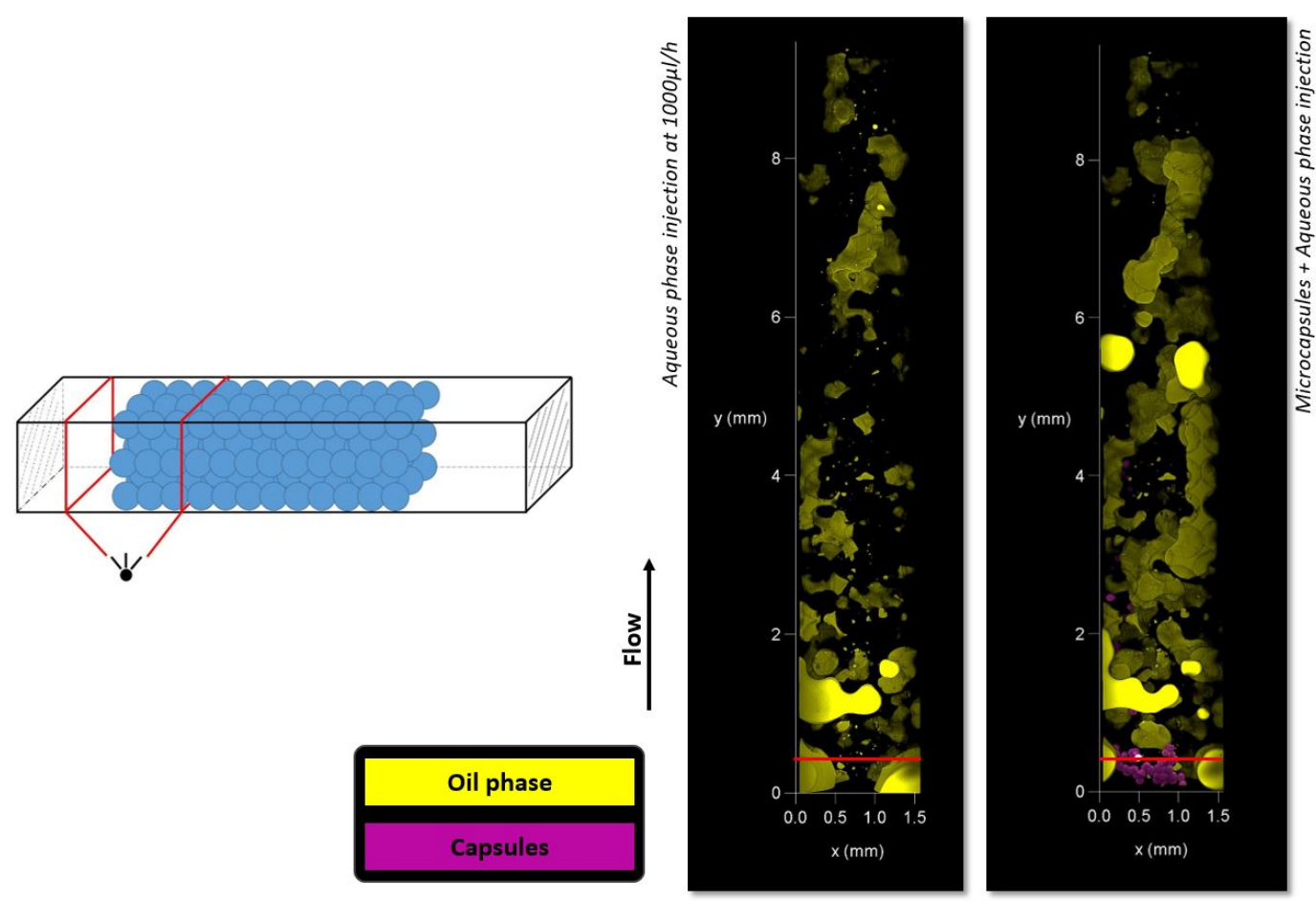

Figure 4.29: Bottom view from side "A" of device "I" in the fifth experiment. In the left is the image obtained after the injection of aqueous phase and in the right is the image obtained after the injection of aqueous phase with the microcapsules. The aqueous phase was hidden to facilitate the analysis. A red line was drawn to mark the boundary of the porous medium.

The observations made about Fig. 4.29 are reinforced when analyzing the 3D perspective, Fig. 4.30, built by stacking 228 2D slices captured from side "A" before and after the microcapsules injection. The two ganglia initially positioned at the entrance are much clearer viewed, as well as the effect of their partial mobilization further down the porous medium. The image highlights also the fact mentioned before that microcapsules were able to penetrate in the porous medium further downstream. 


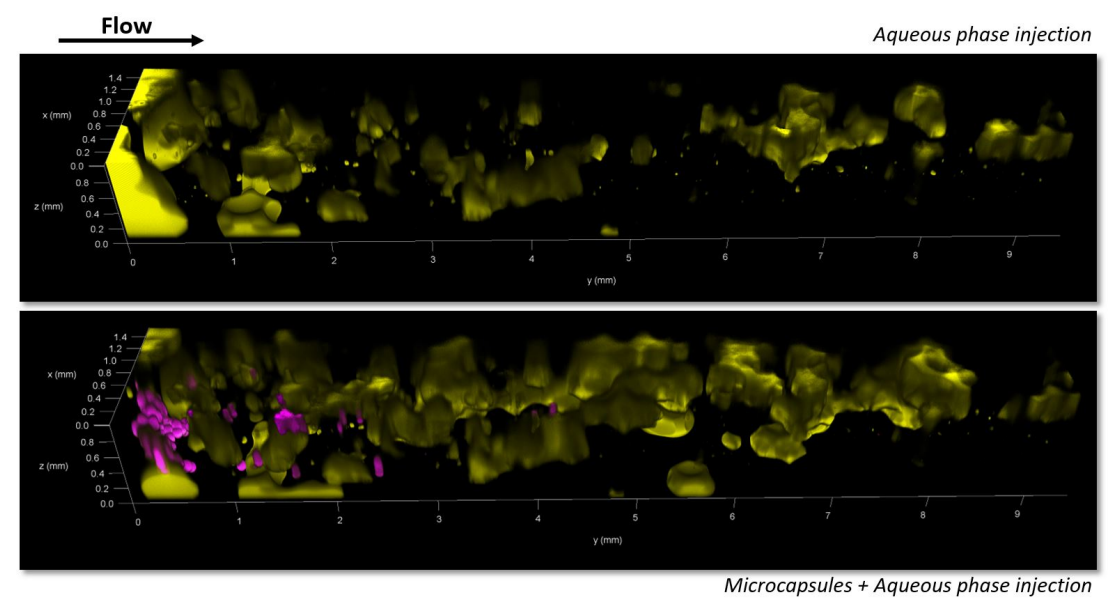

Figure 4.30: 3D perspective view built by stacking 228 2D slices captured from side "A" before and after the microcapsules injection. The aqueous phase was hidden to facilitate the analysis. Notice the microcapsules located further downstream in the porous medium.

Figures 4.31 to 4.35 display images obtained visualized from side "B". In Figure 4.31, it can be seen that only one of the large ganglia viewed from side "A" reach the other side of the device. After the microcapsules injection, in the same figure, it can be noticed that microcapsules penetrated much farther into the porous medium than in the other side. The mobilization of oil ganglia can be observed very clearly in the regions where large amounts of microcapsules were concentrated and blocked the flow. 

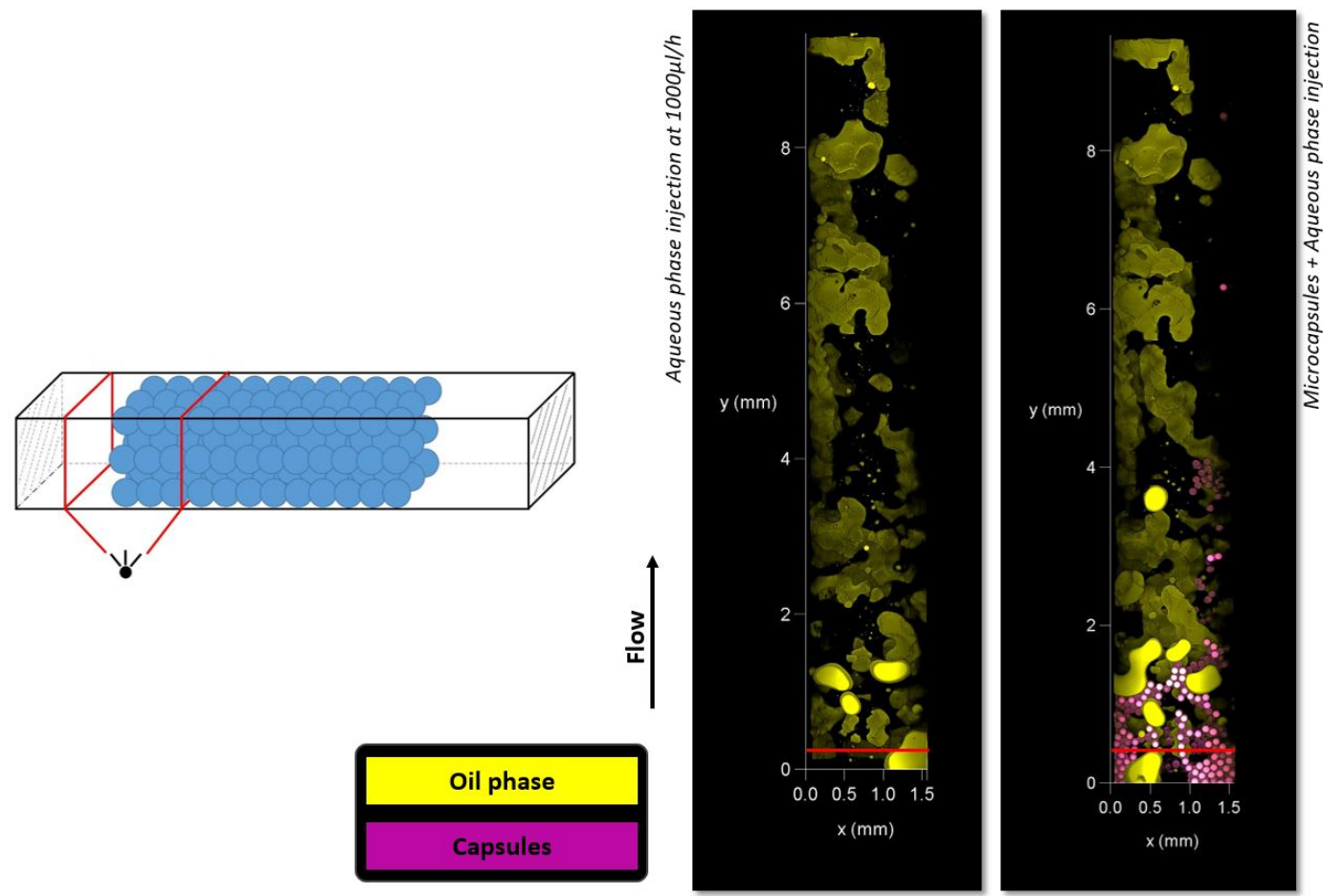

Figure 4.31: Bottom view from side "B" of device "I" in the fifth experiment. In the left is the image obtained after the injection of aqueous phase and in the right is the image obtained after the injection of aqueous phase with the microcapsules. The aqueous phase was hidden to facilitate the analysis. A red line was drawn to mark the boundary of the porous medium.

Figure 4.32 shows a 3D perspective built by stacking $2282 \mathrm{D}$ slices captured from side "B" before and after the microcapsules injection. 


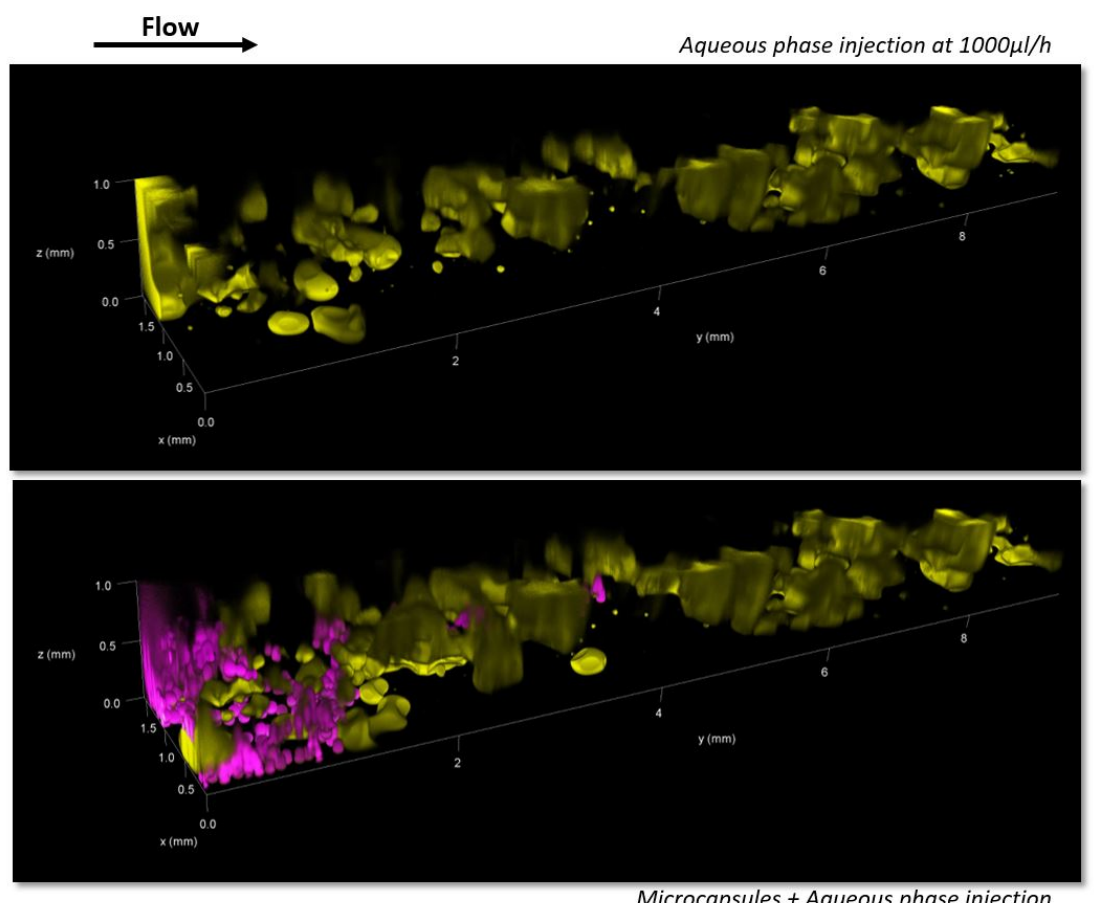

Figure 4.32: 3D perspective view built by stacking 228 2D slices captured from side "B" before and after the microcapsules injection. The aqueous phase was hidden to facilitate the analysis. Notice the presence of only one large ganglion at the inlet of the device before the porous medium.

It was possible to manipulate the $3 \mathrm{D}$ perspectives view from the images acquired from sides "A" and "B" to visualize how the porous medium could be fully reconstructed. This image manipulation is shown in Fig. 4.33. The three phases are shown, blue for the aqueous phase, yellow for the oil phase and purple for the capsules. The side " $\mathrm{A}$ " is the $3 \mathrm{D}$ reconstruction seen far from the point of view and the side " $\mathrm{B}$ " is the one that looks closer and with the clear visualization of the microcapsules. The flow direction was from the top part to the bottom part of the image, following the $y$ axis. 


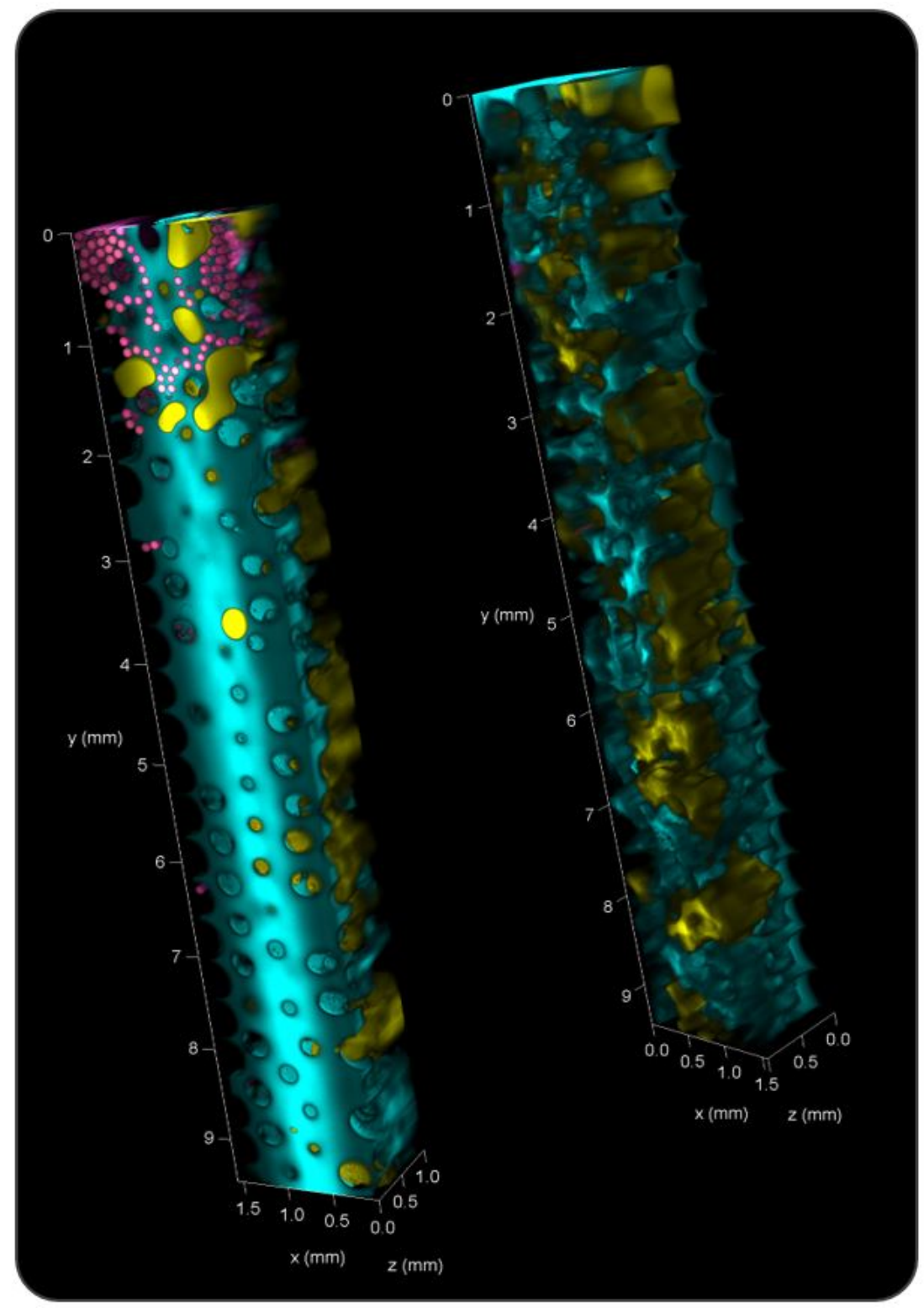

Figure 4.33: Observation of the full depth of the porous medium by combining the $3 \mathrm{D}$ images acquired from opposite sides.

Figure 4.34 shows a 2D slice obtained from side "B", at the stack position 17, before and after the microcapsules injection. In these images the aqueous phase was not hidden. It is possible to see the mobilization of the oil from the inlet to positions further downstream. 

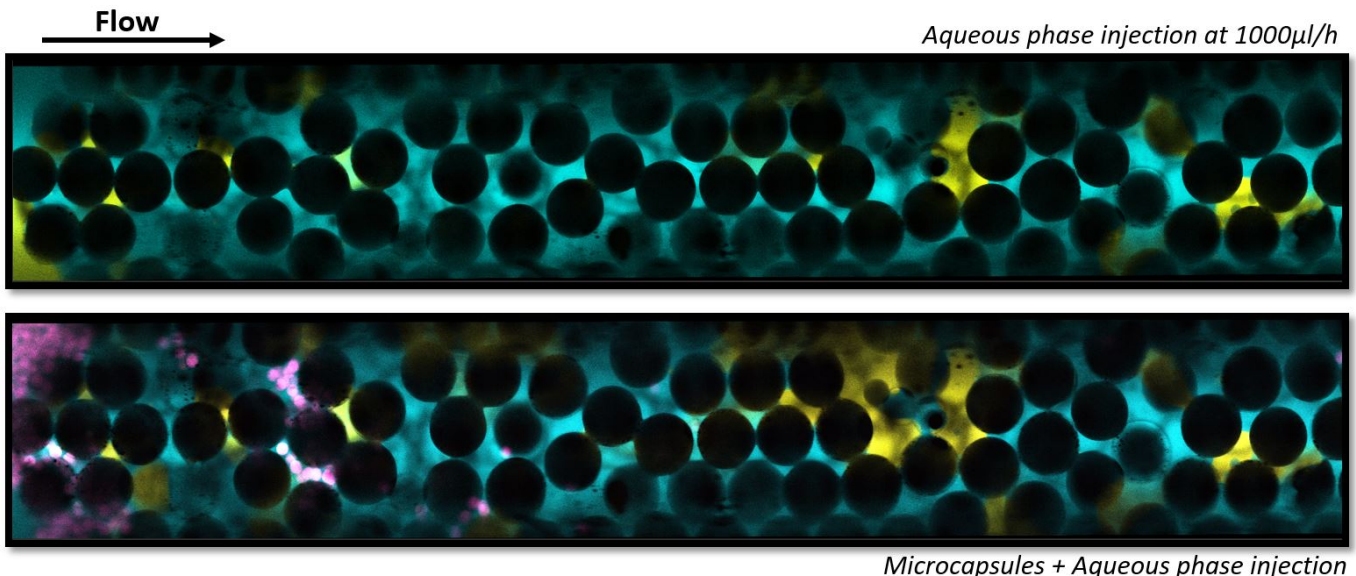

Figure 4.34: View from side "B" of a single 2D slice from device "I", at stack position 17 , before and after the microcapsules injection for comparison.

Figure 4.35 shows a zoom of a $2 \mathrm{D}$ slice obtained visualizing from side " $\mathrm{B}$ " made at the very beginning of the porous medium at the stack position 178. In this stack position, deeper into the device, it is much harder to get a complete clear image as those closer to the walls. Thus, besides the glass beads that are in the stack plane, black spots are observed. They are shades from spheres located in nearby planes. In any case, they do not compromise any comparison between before and after images, since they are still. 


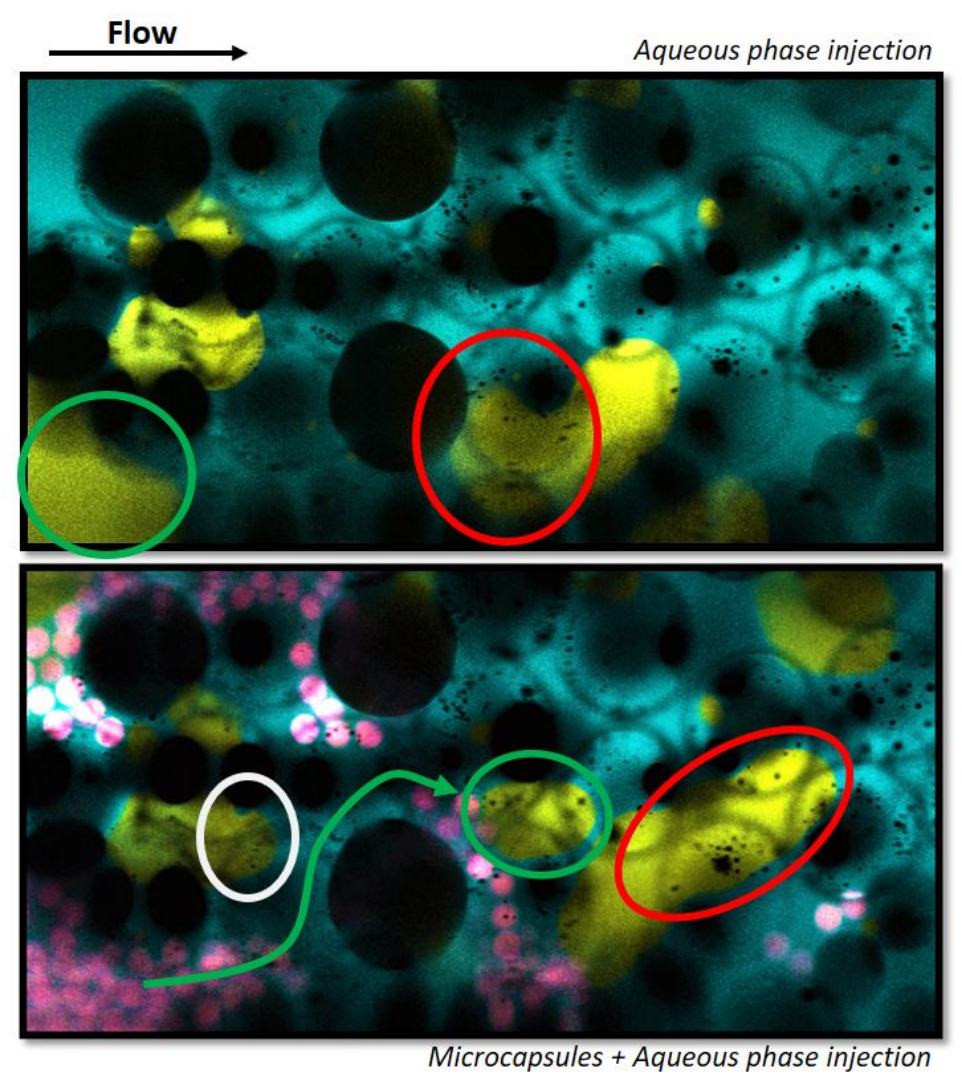

Figure 4.35: Detailed view from a single plane at the entrance of the device "I". The upper image is the plane before the microcapsules injection and the bottom image is the result after the microcapsules injection.

In the images of Fig. 4.35, it gets very clear the microcapsules penetrating in the porous channels and how oil ganglia were mobilized by them. An interpretation of how these mobilizations occurred is suggested by the circles and arrows in the figure. The green marks show the probable movement that occurred with the oil ganglion at the entrance. The white mark shows a small shape deformation of the selected ganglion that was not fully mobilized. The red circles highlights the displacement of one oil ganglion downstream the porous medium.

\section{2}

\section{Visual qualitative results with stratified porous media}

Two stratified devices were used, devices "V" and "VI". The device "V" had $44.3 \%$ porosity and $182 \mathrm{D}$ of permeability. The device "VI" had $39.3 \%$ porosity and $180 \mathrm{D}$ of permeability. It is important to notice that, even having similar permeabilities, device " $\mathrm{V}$ " has more layers of large glass spheres than the device "VI". Also, it is convenient to remind that, as mentioned before, the coarse spheres are $425 \mu \mathrm{m}$ diameter and the fine spheres are $250 \mu \mathrm{m}$. The microcapsules used in the experiments had $104 \mu \mathrm{m}$ external diameter. 
Two experiments were performed, one with each device, here named sixth and seventh experiments. For the sixth experiment, the image was acquired from the wall that allows to see both large and small glass spheres. For the seventh experiment the device was visualized from the wall near to the large glass spheres (side "A") and the wall near to the small glass spheres (side "B").

\subsection{1}

\section{Sixth experiment}

In the sixth experiment, using device "VI", the aim was to investigate the microcapsules injection phenomenon from a transverse plane so that both coarse and fine spheres were visible.

Figure 4.36 shows the image acquired according to the sketch where is possible to see the coarse and fine spheres. The image was acquired without the fluorescence detector, so it is in shades of gray. No microcapsules injection was applied prior to this acquisition. The black spots viewed in the image are small defects on the large spheres or shades from other layers.

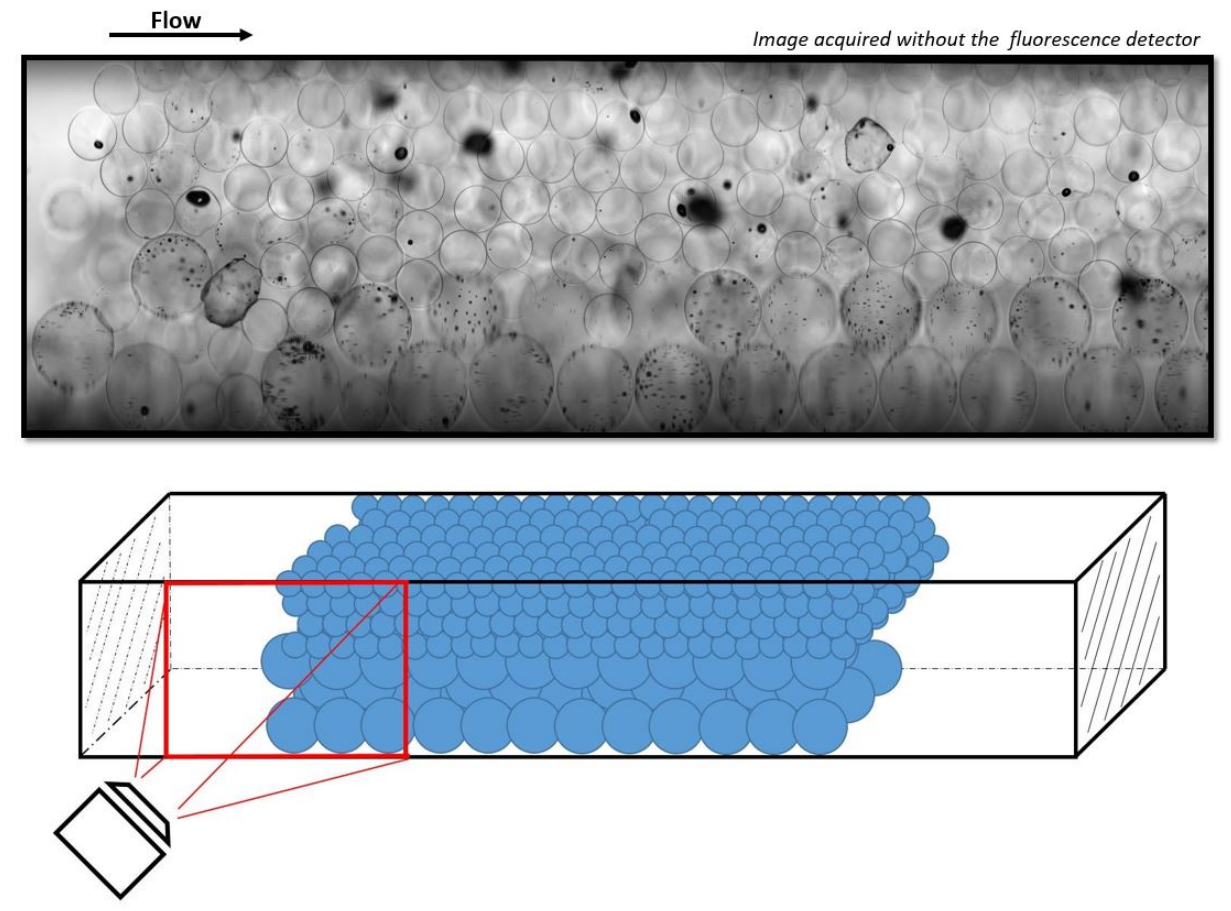

Figure 4.36: Image acquired without the fluorescence detector showing the plane of acquisition, according to the sketch, in which both coarse and fine glass spheres are observed.

The result before and after the injection of microcapsules is shown in Fig. 4.37. Again the aqueous phase was hidden and the coarse spheres are on the left side of the images and the fine spheres on the right side. The microcapsules are concentrated at the entrance of the fine spheres region, where they could not 
penetrate. On the other hand, no microcapsules were found inside the coarse spheres region investigated. This could mean that either they were not able to penetrate there or, more probably, that they were forced to pass through the coarse region.
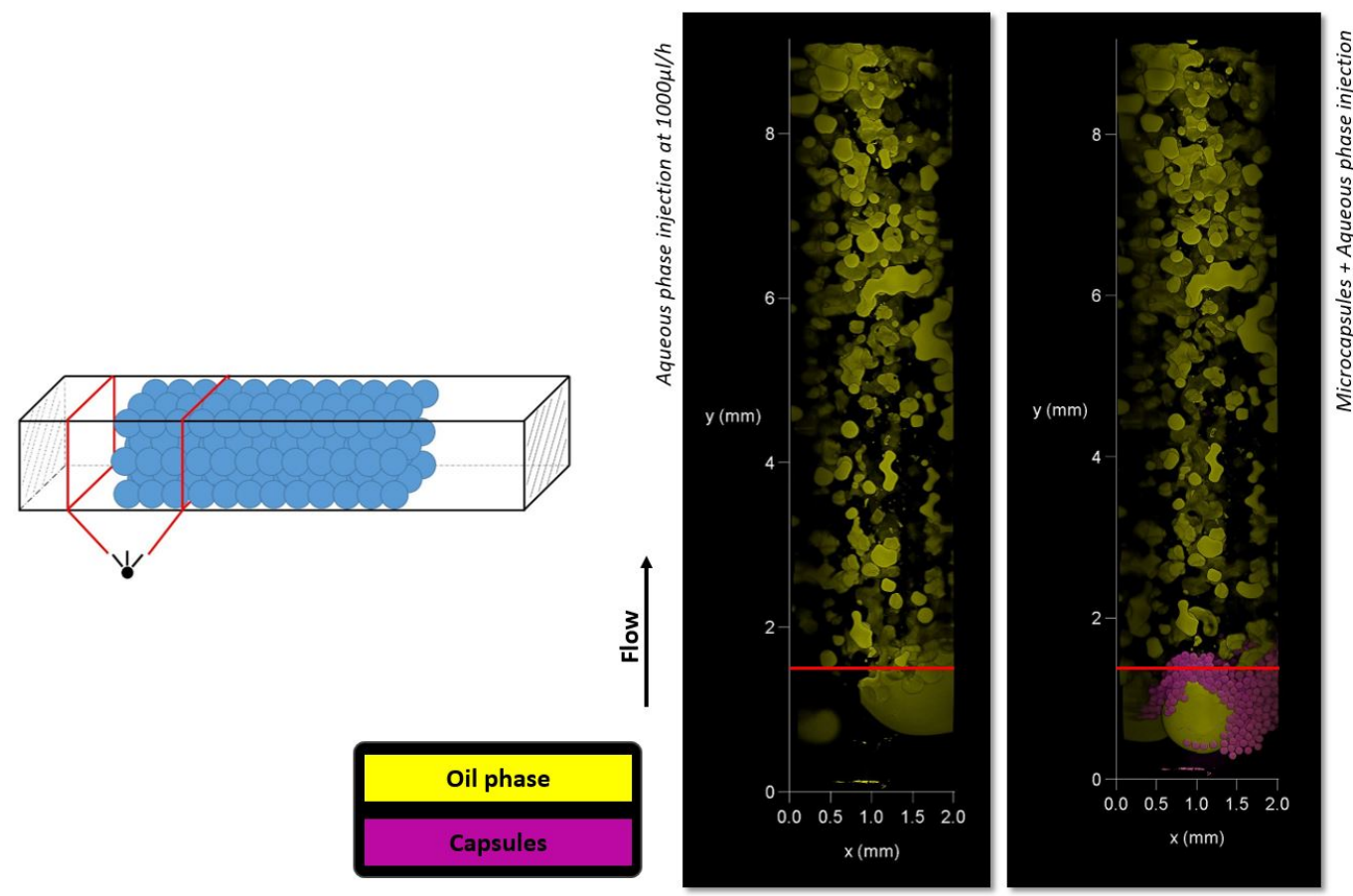

Figure 4.37: View from side "A" of the transversal plane of the device "VI" in the sixth experiment, before and after microcapsules injection. On the left of the image are the coarse spheres region and on the right are the fine spheres. The aqueous phase was hidden to facilitate the analysis. A red line was drawn to mark the boundary of the porous medium.

Perhaps, looking at the other side of the device, side "B", the doubt regarding the entrance or not of the microcapsules in the coarse region could enlightened.

In Fig. 4.38 a view from side "B" of the transversal plane of the device "VI" in the sixth experiment, before and after microcapsules injection is shown. Now, the coarse spheres are on the right side of the images and the fine spheres on the left side. 

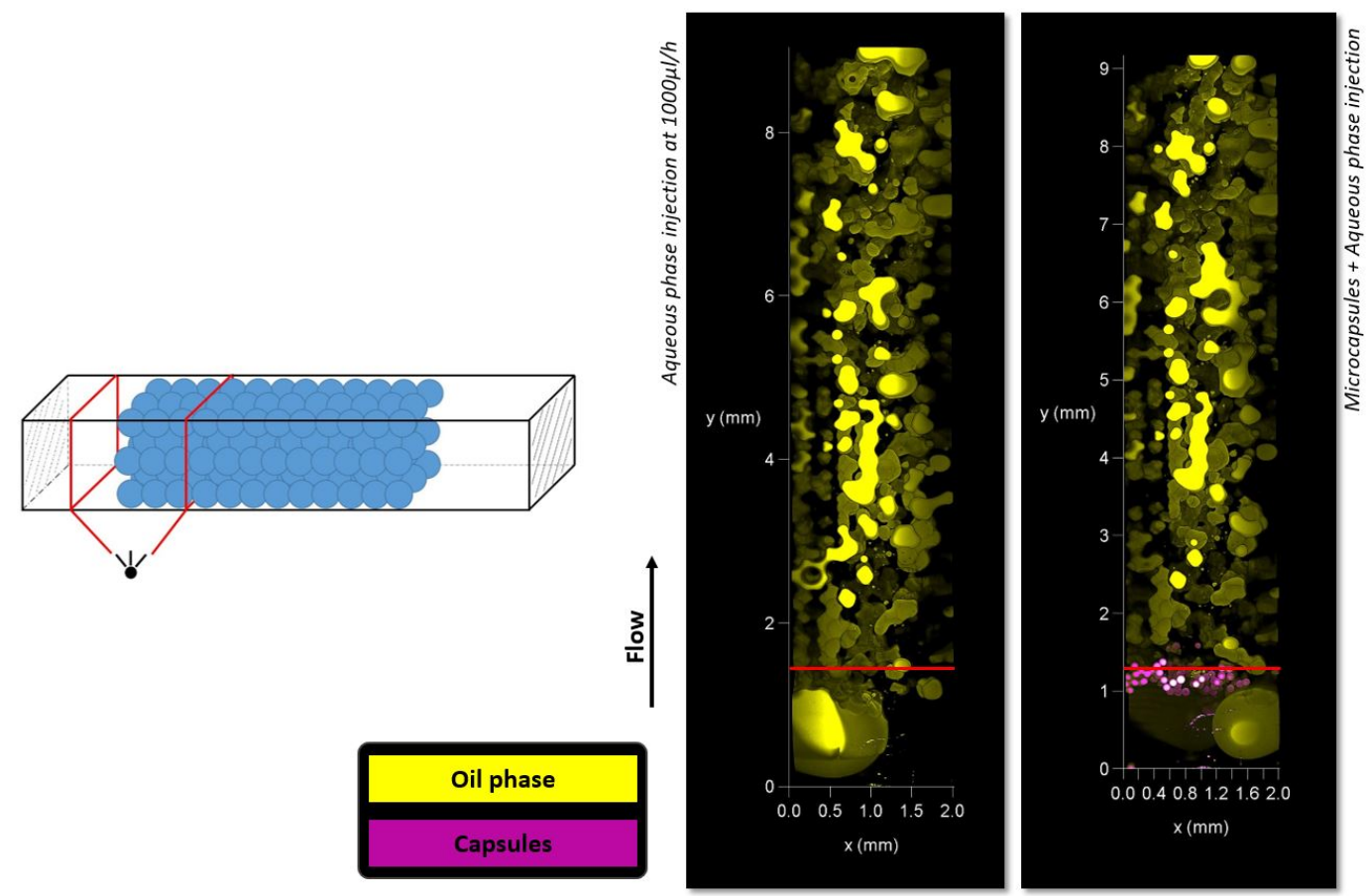

Figure 4.38: View from side "B" of the transversal plane of the device "VI" in the sixth experiment, before and after microcapsules injection. On the right of the image are the coarse spheres region and on the left are the fine spheres. The aqueous phase was hidden to facilitate the analysis. A red line was drawn to mark the boundary of the porous medium.

The images obtained also showed that the microcapsules concentrated at the entrance of the fine spheres region, where again they could not penetrate. Also no microcapsules were located inside the coarse spheres region investigated. However, the visualization from side "B" shows more clearly than the one from side "A" that oil ganglia were mobilized along both the coarse spheres region and, more discretely, the fine spheres region.

Also, images from both side "A" and "B" show an almost complete blockage of the entrance region by microcapsules. So, probably, higher fluid velocity was reached at the coarse spheres region. This fact, along with the fact that some oil ganglia mobilization was observed, may point that the second hypothesis mentioned before is correct, meaning that the microcapsules which penetrated the coarse spheres region were swept away from the visualization domain and promoted some oil ganglia mobilization.

Comparing the results from the sixth and the seventh experiment, a similarity on the fact that the microcapsules were blocking the entrance for the fine spheres region. However, contrarily to the sixth experiment, it is possibly to see many microcapsules located inside the porous medium, mostly near the interface between the coarse and fine spheres regions. This maybe be explained by the fact that the device used in the seventh experiment had more layers of 
coarse spheres than the one used in the sixth.

\subsection{2}

\section{Seventh experiment}

In this experiment the device "V" was used. The result before and after the microcapsules injection from the side of the large spheres is shown in Fig. 4.39 and from the side of the small spheres is shown in Fig. 4.41. Figure 4.40 shows a perspective view from the side of the coarse spheres.

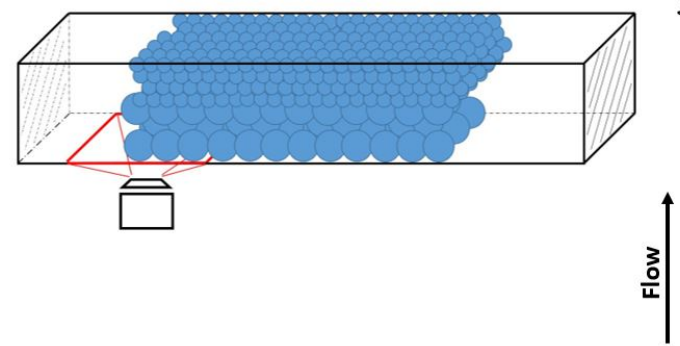

Oil phase

Capsules
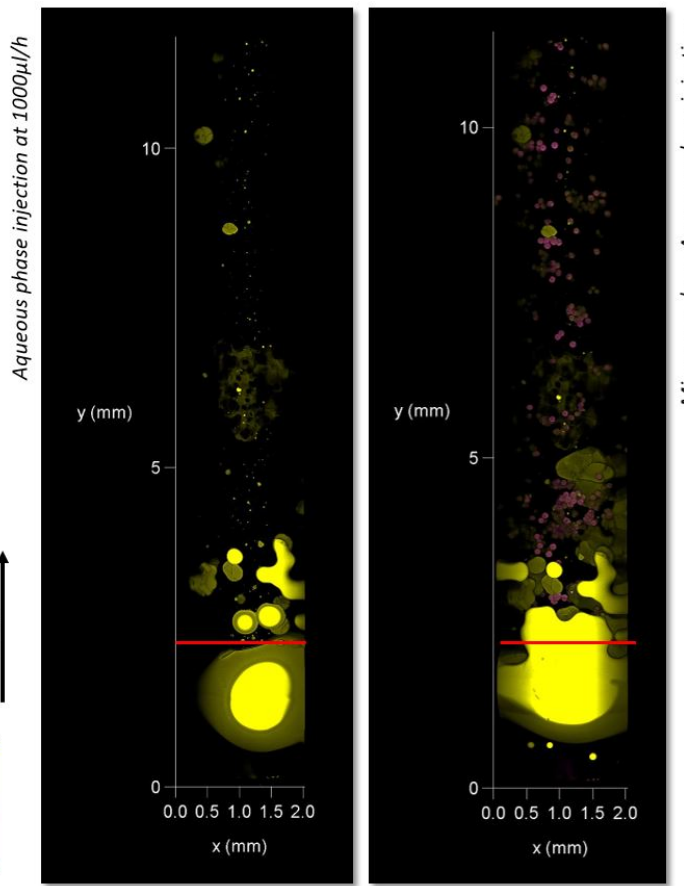

Figure 4.39: View from side of the coarse spheres of the device "V" in the seventh experiment, as shown in the sketch. In the left is the image obtained after the injection of aqueous phase and in the right is the image obtained after the injection of aqueous phase with the microcapsules. The aqueous phase was hidden to facilitate the analysis. A red line was drawn to mark the boundary of the porous medium. 

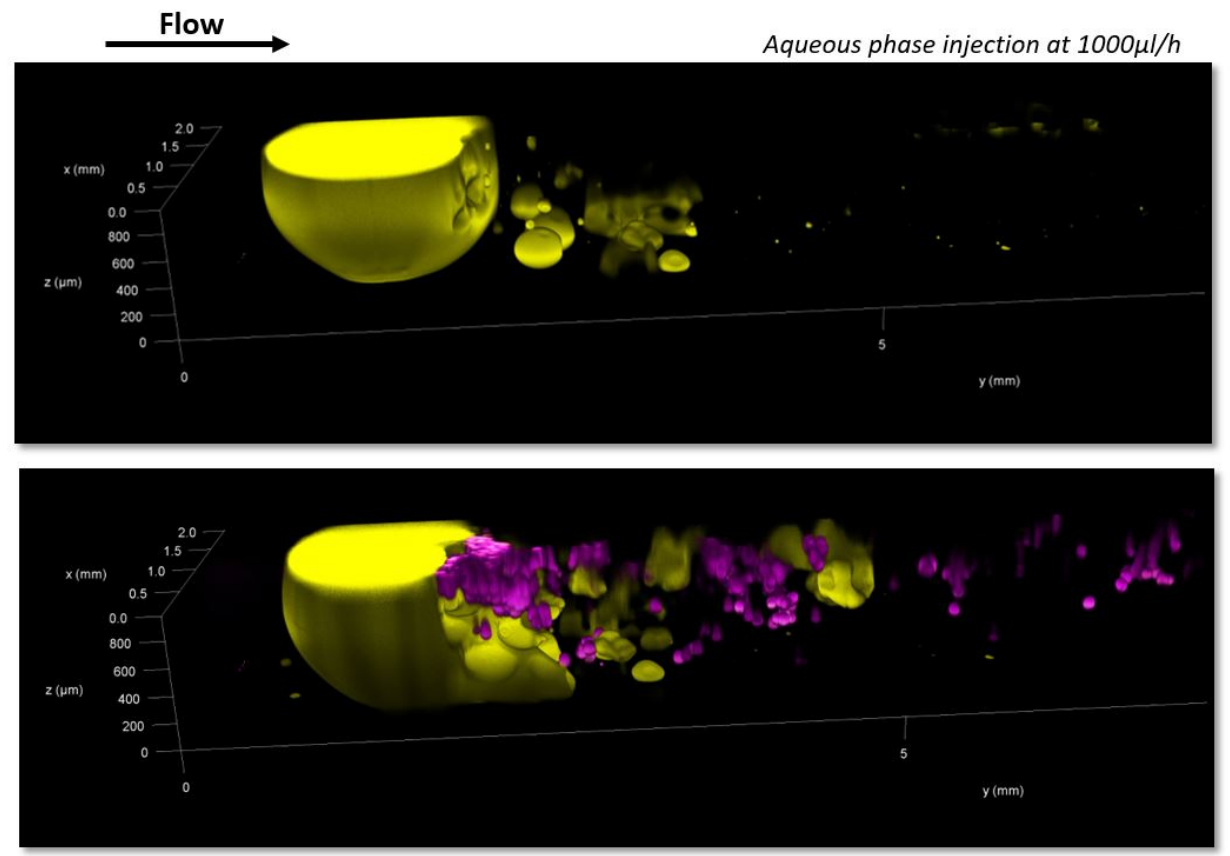

Microcapsules + Aqueous phase injection

Figure 4.40: 3D perspective view built by stacking 228 2D slices captured from the side of the coarse spheres before and after the microcapsules injection. The aqueous phase was hidden to facilitate the analysis.

From the side of the coarse spheres, it seems that the large ganglion positioned at the inlet of the device before the porous medium increased in volume, but when looking at the perspective view it is possible to conclude that the ganglion in fact was mobilized and part of it was forced downstream. 

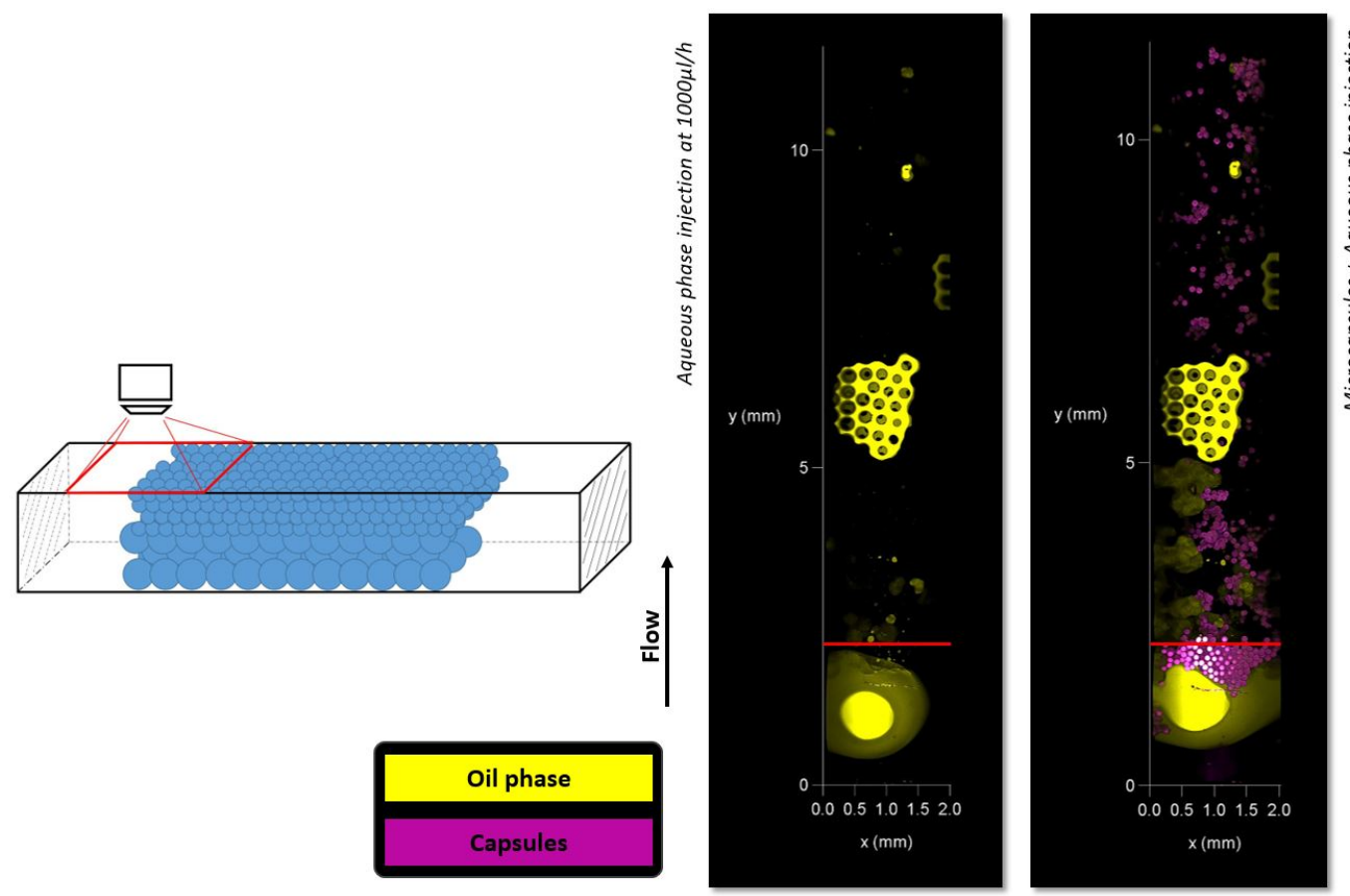

Figure 4.41: View from side of the fine spheres of the device " $\mathrm{V}$ " in the seventh experiment, as shown in the sketch. In the left is the image obtained after the injection of aqueous phase and in the right is the image obtained after the injection of aqueous phase with the microcapsules. The aqueous phase was hidden to facilitate the analysis. A red line was drawn to mark the boundary of the porous medium.

Analyzing the images from the side of the fine spheres, it is possible to conclude that the microcapsules did not changed much the arrangement of oil ganglia. The microcapsules could penetrate a lot in the porous medium, but they were concentrated in the deeper layers, close to the coarse-fine spheres interface. It is important to mention that the microcapsules seen near the wall, immediately after the large oil ganglion at the inlet were not into the porous medium.

\section{3}

\section{Image processing quantification}

Quantitative results were obtained for one experiment performed with the homogeneous porous medium, using the device " $\mathrm{d}$ ", that has a porosity of $40.4 \%$ and permeability of $193 \mathrm{D}$. The images obtained came from the middle region of the porous medium (as shown in Fig. 4.1). This experiment was not reported in the previous sections, and was run according to a different experimental procedure. These were the steps taken:

- Device saturation with the aqueous phase; 
- Device saturation with the oil phase at $700 \mu l / h$;

- Aqueous phase injection at $25 \mu l / h(\approx 10 \mathrm{PV})$;

- Aqueous phase injection at $500 \mu l / h(\approx 10 \mathrm{PV})$;

- Capsule injection at $500 \mu l / h$;

- Aqueous phase injection at $500 \mu l / h(\approx 10 \mathrm{PV})$.

At the end of each one of these steps, after the system reached steady state, the image of the device was acquired and the oil saturation was measured. The data acquired during each phase of the experiment passed through the image processing and then it was possible to execute a qualitative analysis, as explained in Experimental Procedure chapter. It was used, as mentioned there, the Otsu automatic segmentation method for phases distinction.

Table 4.1 shows the oil saturation measured through image analysis after each step of the experimental procedure. It is important to mention that the method quantifies the amount of space occupied by each phase and by the spheres. Thus, the two first columns of Table 4.1 added together should sum up to a value close to the porosity of the device, $40.4 \%$.

This condition is roughly observed for most of the steps of the experiment, except for the aqueous phase saturation. The reason is probably related to the stabilization of the $\mathrm{CO}_{2}$ initially present in the device that may change the refractive index of it. However, at this step no oil was present, so the result is not very relevant.

In the last column of Table 4.1 are presented normalized values for the amount of oil phase, the $S_{o r}$. It is interesting to notice that the amount of oil decreased with the increase in aqueous phase flow rate and after the microcapsules were injected. However, making any conclusion from this behavior could be wrong. That because oil ganglia from the region upstream from the investigated region could be entering into it, and in this case the $S_{\text {or }}$ could even increase after the steps, without invalidating any conclusion about microcapsules recovery efficiency.

So, these results are mentioned in this work just to show that is possible to obtain quantitative data from the 3D images acquired. Nevertheless, to reach a firm conclusion on microcapsules sweep efficiency it is needed to investigate an image along the whole porous medium. This would require much more processing time and computational memory and was not tried in the scope of this work. 
Table 4.1: Quantification of the oil phase, aqueous phase and the residual oil saturation $\left(S_{o r}\right)$ after each phase saturation and after the aqueous phase injection and aqueous phase with capsule suspension injection. Experiment performed using device " $\mathrm{d}$ ".

\begin{tabular}{|c|c|c|c|}
\hline & $\begin{array}{c}\text { Oil Phase } \\
\text { (\% of Total Volume) }\end{array}$ & $\begin{array}{l}\text { Aqueous Phase } \\
\text { (\% of Total Volume) }\end{array}$ & $S_{o r}(\%)$ \\
\hline $\begin{array}{l}\text { Aqueous } \\
\text { Saturation }\end{array}$ & 0 & 29.6 & 0 \\
\hline $\begin{array}{c}\text { Oil } \\
\text { Saturation }\end{array}$ & 30.3 & 5.8 & 84.0 \\
\hline $\begin{array}{l}\text { Aqueous } \\
\text { Injection } \\
(25 \mu l / h)\end{array}$ & 23.2 & 21.7 & 51.6 \\
\hline $\begin{array}{l}\text { Aqueous } \\
\text { Injection } \\
(500 \mu / / h)\end{array}$ & 13.4 & 27.4 & 32.9 \\
\hline $\begin{array}{c}\text { Capsules } \\
\text { Injection } \\
\text { (shortly after the start) }\end{array}$ & 9.7 & 28.1 & 25.7 \\
\hline $\begin{array}{l}\text { Capsules } \\
\text { Injection } \\
\text { (after 10 PV) }\end{array}$ & 9.5 & 29.0 & 24.1 \\
\hline
\end{tabular}

In any case, the results from Table 4.1 show unequivocally that microcapsules mobilized oil ganglia out of the region investigated, since the $S_{\text {or }}$ continuously decreases after each step. This indirectly proves, without a precise quantification, its efficiency on oil ganglia sweep.

Another interesting aspect that can be observed, is that the results suggest that the microcapsules acts almost immediately after the beginning of the injection, and keeping injecting larger volumes of aqueous phase did not improved the oil sweep.

Figure 4.42 shows perspective views of the device " $\mathrm{d}$ " before and after the microcapsules injection. As can be seen, the amount of oil inside the porous medium after the microcapsules injection appears to be smaller than before the injection. 

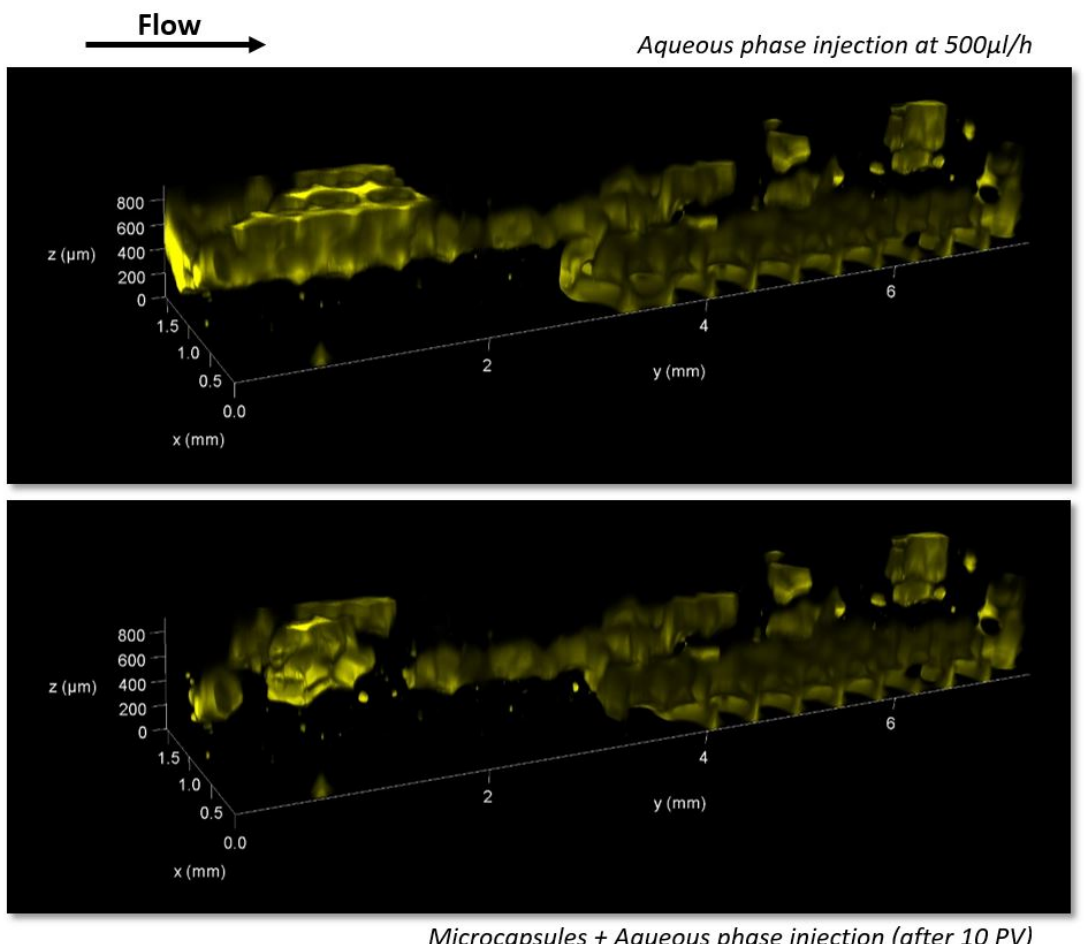

Microcapsules + Aqueous phase injection (after 10 PV)

Figure 4.42: Perspective view of device "d" before and after the microcapsules injection.

Regarding the quantification method, a test using another automatic segmentation algorithm, the Yen method, was also performed. The result is shown in Fig. 4.43, where the blue bars are the values of $S_{\text {or }}$ obtained using the Otsu method and the orange bars were obtained using the Yen method.

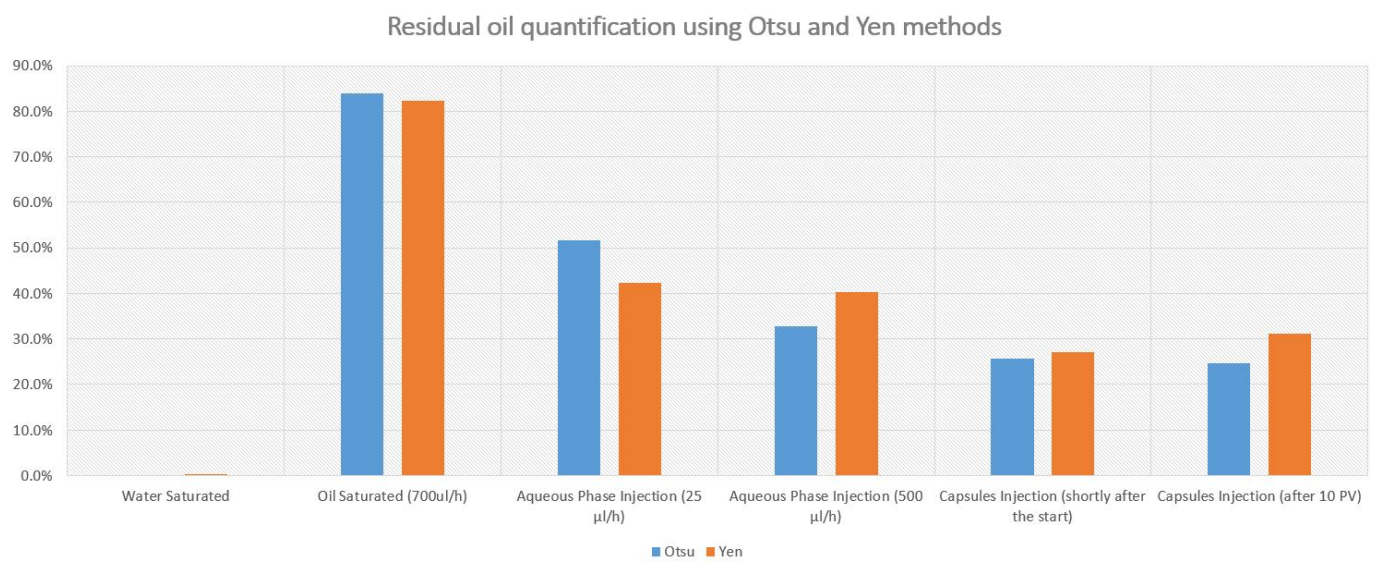

Figure 4.43: Comparison graph between the $S_{\text {or }}$ values obtained using the Otsu automatic segmentation method (blue) and the Yen method (orange) for images from each step of the experiment with device " $d "$.

It is possible to conclude by analyzing Fig. 4.43, that the Yen automatic segmentation method presented a similar behavior for the oil recovery during 
the experiment. The main difference between these methods was the observation of a small increase in oil residual saturation after $10 \mathrm{PV}$ of aqueous phase and capsules were injected. Which as mentioned before can even be a physical possibility.

In general, due to the aspects discussed, the results obtained shall be analyzed with a certain reservation. Nevertheless, they point that future works may stress out the limitations faced by this one and obtain a more conclusive quantitative analysis of similar phenomenon.

\section{4}

\section{Dynamic visualization}

Working together with other researchers from LMMP, a preliminary test was performed in order to dynamically visualize the movement of the microcapsules during the injection phase. For this, it was selected a single visualization region inside the porous medium and, in order to accelerate the acquisition, some microscope settings were modified. In this experiment, no oil was injected into the porous medium, only the aqueous phase and the microcapsules.

The microscope setup is summarized in Fig. 4.44. It acquired 48 times a complete 3D image in sequence. Each 3D image took around 16 seconds to be acquired and the total acquisition duration was 13 minutes and 11 seconds. Each 3D image obtained can be considered as a frame of the dynamic visualization (video). Obviously, a short delay could be observed between two frames.

Each 3D image was composed by a stack of 82 planes with 512 x 512 pixels, mapping a $366.43 \times 366.43 \mu \mathrm{m}$ region (resolution of $0.717 \mu \mathrm{m}$ per pixel). The resolution in the $z$ direction was the distance between each $2 \mathrm{D}$ image in the stack, that was $2.411 \mu \mathrm{m}$. The total region visualized was $366.43 \times 366.43$ x $195.28 \mu \mathrm{m}$. Important to notice that in this experiment, differently from the others, the microcapsules were colored yellow. The aqueous phase remained represented by blue. 


\begin{tabular}{|c|c|c|c|c|}
\hline \multicolumn{5}{|l|}{ Summary } \\
\hline Image & \multicolumn{4}{|l|}{ Series013 } \\
\hline Size & \multicolumn{4}{|l|}{$1.9 \mathrm{~GB}$} \\
\hline Dimensions & \multicolumn{4}{|c|}{$512 \times 512 \times 82,2$ Channels, 8 bits, t: 48} \\
\hline File Location & \multicolumn{4}{|c|}{ C:YUsers\TCS-User\Desktop\Project001.lif } \\
\hline Acquition Start & \multicolumn{4}{|l|}{ November 23, 2017 6:01 PM } \\
\hline Acquition Duration & \multicolumn{4}{|l|}{$13 \mathrm{~m} \mathrm{11s}$} \\
\hline \multicolumn{5}{|l|}{4 Dimensions } \\
\hline \multicolumn{2}{|r|}{ Logical Size } & \multicolumn{2}{|l|}{ Physical Length } & Voxel Size \\
\hline \multicolumn{2}{|r|}{512} & \multicolumn{2}{|l|}{$366.43 \mu \mathrm{m}$} & $0.717 \mu \mathrm{m}$ \\
\hline \multicolumn{2}{|r|}{512} & \multicolumn{2}{|l|}{$366.43 \mu \mathrm{m}$} & $0.717 \mu \mathrm{m}$ \\
\hline \multicolumn{2}{|l|}{ z } & \multicolumn{2}{|l|}{$195.28 \mu \mathrm{m}$} & $2.411 \mu \mathrm{m}$ \\
\hline \multicolumn{2}{|l|}{ Time } & \multicolumn{2}{|l|}{$13 \mathrm{~m} \mathrm{4s}$} & \\
\hline \multicolumn{5}{|l|}{ Time Stamps } \\
\hline \multicolumn{5}{|l|}{ Detection } \\
\hline Detector & Detection Range & Color & \multicolumn{2}{|l|}{ Gain } \\
\hline PMT 1 & $500 \mathrm{~nm}-600 \mathrm{~nm}$ & Yellow & \multicolumn{2}{|l|}{$705 \mathrm{~V}$} \\
\hline HyD 2 & $665 \mathrm{~nm}-700 \mathrm{~nm}$ & Cyan & \multicolumn{2}{|c|}{$10 \mathrm{~V}$ Standard Mode } \\
\hline \multicolumn{5}{|l|}{ Excitation } \\
\hline Laser Lines & \multicolumn{4}{|l|}{$\begin{array}{ll}488 \mathrm{~nm} & 6.0 \% \\
638 \mathrm{~nm} & 8.0 \%\end{array}$} \\
\hline \multicolumn{5}{|l|}{ Confocal Settings } \\
\hline System & \multicolumn{4}{|l|}{ TCS SP8 DMI8-CS } \\
\hline Scan Mode & \multicolumn{4}{|l|}{ xyzt } \\
\hline Scan Speed & \multicolumn{4}{|l|}{$12000 \mathrm{~Hz}$} \\
\hline Format & 8 bits & & & \\
\hline Pinhole & 1.0 airy units at $580 \mathrm{~nm}$ & & & \\
\hline Objective Lens & HC PL APO CS $10 x / 0.40$ DRY & A: 0.40 , Refractive & & \\
\hline Zoom & 3.17 & & & \\
\hline Bidirectional & Yes & & & \\
\hline Accumulation & Line Average 4 & & & \\
\hline
\end{tabular}

Figure 4.44: Microscope settings for the dynamic visualization of capsules blocking pores. Aqueous phase is represented by blue and the microcapsules by yellow.

Using a different objective lens from the rest of the experiments, HC PL APO CS 10x/0.40 DRY, a zoom factor of 3.17 was selected and a line average value of 4 .

The resulting video obtained is available at the internet at https://youtu.be/vnJVlEDhgOA. Six frames of the video developed are presented in Fig. 4.45. By the order, the figure presents the 2nd, 7th, 11th, 24th, 30 th and 44th frames of the movie. Thus, there is a gap of 80 seconds between the first and the second, 64 seconds between the second and the third, 368 seconds between the third and the forth, 96 seconds between the forth and the fifth and 224 seconds between the fifth and the sixth. In the images the flow is from the bottom right corner of the image to the upper left corner. 
It is important to notice that the acquisition time was short enough to well observe the capsules movement without incomprehensible jumps between the image sequence.

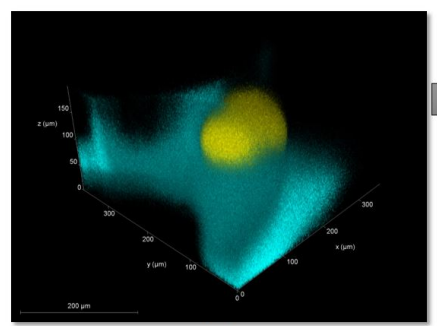

(1)

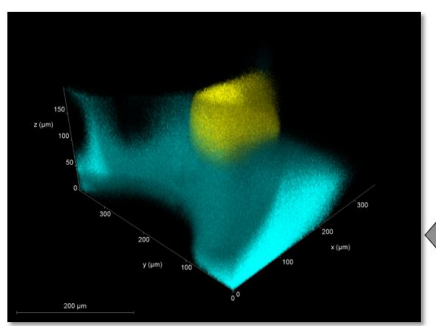

(6)

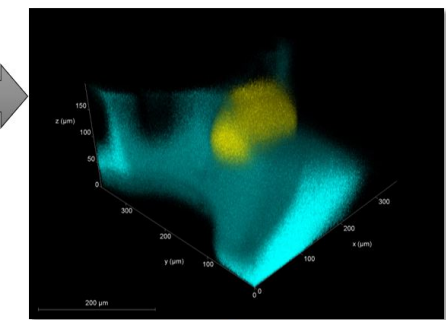

(2)

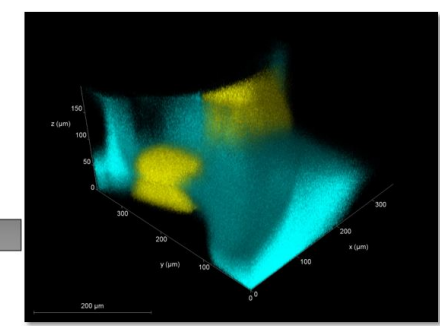

(5)

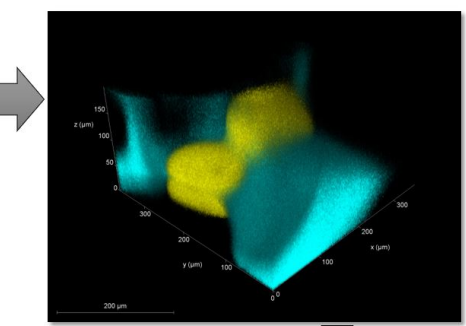

(3)

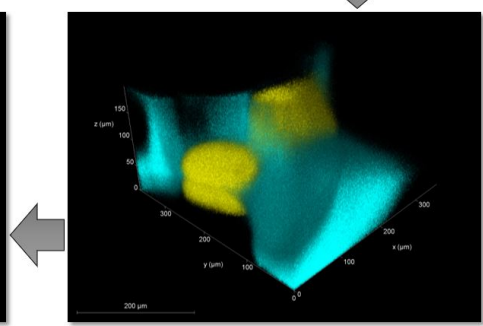

(4)

Figure 4.45: Different moments of the dynamic visualization.

In the images it is possible to first see a microcapsules trapped in the space between glass spheres. Then, it seems to deform under the flow, and eventually a second microcapsules comes to the region. The two microcapsules keep suffering deformation to the point that one of them escapes from that region.

No major conclusion is aimed by this dynamic acquisition. It is presented here just to show that it is possible to produce such kind of information, that can be stressed out in future works under a specifically designed experiment.

\section{5}

\section{Capillary devices with thinner walls}

Finally, a specific problem was detected in the visualization. The border of the 2D images were darker than the middle region. This results in difficulties in the numerical phase segmentation process. After gaining more knowledge on the equipment and its limitations and possibilities, a strategy to improve the quality of the image was proposed to remediate this problem.

A square tube capillary with thinner walls was purchased and tested. It was found that it imposed some difficulties at the porous device fabrication. On the other hand, after overcoming these difficulties by changing the setup of the sintering process, it was possible to obtain images with better visualization quality close to the borders. 
Figure 4.46 shows a comparison of two images of devices with different wall thickness, both composed by $425 \mu \mathrm{m}$ glass spheres. It is clear that using a square capillary with thinner walls lead to images with better quality at the border.

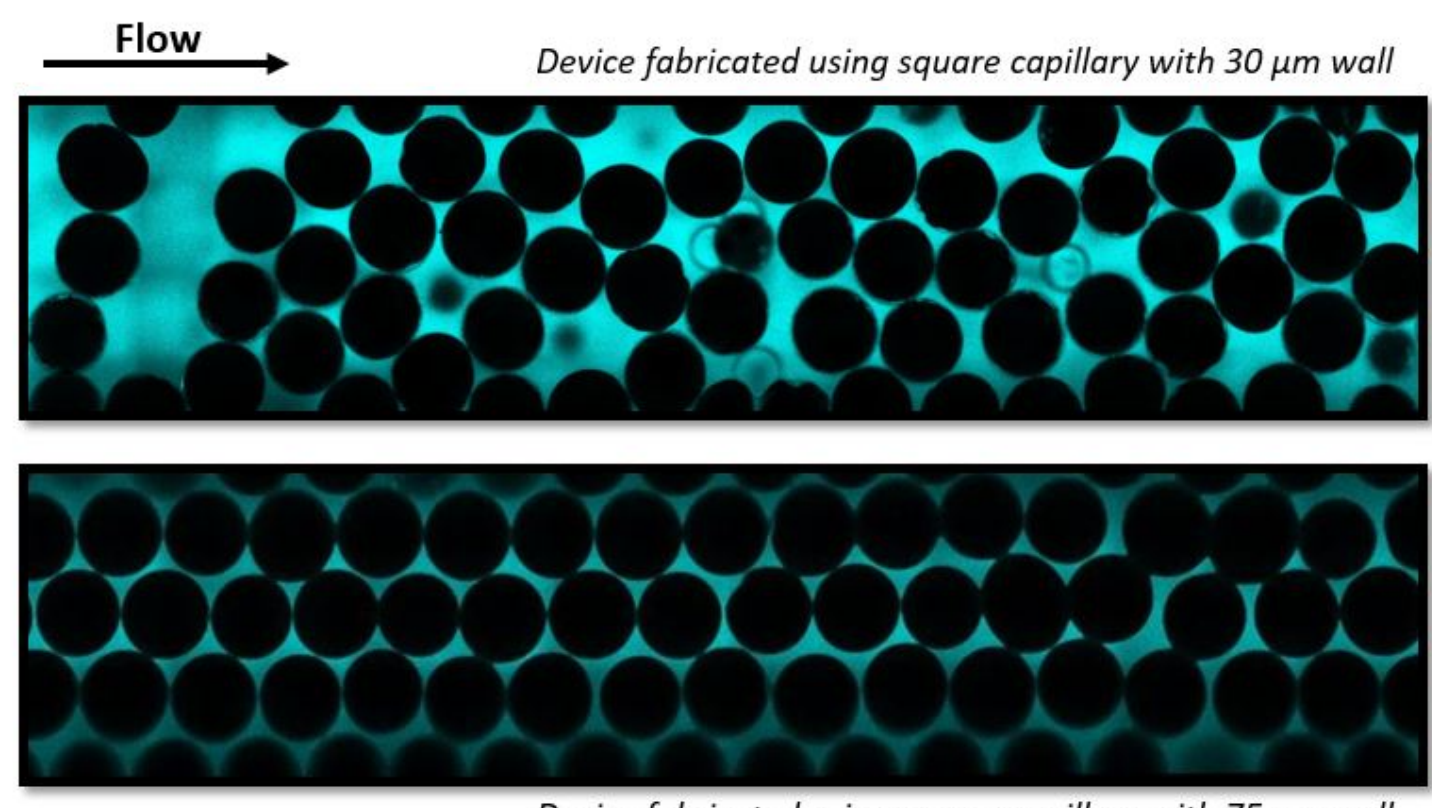

Device fabricated using square capillary with $75 \mu \mathrm{m}$ wall

Figure 4.46: Comparison between images obtained after aqueous phase saturation using devices with two different wall thickness.

Since this improvement was achieved only at the final phase of this work, it was not implemented for the data presented here. However, this improvement is mentioned as a reference for future research in the area. 


\section{Conclusions}

This research developed a protocol to perform experiments using CLSM techniques to visualize the effects of the injection of gellan gum based microcapsules to enhance oil recovery. While developing this protocol, some improvements for the confocal technique and for the experimental setup were proposed and successfully implemented. A large amount of qualitative information and even some quantitative information as well was obtained, suggesting that microcapsules are an effective strategy for mobilizing oil ganglia in porous media.

\section{1}

\section{Remarks on the experimental setup}

\subsection{1}

\section{Remarks on the technique for visualization with the CLSM}

The simultaneous visualization of the two phases and the microcapsules, distinctively, was possible due the use of three different fluorescent pigments, one for each phase.

It was used a tool of the Leica software that performs the acquisition to increase the depth of visualization. With this tool, the laser intensity and numerical gain could be linearly compensated through the focal plane position. Then, it was possible to get information of phase distribution up to $1 \mathrm{~mm}$ depth into the device.

A method for the measurement of the refractive indices for glass beads was verified and it was found that it can be used as a preliminary screen to limit the ranges to be used in the immersion technique.

A thinner wall type of square tube capillary was purchased and tested during the development of this work. It imposed more difficulties to the porous device fabrication but, overcoming these difficulties, it was possible to obtain clearer images with the CLSM technique. The use of thinner walls capillaries lead to images without the shadows near the capillary border that appeared due the wall curvature. 


\subsection{2 \\ Remarks on the experimental setup for microcapsules injection in porous media}

A device holder was designed and used in this work allowing to reproduce each step, even on different experiments, at the same device position. Using this holder, it was needed to make an adaption in order to visualize the entrance of the porous medium but it worked as well.

A new device construction procedure was developed, enabling to axially rotate the porous device and to ensure a more even distribution of the glass spheres at the entrance of the device. Also, the changes made even possible to reuse the device in case of the injection tubes were damaged.

Two types of porous media were produced in this work. The easier to fabricate was the homogeneous porous medium, with one sized glass spheres. The stratified device was composed by two separated layers of different size glass spheres.

Many soft gellan gum microcapsules sizes and with different shell thickness were produced and tested. The capsules chosen for most experiments in this work were the ones with smaller diameter and shell thickness. They were a little bit smaller than the theoretical porous channels dimension and this made easier for them to penetrate the porous medium. It is important to remind that only when a number of capsules penetrate in the porous channels it was possible to observe changes in the flow structure.

It was found that the best way to guarantee that the microcapsules reach the porous medium was to position them directly into the injection tube, avoiding to pass them through $\mathrm{T}$ connections or syringes.

\section{2}

\section{Remarks on the results obtained}

The oil phase used in this work was different from previous investigations, in order to provide a higher viscosity ratio between phases. With this higher viscosity ratio, it was possible to obtain conditions that increased the chances for the capsules to penetrate in the porous medium.

Also, with the purpose of making easier the entrance of the microcapsules in the porous medium, a gradual increase of the aqueous phase injection was prescribed. That allowed to achieve higher pressures without recovering all the oil inside the porous medium before the capsule injection.

By implementing these improvements it was possible to generate different porous media flow situations and to acquire a large number of visual information about these situations, in the form of 2D and 3D images. These images 
allowed to make some inferences on the flow and, specially, on the effects of the injection of microcapsules into homogeneous and stratified porous media.

In the experiments, it was possible to obtain a detailed visualization of irreducible aqueous phase after the oil saturation step.

Analyzing the images obtained at the experiment, the oil ganglia distribution after the aqueous phase injection and after the aqueous phase with microcapsules suspension injection were clearly observed.

In some experiments, it was possible to observe a large amount of oil trapped at the entrance of the porous medium after the aqueous phase injection. As the oil was the non wetting phase in these experiments, the aqueous phase flow was not enough to force this oil to further penetrate into the porous medium. After the microcapsules injection many changes in the flow pattern occurs, mainly at the entrance of the porous medium, as it was the region that accumulated a large amount of capsules. In some experiments it was possible to see that the presence of the microcapsules forced the oil originally trapped at the entrance downstream.

One of the challenges for this work was to develop conditions in which the microcapsules could penetrate the porous medium without provoking the full oil recovery by the previous aqueous phase injection. This goal was successfully achieved, and, by that, it was possible to observe how a considerable amount of capsules were needed blocking the near region of a ganglion in order to mobilize it. Many experimental images showing the oil displacement after the microcapsules injection were obtained. Some of them lead to the conclusion that small quantities of microcapsules were not enough to enhance oil mobilization. It was also possible to observe that some of the oil ganglia that were not fully mobilized by the microcapsules injection suffered deformations in their shape after the flow changes caused by the microcapsules blockage.

Besides the images that clearly show the mobilization of the oil ganglia due the action of the microcapsules, another evidence of their effectiveness was the observation, during the experiments, of amounts of oil passing through the exit tube. This was observed in many experiments and it is important to remember that no oil passed through this tube after reaching the steady state of aqueous phase injection.

A technique to quantify the volume occupied by each phase and by the microcapsules was developed and with that it was possible to obtain quantitative data for one of the 3D images acquired in this work. The results obtained are physically consistent and point to the fact that microcapsules injection can mobilize oil trapped after aqueous phase injection. 
Finally, an experiment focusing on a small region of the porous medium with faster acquisition was performed in order to visualize the dynamical behavior of the microcapsules inside the porous medium. The dynamical experiment generate a video in which the frames are the sequence of 3D images.

\section{3}

\section{Future works}

Due to time limitations, some experiments, improvements and analysis were not fully developed in the scope of this work and remain as suggestions for future works:

- upload and publish the data obtained in this work in the https://www.digitalrocksportal.org/ website;

- perform longer experiments with the visualization of a larger region, maybe the full device. To this, more computing resources may be needed;

- execute the quantitative analysis procedure in images obtained from other experiments;

- analyze the volume and the distribution of the oil ganglia before and after the microcapsules injection;

- measure, during the experiment, the pressure drop and the expected variation of the device permeability after the microcapsules injection;

- perform experiments with higher control on the quantity of microcapsules injected and test the injection of larger volumes of microcapsules;

- test and analyze the effect of a gradual injection of microcapsules;

- modify the experiment in order to quantify the amount of microcapsules needed to mobilize oil ganglia inside the porous medium;

- analyze the microcapsules distribution inside the device after injections at different flow rates;

- produce and test smaller microcapsules or emulsions;

- merge the 3D images from the opposite sides of the device to reconstruct the whole porous medium in a single stack;

- test the implementation of more advanced tools, such as deconvolution, in the image process procedure. 


\section{4}

\section{Final remarks}

A perspective for the future is that the microcapsules, or another type of controlled emulsion, injection could be applied in the oil industry as a method to enhance oil recovery. In this work, the oil recovery application using microcapsules was studied using a simplified experimental model and a technique to develop experiments and visualization analysis was presented. However, it is important to notice that, in real reservoir conditions, much smaller capsules would be needed to achieve a similar result as the porous channels could be tens to hundreds times smaller than the channels of the porous media used in this work.

Despite all the difficulties, the experimental procedure and the results presented in this work could be considered as an advance to understand the phenomenon of suspensions injection through a porous media. 


\section{Bibliography}

[1] ENGELKE, B. S.. Determinação das curvas de permeabilidade relativa no escoamento de emulsões e Óleo. Master's thesis, PUC-Rio, 2012.

[2] LENORMAND, R.. Liquids in porous media. J. Phys.: Condens. Matter, 2:79-88, 1990.

[3] CHUOKE, R. L.; VAN MEURS, P. ; VAN DER POEL, C.. The instability of slow, immiscible, viscous liquid-liquid displacements in permeable media. Petroleum Transactions, AIME, 216:188-194, 1959.

[4] KANTZAS, A.; BRYAN, J. B. ; TAHERI, S.. Fundamentals of Fluid Flow in Porous Media. PERM Inc., 2019.

[5] DATTA, S. S.; RAMAKRISHNAN, T. S. ; WEITZ, D. A.. Mobilization of a trapped non-wetting fluid from a three-dimensional porous medium. Physics of Fluids, 26(2):022002, feb 2014.

[6] PAYATAKES, A. C.. Dynamics of Oil Ganglia During Immiscible Displacement in Water-Wet Porous Media. Annual Review of Fluid Mechanics, 14:365-393, 1982.

[7] HINKLEY, R.. Oil Ganglion Motion. PhD thesis, University of Houston, 1981.

[8] OLAJIRE, A. A.. Review of ASP EOR (alkaline surfactant polymer enhanced oil recovery) technology in the petroleum industry: Prospects and challenges. Energy, 77:963-982, dec 2014.

[9] MCAULIFFE, C. D.. Crude-Oil-Water Emulsions to Improve Fluid Flow in an Oil Reservoir. Journal of Petroleum Technology, 25:721 726, 1973.

[10] COBOS, S.; CARVALHO, M. ; ALVARADO, V.. Flow of oil-water emulsions through a constricted capillary. International Journal of Multiphase Flow, 35(6):507-515, jun 2009. 
[11] MCAULIFFE, C. D.. Crude-Oil-Water Emulsions to Improve Fluid Flow in an Oil Reservoir. Journal of Petroleum Technology, (06):721726, 1973.

[12] DO NASCIMENTO, D. F.; AVENDAÑO, J. A.; MEHL, A.; MOURA, M. J. B.; CARVALHO, M. S. ; DUNCANSON, W. J.. Flow of Tunable Elastic Microcapsules through Constrictions. Scientific Reports, 7(1):11898, $\operatorname{dec} 2017$.

[13] VIMIEIRO JÚNIOR, J. R.. Visualização Tridimensional do Deslocamento de Óleo por Suspensões de Microcápsulas Flexíveis em Meios Porosos. Master's thesis, PUC-Rio, 2017.

[14] MICHELON, M.; LEOPÉRCIO, B. C. ; CARVALHO, M. S.. Microfluidic production of aqueous suspensions of gellan-based microcapsules containing hydrophobic compounds. 2019.

[15] SEMWOGERERE, D.; WEEKS, E. R.. Confocal microscopy. In: ENCYCLOPEDIA OF BIOMATERIALS AND BIOMEDICAL ENGINEERING, BIOMATERIALS, BIOMEDICAL ENGINEERING. INFORMA HEALTHCARE, 2005.

[16] CLAXTON, N. S.; FELLERS, T. J. ; DAVIDSON, M. W.. Laser Scanning Confocal Microscopy. Technical report.

[17] BORLINGHAUS, R. T.. Confocal Microscopy, may 2011.

[18] HOVIS, D. B.; HEUER, A. H.. The use of laser scanning confocal microscopy (LSCM) in materials science. Journal of Microscopy, 240(3):173-180, dec 2010.

[19] KRUMMEL, A. T.; DATTA, S. S.; MÜNSTER, S. ; WEITZ, D. A.. Visualizing multiphase flow and trapped fluid configurations in a model three-dimensional porous medium. AIChE Journal, 59(3):1022-1029, mar 2013.

[20] DATTA, S. S.. Getting Out of a Tight Spot: Physics of Flow Through Porous Materials. PhD thesis, 2013.

[21] YUE, L.-Y.; WANG, P. ; HUANG, Y.-X.. Easy method to determine refractive indices of microspheres and in micro-regions of inhomogeneous media. Biosensors and Bioelectronics, 30:216-222, 2011.

[22] SAHIMI, M.. Flow and transport in porous media and fractured rock: from classical methods to modern approaches. 2011. 
[23] CARMAN, P.. Fluid flow through granular beds. Transactions of the Institution of Chemical Engineers, 15:S32-S48, 1937.

[24] YORK, R.. Do alternative energy sources displace fossil fuels? Nature Climate Change, 2(6):441-443, jun 2012.

[25] DE OLIVEIRA CRUZ, G. B.. Visualization in micromodels of oil displacement by o/w emulsions. Master's thesis, PUC-Rio, 2018.

[26] HEIJS, A. W.; RITSEMA, C. J. ; DEKKER, L. W.. Three-dimensional visualization of preferential flow patterns in two soils. Geoderma, 70:101 - 116, 1996.

[27] DULIU, O. G.. Computer axial tomography in geosciences: an overview. Earth-Science Reviews, 48:265 - 281, 1999.

[28] CÍSLEROVÁ, M.; VOTRUBOVÁ, J.. CT derived porosity distribution and flow domains. Journal of Hydrology, 267:186 - 200, 2002.

[29] ESPINAL, L.. Porosity and Its Measurement. In: CHARACTERIZATION OF MATERIALS, p. 1-10. John Wiley \& Sons, Inc., Hoboken, NJ, USA, oct 2012.

[30] GREVERS, M. C. J.; DE JONG, E. ; ARNAUD, R. J. S.. The characterization of soil macroporosity with CT scanning. Canadian Journal of Soil Science, 1989.

[31] PYRAK-NOLTE, L. J.; MONTEMAGNO, C. D. ; NOLTE, D. D.. Volumetric imaging of aperture distributions in connected fracture networks. Geophysical Research Letters, 24(18):2343-2346, sep 1997.

[32] SINGH, K.; BIJELJIC, B. ; BLUNT, M. J.. Imaging of oil layers, curvature and contact angle in a mixed-wet and a water-wet carbonate rock. Water Resources Research, 52(3):1716-1728, mar 2016.

[33] DULIU, O. G.. Computer axial tomography in geosciences: an overview. Earth-Science Reviews, 48(4):265-281, dec 1999.

[34] BLUNT, M. J.. Multiphase flow in permeable media: A pore-scale perspective. 2017.

[35] ALAGORNI, A. H.; YAACOB, Z. B. ; NOUR, A. H.. An Overview of Oil Production Stages: Enhanced Oil Recovery Techniques and Nitrogen Injection. International Journal of Environmental Science and Development, 6(9), 2015. 
[36] HOMSY, G.. Viscous Fingering In Porous Media. Annual Review of Fluid Mechanics, 19(1):271-311, 1987.

[37] THOMAS, S.; FAROUQ ALI, S. M.. Flow of emulsions in porous media, and potential for enhanced oil recovery. Journal of Petroleum Science and Engineering, 3(1-2):121-136, 1989.

[38] LEE, M. H.; HRIBAR, K. C.; BRUGAROLAS, T.; KAMAT, N. P.; BURDICK, J. A. ; LEE, D.. Harnessing Interfacial Phenomena to Program the Release Properties of Hollow Microcapsules. Advanced Functional Materials, 22(1):131-138, jan 2012.

[39] LEE, H.; CHOI, C.-H.; ABBASPOURRAD, A.; WESNER, C.; CAGGIONI, M.; ZHU, T. ; WEITZ, D. A.. Encapsulation and Enhanced Retention of Fragrance in Polymer Microcapsules. ACS Applied Materials \& Interfaces, 8(6):4007-4013, feb 2016.

[40] VERICELLA, J. J.; BAKER, S. E.; STOLAROFF, J. K.; DUOSS, E. B.; HARDIN, J. O.; LEWICKI, J.; GLOGOWSKI, E.; FLOYD, W. C.; VALDEZ, C. A.; SMITH, W. L.; SATCHER, J. H.; BOURCIER, W. L.; SPADACCINI, C. M.; LEWIS, J. A. ; AINES, R. D.. Encapsulated liquid sorbents for carbon dioxide capture. Nature Communications, 6(1):6124, may 2015.

[41] HEPLER, P. K.; GUNNING, B. E. S.. Confocal fluorescence microscopy of plant cells Review article. Most, 201(3):121-157, 1998.

[42] PAWLEY, J. B.. Handbook of biological confocal microscopy. Springer, 2006.

[43] DATTA, S. S.; CHIANG, H.; RAMAKRISHNAN, T. S. ; WEITZ, D. A.. Spatial fluctuations of fluid velocities in flow through a threedimensional porous medium. Physical Review Letters, 111(6), 2013.

[44] DATTA, S. S.; DUPIN, J. B. ; WEITZ, D. A.. Fluid breakup during simultaneous two-phase flow through a three-dimensional porous medium. Physics of Fluids, 26(6):062004, jun 2014.

[45] GONZALEZ, R. C.; WOODS, R. E.. Digital Image Processing, volumen 3. Pearson Education International, third edit edition, 1992.

[46] URUMOVIC, K.. The referential grain size and effective porosity in the Kozeny-Carman model. Hydrol. Earth Syst. Sci, 20:1669-1680, 2016. 
[47] DIAS, R. P.; FERNANDES, C. S.; MOTA, M.; TEIXEIRA, J. A. ; YELSHIN, A.. Permeability and effective thermal conductivity of bisized porous media. International Journal of Heat and Mass Transfer, 50(78):1295-1301, 2007.

[48] LEE, J.-S.. Digital image smoothing and the sigma filter. Computer Vision, Graphics, and Image Processing, 24(2):255-269, nov 1983. 


\section{A}

\section{Published paper}

RIBEIRO, R. C. O. S.; CARVALHO M. S, Visualization of microcapsules injection on oil recovery inside porous media using using confocal microscopy, submitted to COBEM 2019. 